La fabrication du droit de l'Union européenne dans le contexte du « Mieux légiférer »

sous la direction

de Nathalie Rubio

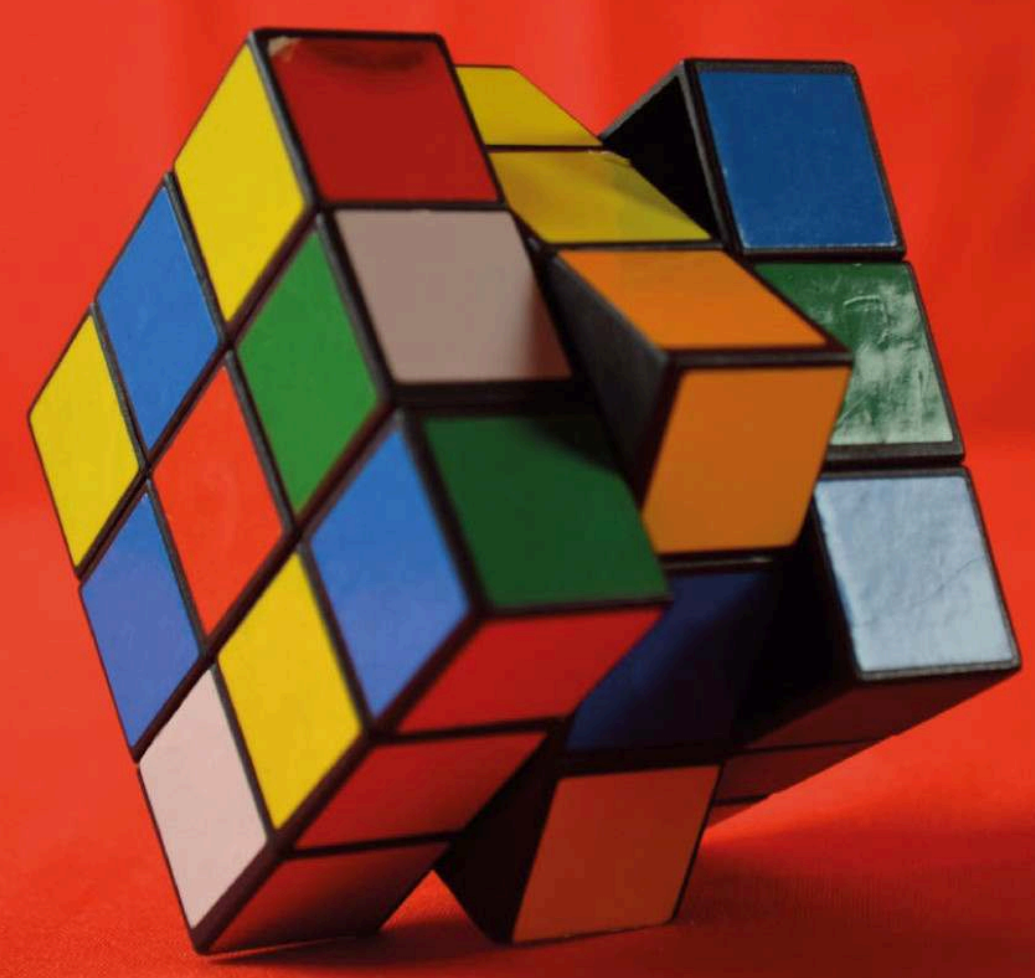




\section{La fabrication du droit de l'Union européenne dans le contexte du " Mieux légiférer »}

\section{Nathalie Rubio (dir.)}

DOI : 10.4000/books. dice.1308

Éditeur : DICE Éditions

Lieu d'édition : Aix-en-Provznce

Année d'édition : 2018

Date de mise en ligne : 11 février 2022

Collection : Confluence des droits

EAN électronique : 9791097578022

\section{OpenEdition}

\section{Books}

https://books.openedition.org

\section{Édition imprimée}

Nombre de pages : 220

\section{Référence électronique}

RUBIO, Nathalie (dir.). La fabrication du droit de l'Union européenne dans le contexte du « Mieux légiférer ». Nouvelle édition [en ligne]. Aix-en-Provence : DICE Éditions, 2018 (généré le 16 janvier 2023).

Disponible sur Internet : <http://books.openedition.org/dice/1308>. ISBN : 9791097578022 . DOI :

https://doi.org/10.4000/books.dice.1308.

Creative Commons - Attribution - Pas d'Utilisation Commerciale - Pas de Modification 4.0 International - CC BY-NC-ND 4.0 


\section{RÉSUMÉS}

Dans sa communication du 19 mai 2015, la Commission européenne se dit «déterminée à modifier à la fois ce que fait l'Union européenne (UE) et la façon dont elle le fait ». Elle y présente "de nouvelles mesures destinées à améliorer la réglementation pour obtenir de meilleurs résultats ». Elle souhaite ouvrir davantage le processus d'élaboration des politiques, ainsi que " mieux écouter et mieux interagir avec ceux qui mettent la législation de l'UE en œuvre et qui en bénéficient ». Il s'agit également de " jeter un regard neuf sur l'ensemble des domaines d'action pour déterminer ceux dans lesquels les mesures existantes demandent à être améliorées ». Cette dynamique a été confirmée dans une série d'autres communications et par l'Accord interinstitutionnel du 13 avril 2016. Pourtant, rien ne semble nouveau : cela prolonge un processus ouvert il y a près d'une quinzaine d'années avec le Livre Blanc sur la gouvernance européenne. Les contributions figurant dans cet ouvrage permettent de comprendre les textes relatifs au " Mieux légiférer » adoptés depuis 2015, d'opérer les comparaisons avec des expériences nationales et internationales, de poser un regard critique et d'imaginer des perspectives avec toujours à l'esprit la volonté de trouver les pistes pour tenter de dénouer le nœud gordien de la qualité, de la légitimité et de l'efficacité du droit de l'Union européenne.

\section{NATHALIE RUBIO (DIR.)}

Professeure de droit public, Chaire Jean Monnet, Aix Marseille Univ, Université de Toulon, Univ Pau \& Pays Adour, CNRS, DICE, CERIC, Aix-en-Provence, France 


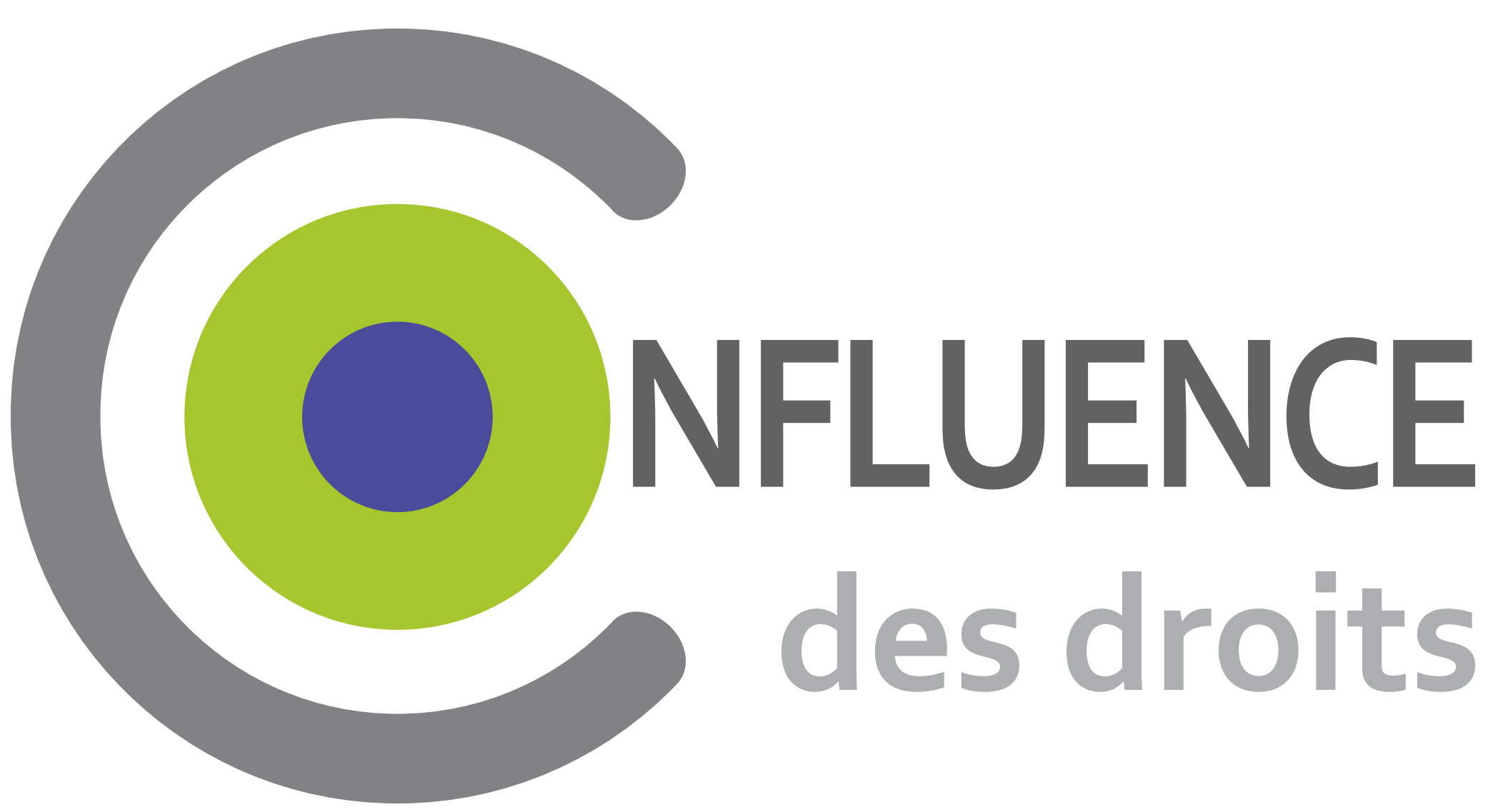

collection d'ouvrages numériques 



\section{NFLUENCE \\ des droits}




\section{Dans la même collection}

MALJEAN-DuboIs Sandrine (dir.), Circulations de normes et réseaux d'acteurs dans la gouvernance internationale de l'environnement, Confluence d es droits [ en ligne]. Aix-en-Provence : Droits International, Comparé et européen, 2017. ISBN : 979-10-97578-00-8

Fatin-Rouge Stefanini Marthe, SeVerino Caterina (dir.), Le contrôle de constitutionnalité des décisions de justice : Une nouvelle étape après la QPC ?, Confluence des droits [en ligne]. Aix-en-Provence : Droits International, Comparé et européen, 2017. ISBN : 979-10-97578-01-5

\section{http://dice.univ-amu.fr/fr/dice/dice/publications/confluence-droits}

ISBN : 979-10-97578-02-2

UMR Droits International, Comparé et Européen (DICE)

Espace René Cassin

3, avenue Robert Schuman

13628 Aix-en-Provence

dice-editions@univ-amu.fr

Références électroniques :

Rubio Nathalie (dir.), La fabrication du droit de l'Union européenne dans le contexte du «Mieux légiférer », Confluence des droits [en ligne]. Aix-en-Provence : Droits International, Comparé et européen, 2017.

Disponible sur Internet : http://dice.univ-amu.fr/fr/dice/dice/publications/confluence-droits ISBN : 979-10-97578-02-2. 


\title{
LA FABRICATION DU DROIT DE L'UNION EUROPÉENNE DANS LE CONTEXTE DU « MIEUX LÉGIFÉRER »
}

\author{
sous la direction de \\ Nathalie RuBIO
}

Avant-propos

Nathalie RuBıO

Préface

Rostane MEHDI 



\section{SOMMAIRE}

Avant-propos

Nathalie Rubio, Professeure de droit public, Chaire Jean Monnet, Aix Marseille Univ, Université de Toulon, Univ Pau \& Pays Adour, CNRS, DICE, CERIC, Aix-en-Provence, France

Préface

Rostane MeHdi, Professeur de droit public, Chaire Jean Monnet ad personam, Aix Marseille Univ, Université de Toulon, Univ Pau \& Pays Adour, CNRS, DICE, CERIC, Aix-en-Provence, France, Professeur au Collège d'Europe de Bruges, Directeur de SciencesPO Aix

Principaux sigles et abréviations .

Propos introductifs

Fabienne Péraldi Leneuf, Professeure à l'École de droit de la Sorbonne

\section{Partie I. Comprendre le «Mieux légiférer»}

\section{Chapitre 1. Le sens du «Mieux légiférer»}

Better regulation - Variations on a theme

Francis Snyder, C.V. Starr Professor of Law, EU Jean Monnet Chair ad personam and Director, Centre for Research on Transnational Law, Peking University School of Transnational Law, Peking University Shenzhen, China; Emeritus Professor, CERIC, Aix-Marseille University ; Visiting Professor, College of Europe, Bruges

La clarté rédactionnelle en pratique

Jennifer Gracie LL.B., Solicitor (non-practising) \& ancienne Membre du conseil de l'ordre de la Law Society de l'Angleterre et du Pays de Galles, Chercheur Associé de l'Institut des Hautes Études sur la Justice (Paris)

Chapitre 2. L'originalité du « Mieux légiférer » au regard des expériences internationales et nationales

Peut-on « Mieux légiférer » en droit international?

Romain Le B๕uf, Professeur de droit public, Aix Marseille Univ, Université de Toulon, Univ Pau \& Pays Adour, CNRS, DICE, CERIC, Aix-en-Provence, France

La conception du « Mieux légiférer » en droit constitutionnel .63

Marthe Fatin-Rouge Stefanini, Directrice de recherche, Aix Marseille Univ, Université de Toulon, Univ Pau \& Pays Adour, CNRS, DICE, GERJC-ILF, Aix-en-Provence, France 


\section{Partie II. Mettre en œuvre le «Mieux légiférer»}

\section{Chapitre 1. Approches transversales de la mise en œuvre du « Mieux légiférer »}

L'impact de la création des actes délégués sur les méthodes d'élaboration du droit de l'Union européenne - Le chemin vers l'enfer est pavé de bonnes intentions

Laetitia Guilloud-Colliat, Professeure de droit public, Faculté de droit de Grenoble, CESICE (EA 2420), Université Grenoble Alpes

Le « Mieux légiférer » et la limitation du recours aux actes législatifs en droit de l'Union... 95 Stéphane DE LA Rosa, Professeur de droit public, Université de Paris Est Créteil, MIL EA 7382

La Cour de justice de l'Union européenne et le «Mieux légiférer »-Recherche sur l'impact juridictionnel.

Nathalie Rubio, Professeure de droit public, Chaire Jean Monnet, Aix Marseille Univ, Université de Toulon, Univ Pau \& Pays Adour, CNRS, DICE, CERIC, Aix-en-Provence, France

L’impact du "Mieux légiférer » sur l'intégration du droit de l'Union européenne dans les États membres : le Mieux et le Bien transposer

Emmanuelle SAULnier-CASsia, Professeure de droit public, Université de Versailles-Saint-Quentin - Université Paris-Saclay

\section{Chapitre 2. Approches sectorielles de la mise en œuvre du « Mieux légiférer »}

«Mieux légiférer » - L'exemple des nouvelles technologies de l'information. 145 Jean-Claude Bonichot, Juge à la Cour de justice de l'Union européenne

Les principes du "Mieux légiférer " dans le domaine de l'environnement et de la santé : Quel bilan? Quelles perspectives?.

Estelle Brosset, Professeure de droit public, Chaire Jean Monnet, Aix Marseille Univ, Université de Toulon, Univ Pau \& Pays Adour, CNRS, DICE, CERIC, Aix-en-Provence, France

La politique sociale à l'aune du « Mieux légiférer »

Dominique Nazet-Allouche, Chercheure, Aix Marseille Univ, Université de Toulon, Univ Pau \& Pays Adour, CNRS, DICE, CERIC, Aix-en-Provence, France

Ambitions et insuffisances légistiques en matière de migration légale - L'application de la stratégie « Mieux légiférer » à la directive «Carte bleue».

Romain FoucART, Doctorant, Aix Marseille Univ, Université de Toulon, Univ Pau \& Pays Adour, CNRS, DICE, CERIC, Aix-en-Provence, France

Le « Mieux légiférer " au chevet de la politique commerciale commune de l'Union européenne - Potentiels et limites d'une recherche de légitimité par la procédure 


\title{
Avant-propos
}

\author{
Nathalie RuBio ${ }^{1}$
}

Les 6 et 7 octobre 2016 s'est tenu, à la Faculté de droit et de science politique de l'Université d'Aix-Marseille, un colloque international sur « La fabrication du droit de l'Union européenne dans le contexte du Mieux légiférer », organisé par le Centre d'Études et de Recherches Internationales et Communautaires (CERIC-UMR DICE 7318) et placé sous la direction scientifique des professeurs Rostane Mehdi, Fabienne Péraldi-Leneuf et Nathalie Rubio. Ce colloque a rassemblé près de 80 participants (universitaires français et étrangers, membres des institutions européennes, praticiens et étudiants).

Cette manifestation a reçu le soutien financier de l'Université d'Aix-Marseille, de SciencesPo Aix et de l'Université Paris Sud ainsi que celui des partenaires locaux qu'il convient également de remercier : la métropole Aix-Marseille Provence et le Département des Bouches du Rhône. Elle a également été soutenue par le Centre d'Excellence Jean Monnet «Europe au Sud » de l'Université d'Aix-Marseille.

Cette recherche s'inscrit en outre dans le prolongement de celle menée par le Réseau européen Jean Monnet «Solar », auquel le CERIC est partie, consacré à l'étude de la soft law européenne.

Ce colloque avait pour ambition d'analyser les textes relatifs au « Mieux légiférer » adoptés par les institutions européennes depuis 2015 au regard des documents et pratiques antérieurs, d'opérer les comparaisons avec des expériences nationales et internationales, de poser un regard critique et d'imaginer des perspectives avec toujours à l'esprit la volonté de trouver les pistes pour tenter de dénouer le nœud gordien de la qualité, de la légitimité et de l'efficacité du droit de l'Union européenne.

Les contributions et échanges ont été riches et ont justifié qu'une trace soit laissée par la publication d'une partie des communications. Le format électronique de la collection Confluence des droits permet une plus grande visibilité des résultats de ces réflexions et donne la possibilité, grâce à la souplesse de son format, d'envisager de revenir sur l'évolution de la mise en œuvre du « Mieux légiférer », ce qui devrait assurer un suivi de la recherche.

$\overline{1}$ Professeure de droit public, Chaire Jean Monnet, Aix Marseille Univ, Université de Toulon, Univ Pau \& Pays Adour, CNRS, DICE, CERIC, Aix-en-Provence, France. 
À l'image du cube, qui a été choisi comme thème du colloque et de la couverture de cet ouvrage, l'amélioration de la qualité de la législation européenne est un réel casse-tête. Toute la difficulté est de parvenir à dévoiler le mécanisme interne le plus adéquat pour répondre aux attentes de tous les acteurs intéressés (institutions, États, société civile) et, sous ces divers prismes, à déterminer celui le plus apte à aligner la même couleur sur chacune des faces. 


\title{
Préface
}

\author{
Rostane MEHDI ${ }^{1}$
}

«Le législateur n'est pas du côté de ceux qui ont l'art d'écrire pour écrire ou qui savent, pour notre plaisir, écrire n'importe quoi. Il est avec ceux qui ont quelque chose à dire et qui écrivent pour le dire. Avec eux, l'art d'écrire devient l'art de penser $»^{2}$. C'est en ces termes que G. Cornu évoquait l'art d'écrire la loi. Sans céder au tropisme d'un utilitarisme aveugle aux règles de l'esthétique, il n'y a guère de raisons de s'émouvoir que le «bien légiférer » revienne, en droit de l'Union aussi, à adopter des textes répondant au double impératif de qualité et d'efficacité.

L'idéal de la meilleure législation ${ }^{3}$ témoigne d'abord de la nécessité de dissiper le sentiment selon lequel le système juridique de l'Union serait, en quelque sorte par essence, promis à une irrémédiable autant qu'inexplicable confusion. Antienne incantatoire, cette complexité dégraderait l'attractivité de l'ordre juridique de l'Union. D'un point de vue substantiel, le brouillamini juridique serait à tous égard incompatible avec les exigences de la plus élémentaire sécurité juridique. Pour dire les choses sans détours, la loi peut être dense sans être fatalement absconse ou verbeuse.

Cette inflation est sans doute inévitable dans un ensemble qui assigne une fonction aussi centrale au droit. C'est par un volontarisme proliférant que l'Union impose son existence notamment aux États qui la composent. L'un des moyens d'éviter l'intempérance et le foisonnement juridique, est de procéder à un choix rigoureux des « véhicules normatifs » tout en s'astreignant à améliorer la qualité de la législation proprement dite. Outre la nécessité d'en soigner le processus de préparation, les institutions doivent, par une maîtrise rigoureuse des canons de la légistique, s'attacher à la qualité rédactionnelle des textes qu'elles adoptent ${ }^{4}$.

On ne doit cependant pas minimiser les contraintes qui pèsent sur l'acte de légiférer. Le Conseil, le Parlement et la Commission doivent en passer par de subtils arbitrages politiques. Or, la recherche d'un compromis s'inscrit dans un environnement répondant à des stimuli extrêmement variés. Rationalité politique et rationalité technique se trouveront souvent placées dans un rapport orthogonal.

\footnotetext{
1 Professeur de droit public, Chaire Jean Monnet ad personam, Aix Marseille Univ, Université de Toulon, Univ Pau \& Pays Adour, CNRS, DICE, CERIC, Aix-en-Provence, France, Professeur au Collège d'Europe de Bruges, Directeur de SciencesPO Aix.

G. Cornu, «L'art d'écrire la loi », in Le code civil, Pouvoirs, 2003/4, nº 107, p. 5.

F. Peraldi-Leneuf et S. De La Rosa (dir.), L’Union européenne et l'idéal de la meilleure législation, Iredies, Pedone, Cahiers européens, $\mathrm{n}^{\circ} 5,2013$.

4 Accord interinstitutionnel entre le Parlement européen, le Conseil de l’Union européenne et la Commission européenne du 13 avril 2016 , « Mieux légiférer », fOUE $\mathrm{n}^{\circ} \mathrm{L}$ 123, 12 mai 2016, p. 1.
} 
On butte sur les apories d'un système qui n'a cessé de dupliquer les procédures de contrôle et d'évaluation au risque d'altérer paradoxalement la qualité du droit. En effet, la multiplication des facteurs à prendre en compte dans le cadre par exemple des études d'impact est telle qu'elle conduit à brouiller l'intelligibilité des règles. Par ailleurs, le droit de l'Union s'inscrit souvent dans un registre technique. Aussi, peut-il s'engluer dans un jargon dont le législateur peine à faire son miel ${ }^{5}$. Il est vrai que les énoncés juridiques sont par nature polysémiques ; leur imprécision apparaissant même à la fois comme l'une de leurs « propriétés constitutives » et une condition de fonctionnement de la dogmatique juridique ${ }^{6}$. La qualité du droit ne serait finalement qu'un mythe renvoyant à des temps où la loi était simple, claire et concise, à un « âge d'or » auquel il suffirait revenir... Pour reprendre les mots du Doyen Carbonnier «l'inflation se grossit d'enflure. Sous cette dégradation de la forme se devine quelque chose d'infiniment plus grave, un changement profond dans la nature de la loi » ${ }^{7}$.

Produire le droit dans une société où la complexité est croissante tient inévitablement de la gageure. La loi résulte d'une combinaison alchimique entre impartialité et proximité, manifestations du bien en politique autant que condition de l'efficacité des normes. Les pouvoirs publics se doivent d'inclure l'ensemble des parties prenantes à des processus participatifs offrant à chacun le loisir d'exprimer, de manière publique et transparente, son point de vue sur les enjeux d'un exercice normatif. La légitimité du système dépend alors de la capacité des institutions décisionnaires à caler leur action sur la particularité des situations. La bonne mesure ne sera pas la plus générale mais bien celle dont les destinataires auront la conviction qu'elle a été prise en pleine connaissance de leur singularité. La promotion d'une gouvernance up to date amène à privilégier le développement de modes de production normative éventuellement alternatifs. On voit ainsi se mettre en place un système de «polyarchie délibérative $»^{8}$ mêlant participation (se déclinant elle-même en diverses variations), délégations contrôlées à des opérateurs privés et maintien de modalités plus classiques d'exercice de la puissance publique.

Répondre le plus justement possible aux attentes sociales ne doit cependant pas se faire au risque de la ductilité du droit. En effet, la loi ne saurait se concevoir en démocratie comme un acte fixé intemporellement. Elle doit au contraire s'adapter continuellement aux évolutions sociales sous peine d'une démonétisation des principes en fondant l'autorité. Pourtant, l'image d'un législateur incarnation d'une rationalité idéelle s'abstrayant de réalités prosaïques et capable d'assurer en toutes circonstances la cohérence de l'ordre juridique est extravagante. Devant l'ampleur de la tâche qui lui est assignée, le système de production normative de l'Union balbutie, s'emballe sans jamais s'étouffer. C'est ce que les pages qui suivront, à l'heureuse initiative de N. Rubio, explorent avec le souci d'une louable exhaustivité.

\footnotetext{
F. Martucci, «Jargon économique, mutation normative et outils légistiques », in F. Peraldi-Leneuf et S. De La Rosa (dir.), L’Union européenne et l'idéal de la meilleure législation, op.cit., sp. p. 79 et s.

J. Chevallier, «Peut-on rationaliser la production du droit? », in F. Peraldi-Leneuf et S. De La Rosa (dir.), L’Union européenne et l'idéal de la meilleure législation, op. cit., p. 25.

J. CARbonnier, Droit et passion du droit sous la $V^{e}$ république, Forum, Flammarion, 1996, p. 109

P. Magnette, Le régime politique de l'Union européenne, $3^{\mathrm{e}}$ éd., Les presses de Sciences Po, 2009, p. 256.
} 


\section{Principaux sigles et abréviations}

AFDI

AJDA

Aff.

C.

CJCE

CJUE

CMLR

EJIL

JCL

$\mathrm{JCP}$

JOUE

LPA

OCDE

Op. cit.

$\mathrm{Pt}$

RCADI

RDUE

Rec.

RFSP

RGDIP

RJE

RTDE

Trib.

TFUE

TUE

$\mathrm{V}$.
Annuaire français de droit international

Actualité juridique de droit administratif

Affaire

Contre

Cour de justice des Communautés européennes

Cour de justice de l'Union européenne

Common market law review

European journal of international law

Juris-Classeur

Juris-Classeur périodique

Journal officiel de l'Union européenne

Les petites affiches

Organisation de coopération et de développement économique

Référence précitée

Point

Revue des cours de l'Académie de droit international de La Haye

Revue de droit de l'Union européenne

Recueil

Revue française de science politique

Revue générale de droit international public

Revue juridique de l'environnement

Revue trimestrielle de droit européen

Tribunal de l'Union européenne

Traité sur le fonctionnement de l'Union européenne

Traité sur l'Union européenne

Voir 



\title{
Propos introductifs
}

\author{
Fabienne PÉRALDI LENEUF ${ }^{1}$
}

Le thème de cet ouvrage et des deux journées d'études qui lui ont été consacrées en octobre 2016 à la Faculté de droit et de science politique d'Aix-en-Provence manifeste une préoccupation grandissante en Europe depuis plusieurs années déjà et plusieurs colloques et études lui ont été ainsi consacrés depuis le milieu des années $2000^{2}$. Dès lors, il ne serait pas illogique de se demander ce qui peut justifier de nouveaux travaux aujourd'hui. Que pourrait-on dire qui n'ait déjà été traité ?

Justement, l'intérêt du sujet relatif à l'action européenne « Mieux légiférer » est que celle-ci est en perpétuel renouvellement. Si cette politique de l'Union européenne existe officiellement depuis presque 17 ans maintenant, inaugurée avec le Livre blanc sur la gouvernance de $2001^{3}$, elle a été engagée bien avant, dès les années 1970, par des initiatives de simplification et de codification éparses, de plus ou moins grande envergure ${ }^{4}$. Elle a ensuite connu une évolution remarquable, qui s'est manifestée par l'adoption d'une multitude de textes de soft law tels que communications ${ }^{5}$, lignes directrices, guides de légistique, accords inter-institutionnels ${ }^{6}$, dont les intitulés parfois retentissants étaient destinés à marquer les esprits, à transmettre un message au public, toujours critique face à la prolifération et à la technicité des réglementations européennes. Évoquons les communications relatives à l'élaboration d'une « réglementation intelligente ${ }^{7}$ "affûtée $»^{8}$, créant le programme puis la plateforme « REFIT (Fitness regulatory) »" destinés comme le nom l'indique, à imposer une cure d'amincissement à la loi, grâce à l'évaluation de la législation passée, en vigueur et à venir... Ces textes ont proposé par conséquent des outils, plateformes, principes qui contribuent aujourd'hui à la formation de la science légistique européenne.

Professeure à l'École de droit de la Sorbonne.

P. Albertini, La qualité de la loi - Expériences française et européenne, Mare\&Martin, 2015 ; F. PerAldi Leneuf, S. DE LA RosA (éd.), L'Union européenne ou l'idéal de la meilleure législation, Collection des cahiers de l'IREDIES Pédone, 2013 ; F. PÉRALDI LENEUF (éd.), La légistique dans le système de l'Union européenne, Bruylant, 2012.

Commission européenne : Gouvernance européenne - Un livre blanc, COM/2001/0428 final du 25 juill. 2001.

G. IsAAC, «La codification du droit communautaire », Revue trimestrielle de droit européen, 1977, p. 77.

Communications de la Commission: Simplifier et améliorer l'environnement réglementaire, COM(2002)278 final; Améliorer la réglementation en matière de croissance et d'emploi dans l'Union européenne, COM (2005) 97 final.

Accord interinstitutionnel Mieux légiférer, fOCE 321 du 31.12.2003, p. 1.

Communication de la Commission, : Une réglementation intelligente au sein de l'UE, COM (2010) 543 final.

Communication de la Commission : Pour une réglementation de l'UE bien affûtée, COM (2012) 746 final.

Communication de la Commission : Programme pour une réglementation affûtée et performante (REFIT) : résultats et prochaines étapes. COM (2013) 685 final du 2.10.2013 ; Décision de la Commission du 19.5.2015 établissant la Plateforme REFIT, C (2015) 3261 final. 
Mais si l'action « Mieux légiférer » est déjà une histoire presqu'ancienne, elle n'en est peut-être aussi qu'à ses balbutiements. En effet, le dernier paquet « Mieux légiférer » publié en $2015^{10}$, conçu par la Commission Junker, relance la discussion sur l'opportunité, la mise en œuvre, la légitimité de la politique de l'Union destinée à favoriser une meilleure qualité des lois, le terme «Loi » devant être d'ailleurs appréhendé dans un sens générique puisque la légistique européenne s'applique indifféremment aux actes législatifs et d'exécution, ainsi bien entendu qu'aux actes délégués et ceux adoptés par les États membres. Ici se trouve la nouveauté du sujet et donc la pertinence de son traitement, à laquelle s'ajoute la nécessité de réfléchir à son application comparée en droit interne et international ainsi qu'à sa mise en œuvre au sein de certaines politiques sectorielles de l'Union européenne.

Afin de ne pas empiéter sur les propos qui sont développés dans l'ouvrage, deux catégories de remarques introductives peuvent être formulées : l'une est relative à l'évolution de la finalité de l'action « Mieux légiférer » (1), l'autre est relative à ses implications institutionnelles et politiques (2).

\section{1) L'évolution de la finalité technicienne de l'action « Mieux légiférer »}

Si l'on centre le propos sur son objet, la réglementation, la vocation originelle de « Mieux légiférer » à l'échelon de l'Union est de redonner du sens à celle-ci, dans le mouvement de la réforme plus globale de la gouvernance de l'Union européenne de 2001. Il est nécessaire de rappeler que celle-ci ciblait entre autres outils, les modes de prise de décision et que l'un des groupes de travail avait bien défini la « gouvernance » comme « l'ensemble des règles, processus et comportements touchant la qualité de l'exercice des pouvoirs publics, en particulier, la responsabilité, la lisibilité, la transparence, la cohérence, l'efficience et l'effectivité ${ }^{11}$. Appliquée à l'écriture des textes, cette formule suggère qu'ils soient clairs et accessibles à tous, ce qui impose effort de rédaction, d'intelligibilité, de cohérence.

Cette préoccupation n'est évidemment pas propre au droit de l'Union. Le débat sur la qualité de la législation existe depuis toujours. Portalis fustigeait déjà « les lois inutiles qui affaiblissent les lois nécessaires $»^{12}$, Jacques Ellul a, lui, toute sa carrière contesté « Les lois techniciennes $»^{13}$. En droit constitutionnel français, sont contestées aujourd'hui, au-delà des impératifs traditionnels de clarté, d'accessibilité, les lois «non normatives », celles qui ne commandent plus, mais qui sont «bavardes » au détriment de leur capacité à se faire entendre/comprendre par le citoyen, comme l'analyse Véronique Champeil Desplats dans un ouvrage sur «La qualité de la loi » ${ }^{14}$. L'ensemble des droits européens est concerné, et même sans aucun doute, la conception du droit international.

\footnotetext{
10 Communication sur l'amélioration de la réglementation COM (2015), 215 final ; Proposition d'accord inter-institutionnel relatif à « Mieux Légiférer », incluant un projet d' « Accord Commun » COM (2015) 216 final.

11 Livre blanc sur la gouvernance européen, op. cit.

12 J.-E.-M. Portalis, Discours préliminaire sur le projet de code civil, présenté le $1^{\text {er }}$ pluviôse an IX, Collection : Voix de la Cité, Éditions Confluences, 2004, $78 \mathrm{p}$.

13 J. Ellul, La technique ou l'enjeu du siècle, A. Colin, 1954, réédition Économica, 1990 ; Le système technicien, Calman Lévy, 1977 ; Le bluff technologique, Hachette, 1988 ; L'illusion politique, R. Laffont, 1965. V. aussi le Cahier spécial consacré aux «Lois techniciennes », Les petites affiches, 05 juillet $2007 \mathrm{n}^{\circ}$ 134, p. 19.

14 V. Champeil Desplats, « La normativité de la loi », in P. Albertini (éd.), La qualité de la Loi, op. cit.
} 
De façon commune, la responsabilité, tant des failles de la loi que de son amélioration, revient à l'ensemble des acteurs impliqués, puisqu'elle est un terrain d'affrontement entre l'autorité à l'origine du projet et les législateurs, représentants des intérêts divergents, ou s'il s'agit de textes d'application, entre l'Institution exécutive et les acteurs de la mise en œuvre. La tâche, quoi qu'il en soit est complexe, ses enjeux énormes, institutionnels, politiques, mais plus fondamentalement tournés vers la conception de la démocratie, le modèle de société et donc la conception du droit que l'on veut promouvoir.

Mieux légiférer s'oppose en effet à moins légiférer. Moins légiférer consiste à alléger la tâche du législateur, à contribuer à réduire quantitativement les textes, à restreindre le poids de l'État afin de laisser intervenir d'autres voies de régulation et d'autres acteurs, privés notamment, mais plus difficilement contrôlables et sanctionnables. Cette approche est influencée par l'idéologie libérale britannique de better regulation proposée à la fin des années $1990^{15}$, qui poursuit avant tout une finalité d'efficacité économique.

Mieux légiférer à l'inverse signifie légiférer autrement : réorganiser le travail parlementaire, restreindre ou encadrer le pouvoir d'amendements, veiller à une meilleure mise en application des textes, œuvrer sur la rédaction, mais aussi réviser les dispositifs obsolètes ou inutiles, procéder à des refontes, codification, renforcer les évaluations des projets ou en vérifier la nécessité, l'utilité au regard de principes économiques et sociaux ou sociétaux. Mieux légiférer est avant tout une opération de légistique dont l'effort consiste à associer étroitement travail sur la forme et sur le fond comme l'ont démontré depuis de nombreuses années les travaux de l'école de Genève, portés notamment par Charles-Albert Morand ${ }^{16}$. L'on accorde une place nouvelle à la préparation technique des lois car leur production implique la connaissance, la remontée d'informations, l'établissement d'un diagnostic pour réduire la marge d'incertitude, éviter le «bavardage ». Ce qui rend inévitable le développement d'études ou d'analyses d'impact, ex ante, ex post, la consultation d'experts, la prise d'informations auprès de la société civile.

Si ces préoccupations sont communes à tout ordre juridique, ce qui caractérisel'Union européenne par rapport aux systèmes nationaux, est, selon la Cour des comptes européenne, dans un rapport de $2010^{17}$, qu'au niveau national, la plupart se contentent d'appréhender la législation en préparation et non les actes d'application, les projets de l'exécutif et non ceux du Parlement. Ils s'intéressent en outre à l'évaluation des charges administratives sans se prononcer, ou rarement, sur les enjeux, objectifs, meilleures options et incidences économiques, sociales, environnementales ou liées aux droits fondamentaux. Le droit de l'Union conçoit au contraire l'approche légistique comme une démarche plus large qui appréhende la législation d'amont en aval, de sa production à sa mise en œuvre de façon à la rendre inclusive, représentative de l'ensemble des intérêts et donc plus légitime a priori. C'est le sens de la « réglementation intelligente».

15 Les travaux de la «Better regulation Task Force » ont été publiés sur http://www.cabinetoffice.gov.uk/regulation/economic_analysis/index.asp. 16 Ch.-A. Morand, «L'Obligation d'évaluer l'effet des lois », in Ch.-A. Morand (éd.), Evaluation législative et lois expérimentales, Presses Universitaires d'Aix-Marseille, 1993; Légistique formelle et matérielle, Presses universitaires d'Aix-Marseille, 1999.

17 Cour des comptes européenne, L'analyse d'impact dans les institutions européennes : soutient-elle la prise de décision ?, Rapport spécial n 3 , octobre 2010 
Toutefois, cet enjeu de perfectionnement technique de la production de la réglementation a cédé le pas, à partir de 2008, à celui économique de l'allégement des charges et de la réduction des coûts en faveur des entreprises. L'orientation des évaluations, consultations et autres outils ou instruments de légistique ont été mobilisés à cette fin $^{18}$. Le programme REFIT proposé partir de 2011 énonce par exemple le « top $10 »$ des législations les plus entravantes : et l'on y trouve bien évidemment des textes sur des sujets sensibles en matière sociale, environnementale, ou relatifs à la sécurité des aliments ou des produits industriels. Il s'agit d'en évaluer l'efficience afin de les modifier ou supprimer. Et sans aucun doute ici, le terme « fitness » se trouve tout à fait approprié. Cette évolution du sens de « Mieux légiférer » participe à ce moment-là à un changement de système de valeur dans l'Union européenne et de conception des normes, sous influence britannique, qui met l'accent sur le résultat ou la performance au profit du marché, plutôt que prioritairement sur l’intérêt général.

Cette orientation a conduit rapidement à de vives critiques de la part des syndicats et des ONG qui se sont coordonnés afin de veiller à la mise en œuvre du programme REFIT. Ainsi le 18 mai 2015, plus de cinquante organisations de la société civile ont créé un « Observatoire du mieux légiférer » («Better regulation watchdog »), composé notamment du Bureau européen des consommateurs, de Finance Watch et des Amis de la terre ${ }^{19}$, ce qui conduit à observer que la légistique européenne, plus que jamais, contient un enjeu politique et institutionnel.

\section{2) Les implications politiques de «Mieux légiférer»}

Le paquet publié en 2015 par la Commission européenne actualise doncl'action « Mieux légiférer » mais aussi le débat qui l'entoure. Il consiste en plusieurs textes : une nouvelle communication, un nouvel accord inter-institutionnel ${ }^{20}$, de nouvelles lignes directrices ${ }^{21}$ qui reprennent les outils existants en les sophistiquant, en les approfondissant, voire pour certains aspects, en réorientant la philosophie sous-jacente.

Il ne fait pas de doute que ce nouvel acte reflète le changement de Commission. Il manifeste la volonté de la Présidence Junker, qui a nommé un Vice-président chargé du « Mieux légiférer ${ }^{22}$, de se réapproprier le dossier mené pendant dix ans tambours battant par Manuel Barroso. Forte de sa nouvelle légitimité politique, l'Institution entend laisser sans conteste son empreinte.

On observe ainsi tout d'abord l'affirmation selon laquelle il s'agit non pas de réglementer moins mais mieux.

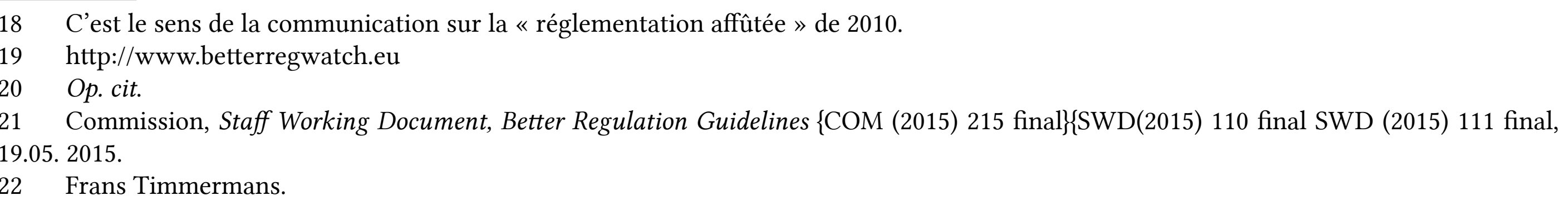


On décèle ensuite, et ce n'est pas la moindre chose en termes de communication, un élargissement du vocabulaire utilisé pour qualifier une bonne législation : en plus des critères classiques de clarté, efficacité, de réduction des charges administratives, celle-ci doit désormais aussi être respectueuse de la subsidiarité, des droits fondamentaux, être de haute qualité.

On remarque également dans le nouvel Accord inter-institutionnel, l'évocation d'une législation qui doit se concentrer sur les domaines où sa valeur ajoutée « est la plus importante pour les citoyens européens », propos compensateurs par rapport aux objectifs antérieurs tournés exclusivement vers l'entreprise et le marché. Par ailleurs, il est créé une nouvelle plateforme intitulée : « alléger la charge-ayez votre mot à dire», canal ouvert à tous.

On constate enfin des éléments nouveaux non négligeables sur la consultation des parties prenantes (élargissement), le renforcement des études d'impact et notamment la valorisation de l'évaluation multi-niveaux, l'ouverture du comité d'examen de la réglementation à des membres extérieurs, le soutien de la Commission au Parlement et au Conseil en la matière, le traitement, enfin, des actes délégués de la Commission, des éléments de rationalisation de la procédure législative, la référence appuyée à l'harmonisation comme unique méthode de simplification, des références beaucoup moins appuyées à l'efficience économique et aux besoins des entreprises, mais plutôt de façon plus générale : un objectif de renforcer la compétitivité et la viabilité de l'économie de l'Union.

À défaut peut-être d'une campagne de communication efficace, le diable étant toujours dans le détail, ces propositions font l'objet toutefois de critiques et même de suspicions. Il est reproché à la Commission en particulier :

- L'inconsistance juridique des principes de légistique et donc la pérennité d'un modèle de droit souple. Autrement dit, à quand un acte juridique, véritable code de légistique, obligatoire et liant juridiquement les institutions?

- Un droit de retrait des propositions législatives par la Commission insuffisamment explicité ;

- L'insuffisante transparence des trilogues ;

- L'absence d'encadrement du lobbying ;

- L'insuffisance de moyens prévus pour le Parlement européen et le Conseil dans la mise en œuvre des études d'impact.

Du côté des suspicions, certains dénoncent la volonté de la Commission « d'édulcorer les réglementations essentielles » et de «subordonner l'intérêt public à celui des multinationales ». Mais l'un des reproches majeurs porte sur l'excès de sophistication globale qui valorise la force de frappe de la Commission au détriment du pouvoir politique. La procéduralisation étoufferait les débats démocratiques au sein du Parlement et du Conseil en détournant celui-ci au niveau de la relation entre la Commission et la société civile. 
Les conséquences n'en seraient pas négligeables. Si la législation acquiert sa légitimité de l'adhésion a priori des acteurs et parties-prenantes, elle serait peut-être plus difficilement contestable a posteriori. Ce qui peut avoir pour effet d'orienter le rôle de la Cour de justice vers un nouveau contrôle de la légistique au détriment du contrôle de la légalité au fond des actes.

Faut-il s'en offusquer? Compte tenu des failles de la représentation démocratique et de la capacité de blocage dont disposent les États aujourd'hui, la recherche d'une autre légitimité est un débat ouvert. Elle permet d'assurer l'acceptation des destinataires en devançant leur attente. Renforcer les consultations pendant l'élaboration de la norme relève par ailleurs des principes de la démocratie participative consacrés dans le TUE à l'article 11.

Quoi que l'on en puisse en penser aujourd'hui, l'on observera objectivement que le processus « Mieux légiférer » relève à la fois de la science, de la technique, de la programmation de l'action publique, de la politique et qu'au travers de ces différentes fonctions, il contribue certainement à un renforcement de la légitimation de l'action de l'exécutif européen avec la complicité de la société civile, plutôt que celui du poids des gouvernements nationaux. Ceci n'est-il pas inéluctable ? Un sujet à suivre de très près dans les années qui viennent. 
Partie I

COMPRENDRE LE «MIEUX LÉGIFÉRER 》 



\section{Chapitre 1}

\section{LE SENS DU « MIEUX LÉGIFÉRER »}





\title{
BETTER REgULATION - VARIATIONS ON A THEME
}

\author{
Francis SNYDER ${ }^{1}$
}

\section{Résumé}

Les origines du "Mieux Légiférer" se situent dans un contexte national britannique spécifique, qui a conditionné son développement dans l'Union européenne et dans les relations internationales de l'UE, par exemple avec la Chine. Ces variations sur un thème commun démontrent que le "Mieux Légiférer" n'a pas pu surmonter les limites de ses origines. En plus, il est à noter que les notions de 'Better Regulation' et 'Mieux Légiférer' sont très différentes, ce qui est encore une indication des difficultés de la transposition des pratiques juridiques.

\section{Abstract}

Better Regulation (BR) originated in a specific national United Kingdom context, which conditioned its initial theoretical and institutional expression and shaped its later development. In the EU, the proponents, idea and practice of BR encountered different opportunities and constraints. The EU's international economic relations with China encompass a conjunction of the legacy of BR, EU realpolitik and a complex and rapidly changing set of economic relations. However, BR has not overcome the limitations of embeddedness to which all social practices, including law-making practices, are subject.

Better Regulation is best viewed as variations on a theme, rather than as an unalterable model that can easily be transplanted from one context to another. This brief article aims to make three main points. First, Better Regulation (BR) originated in a specific national political, social, economic, cultural and legal context, which conditioned its initial theoretical and institutional expression and which then shaped its development after it was transposed to the European Union (EU). Second, in the EU context, the proponents, idea and practice of BR encountered very different opportunities and constraints as compared to its national origins, and it has not been able to overcome the limitations of embeddedness to which all social practices, including law-making practices, are subject. In particular, it has never been

1 C.V. Starr Professor of Law, EU Jean Monnet Chair ad personam and Director, Centre for Research on Transnational Law, Peking University School of Transnational Law, Peking University Shenzhen, China; Emeritus Professor, CERIC, Aix-Marseille University; Visiting Professor, College of Europe, Bruges; Special Endowed Chair of Food Safety, Northwest University of Agriculture and Forestry, Yangling, Shaanxi, China. 
able to achieve many of its original idealistic objectives. Third, these limitations have become especially apparent when BR is considered in the international context. In the context of the EU's international economic relations, we see a conjunction of the legacy of BR, EU realpolitik and a complex and rapidly changing set of economic relations. All of these points turn on the same assertion. Law, understood in the broad sense, is not only part of its social context ('law-in-society' rather than 'law-and-society'), but it is also embedded in its social context, the limitations of which are very difficult to escape. Much may be lost in the translation of legal ideas, institutions and associated social practices from a national context, to the EU context, to the international context.

\section{1) Origins}

Years ago I went into a post office in central London and read with pleasure an announcement about the campaign of the then UK government to make legislation easier to understand, first by ensuring the acts of Parliament and administrative instruments were written as clearly as possible, second by using simple language if possible and third by drawing on the strengths of UK and common law drafting style to spell out legal and other obligations in as much detail as necessary, indeed sometimes using soft law guidelines instead of legislation for this purpose. In 1997 the UK Cabinet Office sponsored the creation of a Better Regulation Taskforce as an independent body to advise the Government. ${ }^{2}$ Almost ten years later, the UK enacted the Legislative and Regulatory Reform Act 2006. ${ }^{3}$ With increasing emphasis on risk regulation as a central function of government, the Better Regulation Commission, growing out of the Task Force, was established in 2006 and was succeeded in 2008 by the Better Regulation Executive. ${ }^{4}$

These developments did not occur in a vacuum. In the UK Margaret Thatcher was leader of the Conservative Party from February 1979 till May 1979 and then Prime Minister from May 1979 till late November 1990. In addition to its laudable objectives with regard to legislative drafting, BR was very much a child of the then Prime Minister's strong orientation towards 'more market and less State', or the so-called 'free market', in which, as Max Weber remarked in his classic Law in Economy and Society ' $[\mathrm{b}] \mathrm{y}$ virtue of the principle of formal legal equality ... the propertied classes ... obtain a sort of "factual autonomy" " The five basic principles which the UK Better Regulation Taskforce identified to improve what it considered to be 'governmental intervention' in the economy were proportionality, accountability, consistency, transparency and targeting; the last principle referred to focusing on the specific problem and minimising side effects. ${ }^{6}$

\footnotetext{
2 See Better Regulation Taskforce, 'Principles of Good Regulation', p. 10, available at http://webarchive.nationalarchives.gov. uk/20100407162704/http:/archive.cabinetoffice.gov.uk/brc/upload/assets/www.brc.gov.uk/principlesleaflet.pdf, last accessed 15 March 2017. See also OECD, Public governance framework for Better Regulation, Executive Summary: United Kingdom, available at http://www.oecd.org/ unitedkingdom/44912018.pdf, last accessed 15 March 2017.

3 Bill 111, Session 2005-2006, cited in Wikipedia, 'Better Regulation Commission', available at https://en.wikipedia.org/wiki/Better_ Regulation_Commission, last accessed 15 March 2017.

4 See J. Black, 'The Role of Risk in Regulatory Processes', in R. BAldwin, M. CAVE and M. Lodge (eds), Oxford Handbook of Regulation (Oxford University Press, Oxford, 2012), pp. $302-349$.

5 M. Weber, Economy and Society, Volume 2 (Guenther Roth and Ckus Wiтtich, eds) (University of California Press, Berkeley, Los Angeles, 1968), p. 699, available at https://archive.org/stream/MaxWeberEconomyAndSociety/MaxWeberEconomyAndSociety_djvu.txt, last accessed 15 March 2017.

6 See Better Regulation Taskforce, 'Principles of Good Regulation', pp. 1, 6, available at http://webarchive.nationalarchives.gov. uk/20100407162704/http:/archive.cabinetoffice.gov.uk/brc/upload/assets/www.brc.gov.uk/principlesleaflet.pdf , last accessed 15 March 2017.
} 


\section{2) Elaboration in the EU}

The European Commission's guidelines on BR state:

'Better Regulation» means designing EU policies and laws so that they achieve their objectives at minimum cost. Better Regulation is not about regulating or deregulating. It is a way of working to ensure that political decisions are prepared in an open, transparent manner, informed by the best available evidence and backed by the comprehensive involvement of stakeholders. This is necessary to ensure that the Union's interventions respect the overarching principles of subsidiary and proportionality i.e. acting only where necessary and in a way that does not go beyond what is needed to resolve the problem.'

These guidelines are very similar to the UK's conception of BR. Such similarity testifies to the legacy of the origins of BR, though in light of the forthcoming Brexit this may appear to be singularly ironic.

In the EU context, however, the original idea and practice of BR encountered very different opportunities and challenges. The lack of transparency and democracy of EU institutions, at least from the citizens' perspective, different regulatory traditions of the EU Member States, the complex quasi-federalist logic of EU legislation and other acts, and the interrelationship in the EU between national, supranational, transnational and international social and economic regulation provided poor soil for the fragile plant of BR.

For example, economists often tell us that European integration involves the integration of three markets: the market for goods and services, the market for factors of production (land, labour, capital) and the market for public policy. If we apply such a micro-economic model to public policy, we might expect that public demand would be met by public authorities who would supply legally binding norms to deal with social problems. However, the EU is as much a regulatory system as it is a legal system, or even more so. All over the world, regulation in contemporary societies usually means governance by experts, not public politics. ${ }^{7}$ This is only one example of the tension between law and regulation, which is an unavoidable aspect of BR.

Better regulation has not really been able to escape its ideological, political and economic origins, but perhaps it did not need to do so. Doubtless, the explanation lies in the politics and economics of European integration. German Ordo-liberal scholars and government officials played a fundamental role in the drafting of the EEC Treaty, the development of EU competition law and the elaboration of EU anti-dumping law. ${ }^{8}$ But the Ordo-liberal perspective provides too limited a conception of the internal market. Even at its origins, EEC law concerned not only with market access but also with

\footnotetext{
7 See F. SNYDER, The EU, the WTO and China: Legal Pluralism and International Trade Regulation, Hart Publishing, Oxford, 2010), Chapter 2, 'Globalisation and the Law', pp. 11-41; T. BÜthe and W. MatruI, The New Global Rulers: The Privatisation of Regulation in the World Economy (Princeton University Press, Princeton NJ, 2013).

8 On anti-dumping law, see F. SNydER, The EU, the WTO and China: Legal Pluralism and International Trade Regulation (Hart Publishing, Oxford, 2010), Chapter 6, 'Global Legal Pluralism and the Creation of New Legal Concepts: the "Non-Market Economy" in EC Anti-dumping Law', pp. 209-264.
} 
market regulation, for example in the field of agricultural policy. ${ }^{9}$ Moreover, from 2004 to 2014 the President of the European Commission was José Manuel Barroso, whose two terms of office embraced the EU's major enlargement, development of the market-based open method of coordination, approval of the Bolkestein directive on the free movement of services and adoption of the Lisbon Treaty. It also witnessed the 'paradox of subsidiarity', of which the principle of proportionality seems to have led to greater use of soft law, reduced judicial control of EU acts, an increased role for market actors and a decline of EU legitimacy. ${ }^{10}$

In the EU it is not surprising perhaps that, as Professor Peraldi-Lefeuf remarked at the conference, ' $\mathrm{BR}$ is a political programme possibly in search of legitimacy. ${ }^{11}$ In the EU context, the idea and practice of BR encountered very different institutional structures, democratic checks and balances and expectations of citizens from those of its country of origin. Nevertheless, it is depressing to note that in the EU BR has never been able to achieve most of its original, idealistic, non-economic and citizen-oriented objectives. So far as the citizen is concerned, the EU BR initiative remains a theoretically admirable but in practice a largely unattained and probably unattainable ideal. In a 'market without a State', ${ }^{12}$ to what extent is it possible to simplify complex regulatory law and make it clearly understandable by citizens? What does 'clarity' mean in the context of a multi-national, multi-lingual polity without a real political public sphere? One should also ask: 'clarity' for whom? It is well-known that clarity for a lay person may well be ambiguity for a lawyer, and clarity for a lawyer is likely to be overly technical and overly complex, if not incomprehensible, for a lay person.

\section{3) Competing Conceptions of Regulation}

When BR crossed the Channel, it also encountered strikingly different conceptions of regulation. Terminology is one indication. For example, Better Regulation (BR) is usually translated into French as Mieux légiférer (ML). However, the two expressions have very different associations in their respective legal cultures and legal systems. BR derives from British, common law thinking about the flexible use of many types of norms to achieve desired social objectives. ${ }^{13} \mathrm{ML}$ stems from continental civil law systems, notably French law, with its emphasis on centralised government, legislation and very different conceptions of regulation.

We can distinguish four different conceptions of regulation upon which BR and ML drew in various settings. First, in its original conception in the United States, regulation, at first economic regulation and later social regulation, referred to governmental intervention in the market which was deemed to be necessary to protect public goods as a result of market failures. In other words,

\footnotetext{
9 See e.g. F. Snyder, 'CAP' in Erik Jones, Anand Menon and Stephen WeAtherill (eds), Oxford Handbook of the European Union (Oxford University Press, Oxford, 2012), pp. 484-495.

10 See F. SNyder, 'Soft Law and Institutional Practice in the European Community', in S. Martin (ed), The Construction of Europe: Essays in Honour of Emile Noël (Kluwer Academic Publishers, Dordrect, 1994), pp. 197-226.

11 Based on my notes taken in English of Professor Peraldi-Leneuf's presentation in French at the conference.

12 See the work of C. Joerges, F. Scharf and also J. CAporaso and S. TARrow, 'Polanyi in Brussels: European Institutions and the Embedding of Markets in Society', RECON Online Working Paper 2008/01, available at http://www.reconproject.eu/projectweb/portalproject/ RECONWorkingPapers.html, last accessed 6 November 2016.

13 See for example C. Scott, Regulation (Routledge, London, 2003).
} 
the market was the desirable starting point or state of affairs, but it was not able to provide or protect certain public goods such as a good environment and healthy food. As a result of such market failures, government intervention was necessary, for example in the form of legislation or judicial decisions.

A second conception is the Marxist or neo-Marxist approach developed in France by Robert Boyer. This macroeconomic perspective is concerned with 'an examination of geographical and historical variations in the institutional arrangements that define capitalist economies. ${ }^{14}$ As Boyer and Saillard point out, among users of the English language the French term régulation was often 'confused with regulation (règlementation in French) ; furthermore, as a result of conservative deregulation strategies [such as the Thatcher revolution] English usage of the term 'regulation' has experienced a revival'. ${ }^{15}$ Clearly, this second conception of regulation involved completely different assumptions and questions and reached different conclusions from those of the first conception of regulation.

A third conception is the 'droit de la régulation'founded in France by Marie-Anne Frison-Roche. It imports from American scholars the idea that governmental intervention, usually in the form of legislation, is necessary in case of market failure. However, it adds that certain sectors, such as public health, should not be governed by the market but require governmental action in the public interest to preserve a balance between market efficiency and public interest, including fundamental rights. ${ }^{16}$ It thus weaves together two strands: first, the idea that market failure requires governmental intervention in the market, usually in the form of legislation, and second the idea that certain areas of activity should not be left to the market. It also assumes that the government represents the public interest, an assumption was not necessarily a feature of the first conception of regulation.

A fourth conception of regulation is that developed primarily at the London School of Economics and the Australian National University School of Regulation and Global Governance (RegNet), with considerable faculty exchange and joint publications. According to this conception, regulation is the use of numerous institutions, tools and techniques to ensure the achievement of desired social goals. ${ }^{17}$ It does not assume the free market as ideal, or that governmental regulation stems mainly from market failure, or that the markets is inevitably deficient in providing public goods. Regulation is not limited to the government; it can be and frequently is redistributed among many actors and institutions, including private actors. Nor is it limited to legislation, or even to law or formal norm-making processes. From this perspective, many institutions, not only the State, may represent the public interest. ${ }^{18}$

These different conceptions of regulation often remain hidden, implicit and unarticulated in the expression 'Better Regulation'. They testify to two tensions: one between law and regulation, and the

14 R. Boyer, The Regulation School: A Critical Introduction, trans. C. Charney (Columbia University Press, New York, 1990); R. Boyer, 'Introduction', in R. Boyer and Y. SAILlard, Regulation Theory: The State of the Art (Routledge, London, 2002), p. 2.

15 R. Boyer, 'Introduction', in R. Boyer and Y. SaIllard, Regulation Theory: The State of the Art (Routledge, London, 2002$),$ p. 1.

16 M.-A. Frison-Roche, Les 100 mots de la régulation (Presses Universitaires de France, Paris, 2011).

17 For an EU example, see F. SNYDER, 'The Effectiveness of European Community Law: Institutions, Processes, Tools and Techniques', Modern Law Review, 56, 1, January 1993, 19-54.

18 For representative publications, see J. BLACK, Rules and Regulators (Clarendon Press, Oxford, 1997); J. BrAIthEwAITE and P. DraHos, Global Business Regulation (Cambridge University Press, Cambridge, 2000); C. ScotT (ed), Regulation (Routledge, London, 2003); P. DraHos (ed), Regulatory Theory: Foundations and Applications (Australian National University Press, Acton ACT, 2017), available at http://press-files.anu.edu. $\mathrm{au} /$ downloads/press/n2304/pdf/book.pdf?referer=2304, last accessed 16 March 2017. 
other concerning the relative scope of law and regulation. Each of the four conceptions or schools of regulation views these tensions in a different way. Let us take the example of the LSE/ANU conception, to which I subscribe and indeed have contributed. From this standpoint, regulation is a process. It may or may not require legislation. The law in the general sense of legally binding measures or in the narrower sense of legislation may be used an instrument of regulation. But regulation may and often does involve soft law, in the EU and elsewhere. ${ }^{19}$ In addition, regulation cannot be reduced to proceduralisation, in the sense of agreement on procedures to be followed and respected in situations when the parties cannot agree in advance on ultimate outcomes. But this conception of regulation does not require that agreement on procedure replaces agreement on outcomes. Parties may agree on outcomes, or they may agree simply on procedures, or they may agree on procedures, including agreement to accept the outcome of procedures. It may involve both agreement on procedures and prior agreement on substantive outcomes. Such processes may involve the State but they do not necessarily do so. From this standpoint, BR seems very distant from a classic understanding of ML.

A similar example stems from the observation that the main problem of BR is that it leads to a 'negotiated law'. However, this feature may be inherent in regulatory law, at least according to the fourth conception of regulation. The command-and-control model of regulation is out-dated and often ineffective, especially in democratic societies. Many current theories of regulation recognise the significance and frequency of reflexive law and of transnational normative repertoires. In the former, which has long been recognised as a principal characteristic of economic law and regulation, first perhaps in the UK, the basic rules are negotiated between regulator and regulatee. In the latter, domestic and EU rule-makers draw from a range of concepts, principles, rules and procedures which have been generated by national and international legal processes. Both may be seen as aspects of 'negotiated law'. It would be unusual if BR were any different.

Finally, regulation is not limited to the national legal system or domestic political arena. Indeed, there is an enormous academic literature on international and transnational regulation..$^{20}$ Many scholars of WTO law regard the WTO institutional and normative system as a regime for regulating international trade. The WTO is a good example of the globalisation of local practices, notably from the United States and the EU. The norms, procedures, assumptions and legal culture of WTO law draw heavily on American international trade law, and decision-making concerning standardisation or anti-dumping borrows from US administrative law concepts and principles such as transparency, coherency, consultation, evidence-based decision-making, minimum cost and least administratively burdensome. International trade regulation is a prime illustration of proceduralisation. In this context, such a policy choice represents a choice that quasi-judicial institutions rather than diplomatic negotiations will settle disputes, particularly because the WTO agreements are the result of international negotiations and compromise and hence often deliberately vague or ambiguous, even though of course they are legally binding.

19 For example, on China see H. Luo and G. Song, Soft Law Governance: Towards an Integrated Approach (William S. Hein, New York, 2013).

20 See for example F. SNYDER and L. YI (eds), The Future of Transnational Law: EU, USA, China and the BRICS / L'avenir du droit transnational: UE, USA, Chine et les BRICS (Bruylant, Brussels, 2015). 


\section{4) Better regulation, the EU and International Economic Law}

As the preceding paragraph suggests, some aspects of BR are relevant to international economic law, despite the very different context. However, if the translation of BR to the EU and relations among Member States was difficult, its potential transposition to the radically different context of international economic relations has been fraught with difficulty. The change of context and numerous conflicts of interest, especially with the transformation of international economic relations in recent decades, have undercut whatever potential application BR and its companion REFIT might have had. An instructive example is the current negotiations with China concerning the non-market methodology in EU anti-dumping law.

These negotiations focus on Section 15 of the Protocol of Chinese Accession to the World Trade Organisation, which concerns price comparability in determining subsidies and dumping. ${ }^{21}$ The most controversial provision states that:

'(d) Once China has established, under the national law of the importing WTO Member, that it is a market economy, the provisions of subparagraph (a) shall be terminated provided that the importing Member's national law contains market economy criteria as of the date of accession. In any event, the provisions of subparagraph (a)(ii) shall expire 15 years after the date of accession. In addition, should China establish, pursuant to the national law of the importing WTO Member, that market economy conditions prevail in a particular industry or sector, the non-market economy provisions of subparagraph (a) shall no longer apply to that industry or sector'.

Section 15 expresses a complex, negotiated international compromise among numerous conflicting interests instead of the clarity which might have been possible by virtue of the original ideas of BR. The basic issue in disagreement is whether this provision allows other WTO Members to continue to use their current non-market economy methodology in case of imports from China until specific conditions are satisfied, or whether the provision grants China market economy status (MES) automatically as of 12 December 2016. Both sides of the debate use economic, political and legal arguments.

Unfortunately, many recent commentators seem to neglect the basic methods of interpretation of public international law. As set forth in Article 31 of the Vienna Convention on the Law of Treaties: 'A treaty shall be interpreted in good faith in accordance with the ordinary meaning to be given to the terms of the treaty in their context and in the light of its object and purpose'.22 The EU lawyer may note that these methods of interpretation are the reverse of those expressed by the Court of Justice of the European Union since Case 26/62 Van Gend en Loos. The recent proposal

\footnotetext{
21 World Trade Organization, Protocol on the Accession of the People's Republic of China, Decision of 10 November 2001, WT/L/432, 23 November 2001, available at https://www.wto.org/english/thewto_e/acc_e/completeacc_e.htm\#chn, last accessed 16 March 2017.

22 Vienna Convention on the Law of Treaties, Article 31(1), available at https://treaties.un.org/doc/publication/unts/volume\%201155/ volume-1155-i-18232-english.pdf, last accessed 16 March 2017.
} 
by the European Commission to reform EU anti-dumping law ${ }^{23}$ attempts to achieve a reasonable compromise among numerous conflicting interests and objectives. Space limits prohibit an analysis of the proposal here. It is noteworthy, however, that the proposal is based on consultation of stakeholders, including China, and on an impact assessment, both of which are principal characteristics of BR. ${ }^{24}$ It is not clear what weight was given to consumers, and the proposal remains true to the Ordo-liberal origins of EU anti-dumping in failing to recognise Chinese State capitalism as one of the varieties of capitalism. Nevertheless, it represents an effort to steer between the Scylla of long-standing protectionism and the Charybdis of widespread loss of EU jobs.

This brief paper focused on origins, development and application of Better Regulation, tracing similarities and differences among several variants of BR. It emphasised the importance of the contexts of law. BR focuses mainly on markets, partly because of its ideological and political origins and partly because of the mainly economic orientation of European integration and the EU. However, the original promises of BR concerned not simply market actors but also citizens. If BR is to fulfil its original promises, we need to rethink its current orientation.

How can we escape this market focus, and how can we develop a perspective which takes account of recent economic and political developments? Three points stand out. First, the most powerful market actors today are organised global value chains, including many SMEs which form part of international production networks or international supply chains. Second, market economies produce winners and losers, unless BR or other forms of regulation embody strong redistributive policies. Third, the basic principles underlying BR should consist not simply of a focus on procedures but also, and more broadly, of social solidarity ethics, namely development, respect for the environment and social justice. ${ }^{25}$ These crucial points should be considered seriously in reshaping BR or similar normative processes in the interests of citizens.

\footnotetext{
23 European Commission, Proposal for a Regulation of the European Parliament and of the Council amending Regulation (EU) 2016/1036 on protection against dumped imports from countries not members of the European Union and Regulation (EU) 2016/1037 on protection against subsidised imports from countries not members of the European Union, $\{\operatorname{SWD}(2016) 370$ final $\},\{\operatorname{SWD}(2016) 371 \mathrm{final}\}$, \{SWD(2016) 372 final\}, COM(2016) 721 final, 2016/0351(COD), Brussels, 9 November 2016, available at http://trade.ec.europa.eu/doclib/docs/2016/november/ tradoc_155079.pdf, last accessed 16 March 2017.

24 See also European Commission, Trade, New Archive, 'Commission proposes changes to the EU's anti-dumping and anti-subsidy legislation', available at http://trade.ec.europa.eu/doclib/press/index.cfm?\&id=1573, last accessed 16 March 2017.

25 On social solidarity ethics, see F. SNYDER, The EU, the WTO and China: Legal Pluralism and International Trade Regulation (Hart Publishing, Oxford, 2010), Chapter 10, 'Social Solidarity Ethics and the WTO', pp. 381-423.
} 


\title{
LA CLARTÉ RÉ DACTIONNELLE EN PRATIQUE
}

\author{
Jennifer Gracie LL.B. ${ }^{1}$
}

\section{Résumé}

Il est reconnu qu'un des aspects d'une meilleure législation, est la législation rédigée de façon claire. Par conséquent, les législations européenne et nationale exigent de plus en plus la clarté rédactionnelle. Mais qu'est-ce que «clarté » signifie ? Si on ne peut pas la définir, comment peut-on y parvenir ? Et si la législation même n'en donne pas un bon exemple, ne serait-il pas irrespectueux pour ses lecteurs qu'elle exige la clarté ? Cet article examine ces questions et propose une solution.

\section{Abstract}

It is recognised that one aspect of better legislation, is legislation that is drafted clearly. As a result, clear drafting is increasingly being required by European and national legislation. But what does 'clear' mean? If it cannot be defined, then how can it be achieved? And if the legislation itself is not a good example of clear drafting, then would it not be disrespectful of its readers to require clarity? This article explores these questions and suggests a possible solution.

Pour examiner le côté pratique de la clarté rédactionnelle de la législation européenne il faut poser deux questions principales : Qu'est-ce que la clarté rédactionnelle (1) et Comment rédiger d'une façon claire (2) ?

\section{1) Qu'est-ce que c'est la clarté rédactionnelle?}

...est-ce la « simplification »?

...récemment on parle plutôt de « Easification » ou « facilification »

...sinon, « clarification », « restylisation », « fitness » ou

...« modernisation $» ? .$.

$\overline{1}$ Solicitor (non-practising) \& ancien Membre du conseil de l'ordre de la Law Society de l'Angleterre et du Pays de Galles, Chercheur Associé de l'Institut des Hautes Études sur la Justice (Paris). 


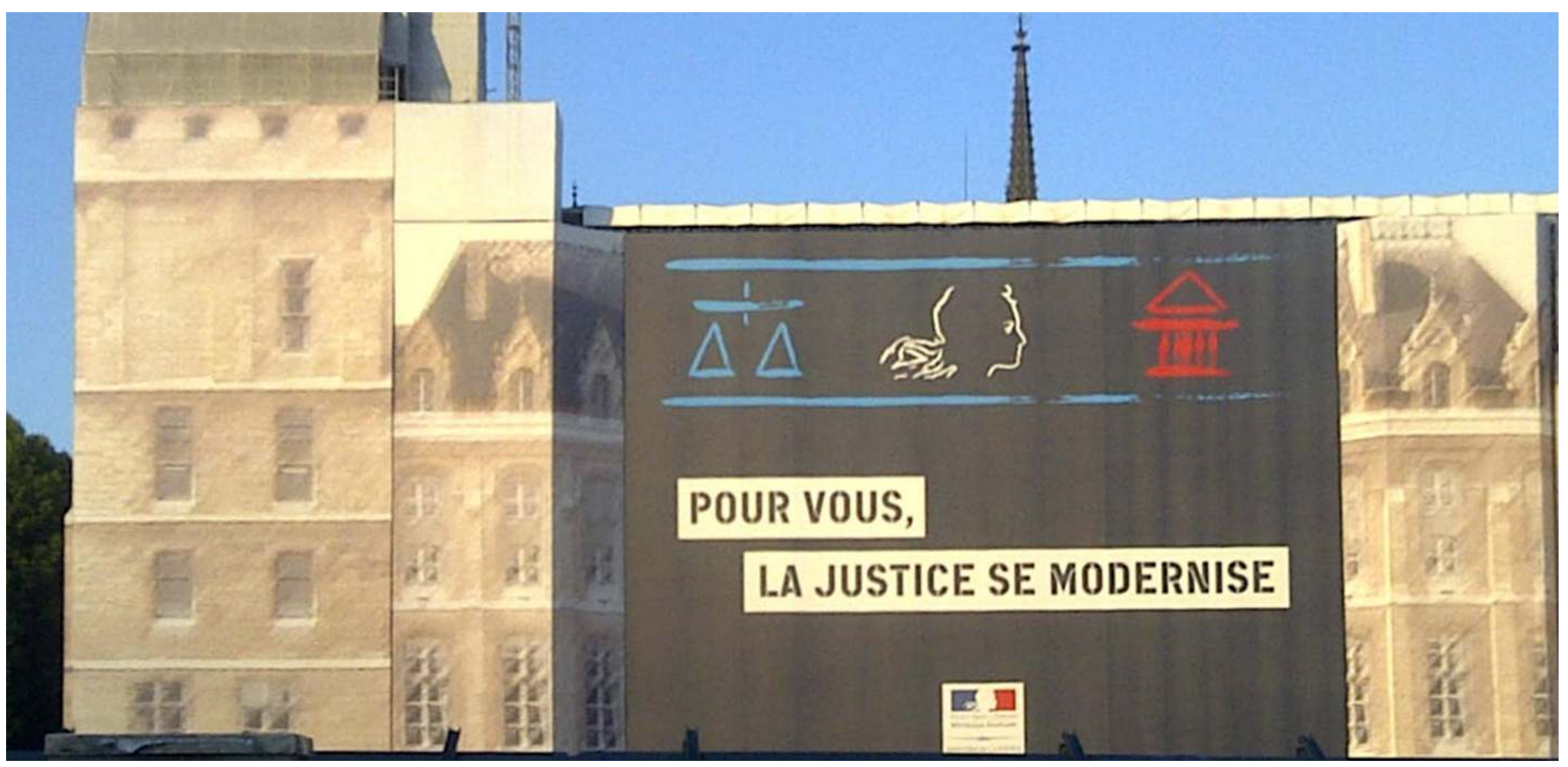

Le Palais de Justice, Paris, 2011.

ll me semble que personne n'est d'accord :

- $\quad$ soit il n'y a pas de définition du tout - on présume qu'on sait tous ce que c'est ;

- soit la définition tourne en rond sans jamais identifier le concept : il faut être lisible pour être clair ; pour être clair, il faut être intelligible. Il faut être clair et intelligible - ou intelligible, mais pas clair - sans expliquer pourquoi.

Par exemple :

\subsection{En France}

Dans le document de travail du Sénat La Qualité de la Loi de $2007^{2}$, il est fait référence à l'abandon par le Conseil constitutionnel du principe de clarté de la loi « très récemment, compte tenu de ses ambiguïtés, fréquemment relevées par la doctrine. ». Et à la place « l'objectif d'intelligibilité est la norme de référence unique en la matière. ».

...alors plus de clarté, mais de l'intelligibilité...

...la différence étant? Pas claire !

$2 \quad$ Note de synthèse du service des études juridiques $n^{\circ} 3$ (2007-2008) - 1er $^{\text {er }}$ octobre $2007:$ http://www.senat.fr/ej/ej03/ej03.html 


\subsection{En Europe}

\section{Préface du Guide Pratique Commun des institutions Européennes de $2013^{3}$ :}

«Afin que la législation communautaire soit mieux comprise et correctement mise en œuvre, il est essentiel de veiller à sa qualité rédactionnelle. En effet, pour que les citoyens et les opérateurs économiques puissent connaître leurs droits et obligations et les juridictions les faire respecter, et pour que, là où elle s'impose, une transposition correcte et dans les délais soit effectuée par les États membres, les actes adoptés par les institutions communautaires doivent être formulés de manière intelligible et cohérente, et suivant des principes uniformes de présentation et de légistique. »

Ceci est un bon exemple pour démontrer que ceux qui exigent la cohérence et la clarté ne se sont pas mis d'accord, au préalable, sur ce que ces mots signifient. Une phrase (la deuxième) de 67 mots indique que la phrase n'est pas aussi claire qu'elle devrait être. Et qu'est-ce que le mot « cohérente » ajoute au mot « intelligible» ? À mon avis, la cohérence forme une partie de l'intelligibilité.

On ne trouve pas non plus de cohérence dans les termes utilisés dans le Guide. À la page 10, par exemple, il n'y a pas de référence à « intelligible » ni à « cohérente ». À la place, on parle de claire, simple et précise :

«1. Les actes législatifs communautaires sont formulés de manière claire, simple et précise.

La rédaction d'un acte législatif doit être :

- Claire, facile à comprendre, sans équivoque,

- Simple, concise dépourvue d'éléments superflus

- Précise, ne laissant pas d'indécision dans l'esprit du lecteur. »

Quelle est la différence entre les mots « claire » et «précise »?

Les définitions données ici n’aident pas. « Claire » est censée être « sans équivoque »; " précis » signifie «ne laissant pas d'indécision dans l'esprit du lecteur ». La différence ? D’après moi, il n'y en a pas - c'est le même concept ! Tout simplement, « sans équivoque » s'applique au rédacteur et «ne laissant pas d'indécision » s'applique au lecteur.

$\overline{3}$ http://eur-lex.europa.eu/content/techleg/FR-guide-de-redaction-legislative.pdf 


\section{L'Accord interinstitutionnel 2016 (AII 20016) « Mieux légiférer $»^{4}$}

On aurait espéré des améliorations en 2016, dans le dernier accord interinstitutionnel, mais non :

AII 2016, préface (au paragraphe 2) :

« Les trois institutions reconnaissent qu'elles ont conjointement la responsabilité d'élaborer une législation de l'Union de haute qualité et de veiller à ce que ladite législation se concentre sur les domaines où sa valeur ajoutée est la plus importante pour les citoyens européens, à ce qu'elle soit aussi efficace et effective que possible pour atteindre les objectifs stratégiques communs de l'Union, à ce qu'elle soit aussi simple et claire que possible, à ce qu'elle évite la réglementation excessive et les lourdeurs administratives pour les citoyens, les administrations et les entreprises, en particulier les petites et moyennes entreprises (PME), et à ce qu'elle soit conçue de manière à faciliter sa transposition et son application pratique ainsi qu'à renforcer la compétitivité et la viabilité de l'économie de l'Union ».

Cette phrase de 125 mots explique que la responsabilité des 3 institutions est de veiller à ce que la législation « soit aussi simple et claire que possible».

AII 2016, Article 2 (au paragraphe 2) : Cet article explique que les institutions européennes «... conviennent de promouvoir la simplicité, la clarté et la cohérence dans la rédaction de la législation de l'Union».

Ici on voit le mot « cohérence » ajouté aux mots « simplicité et clarté ». On a vu le mot « cohérent » ajouté au mot « intelligible » dans la préface du Guide européen de 2013. Ici, c'est ajouté à la « simplicité et clarté ». Je répète donc ma question : qu'est-ce que le mot «cohérente » ajoute au mot « intelligible » ou, ici, « clarté » ? Pour moi, la cohérence forme une partie de l'intelligibilité et de la clarté.

J'ajoute une autre question. Quelle différence y a-t-il entre « clarté » et « intelligibilité »? D’après moi, ces mots expriment le même concept du point de vue du :

- lecteur (intelligibilité) ;

- rédacteur (clarté).

Continuons...

AII 2016, Article 3 (56 mots) :

«Les trois institutions conviennent que la législation de l'Union devrait être compréhensible et claire, permettre aux citoyens, aux administrations et aux entreprises de comprendre aisément leurs droits et leurs obligations, prévoir des exigences appropriées en matière d'information, de suivi et d'évaluation, éviter la réglementation excessive et les lourdeurs administratives, et être aisée à mettre en œuvre ». 
Faisons une petite analyse des articles 2 et 3 :

- On trouve « compréhensible » dans l'article 3 au lieu d' « intelligible » dans l'article 2.

- Regardons le sens de ces deux articles : y a-t-il une différence ou est-ce une répétition? Je dirais que les deux articles disent la même chose. La répétition est un signe de manque de clarté.

Voici deux directives qui démontrent que les personnes qui aspirent à la clarté n’y parviennent pas :

- Directive 2005/29/CE du Parlement européen et du Conseil du 11 mai 2005, Article 7 (Omissions trompeuses) : «2. Une pratique commerciale est également considérée comme une omission trompeuse lorsqu'un professionnel [...] dissimule une information substantielle [...] ou la fournit de façon peu claire, inintelligible, ambiguë ou à contretemps [...] ». La phrase entière fait 85 mots.

Un texte ambigu n'est pas clair. Une phrase de 85 mots non plus ! Mais pourquoi utiliser 2 mots, 3 ou 4-même, quand un mot serait suffisant?

- Directive 2011/83/UE du Parlement européen et du Conseil du 25 octobre 2011 : Article 8 : Obligations formelles concernant les contrats à distance : «2. [...] le professionnel informe le consommateur d'une manière claire et apparente, et directement avant que le consommateur ne passe sa commande. 3. Les sites de commerce en ligne indiquent clairement et lisiblement [...]».

Même les deux alinéas qui se suivent n'utilisent pas les mêmes termes !

Il me semble donc qu'il n'existe pas de définition adéquate actuellement pour répondre à la question « qu'est-ce que la clarté ? ». Je passe donc à la deuxième question.

\section{2) Comment rédiger de façon claire?}

\subsection{Définition de « rédaction claire »}

Il est impossible de déterminer comment rédiger d'une façon claire, sans se mettre d'accord sur une définition. Mais les pays en Europe ainsi que les institutions européennes essaient de faire.

Prenons un exemple, la Commission européenne a développé des listes pour encourager la rédaction des textes clairs. Cette brochure de 2015 (publiée depuis 2010) intitulée Rédiger clairement ${ }^{5}$ existe en 28 langues. À la page 2, le conseil n 4 est : « soyez clair et concis ». Il faut donc être clair pour être clair!

Vu de près (à la page 6, la première colonne intitulée « Clair... »), ce conseil consiste à « Utilisez des mots simples ». Et donc, « simple » c'est être « clair».

5 Commission européenne, Direction générale de la traduction ISBN: 978-92-79-46900-8 https://bookshop.europa.eu/fr/r-diger-clairementpbHC0215257/ 
Cependant, selon son Guide Pratique Commun de 2013 (voir 1.2.1), « simple » signifie « concis »!

Mais dans Rédiger clairement, la deuxième colonne à la page 6 est intitulée « concis », comme si cela était différent de « clair » :

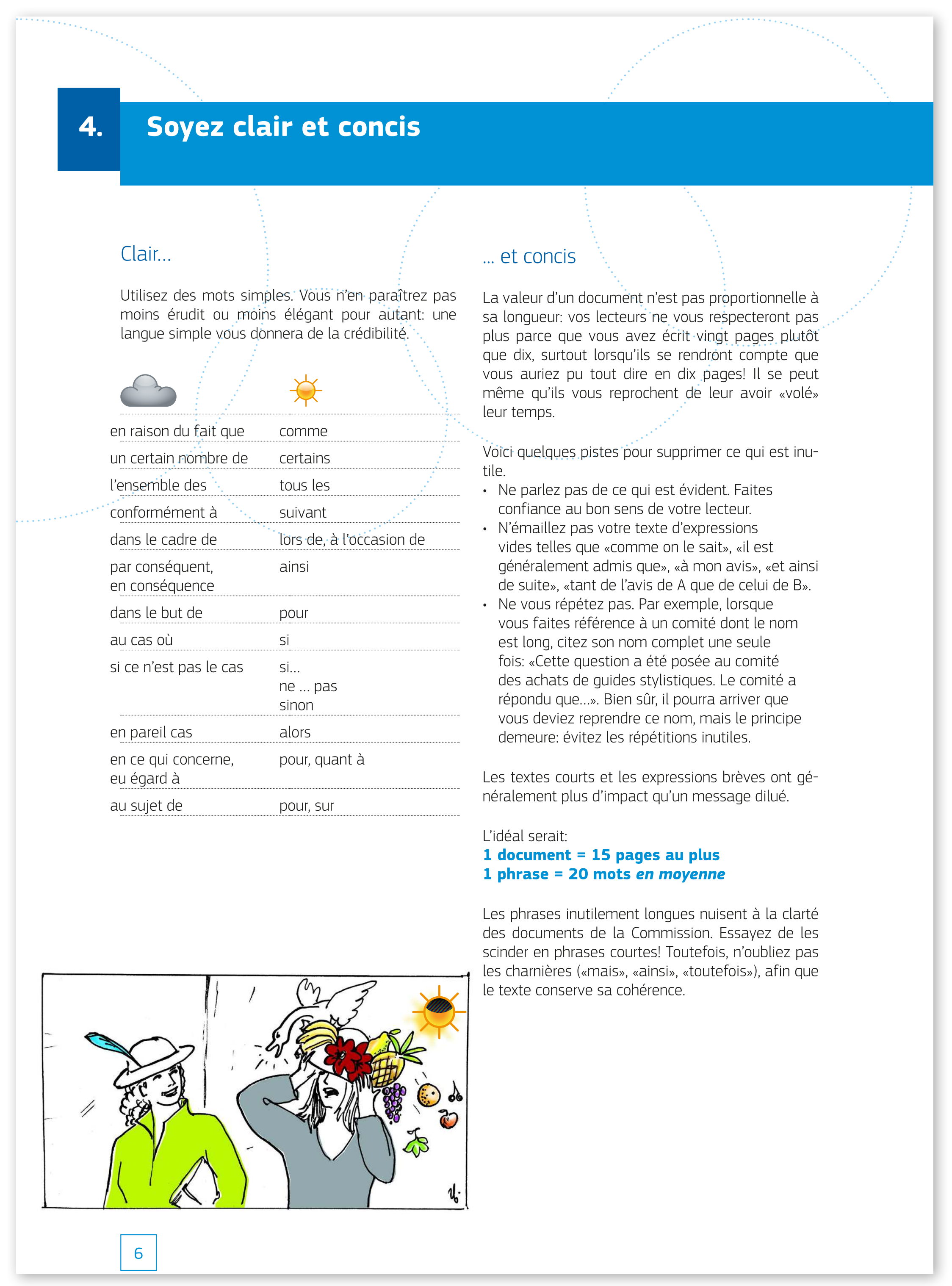

\section{C'est clair?!}

Sans définitions adéquates, sans cohérence dans l'utilisation des termes, avec des répétitions, ni le Guide de 2012, ni l'accord interinstitutionnel de 2016, ni ces brochures de « rédaction claire », ni les directives ne peuvent être clairs eux-mêmes. La législation devrait elle-même donner l'exemple, surtout lorsqu'elle préscrit la clarté. 


\subsection{Soyons réalistes}

Si les exigences ne sont pas réalistes, les objectifs ne seront pas atteints - et les rédacteurs ainsi que les lecteurs vont vite être déçus :

\section{Soyons réalistes par rapport aux rédacteurs}

Il y a un obstacle important à franchir : la culture juridique. Les membres de la famille juridique, pour protéger leur culture - ne veulent pas qu'on touche à leur langue - qu'ils prétendent - que nous prétendons - maîtriser.

D’après mon expérience, les listes linguistiques n'aident pas. Elles sont une sursimplification. Les juristes les citent pour rejeter toute amélioration ! Il faut reconnaitre qu'écrire un langage est plus complexe que de le parler. Banaliser l'écriture juridique par des listes - n'est qu'une diversion.

Citons le Professeur Pinker, Professeur de Psychologie à Harvard, dans son livre The Sense of Style ${ }^{6}:$ "The spoken word is older than our species...But the written word is a recent invention that has left no trace in our genome and must be laboriously acquired throughout childhood and beyond.»

Formuler les idées et les mettre dans un ordre logique forme $80 \%$ du travail de clarté - au minimum. Pour reformuler les idées juridiques, il faut comprendre les concepts juridiques.

\section{Soyons réalistes aussi par rapport aux lecteurs}

La longueur idéale d'une phrase est liée au nombre de mots que le cerveau retient dans sa mémoire de travail pendant la lecture. Au-delà d'un certain nombre de mots, la compréhension devient difficile et nous devons relire le début de la phrase.

Par rapport aux textes juridiques, est-il possible que les citoyens comprennent les concepts juridiques sans être formés en droit ? Au-delà d'un certain point, non, dirai-je. Mais cela ne veut pas dire qu'il ne faut pas essayer de rendre le texte aussi clair que possible. Ce qui nous mène à la solution...

\subsection{La solution pour la clarté rédactionnelle}

La preuve par l'exemple : le projet de réécriture aux États-Unis des règles fédérales de procédure civile et des règles fédérales de la preuve. Ce travail a été fait par une commission du Gouvernement américain nommée la « Judicial Conference Advisory Committee », dirigée par un professeur de droit, Joe Kimble. Il a remporté deux prix prestigieux (Burton Awards) en 2007 et 2011 pour la réforme de la loi.

$6 \quad$ Pinker, S. (2014). The Sense of Style: The Thinking Person's Guide to Writing in the 21st Century. New York, NY: Penguin. 
Voici une règle fédérale de la preuve qui a été réécrite (Règle 104 sur les questions préliminaires) :

Avant

(c) Hearing of jury. Hearings on the admissibility of confessions shall in all cases be conducted out of the hearing of the jury. Hearings on other preliminary matters shall be so conducted when the interests of justice require, or when an accused is a witness and so requests.

[47 mots]
Après

(c) Conducting a Hearing So That the Jury Cannot Hear It. The court must conduct any hearing on a preliminary question so that the jury cannot hear it if:

(1) the hearing involves the admissibility of a confession;

(2) a defendant in a criminal case is a witness and requests that the jury not be present; or

(3) justice so requires.

[60 mots]

La commission a retravaillé le contenu des phrases. Ce n'est pas possible sans compréhension des concepts du droit.

Rendre clair ne veut pas forcément dire que chaque phrase doit être plus courte. Regardons l'exemple ci-dessus : il y a 47 mots dans la version originale à gauche et 51 dans la version réécrite à droite.

Néanmoins,un texte qui a été réécrit est souvent plus court que l'original. Un des aspects de l'écriture claire étant d'enlever les répétitions ou - grâce aux idées - dire la même chose plus succinctement. La règle 610 réécrite en est un exemple :

Avant

Evidence of the beliefs or opinions of a witness on matters of religion is not admissible for the purpose of showing that by reason of their nature the witness' credibility is impaired or enhanced.

\section{Après}

Evidence of a witness's religious beliefs or opinions is not admissible to attack or support the witness's credibility.

[18 mots]

[34 mots]

Les règles réécrites sont plus facile à lire. Mais elles ne sont pas plus facile à écrire. Cela n’a été possible que par la reformulation des idées. Avec une maîtrise suffisante des concepts contenus dans le texte ainsi que l'accord de l'équipe juridique (forcément, avec des compromis).

Ce travail a permis d'enlever la couche de complexité qui était superflue. Cette couche superflue rendait plus difficile la lecture, et par les non initiés en droit, et par ceux dont l'anglais n'est pas la langue maternelle.

Quel est l' « impact » (pour utiliser ce terme populaire) d'une telle rédaction claire ? La version réécrite des règles fédérales de la preuve avait $14 \%$ de mots en moins que l'originale. « Time is money». Qui a le temps de lire $14 \%$ de plus que nécessaire ? Et de plus, qui va payer pour ce temps perdu ?

Au niveau européen : si la législation est, disons, $14 \%$, plus longue que nécessaire et traduite 28 fois, le volume devient vite ingérable. Mais c'est ce qu'on est en train de vivre - ou plutôt, de souffrir ! Ceci joue forcément sur la popularité - ou le manque de popularité - des institutions européennes. 
Et il ne faut pas oublier ce que Portalis a dit au sujet du Code Napoléon en 1801 : «Quand la loi est claire, il faut la suivre ; quand elle est obscure, il faut en approfondir les dispositions ${ }^{7} »$. Portalis n'était pas concerné par la globalisation. De nos jours, au moment de la rédaction, il faut penser à sa clarté, non seulement pour ne pas perdre le sens prévu (y compris ambigu, le cas échéant) par le rédacteur, mais pour réduire les problèmes de traduction.

N'oublions pas non plus que, comme constaté par le Conseil Constitutionnel, la clarté de la législation fait partie des droits de l'homme :

« [...] il appartient au législateur d'exercer pleinement la compétence que lui confie l'article 34 de la Constitution; qu'il doit, dans l'exercice de cette compétence, respecter les principes et règles de valeur constitutionnelle [...] qu'à cet égard, le principe de clarté de la loi, qui découle de l'article 34 de la Constitution, et l'objectif de valeur constitutionnelle d'intelligibilité de la loi, qui découle des articles 4, 5, 6 et 16 de la Déclaration des droits de l'homme et du citoyen de 1789, lui imposent, afin de prémunir les sujets de droit contre une interprétation contraire à la Constitution ou contre le risque d'arbitraire, d'adopter des dispositions suffisamment précises et des formules non équivoques $[\ldots]^{8}$. »

La solution est donc d'exiger un devoir aux rédacteurs de toute législation (européenne et autre) envers les lecteurs. Un devoir qui consisterait à enlever toute couche de complexité superflue. Couche qui fait perdre énormément de temps et d'argent aux entreprises comme aux particuliers, notamment à cause des traductions superflues qui en résultent...

Et donc oubliez les listes linguistiques pour rendre des textes juridiques plus clairs. Par exemple, si on remplace des mots complexes par des mots simples, le texte devient-il clair pour autant ? Non, dirais-je, comme beaucoup d'autres juristes d'ailleurs. Mon raisonnement est :

- ce n'est pas toujours possible (certains mots complexes sont requis par la loi),

- ce n'est pas toujours souhaitable (un mot complexe que tout le monde connaît peut faire gagner du temps).

Le respect des droits de l'homme devrait pousser les juristes à faire plus d'efforts, même si la rédaction parfaite, comme l'homme parfait, n'existe pas. Je sais bien que la rédaction qui suit des négociations peut avoir à être expressément vague ou ambiguë. Je parle de la couche qui ne sert à rien sauf à protéger la culture juridique.

Nous avons besoin donc que les rédacteurs juristes identifient ce qui est superflu vis-à-vis de la loi.

Il reste du travail à faire - beaucoup de travail ! Car il n'a pas vraiment commencé en Europe, où on reste coincé par un conflit académique entre linguistes et juristes à propos des mots...

\footnotetext{
Portalis, Discours préliminaire du premier projet de Code civil (1801)

8 Décision $n^{\circ}$ 2001-455 DC du 12 janvier 2002.
} 



\section{Chapitre 2}

L'ORIGINALITÉ DU « MIEUX LÉGIFÉRER 》 AU REGARD DES EXPÉRIENCES INTERNATIONALES ET NATIONALES 



\title{
Peut-on « Mieux légiférer » \\ EN DROIT INTERNATIONAL?
}

\author{
Romain LE BEUF ${ }^{1}$
}

\section{Résumé}

Les objectifs qui animent le projet «Mieux légiférer » de l'Union européenne ne sont pas inconnus du droit international. Au contraire, de nombreux efforts ont été menés par les États et les organisations internationales dans le but d'améliorer les modes de création du droit international et d'assurer à la fois une meilleure efficacité et une plus grande effectivité à ses dispositions. Cependant, certains caractères propres à la discipline font obstacle à une réception du projet tel qu'il est aujourd'hui conçu par les institutions européennes. La présente contribution a pour objet d'identifier ces difficultés et de s'interroger sur la manière dont l'Union - en tant qu'association d'États peut parvenir à les surmonter.

\section{Abstract}

The "Better Law Making" agreement reached by the European Union in 2016 relies on some principles and aims which are not unknown in international law. States and international organisations have conducted strong efforts to improve normative processes and to ensure a better efficiency to international rules. Some characteristic inherent to the international law nevertheless restrain the ability of this European project to be fully endorsed. This article focuses on those constraints and on the way the European Union can handle them.

«La régénération du droit international n'est possible que suivant les données historiques. En proposant des réformes, il faudra se garder de toute illusion, il faudra se laisser guider par la réalité et par la consultation du passé. Les illusions peuvent charmer, mais elles conduisent souvent à la déception cruelle de leurs apôtres dévoués. »

\footnotetext{
Professeur de droit public, Aix Marseille Univ, Université de Toulon, Univ Pau \& Pays Adour, CNRS, DICE, CERIC, Aix-en-Provence, France.
} «La crise du droit international », RGDIP, 1919, p. 91. 
Les aspirations àmieux légiférerne sont assurément pas spécifiques au droit del'Union européenne. La préoccupation est ancienne et partagée par les divers ordres juridiques. Les termes peu amènes du Président du Conseil constitutionnel, dans son discours de clôture d'un colloque consacré en 2014 à cette question en droit français, témoignent même d'une forme de lassitude par rapport à cet enjeu : il en irait de ces questionnements « comme des saisons ou des fleurs. Ils reviennent avec régularité ${ }^{3}$. Il est vrai que la doctrine française s'est fortement intéressée, ces dernières années, au thème de la «qualité de la loi » $»^{4}$ Le choix du Conseil d'État de consacrer son étude annuelle de 2016 à la simplification et à la qualité du droit témoigne encore du caractère toujours pressant de ce sujet ${ }^{5}$. Par contraste avec ce double engouement des droits internes et du droit européen, on peut avoir le sentiment que la doctrine internationaliste s'est peu souciée de ces enjeux. Pourtant, l'idée de transposer au droit international les problématiques du «Mieux légiférer» paraît s'imposer d'évidence tant le droit international se voit reprocher - plus que tout autre peut-être - un fonctionnement archaïque, lent, peu apte à parvenir à des décisions et moins encore à en assurer la mise en ouvre. Pour le dire de façon abrupte, le droit international constitue sans doute, à bien des égards, l'antithèse du «Mieux légiférer » tel qu'il est promu par les institutions de l'Union européenne. En réalité, la désaffection de la doctrine par rapport à ces questions n'est qu'apparente. Les internationalistes se sont de longue date intéressés à des problématiques qui recoupent pour une très large part les objectifs du «Mieux légiférer ». Les travaux portant sur les modes de création du droit et ceux touchant à l'effectivité des règles adoptées sont légion. Les différences qui opposent de ce point de vue les études menées en droit international à celles conduites en droits internes et européen tiennent moins à l'existence des questions qu'à la nature et à la portée des réponses qui y sont apportées. L'idée de réforme est, il faut l'admettre, plutôt étrangère à la pensée internationaliste ${ }^{6}$. Cette réticence résulte sans doute, pour une part, des propensions conservatrices de la doctrine - pour ne pas dire d'une forme d'aveuglement face au changement ${ }^{7}$. Cependant, et de façon plus fondamentale, cette posture prudente de la doctrine résulte également d'un certain nombre de contraintes structurelles qui rendent en réalité l'idée même d'une transformation significative de la matière difficilement envisageable. Le processus évolutif - sans être absent, tant s'en faut - est à la fois plus diffus et plus «progressif $»^{8}$.

3 Mieux légiférer, Colloque organisé à l'initiative du Président de l'Assemblée nationale, tenu à l'Hôtel de Lassay le 28 novembre 2014 , actes publiés en supplément à $\mathcal{F} C P$ G., 2015, $\mathrm{n}^{\circ}$ 14. L'auteur poursuit en soulignant que si cette régularité « est heureuse s'il s'agit de voir réapparaître les bourgeons; elle est plus lassante quand il s'agit de réfléchir à la médiocrité de la loi. Au sentiment de déjà-vu, s'ajoute celui qu'on peut difficilement remédier à la situation ».

4 V. p. ex. dans la période récente : P. Albertini (éd.), La qualité de la loi : expériences française et européenne - actes du colloque organisé par le CUREf à la Faculté de droit, sciences économiques et gestion de l'université de Rouen les 13 et 14 novembre 2014, Mare \& Martin, Paris, 2015, 312 p.; P. Rrapi, L'accessibilité et l'intelligibilité de la loi en droit constitutionnel - Étude du discours sur la «qualité de la loi», Paris, Dalloz, Nouvelle bibliothèque de thèse, $\mathrm{n}^{\circ}$ 137, 2014, 280 p.; V. aussi les actes du colloque organisé en mai 2013 par l'Institut d'études politiques de Paris sur le thème de «La qualité de la loi », Revue juridique Thémis, n 48-1, 2014, p. 185-277.

5 Conseil d'État, Étude annuelle 2016 - Simplification et qualité du droit, coll. Les rapports du Conseil d'État, La documentation française, Paris, $256 \mathrm{p}$.

6 V. en ce sens M. Koskenniemi, «Repetition as Reform : Georges ABi-SAAB Cours général de droit international public », EfIL, vol. 9, 1998, p. 405-411. L'idée de changement n'est néanmoins pas entièrement absente : v. en ce sens et à différentes périodes A. PELLET, «L'adaptation du droit international aux besoins changeants de la société internationale - conférence inaugurale de la session de droit international public », RCADI, t. 329, 2007, p. 9-48; Ph. CAHIER, «Changements et continuité du droit international : cours général de droit international public », $R C A D I$, t. 195, 1985 ; J. KunZ, «La crise et les transformations du droit des gens », RCADI, t. 88, 1955, p. 1-104.

7 Pour un aveu en ce sens, v. A. PELLET, «L'adaptation du droit international aux besoins changeants de la société internationale conférence inaugurale de la session de droit international public », RCADI, t. 329, 2007, p. 17-18.

8 Cette idée de « développement progressif du droit international» figure expressément dans la Charte des Nations, parmi les missions dévolues à l'Assemblée générale (art. 13, par. 1, lit. a.). 
Ces contraintes particulières imposent au renouvellement du droit international un cheminement qui lui est propre et qui rend délicate la réception en droit international des trop explicites débats nationaux et européens relatifs au «Mieux légiférer». Assurément, cette divergence de méthodes n'exclut pas l'existence, au fond, d'un certain nombre de préoccupations communes. Il ne faut, d'ailleurs, pas méconnaître les démarches entreprises au niveau international pour améliorer les conditions de production et d'application du droit, à la lumière de certains objectifs qui s'apparentent à ceux du «Mieux légiférer» (1). Cependant, ces efforts ne peuvent que se heurter à un ensemble d'obstacles inhérents à la structure de l'ordre juridique international (2).

\section{1) Les efforts internationaux en faveur du «Mieux légiférer»}

La question du « Mieux légiférer » peut être envisagée à un double point de vue en droit international. D'un côté, on peut envisager le droit international comme un moyen pour les États de mieux légiférer dans leurs droits internes respectifs. De l'autre, il faut apprécier, de façon plus spécifique, les efforts menés par les acteurs internationaux en vue d'améliorer les conditions de fonctionnement du droit international lui-même. Il convient donc de distinguer les tentatives de mieux légiférer par le droit international (1.1) de celles destinées à mieux légiférer en droit international (1.2).

\subsection{Les tentatives de mieux légiférer par le droit international}

Le développement des rapports internationaux a sans doute, par lui-même, profondément contribué aux réflexions des États en matière de légistique. Les rapports de coopération et de concurrence qui s'établissent entre des sociétés fondées sur des traditions juridiques différentes engagent nécessairement les États dans une démarche de comparaison de leurs dispositifs législatifs respectifs. De ce point de vue, l'internationalisation des rapports sociaux engendre par elle-même - de façon presque mécanique - une réflexion qualitative sur le droit, réflexion qui est au fondement de toute démarche tendant à mieux légiférer. Le droit international sert ainsi classiquement de moyen permettant d'harmoniser, à partir des expériences et des difficultés rencontrées par chaque État, le contenu des normes internes. De façon plus occasionnelle, le droit international détermine également, au-delà du seul contenu des normes internes, les procédures en vertu desquelles elles doivent être produites.

\section{L'harmonisation du contenu des normes internes}

Il n'y a pas lieu d'insister sur la fonction d'harmonisation des droits internes par le droit international. Sans aller jusqu'à admettre que cette fonction est inhérente au droit international - elle n’a longtemps été que très marginale -, elle est assurément devenue centrale avec la multiplication des conventions multilatérales sectorielles destinées à coordonner les politiques nationales dans différents domaines à partir de la seconde moitié du XIX ${ }^{\mathrm{e}}$ siècle. Cette harmonisation peut résulter de considérations distinctes. 
Dans la majeure partie des cas, il s'agit pour les États de prévenir ou de réagir au développement de certains comportements transnationaux que les droits internes ne sont pas, à eux seuls, en mesure de réguler. Le passage par le droit international est alors un moyen pour les États d'assurer l'adaptation de dispositifs nationaux condamnés, en l'absence de coordination internationale, à l'ineffectivité. Ce souci d'efficacité - parfois expressément mentionné dans le préambule des accords ${ }^{9}$ - permet alors de rattacher ce procédé à l'une des problématiques cardinales du « Mieux légiférer ». L'harmonisation des droits nationaux peut également viser - tout à l'inverse, - à encourager certains comportements, notamment en assurant l'accessibilité et l'intelligibilité du droit ${ }^{10}$, le cas échéant par la diminution ou la suppression de particularismes juridiques nationaux ${ }^{11}$. Ce triple souci d'accessibilité, d'intelligibilité et d'encouragement aux activités économiques rencontre, là encore très nettement, les fondamentaux du « Mieux légiférer ».

Dans d'autres cas, le recours au droit international ne vise pas à répondre à des enjeux internationaux par nature, mais à identifier en commun un ensemble de bonnes pratiques permettant de répondre à des problèmes d'ordre purement interne, mais communs à plusieurs États. La détermination des règles internes les plus pertinentes fait alors l'objet de discussions au niveau international, éventuellement dans le cadre de commissions dont les travaux et études vont inspirer les règles qui seront ensuite édictées au niveau national par chaque État. On trouve un exemple de ce procédé dans la Convention de 1946 relative à la suppression de l'habitude de fumer l'opium : cette convention comporte un certain nombre de prescriptions destinées à limiter, sur un plan interne, la consommation de ce stupéfiant : monopole d'État, rationnement, âge minimum, prohibition de la vente à crédit, etc. Selon les termes du préambule de cette convention, ces diverses dispositions étaient établies par les parties contractantes à partir des «conclusions et suggestions de la Commission chargée par la Société des Nations de procéder à une enquête sur le contrôle de l'opium à fumer en ExtrêmeOrient $»^{12}$. De façon plus contemporaine, les multiples enquêtes et études confiées à des organisations internationales, telles que l'OCDE, sont autant de moyens pour les États de se concerter afin d'améliorer l'efficacité de leurs réglementations internes respectives dans divers secteurs, rencontrant ici encore les attentes du « Mieux légiférer».

9 Convention internationale pour la répression de la circulation et du trafic des publications obscènes, conclue à Genève le 12 septembre 1923, RTSDN, vol. 27, p. 214 : les États parties s'affirment «[é]galement désireux de donner le plus d'efficacité possible à la répression de la circulation et du trafic des publications obscènes »

$10 \mathrm{~V}$. en ce sens la remarquable résolution de l'Institut de droit international comportant des Propositions pour un accord international aux fins de l'institution d'un Comité international permanent pour faciliter aux gouvernements et aux citoyens de chaque pays la connaissance des lois actuellement en vigueur (Résolution adoptée le 12 septembre 1885 par l'Institut, à l'occasion de sa session de Bruxelles).

11 C'est le cas, parmi de nombreux exemples, de la convention sur la circulation routière du 19 septembre 1949, dans laquelle les États se disent «désireux de favoriser le développement de la circulation routière internationale [...] en établissant des règles uniformes » (RTNU, vol. 125, p. 31).

12 Accord relatif à la suppression de l'habitude de fumer l'opium, conclu à Bangkok le 27 novembre 1931, RTSDN, vol. 177, 1937, p. 374, préambule. 


\section{L'amélioration des procédures normatives internes}

Plus rarement, le droit international ne va pas seulement avoir pour but d'assurer la qualité substantielle des normes nationales, mais va également tendre à une amélioration des procédures de création et d'application du droit. Les exemples sont longtemps demeurés rares et les préconisations très générales. De façon très évasive, on pourra citer l'article 25 du Pacte international sur les droits civils et politiques, qui tend à imposer la démocratie comme forme de gouvernement, et donc comme mode de production du droit. Tout citoyen doit ainsi avoir «le droit et la possibilité [...] [d]e prendre part à la direction des affaires publiques, soit directement, soit par l'intermédiaire de représentants librement choisis ». L'Organisation des Nations unies s'est prononcée dans le même sens en incorporant dans ses Objectifs du millénaire une rubrique consacrée à la «bonne gouvernance », et qui appelle à «l'adoption dans tous les pays de processus politiques plus égalitaires, qui permettent la participation effective de tous les citoyens à la vie politique $»^{13}$. Ces exigences très générales sont parfois complétées, sur un plan sectoriel, par quelques exigences plus spécifiques. Par exemple, dans le domaine commercial, les accords de Cotonou conditionnent certains avantages commerciaux à la réalisation, par les États bénéficiaires, de «réformes institutionnelles» fondées sur les idées de démocratie, de transparence et de bonne gouvernance ${ }^{14}$. Le développement des obligations internationales relatives à l'exigence préalable de réalisation d'études d'impact en amont de certaines actions étatiques contribue également à améliorer les modalités de prise de décision en droit interne. La Convention des Nations unies sur le droit de la mer prévoit ainsi l'obligation pour les États de procéder à une évaluation a priori des effets de leurs projets ${ }^{15}$ et de communiquer les résultats de cette évaluation à la communauté internationale ${ }^{16}$. Des telles obligations d'évaluation préalable sont aujourd'hui fréquentes en droit de l'environnement ${ }^{17}$.

Ces quelques initiatives au niveau universel rencontrent également un écho au niveau régional. Le Conseil de l'Europe a ainsi multiplié les instruments destinés à encadrer l'exercice du pouvoir politique dans les États membres, et donc en partie à favoriser certaines modalités de création du droit. Sans revenir sur la très classique promotion de la démocratie ${ }^{18}$, le Conseil de l'Europe a explicitement promu, en adoptant dès 1985 une Charte européenne de l'autonomie locale, un modèle de

13 Assemblée générale des Nations unies, Résolution 55/2, 8 septembre 2000, pt. 5.

14 Accord de partenariat entre les membres du groupe des États d'Afrique, des Caraïbes et du Pacifique, d'une part, et la Communauté européenne et ses États membres, d'autre part, signé à Cotonou le 23 juin 2000, JOUE, L. 317, 15 décembre 2000, p. ex. arts 1, 9 par. 3, 20 par. 1 et 33. De façon plus spécifique, on mentionnera également le domaine du rétablissement et de la consolidation de la paix, dans lequel le cadre politique interne est établi, de façon parfois très minutieuse, par un instrument de nature internationale. On se bornera à cet égard à rappeler que le cadre constitutionnel de la Bosnie-Herzégovine est défini par l'Annexe 4 à l'Accord-cadre pour la paix conclu en 1995 à Dayton. Cette annexe prévoit ainsi la répartition des compétences entre le gouvernement fédéral et les entités fédérées, la structure bicamérale de l'Assemblée parlementaire et les procédures applicables à l'adoption des lois, ainsi que la procédure applicable à l'adoption des actes du pouvoir exécutif. 15 Convention des Nations unies sur le droit de la Mer, signée à Montego Bay le 10 décembre 1982, art. 206 : «Lorsque des États ont de sérieuses raisons de penser que des activités envisagées relevant de leur juridiction ou de leur contrôle risquent d'entraîner une pollution importante ou des modifications considérables et nuisibles du milieu marin, ils évaluent, dans la mesure du possible, les effets potentiels de ces activités sur ce milieu».

16 Convention des Nations unies sur le droit de la Mer, précit., art. 205 et 206. Dans sa récente sentence à propos du différend en Mer de Chine méridionale, le Tribunal a considéré que le simple fait pour un État de ne pas publier les résultats d'une étude d'impact environnemental suffit à constituer une violation de l'article 206 (The South China Sea Arbitration (The Republic of Philippines v. The People's Republic of China), sentence du 12 juillet 2016, par. 991).

17 V. sur ces questions M. Prieur, «Instruments internationaux et évaluation environnementale de la biodiversité : enjeux et obstacles », RfE, 2011/5, p. 7-27 et E. NAIM-GeSBERT, «L'étude d'impact écologique », RfE, 2015/4, vol. 40, p. 597-599.

18 V. p. ex. Assemblée parlementaire du Conseil de l'Europe, Résolution 1154 (1998), Fonctionnement démocratique des Parlements nationaux, adoptée le 20 avril 1998. 
gouvernance fondé sur la décentralisation. La Charte énonce ainsi que «l'existence de collectivités locales investies de responsabilités effectives permet une administration à la fois efficace et proche $d u$ citoyen ${ }^{19}$. Ce double souci d'efficacité et d'adéquation des politiques publiques aux besoins des administrés rejoint ici les préoccupations du « Mieux légiférer ».

L'OCDE a de la même façon institutionnalisé un programme portant sur la «coopération réglementaire $»^{20}$. Ce programme s'appuie sur un «Comité de la politique et de la réglementation [ayant] pour mandat d'aider les Membres et non-Membres à établir et renforcer leur capacité à œuvrer pour la qualité et l'adaptation de la réglementation ${ }^{21}$. Le Conseil de l'OCDE a entériné les travaux du Comité en adoptant une recommandation qui, selon les termes du Président du Comité, «adresse aux pouvoirs publics des conseils quant au renforcement des institutions et à l'application d'outils de gestion de la réglementation $»^{22}$. Cette recommandation «propose aussi des outils de mesure ou des points de référence concrets permettant aux pays d'évaluer leur aptitude à élaborer et à appliquer une réglementation de qualité ${ }^{23}$. D'une façon plus générale, la recommandation est destinée à servir «de base à un dialogue plus clair avec les Membres et les non-Membres sur les politiques, les pratiques et les institutions nécessaires à l'amélioration systémique de la qualité de la réglementation. Elle facilitera la mise en place de pratiques comparatives et, partout où les capacités actuelles sont limitées, servira de cadre à la formulation de conseils à l'intention des pays désireux d'améliorer leur gouvernance réglementaire $»^{24}$.

Ces différents cadres, adoptés dans des contextes très divers, traduisent tous l'émergence d'un ensemble de principes internationaux s'appliquant à la création des règles de droit au sein des États. Sans être rigoureusement homogènes, ces principes sont néanmoins convergents dans leurs lignes directrices et rejoignent les principales préoccupations du « Mieux légiférer ».

\subsection{Les tentatives de mieux légiférer en droit international}

Reste à déterminer dans quelles mesures ces préoccupations appliquées aux États demeurent pertinentes pour guider les conditions de l'élaboration du droit international lui-même. Il convient à cet égard de souligner que l'amélioration des conditions de production et de mise en œuvre du droit international est une aspiration constante, même s'il est vrai qu'elle n'a formellement donné lieu qu'à des initiatives limitées.

19 Charte européenne de l'autonomie locale, adoptée le 15 octobre 1985, préambule (nous soulignons). Cet engagement du Conseil de l'Europe en faveur de la décentralisation a connu une nouvelle impulsion, les 15 et 16 octobre 2007, de la part des ministres européens responsables des collectivités locales et régionales par la promotion d'une «Stratégie pour l'innovation et la bonne gouvernance au niveau local», formellement adoptée par le Comité des Ministres le 26 mars 2008. Dans le cadre d'un programme intitulé «Éloges», le Conseil de l'Europe remet également des prix de «bonne gouvernance » aux collectivités locales des États membres respectant les principes énoncés dans la stratégie de 2007 et tient régulièrement des conférences sur les «bonnes pratiques» au niveau des administrations locales, avec le projet de convertir ces pratiques en programmes de formations. Ce programme, s'il s'adresse en premier lieu aux États membres, cherche désormais à s'exporter, comme en témoigne la création du partenariat oriental. Sur ces programmes, v. le site internet du Conseil de l'Europe, sous les rubriques «Démocratie» et «Bonne gouvernance».

20 V. les multiples rubriques dédiées à ce programme sur le site de l'OCDE [www.oecd.org/fr/gov/politique-reglementaire; lien valide au 7 avril 2017] OCDE, Recommandation du Conseil concernant la politique et la gouvernance réglementaires, 2012, p. ii.

G. BANKs, «Avant-propos», in Recommandation du Conseil concernant la politique et la gouvernance réglementaires, précit., p. 2.

Ibid.

Ibid. 


\section{Une aspiration constante}

Les problématiques du « Mieux légiférer » ne font certainement pas défaut en droit international. Les auteurs qui se sont intéressés à la matière s'en sont saisis de longue date. Dans son Projet de paix perpétuelle, Kant laisse déjà entrevoir des considérations relatives à la publicité des traités internationaux, estimant qu' « $[\mathrm{u}] \mathrm{n}$ article secret dans les traités de droit public est objectivement, c'est-à-dire relativement à son contenu, une contradiction $»^{25}$. La doctrine juridique lui emboîtera d'ailleurs le pas à la fin du XIX ${ }^{\mathrm{e}}$ siècle, l'Institut de droit international formant notamment dès 1885 un «Vœu concernant la publication des traités ${ }^{26}$ et proposant quelques années plus tard, en 1891 , la constitution d'une «Union pour la publication des traités ${ }^{27}$ qui assurerait « une publication aussi universelle, aussi prompte et aussi uniforme que possible, des traités et conventions entre les États $»^{28}$. Il s'agit là de l'une des premières manifestations positives du souci de produire un droit international, sinon de meilleure qualité, du moins plus transparent et plus accessible. Cette exigence de publicité sera très vite incorporée au Pacte de la Société des Nations ${ }^{29}$ et figure toujours, aujourd'hui, à l'article 102 de la Charte des Nations unies ${ }^{30}$.

Dans un même ordre d'idées, l'énumération des sources du droit international dans le Statut de la Cour permanente de Justice internationale, aujourd'hui l'article $38 \mathrm{du}$ Statut de la Cour internationale de Justice, peut être considérée comme une importante contribution à la prévisibilité du droit international. Les nombreux projets de codification du droit international coutumier témoignent encore de la volonté de substituer à l'incertitude d'une source obscure des principes à la fois accessibles et intelligibles ${ }^{31}$. Très spécifiquement, les travaux de la Commission du droit international relatifs à la codification du droit des traités ${ }^{32}$, aux actes unilatéraux des États ${ }^{33}$ ou à l'identification de la

25 KANT, «Essai philosophique sur la paix perpétuelle - deuxième supplément» in Éléments métaphysiques de la doctrine du droit, trad. Jules BARNi, éd. Auguste Durand, Paris, p. 316.

26 Institut de droit international, Vou pour la publication des traités, résolution adoptée le 11 septembre 1885 à l'occasion de la session de Bruxelles.

27 Institut de droit international, Voeu concernant la formation d'une Union internationale pour la publication des traités, résolution adoptée le 12 septembre 1891 à l'occasion de la session de Hambourg. L'Institut adoptera dès l'année suivante un projet de convention concernant la création de cette Union (projet adopté le 7 septembre 1892 à l'occasion de la session de Genève). Sur cette question, v. M. Rostworowski, «L'Union internationale pour la publication des traités », RGDIP, 1894, p. 135 et s.

$28 \quad$ Ibid.

29 Pacte de la Société des Nations, article 18: «Tout traité ou engagement international conclu à l'avenir par un membre de la Société devra être immédiatement enregistré par le secrétariat et publié par lui aussitôt que possible. Aucun de ces traités ou engagements internationaux ne sera obligatoire avant d'avoir été enregistré ».

30 Charte des Nations unies, art. 102 «1. Tout traité ou accord international conclu par un Membre des Nations unies après l'entrée en vigueur de la présente Charte sera, le plus tôt possible, enregistré au Secrétariat et publié par lui. 2. Aucune partie à un traité ou accord international qui n'aura pas été enregistré conformément aux dispositions du paragraphe 1 du présent Article ne pourra invoquer ledit traité ou accord devant un organe de l'Organisation ».

31 Ce souci de codifier le droit international est ancien, ainsi qu'en témoigne déjà le manuel de M. BLunTSCHLI, Le droit international codifié, trad. Par M. C. LARDY, éd. Guillaumin, Paris, 1874. Mentionnant déjà, quelques années auparavant, l'idée suggérée par Jeremy BENTHAM d'un «universal international code» («Principles of International Law » in The Works of feremy Bentham», W. TAIT, Édimbourg, 1843, vol. II, p. 537). Pour une présentation générale des efforts de codification antérieurs à la création de la Commission du droit international en 1947 , v. La Commission du droit international et son ouvre, Nations unies, New York, 2009, vol. I, spéc. p. 1-8.

32 La Commission s'est attachée à de nombreux aspects du régime des traités internationaux, qu'il s'agisse des traités entre États (le projet d'articles adopté en 1966 a donné naissance à la Convention de Vienne de 1969 sur le droit des traités entre États) ou conclus par des organisations internationales (le projet adopté en 1982 donnera lieu à une convention en 1986). D'autres travaux portent sur des aspects plus spécifiques (réserves aux traités, 2011; effets des conflits armées, 2011), certains sujets étant toujours en cours (les accords et la pratique subséquentes en relation avec l'interprétation des traités, depuis 2008; l'application provisoire des traités, depuis 2012).

33 La Commission a adopté en 2006 une série de Principes directeurs applicables aux déclarations unilatérales des États susceptibles de créer des obligations juridiques (Ann. CDI, 2006, vol. II (2)). 
coutume $^{34}$ sont autant de démarches tendant à une rationalisation des modes de production des normes en droit international public ${ }^{35}$. D'une façon plus indirecte, les efforts de «la doctrine des publicistes les plus qualifiés des différentes nations ${ }^{36}$ tendent eux aussi à assurer une plus grande cohérence à ce «bric-à-brac » normatif qu'a longtemps pu être le droit international ${ }^{37}$. Il existe donc sans aucun doute une véritable aspiration à mieux légiférer en droit international.

\section{Des initiatives limitées}

Cependant, les conséquences pratiques de cette aspiration à produire un droit international de meilleure qualité demeurent limitées. Ce n'est pas que les efforts font défaut.

Les organisations internationales s'attachent, chacune en ce qui la concerne, à améliorer les conditions de leur action. Si les bases de données informatisées des Nations unies ne renvoient pour l'heure aucune réponse en ce qui concerne l'expression «Mieux légiférer», la substance de cette préoccupation transparaît régulièrement dans les travaux de l'Organisation. Dès 1985, l'Assemblée générale des Nations unies adopte une résolution intitulée «Examen de l'efficacité du fonctionnement administratif et financier des Nations unies $»^{38}$, question qui restera inscrite de façon permanente à son ordre du jour sous des intitulés qui glisseront peu à peu, au fur et à mesure des résolutions, vers le souci de «revitalisation des travaux» de l'Assemblée ${ }^{39}$. Les objectifs que se fixe l'Assemblée recoupent assez largement ceux établis par l'Union européenne dans le cadre de son programme «Mieux légiférer ». La résolution 58/126 du 19 décembre 2003 - l'une des plus complètes et des plus ambitieuses en la matière - énonce ainsi la nécessité de «rationaliser davantage les méthodes de travail de l'Assemblée générale, afin qu'elle fonctionne avec plus d'efficience et d'efficacité $»^{40}$, en adoptant des «résolutions [...] plus concises, mieux centrées et à finalité plus concrète ${ }^{41}$ et en faisant en sorte que «les travaux et les décisions de l'Assemblée soient mieux diffusés ${ }^{42}$. Plus généralement, l'Assemblée s'engage dans un processus d'évaluation devant lui permettre « de déterminer les pratiques optimales et les meilleures méthodes de travail $»^{43}$, tout en se donnant les moyens lui permettant d'assurer «l'application effective des résolutions adoptées $»^{44}$. Toutefois, le projet s'enlise assez vite dans les contradictions et l'Assemblée - tout en affirmant son souhait de concision et d'efficacité -

\footnotetext{
34 La Commission a inscrit en 2012 à son ordre du jour la question de l'Identification du droit international coutumier. Noter que l'article 24 du Statut de la Commission du droit international lui donne pour mission d' «examin[er] les moyens susceptibles de rendre plus accessible la documentation relative au droit international coutumier, par exemple la compilation et la publication de documents établissant la pratique des États et des décisions de juridiction nationales et internationales sur des questions de droit international, et [de faire] rapport à l'Assemblée générale sur ce sujet ». La Commission a remis son rapport à l'Assemblée générale dès 1950 (Ann. CDI, 1950, vol. II, p. 367 et s.).

35 Sur certaines analogies méthodologiques de la codification et du Mieux légiférer, v. infra, Partie 2.

36 Pour reprendre les termes de l'article 38 de la Cour internationale de Justice.

J. CombACAU, «Le droit international : bric-à-brac ou système? », APD, 1986, vol. 31, p. 85-105.

Assemblée générale des Nations unies, Résolution 40/237, 18 décembre 1985.

Assemblée générale des Nations unies, Résolution 70/305, 13 septembre 2016. V. précédemment la série des résolutions 41/213, 48/264,

52/12 B, 58/126, 58/316, 59/313, 60/286, 63/309, 67/297, 68/307 et 69/321.

Assemblée générale des Nations unies, Résolution 58/126, 13 janvier 2004, part. B.

Ibid., part. B, pt. 6.

Ibid., part. A, pt. 8.

Ibid., part. B, pt. 8 .

Assemblée générale des Nations unies, Résolution 59/313, 12 septembre 2015, pt. 1.
} 
entasse les rapports sur la question ${ }^{45}$, multiplie les résolutions ${ }^{46}$ et souligne la nécessité «d'appliquer les résolutions relatives à la revitalisation de ses travaux ${ }^{47}$; celles-là mêmes qui avaient pour objet, précisément, d'assurer l'effectivité des décisions de l'organe. Les résultats pratiques des efforts consentis en vue d'améliorer la qualité de la norme au niveau de l'Organisation des Nations unies demeurent donc assez faibles ${ }^{48}$.

Cependant, il faut ici encore faire état des travaux récemment entrepris dans le cadre de l'OCDE dans le domaine de la coopération réglementaire. L'OCDE a ainsi initié une large étude des modes de production du droit au sein des organisations internationales elles-mêmes. Il en est résulté un rapport, publié en 2016, intitulé International Regulatory Co-operation - The Role of International Organisations in Fostering Better Rules of Globalisation ${ }^{49}$. Le rapport, établi à partir de l'analyse des travaux d'une cinquantaine d'organisations internationales, souligne d'emblée « the diversity of international organisations involved in standard-setting and rule making $»^{50}$, tout en mettant en exergue le caractère commun des objectifs de cet «international rule making ${ }^{51}$ tourné vers l' «inclusivité », la «transparence », la «pertinence», l' «efficience» et la «flexibilité ${ }^{52}$. S'il est trop tôt pour tirer un bilan pratique de cette coopération réglementaire, il est néanmoins très net que le lexique employé recoupe l'essentiel des objectifs du « Mieux légiférer » promu par l'Union. Il existe donc un important travail réalisé à la fois au sein et entre les différentes organisations internationales, en vue d'améliorer la qualité des normes produites sur le plan international.

Pour autant, il importe de souligner que le produit de ces efforts demeure limité. Les démarches initiées par les organisations internationales sont entravées par des obstacles qui échappent, en réalité, à leur bonne volonté. De plus, il faut noter que les travaux de ces organisations demeurent confinés en leur sein et ne peuvent constituer des leviers efficaces en ce qui concerne les procédures normatives extérieures à ces organisations. Dans un cas comme dans l'autre, les États restent les maîtres dans l'élaboration du droit international. Or, leur activité diplomatique reste souvent déterminée - au moins en partie - par des considérations liées à leurs ordres juridiques nationaux, sinon à leurs stratégies de politique extérieure ${ }^{53}$. La légistique internationale est donc assez largement prédéterminée par des considérations qui échappent à toute tentative de rationalisation. Il existe donc des obstacles structurels à la réception du « Mieux légiférer » dans l'ordre international.

\footnotetext{
45 V. de façon significative la Résolution 63/309 du 14 septembre 2009 : l'Assemblée y « [p] rend note avec satisfaction du rapport du Groupe de travail spécial sur la revitalisation de l'Assemblée générale» (pt. 1) et, dès le paragraphe suivant, «[d]écide de créer [...] un groupe de travail spécial sur la revitalisation de l'Assemblée générale » chargé de lui «présenter un rapport à ce sujet» dès la prochaine session (pt. 2).

46 V. le catalogue dressé par l'Assemblée dans sa résolution 63/309: «Réaffirmant ses résolutions relatives à la revitalisation de ses travaux, notamment les résolutions 46/77 du 12 décembre 1991, 47/233 du 17 août 1993, 48/264 du 29 juillet 1994, 51/241 du 31 juillet 1997, 52/163 du 15 décembre 1997, 55/14 du 3 novembre 2000, 55/285 du 7 septembre 2001, 56/509 du 8 juillet 2002, 57/300 du 20 décembre 2002, 57/301 du 13 mars 2003, 58/126 du 19 décembre 2003, 58/316 du 1er juillet 2004, 59/313 du 12 septembre 2005, 60/286 du 8 septembre 2006, 61/292 du 2 août 2007 et $62 / 276 \mathrm{du} 15$ septembre 2008 ». Les résolutions les plus récentes se bornent à «Réaffirm[er] [...] toutes ses résolutions antérieures », énumérées en note de bas de page.

47 Assemblée générale des Nations unies, Résolution 63/309, précit., préambule.

48 Pour ce qui concerne les efforts de l'Organisation en ce qui concerne les procédures de conclusion des traités internationaux conclus sous ses auspices, v. infra, partie 2.

49 OCDE, International Regulatory Co-operation - The Role of International Organisations in Fostering Better Rules of Globalisation, OCDE Publishing, Paris, 2016, 220 p.

$50 \quad$ Ibid., p. 3.

$51 \quad$ Ibid.

$52 \quad$ Ibid. (nous traduisons).

53 G. DE LAChARrière, La politique juridique extérieure, Economica, Paris, 1983, p. 5.
} 


\section{2) Les obstacles internationaux au «Mieux légiférer»}

L'objectif consistant à transposer le « Mieux légiférer » en droit international se heurte à plusieurs obstacles, qui tiennent simultanément à l'idée de «mieux» et à celle de «légiférer». Saisis dans le contexte du droit international, ces termes présentent une physionomie particulière liée, d'un côté, à la nature spécifique des sources du droit international (2.1) et, de l'autre côté, à l'ambiguïté axiologique inhérente à la discipline (2.2).

\subsection{Les obstacles tenant à la nature des sources du droit international}

La nature des sources du droit international fait doublement obstacle au projet consistant à «Mieux légiférer ». En premier lieu, il faut souligner que le droit international ne comporte pas de procédure permettant de produire des normes susceptibles d'être assimilées - même imparfaitement - à des normes légiférées. En second lieu, il faut insister sur le caractère très décentralisé de la matière, qui permet difficilement d'envisager une refonte d'ensemble en vue de rationaliser et de mettre en cohérence ses divers modes de création du droit.

\section{L'absence de procédure permettant de légiférer en droit international}

Le second des termes compris dans le syntagme «Mieux légiférer» renvoie à une terminologie qui est tout sauf neutre ${ }^{54}$. En effet, et indépendamment de l'idée de progrès suggérée par le comparatif «mieux», l'emploi du verbe «légiférer» est déjà par lui-même porteur d'une certaine exigence qualitative. En effet, dire d'une norme qu'elle est une «norme légiférée », c'est en soi lui reconnaître un ensemble de propriétés - internes et externes - qui la distingue de normes résultant d'autres modes de création du droit. Car légiférer, ce n'est pas réglementer, ce n'est pas convenir, ce n'est pas constater; bref, légiférer, ce n'est pas produire n'importe quel type de norme juridique. Légiférer, à en croire les dictionnaires, c'est « établir des lois», donc des normes de nature législative. Il ne s'agit pas, par cette observation, de s'engager dans une impossible définition de la loi et de ses propriétés intrinsèques. Il importe néanmoins de souligner que l'idée même d'un droit international permettant de «légiférer » soulève d'importantes interrogations quant à la nature de ce droit et quant à son rapport avec ses sujets. Pour prendre la mesure de la difficulté, il faut se souvenir ici du très classique débat - initié par John Austin ${ }^{55}$ - relatif à l'aptitude au prétendu «droit international » à produire des normes juridiques, quelles qu'elles soient ${ }^{56}$. Sans souscrire aux nombreux excès de l'argument, il est vrai que l'on est en droit d'être sceptiques quant à l'existence en droit international

\footnotetext{
54 L'utilisation du terme «légiférer» n'est pas seulement un accident de traduction. Les travaux menés par l'Union européenne oscillent régulièrement entre l'usage des expressions «Améliorer la réglementation» (en anglais : «Better regulation») et «Mieux légiférer» (en anglais : «Better Law Making»). Or, c'est bien cette dernière expression qui apparaît dans l'intitulé du récent accord interinstitutionnel conclu entre le Parlement européen, le Conseil de l'Union européenne et la Commission le 12 mai 2016. L’idée de législation se retrouve également dans les autres versions linguistiques du texte.

55 J. Austin, The Province of Furisprudence Determined, ed. J. Murray, Londres, 1832, spéc. p. 147-148: « rules of this species, which are imposed by opinions current amongst nations, are usually styled the law of nations or international law. Now a law set or imposed by general opinion is a law improperly so called. It is styled a law or rule by an analogical extension of the term » (les italiques sont dans le texte).

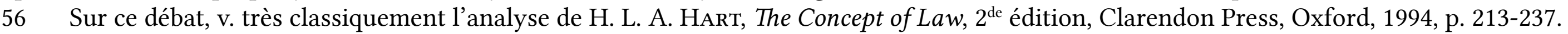


de normes méritant l'appellation de «lois», et donc d'une activité consistant à «légiférer». Cette carence, Michel Virally l'a souligné, ne tient pas à une quelconque infirmité de l'ordre international, mais à ses caractères propres ${ }^{57}$. Tenons-nous en à cette idée, exprimée par la Déclaration des droits de l'homme et du citoyen, de la loi comme «expression de la volonté générale $»^{58}$. Cette condition suffit à elle seule à disqualifier l'essentiel des sources du droit international. En ce qui concerne les sources mentionnées à l'article 38 du Statut de la Cour internationale de Justice, l'incompatibilité est évidente : le traité n'est pas l'expression d'une volonté générale et la coutume, pas plus que les principes généraux, ne consiste en l'expression d'une volonté, quelle qu'elle soit. En effet, il n'y a rien de général dans la technique conventionnelle. Du point de vue de son élaboration, la norme conventionnelle n'est pas, comme le voudrait Jean-Jacques Rousseau, le produit de «plusieurs hommes réunis [qui] se considèrent comme un seul corps [et] n'ont qu'une seule volonté ${ }^{59}$. La négociation diplomatique s'apparente beaucoup plus à ce que le philosophe tient pour l'antithèse du procédé législatif, c'est-à-dire à une synthèse d'«intérêts embrouillés, contradictoires avec le bien commun $»^{60}$. Pour le dire autrement, le traité n'est pas l'expression d'une volonté collective, mais le produit d'un simple faisceau de volontés individuelles concordantes. Le défaut de généralité de la technique conventionnelle se manifeste encore, passée la conclusion du traité, au stade de son application. Étroitement enserré dans les limites de l'adage res inter alios acta, codifié à l'article 34 de la Convention de Vienne, le traité ne lie que les parties sans produire aucun effet sur les tiers. La technique conventionnelle s'avère donc doublement incapable de sublimer la juxtaposition des volontés individuelles en une volonté générale. Elle ne saurait donc être une source permettant de légiférer en droit international.

Pour des motifs différents, la même conclusion s'impose en ce qui concerne la coutume et les principes généraux, qui ne traduisent de la part des États ni une «expression», ni une «volonté». Sous toutes les réserves qu'imposent évidemment les théories réalistes de l'interprétation, coutumes et principes généraux reposent sur un acte de connaissance, et non sur un acte de volonté. Les sources mentionnées à l'article $38 \mathrm{du}$ Statut de la Cour internationale de Justice, si elles permettent de produire des normes, ne permettent donc pas de légiférer. Si l'on s'extrait du cadre sans doute un peu archaïque de cet article, on découvre dans les actes unilatéraux des organisations internationales une source qui se prête à première vue beaucoup mieux à l'idée de «norme légiférée ». En effet, les actes des organisations internationales sont assurément des manifestations de volonté. Cette volonté revêt de plus un indubitable caractère général tant du point de vue de l'élaboration de l'acte que du point de vue de sa portée. En ce qui concerne leur mode de création, les actes unilatéraux sont imputés à l'organisation en tant que telle, et non à chacun de ses membres considérés individuellement. En ce qui concerne leur portée, les actes adoptés vaudront aussi bien pour les États ayant voté en faveur du texte que pour ceux qui s’y sont opposés. Il semble donc bien y avoir, cette fois,

57 M. Virally, «Sur la prétendue "primitivité" du droit international» in Le droit international en devenir - Essais écrits au fil des ans, Puf, Paris, 1990, spéc. p. 98.

58 Déclaration des droits de l'homme et du citoyen, art. 6.

59 J.-J. Rousseau, «Du contrat social, ou principes du droit politique », M.-M. Rey, Paris, 1762, p. 179.

$60 \quad$ Ibid., p. 180. 
sublimation des volontés individuelles, non seulement en volonté collective, mais aussi en volonté générale (étant admis que l'acte ne vaut qu'à l'égard des États membres et que la généralité n'a donc qu'un caractère relatif). Cependant, cette conclusion est toute théorique, car elle fait l'économie de l'hétérogénéité des actes unilatéraux. Or, une approche plus précise conduit à remettre en cause le caractère «législatif» de l'immense majorité des actes adoptés par les organisations internationales. En effet, de très nombreux actes adoptés par les organisations internationales n'ont en réalité qu'une portée réduite à quelques destinataires. Ces actes ont alors le caractère de «décisions » nominatives et circonstancielles, mais ne peuvent prétendre à la qualité de «règles » générales et impersonnelles. Ainsi, notamment, de la majeure partie des résolutions du Conseil de sécurité des Nations unies, dont le pouvoir de décision est borné à l'examen d'une situation d'espèce ${ }^{61}$. À l'inverse, lorsqu'un organe international se voit accorder le pouvoir d'adopter des textes ayant une portée indiscutablement générale, ces textes sont dans l'immense majorité des cas dépourvus de toute normativité et ne revêtent plus le caractère contraignant qui s'attache classiquement à la loi. C'est le cas, toujours aux Nations unies, des résolutions de l'Assemblée générale : ces résolutions constituent pour les États membres de simples recommandations, dépourvues de caractère obligatoire ${ }^{62}$. Les actes unilatéraux des organisations internationales sont donc en pratique enfermés dans cette permanente oscillation entre le défaut de généralité et le défaut de normativité.

Cette oscillation n'est évidemment pas fortuite : elle traduit précisément la réticence des États à constituer un véritable pouvoir législatif au niveau international. Il est ainsi rarissime que les États s'entendent pour réunir entre les mains d'une instance corporative le pouvoir d'adopter des normes obligatoires de portée générale. Même s'il y a d'autres précédents, l'Union européenne demeure une singularité si l'on croise l'étendue de ses compétences à l'autorité normative de ses actes. Et l'on sait encore les réticences à revêtir ces actes du nom de «lois ${ }^{63}$. Le fait est que les États savent sans doute trop bien les enjeux qui s'attachent au pouvoir de légiférer. Ils en ont éprouvé les effets dans leurs ordres internes, et ils ont pu mesurer pleinement la subordination qui en est résultée pour leurs nationaux. On conçoit donc qu'ils soient peu enclins à se soumettre eux-mêmes à une telle prérogative.

\section{L'impossibilité d'une réforme globale des modes de production normative}

La diversité des sources et la pluralité des acteurs du droit international font ressortir une autre difficulté de la réception du projet consistant à « Mieux légiférer » en droit international. En effet, si l'idée d'une réforme destinée à mieux légiférer peut être envisagée dans le cadre restreint et relativement centralisé de l'Union européenne, elle paraît peu transposable à un ordre juridique

61 Les rares fois où le Conseil de sécurité s'est engagé dans l'édiction d'obligations de portée véritablement générale - en matière de terrorisme notamment -, ses résolutions furent bien qualifiées de «législatives» par la doctrine (V. notamment la très explicite qualification de ce pouvoir par P. KLEIN, «Le Conseil de sécurité et la lutte contre le terrorisme : dans l'exercice de pouvoirs toujours plus grands », Revue québécoise de droit international, hors série, 2007, p. 138). Ces décisions furent surtout très fermement dénoncées comme ayant été adoptées ultra vires (V. notamment L. Condorelli, «Le pouvoir législatif du Conseil de sécurité des Nations unies vu à la "loupe Salmon” » in Droit du pouvoir, pouvoir du droit - mélanges offerts à Jean SALMON, Bruylant, Bruxelles, 2007, p. 1229-1239).

62 Charte des Nations unies, arts. 10 à 14.

63 On se souvient que le projet de traité établissant une constitution pour l'Europe avait consacré, en remplacement des classiques «règlements» et «directives», des «lois» et des «lois-cadres» européennes (art. I-33). Ce changement de terminologie n’a pas survécu au traité de Lisbonne. 
international à la fois beaucoup plus étendu, beaucoup plus diffus et beaucoup moins homogène. Il s'agit ici de prendre acte aussi bien de la pluralité des régimes composant le droit international que de leur caractère supplétif.

La pluralité des régimes internationaux rend illusoire toute initiative qui consisterait à rechercher une amélioration d'ensemble des modes de production du droit international. En ce qui concerne les conventions internationales - et exception faite des quelques grandes conventions multilatérales conclues sous les auspices des Nations unies ${ }^{64}$ - les méthodes de négociations relèvent pour l'essentiel de discussions ad hoc entre les services diplomatiques des États concernés, seulement guidées par leurs traditions respectives et les exigences du moment ${ }^{65}$. Il faut d'ailleurs remarquer que cette phase d'élaboration du texte conventionnel est pudiquement éclipsée par la Convention de Vienne, qui saute du constat des pleins pouvoirs des représentants étatiques ${ }^{66}$ à l'adoption définitive d'un texte déjà établi ${ }^{67}$. La Commission du droit international, en codifiant le droit des traités, a donc renoncé à toute tentative d'harmonisation des procédures de discussion du texte. Le même raisonnement vaut en ce qui concerne les processus décisionnels des organisations internationales : ces processus diffèrent d'une organisation à l'autre, et les évolutions souhaitées ne peuvent s'opérer que par la modification successive d'une infinité de traités constitutifs régissant des organisations si diverses qu'il paraît non seulement peu vraisemblable, mais aussi peu opportun, de prétendre les soumettre à une rationalité commune ${ }^{68}$.

Le caractère essentiellement supplétif du droit international limite quant à lui l'efficacité des dispositifs d'harmonisation les plus ambitieux. En effet, les États demeurent à tout moment libres de prendre leurs distances par rapport aux modèles proposés. L'exemple de la Convention de Vienne de 1969 sur le droit des traités est ici encore significatif. La codification de la procédure conventionnelle se présente aux États beaucoup plus comme une ressource que comme une contrainte. Ses dispositions prévoient souvent de façon expresse la possibilité pour les parties de convenir de mécanismes autres que ceux prévus par le codificateur ${ }^{69}$; celles qui ne prévoient pas une telle faculté ne sont d'ailleurs en réalité pas davantage à l'abri d'un aménagement consensuel.

La structure même du droit international n'autorise donc que des évolutions en pointillés, initiées par certains États ou certaines organisations dans certains contextes. Le «Mieux légiférer»

\footnotetext{
64 Il convient également de relever la démarche entreprise par l'Assemblée générale, à la fin des années 1970, en vue du «Réexamen du processus d'établissement des traités multilatéraux ». Initiée par la résolution 32/49 du 8 décembre 1977, la démarche se proposait d'évaluer l'efficacité et la pertinence des procédures appliquées par l'Organisation des Nations unies dans la formulation des textes multilatéraux, afin d'améliorer ces procédures». Cette évaluation, reconduite par les résolutions 36/112 (10 décembre 1981) et 39/90 (13 décembre 1984), donnera lieu à un rapport du Secrétaire général de l'organisation ainsi qu'à de nombreuses communications des États membres, ainsi que de la Commission du droit international (V. le document de synthèse Review of the Multilateral Treaty-Making Process, publié en 1985 sous la côte ST/ LEG/SER.B/21)

65 Pour un aperçu de ces enjeux, v. A. PlAntey, La négociation internationale au XXIe siècle, CNRS Éditions, Paris, 2002, spéc. p. 256-267 et 641-651.

66 Convention de Vienne sur le droit des traités entre États, 1969, art 7.

67 Ibid., art. 9. L'article 8 envisage quant à lui la question de la confirmation par l'État des actes accomplis par des personnes non habilitées à le représenter.

68 Sur la possibilité et les moyens d'une réforme d'ensemble des institutions internationale, v. le très idéaliste article de B. S. CHIMNI, International Institutions Today - An Imperial Global State in the Making, EfIL, vol. 15, n 1, 2004, spéc. p. 36-37.

69 V. la récurrence dans la Convention de l'expression «à moins que le traité n'en dispose autrement » (p. ex. les articles 20 à 22 , relatifs au régime des réserves)
} 
ne saurait donc avoir en droit international général les mêmes ambitions que celles affichées en droit de l'Union européenne. Les difficultés ne sont d'ailleurs pas seulement d'ordre technique. Sur un plan politique, l'Union peut se prévaloir d'une assez forte cohérence axiologique, de nature à imprimer une orientation aux évolutions envisagées. Or, cette cohérence fait essentiellement défaut en droit international général.

\subsection{Les obstacles tenant à l'ambiguïté axiologique du droit international}

L'idée même de «mieux » légiférer présuppose l'existence d'un ensemble de valeurs partagées, qui permet d'assigner un sens aux transformations des modes de production normative. Il importe alors de souligner combien les tensions qui parcourent la société internationale rendent hypothétique une conception univoque de l'amélioration des techniques juridiques. L'expression «Mieux légiférer » présente alors un risque : celui d'occulter, par une formulation consensuelle, le caractère à la fois idéologique et contingent des solutions préconisées.

\section{L'hypothèse d'un «mieux» univoque}

L'adverbe «mieux» ne pose que peu de difficultés lorsque l'on cherche à l'appréhender dans un cadre axiologique clair. On concevra ainsi généralement assez bien ce que peut recouvrir l'idée de «Mieux légiférer» au sein d'une organisation politique reposant sur des valeurs démocratiques. Tout le discours contemporain de la doctrine française sur la qualité de la loi repose ainsi sur un «idéal législatif $»^{70}$ très majoritairement partagé ${ }^{71}$. L'amélioration de la qualité de la loi passera alors notamment par une meilleure association des citoyens au processus de création de la norme et une meilleure accessibilité du droit existant.

En revanche, l'idée de «mieux» légiférer n'a plus de contenu évident dès lors que l'on essaie de la saisir dans un cadre axiologique ambigu, comme l'est celui du droit international. En effet, il n'existe pas - tant s'en faut - d'aspiration démocratique unanime en droit international. Il ne s'agit pas seulement, par cette remarque, de tracer une ligne entre les États démocratiques et les autres. En réalité, les grandes démocraties elles-mêmes sont les premières garantes de processus normatifs respectueux de leur souveraineté. Les procédés de la diplomatie internationale ne comportent aucune des exigences qui sont celles associées à la démocratie en droit interne : la convention de Vienne, notamment, n'instaure aucune obligation de transparence des négociations, ne prescrit aucune association - même minimale - des parlements nationaux et ne tire pour ainsi dire aucune conséquence d'une violation des règles internes de l'État de droit lors de la conclusion d'un traité ${ }^{72}$.

\footnotetext{
70 V. MARInESE, L'idéal législatif du Conseil constitutionnel - Études sur les qualités de la loi, thèse, Nanterre, 2007, 581 p.

71 Ce qui ne doit certainement pas conduire à occulter le caractère contingent et évolutif de cet idéal. Jean-Marie CARBASSE rappelle à cet égard la mutation qui s'est opérée en France lors de la période révolutionnaire : «[c]'est contre cette chatoyante diversité, héritée d'une longue histoire, que l'esprit philosophique du XvIII ${ }^{\mathrm{e}}$ siècle s'est acharné. La raison se dresse alors contre l'histoire, rejette le multiple, exige l'un et l'uniforme [...]. Du point de vue technique, le nouvel idéal législatif culmine dans l'idée de Code, désormais défini comme législation systématique, nouvelle, exhaustive » (J.-M. CARBAsse, Histoire du droit, Puf, Paris, Que sais-je ?, p. 97-98).

72 V. seulement le très restrictif article $46:$ « 1 . Le fait que le consentement d'un État à être lié par un traité a été exprimé en violation d'une disposition de son droit interne concernant la compétence pour conclure des traités ne peut être invoqué par cet État comme viciant son consentement, à moins que cette violation n'ait été manifeste et ne concerne une règle de son droit interne d'importance fondamentale.
} 
La démocratie - pas plus qu'aucune autre valeur d'ailleurs - ne peut donc servir de compas universel permettant d'attribuer un sens univoque à l'idée de «mieux» légiférer : l'intelligibilité de la norme se heurte aux intérêts divergents des négociateurs et au rôle fondamental de l'ambiguïté - plus ou moins constructive - dans la conclusion des accords internationaux. L'efficience, l'effectivité et l'efficacité sont elles-mêmes suspectes, en ce qu'elles accentuent les contraintes que fait peser le droit international sur la souveraineté des États. Pour autant, on ne saurait davantage élever de façon univoque cette souveraineté au rang de paradigme méthodologique indiscuté dans les conférences internationales. Si les États tendent à privilégier des modes de création et d'application du droit respectueux de leur indépendance et de leur égalité, cette propension est contrebalancée par leur souci d'universaliser certaines valeurs ou certains intérêts, y compris en surmontant les oppositions des États réticents. Cette tension entre souveraineté et universalité est permanente. La Cour internationale de Justice en avait déjà fait un élément structurant du régime des traités internationaux dans son avis de 1951 relatif aux Réserves à la convention pour la prévention et la répression du crime de génocide ${ }^{73}$. La lecture des travaux préparatoires à l'adoption de la Convention de Vienne, en 1969, illustre pleinement la manière dont les propositions formulées par la Commission du droit international ont le plus souvent fait l'objet de propositions d'améliorations contradictoires selon que les États auteurs des amendements étaient guidés par le souci de préservation de leur volonté souveraine ou par celui de promotion des intérêts collectifs. Les discussions ayant précédé l'adoption de l'article 9 de la Convention rendent compte de ces contradictions. Le second paragraphe de cet article se lit ainsi : « [1]'adoption du texte d'un traité lors d'une conférence internationale s'effectue à la majorité des deux tiers des États votants ». Cette disposition, qui permet d'adopter un texte conventionnel contre l'avis d'une minorité d'États, constitue donc une dérogation à la règle de l'unanimité qui prévaut ordinairement dans les rapports interétatiques ${ }^{74}$. Cette dérogation à la règle de l'unanimité peut faire l'objet de deux lectures très différentes selon que l'on se place du point de vue de la souveraineté des États participants ou de l'intérêt commun qui s'attache au succès de la négociation. Le commentaire de cette disposition par la Commission du droit international laisse nettement entrevoir les positions contraires qui se sont exprimées lors des travaux de codification entre les membres soucieux de protéger les États minoritaires et ceux attentifs à la bonne marche des négociations $^{75}$. Ces vues divergentes seront reprises par les États lors de la Conférence de Vienne de 1969. Plusieurs États, parmi lesquels la France, chercheront à modifier le texte proposé par la Commission en réduisant la portée de l'exception ${ }^{76}$. D'autres, comme l'Italie, s'interrogeront plus largement sur

\footnotetext{
2. Une violation est manifeste si elle est objectivement évidente pour tout État se comportant en la matière conformément à la pratique habituelle et de bonne foi ». Sur la genèse et la portée de cet article, v. J. HosterT. «Droit international et droit interne dans la Convention de Vienne sur le droit des traités du 23 mai 1969», AFDI, vol. 15, 1969, spéc. p. 92-121.

73 Réserves à la Convention sur le Génocide, avis consultatif du 28 mai 1951, CIJ Recueil 1951, spéc. p. 21-24.

74 Sur cette hypothèse de l'émergence d'un «phénomène majoritaire » en droit international, v. P.M. Dupuy et Y. KERBRAT, Droit international public, $3^{\mathrm{e}}$ éd., Dalloz, Paris, 2016, p. 408.

75 Commentaire du projet d'articles sur le droit des traités entre États, Ann. CDI, 1966, vol. II, p. 211-212.

76 L'amendement français ne portait pas, il est vrai, sur le principe même de l'adoption majoritaire, mais sur l'exclusion de ce principe pour les traités multilatéraux restreints (V. l'intervention de M. ViRALLY, représentant de la France, lors de la $15^{\mathrm{e}}$ séance de la Commission plénière de la Conférence de Vienne, le 5 avril 1968, in Conférence des Nations unies sur le droit des traités, Première session, Vienne, 26 mars - 24 mai 1968 : documents officiels, comptes rendus analytiques des séances plénières et des séances de la Commission plénière, éd. Nations unies, New York, 1969, p. 87-88, par. 2-3.
} 
la nécessité même de déroger au principe de l'acceptation unanime ${ }^{77}$. Pour ces États, l'amélioration du texte était à rechercher dans une meilleure protection de la volonté propre de chaque partie. À l'autre extrémité, d'autres États, comme la Suisse, jugèrent la majorité qualifiée des deux tiers regrettablement protectrice d'intérêts minoritaires ${ }^{78}$. Pour ces États, l'amélioration de la procédure était à rechercher dans la promotion du principe majoritaire. En définitive, la majorité qualifiée des trois cinquièmes constitue donc un moyen terme entre le principe classique de l'unanimité - respectueux de la souveraineté étatique - et le principe de la simple majorité - favorable à l'extension du droit international. La règle consacrée n'a donc rien d'un idéal : elle fait au contraire figure de compromis chancelant entre deux idéaux opposés. On pourrait conduire le même raisonnement à partir de la question des réserves ou des modalités de vote dans les organisations internationales, le résultat demeurerait le même : la question du «mieux» est indépassable tant qu'il n'existe pas de compas commun permettant de fixer un cap aux améliorations attendues ${ }^{79}$.

De ce point de vue, il semble que le droit de l'Union se ressente encore de ses origines internationales. Certes, les États membres ont fermement établi, dans le préambule des traités constitutifs de l'Union, le cap d'une démocratie allant sans cesse vers une union plus étroite entre les peuples. Toutefois, il est patent que cette affirmation textuelle n'a pas levé l'ambiguïté axiologique sur laquelle est bâtie l'organisation, qui oscille en permanence entre l'autonomie communautaire, la démocratie citoyenne et la souveraineté étatique. La notion de «mieux »se pose alors nécessairement comme une question, puisque les différentes composantes de l'Union n'envisagent pas les améliorations à la lumière des mêmes attentes. Les tractations d'apothicaires lors des discussions relatives à la détermination des règles de la majorité qualifiée ou la question du nombre de commissaires ne sont à cet égard que deux illustrations saillantes de la lutte qui prend place entre les valeurs divergentes qui sont au fondement de cette association démocratique d'États souverains. Cette tension originelle implique là encore de résister à la tentation d'un mieux univoque - qu'il soit démocratique, fédéral ou souverainiste. Cette univocité serait par elle-même la négation de la dynamique du projet européen ${ }^{80}$.

\footnotetext{
77 Intervention de M. MArescA, représentant de l'Italie, in Conférence des Nations unies sur le droit des traités, précit. p. 89, par. 22 : «la règle de l'unanimité est celle qui doit régir l'adoption des traités bilatéraux et [...] elle pourrait également convenir pour les traités conclus entre un grand nombre de parties». Le représentant italien n'accepte en définitive l'article discuté qu'au regard du fait que «la règle de l'unanimité donnerait à chaque partie le droit de veto ».

78 Intervention de M. BindschedLeR, représentant de la Suisse, in Conférence des Nations unies sur le droit des traités, précit., p. 89 , par. 18 : «Étant donné que la règle de la majorité des deux tiers permet à une minorité de paralyser l'adoption d'un traité, M. BINDSCHEDLER est d'avis que la règle de la majorité simple serait plus pratique, mais la communauté internationale n'est manifestement pas prête à adopter une telle règle».

79 V. dans le même sens, à propos du rôle dévolu par les États aux Nations unies dans la création du droit international, M. H. ArsanJANI, The United Nations and International Law-Making, RCADI, t. 362, 2012, spéc. p. 17 et s.

80 L'accord interinstitutionnel 13 avril 2016 traduit d'ailleurs une nette conscience de cette pluralité d'enjeux de la part des institutions de l'Union. Le texte explicite à cet égard l'attachement de chaque institution à la «méthode communautaire », définie simultanément par référence à la « légitimité démocratique » et à la «subsidiarité».
} 


\section{Les risques d'une idéologie qui ne dit pas son nom}

Au regard de ces contradictions, le projet consistant à «Mieux légiférer» appelle, sinon des observations critiques, du moins certaines mises en garde. D'autant plus, d'ailleurs, qu'il est formulé en des termes consensuels qui inhibent a priori toute possibilité de désaccord : comment s'opposer à l'idée consistant à mieux légiférer? Cependant, cette évidence n'est que de façade et occulte ce qu'un tel projet peut comporter d'illusoire et de pernicieux. La démarche est illusoire si elle présume l'existence d'un consensus quant aux transformations à opérer ou l'existence d'un «mieux» objectif dont il suffirait de tirer les conséquences nécessaires. Elle est pernicieuse, aussi, en ce que le projet consiste en pratique à hisser au rang de vérités méthodologiques les valeurs de ceux qui le promeuvent $^{81}$. La récurrence des références aux besoins des entreprises - alternativement placées à égalité avec les citoyens ${ }^{82}$ et avec la société dans son ensemble ${ }^{83}$ - inscrivent nettement les théories $\mathrm{du}$ «Mieux légiférer » dans une pensée d'ordre libéral ${ }^{84}$. Cela, assurément, ne suffit pas à discréditer le projet. Cependant, cette dimension profondément idéologique du «Mieux légiférer » est voilée par le recours à une expression consensuelle qui ne peut qu'emporter l'adhésion. Cette critique a été nettement formulée par Olivier Corten à propos du processus de codification du droit international coutumier. Classiquement présenté sous le jour d'une simple «opération de conversion ${ }^{85}$ de règles coutumières en règles écrites, le processus de codification n'en comporte pas moins une importante dimension «idéologique» (idéologie par ailleurs assez proche de celle véhiculée par le Mieux légiférer ${ }^{86}$ ) qui ne saurait être niée au prétexte du prétendu caractère objectif de la méthode employée ${ }^{87}$.

Ainsi conçu, le projet consistant à «Mieux légiférer» est potentiellement porteur d'un double danger. Le premier, d'ordre pratique, est celui de l'invocation instrumentale par les États d'un prétendu «mieux objectif», qui ne serait en réalité rien d'autre que l'expression de leurs aspirations propres, sinon de leurs intérêts bien compris ${ }^{88}$. Le second danger, d'ordre épistémologique, consiste

81 Oliver CORTEN dénonce de façon analogue, à propos de la codification du droit international, un procédé «présentant comme objectives et universelles des règles qui ont été décidées par un organe composé d’à peine quinze membres » (O. CoRTEN, «Les aspects idéologiques de la codification du droit international » in Le Code Napoléon, un ancêtre vénéré? Mélanges offerts à facques VANDERLINDEN, Bruylant, Bruxelles, 2004, p. 519). Sur les hésitations et divergences quant à la nature et aux fonctions de la codification en droit international, v. également A. MAHIOU, «Rapport général» in Société française pour le droit international, La codification du droit international - Actes du colloque d'Aix-en-Provence, Pedone, Paris, 1999, p. 41 et s.

82 Le site internet du Conseil dédié au programme «Mieux légiférer » prévoit une amélioration de la réglementation opérée « en simplifiant la législation et en l'adaptant mieux aux entreprises et aux citoyens» [http://www.consilium.europa.eu/fr/policies/better-regulation; lien à jour au 5 avril 2017 (nous soulignons)].

83 Le site internet du Conseil dédié au programme «Mieux légiférer» présente comme «indispensable de veiller à la qualité de la réglementation et de faire en sorte que celle-ci réponde aux besoins de la société et des entreprises» [http://www.consilium.europa.eu/fr/ policies/better-regulation; lien à jour au 5 avril 2017 (nous soulignons)].

84 Comme le confirme par ailleurs la récurrence du lexique économique, le programme « Mieux légiférer » constituant selon les institutions de l'Union une «contribution essentielle à la compétitivité, à la croissance et à l'emploi » [http://www.consilium.europa.eu/fr/policies/betterregulation; lien à jour au 5 avril 2017]

85 P. Dailler, M. Forteau, A. Pellet, Droit international public, LGDJ, Paris, 2009, p. 336, cité in O. Corten, «Les aspects idéologiques de la codification... », précit., p. 495.

86 O. Conten, «Les aspects idéologiques de la codification...», précit., p. 520 : «Le mythe de la rationalisation et l'unification remplit plus fondamentalement un rôle qui consiste à la fois à accompagner, favoriser et légitimer une société libérale en développement, une société soucieuse de sécurité et de prévisibilité, valeurs précisément portées par la codification ». Il suffit de remplacer le dernier mot de la citation par l'expression «Mieux légiférer » pour se convaincre de la communauté de finalités des deux processus.

$87 \quad$ Ibid., p. 495.

88 V. mutatis mutandis les développements d'Olivier CoRTEN sur l'usage de la codification comme moyen pour les États de légitimer leurs «positions politiques particulières » (O. CORTEN, «Les aspects idéologiques de la codification... », précit., p. 514). 
en l'essentialisation par la doctrine d'un mode de production normative idéal, qui en viendrait à envisager les questions institutionnelles comme relevant du champ de la connaissance et non plus de la volonté, comme relevant de considérations techniques et non plus politiques ${ }^{89}$.

Il existe donc des raisons de se montrer sceptique face à ce projet consistantà « mieux » « légiférer » en droit international - en tout cas dans les termes proposés par l'Union européenne. Cela, toutefois, ne doit pas conduire à conclure à l'impossibilité - et moins encore à l'inutilité - d'une réforme en profondeur de ses modes de création du droit. Les poussées en ce sens ne manquent d'ailleurs pas : certains dénoncent aujourd'hui fermement le caractère excessivement révérencieux du droit international à l'égard du consentement des États, y compris en matière de traités ${ }^{90}$; d'autres regrettent les lenteurs du processus coutumier ${ }^{91}$. S'il est trompeur, ainsi que le soulignait Jean de Louter dès 1919, de croire que ces évolutions peuvent se faire à contresens de l'histoire de la discipline, il serait sans conteste tout aussi illusoire de penser que sa pratique, cristallisée au cours des siècles, est vouée à ne plus évoluer dans l'avenir. Les initiatives viendront sans doute, pour une part, des États et des institutions internationales elles-mêmes : le droit international sait - à sa manière, faire preuve de « créativité $»^{92}$. Cependant, les transformations des modes de création et d'application du droit international résulteront également de la capacité de la doctrine elle-même à réinventer sa manière de concevoir et de rendre compte de la pratique des acteurs internationaux ${ }^{93}$.

89 Sur cette question du rapport entre la technique et le politique dans l'organisation du pouvoir au sein de l'Union européenne, v. récemment Nilsa Rojas-Hutinel, La séparation du pouvoir dans l'Union européenne, thèse, Paris-Est Créteil, 2014, spéc. p. 19-29.

90 V. en ce sens la remarquable opinion jointe par le juge Antonio Augusto CANÇADo TrindADE au jugement de la Cour interaméricaine des droits de l'homme en l'affaire Blake c. Guatemala (objections préliminaires, 2 juillet 1996, Série C, n 27, par. 12-14). L'auteur - désormais juge à la Cour internationale de Justice - regrette l' « insufficient evolution of the precepts of the law of treaties to fulfil the basic purpose of effective protection of human rights » et invite à dépasser « the rigid postulates of the law of treaties » pour avancer « resolutely towards the gradual humanization of the law of treaties [...], as this chapter of international law persists still strongly impregnated with State voluntarism and an undue weight attributed to the forms and manifestations of consent $»$.

91 V. le classique débat, dont les termes ont été posés par René-Jean Dupuy, opposant les «coutumes sages », «lentement dégagées de faits immémoriaux », aux « coutumes sauvages » - «dont l'excroissance soudaine puise sa racine [...] dans les volontés alertées » (R.-J. DuPuY, «Coutume sage et coutume sauvage» in La communauté internationale - Mélanges offerts à Charles Rousseau, Pedone, Paris, 1974, p. 76).

92 S. Sur, La créativité du droit international, RCADI, t. 363, 2013.

93 V. pour une illustration récente É. FromagEAu, Les institutions du droit administratif global-Étude des interactions avec le droit international public, Bruylant, Bruxelles, coll. Fus gentium, 2016, 290 p. et plus spécifiquement sur les modes de création du droit dans ce contexte E. BENVENisTI, The International Law of Global Governance, RCADI, t. 368, 2013, spéc. le chapitre intitulé « Legal Regulation of the Decision-Making Process within Global Governance Bodies », p. 152 et s. 


\title{
LA CONCE PTION DU « MIEUX LÉGIFÉRER » EN DROIT CONSTITUTIONNEL
}

\author{
Marthe FAtin-Rouge Stefanini ${ }^{1}$
}

\section{Résumé}

Le droit constitutionnel est-il susceptible d'apporter une contribution efficace à l'amélioration de la qualité de la législation ? Cette question se pose au regard de la multiplication des exigences constitutionnelles destinées à améliorer le processus d'élaboration de la loi et la qualité de celle-ci, en France. Si la manière particulière dont le droit constitutionnel français s'est saisi de ces questions trouve son origine dans la volonté de rationalisation de la procédure législative et de la loi elle-même sous la $V^{e}$ République, les nombreuses exigences constitutionnelles posées ne se révèlent pas pour l'instant véritablement efficaces.

\section{Abstract}

Is constitutional law likely to make an effective contribution to improving the quality of legislation? This question arises in the light of the multiplication of constitutional requirements designed to improve the process of drafting the law and the quality of the law in France. Although the desire to rationalize the legislative procedure and the law itself under the Fifth Republic can explain the numerous constitutional requirements about lawmaking, they are not revealed truly effective.

On peut s'étonner qu'aujourd'hui, en France en particulier, la question n'est plus de savoir si le droit constitutionnel doit s'occuper de la manière de légiférer. Il semble acquis que le droit constitutionnel français s'occupe de la qualité des normes, et de la qualité des lois en particulier. Or, vingt ans en arrière, le droit constitutionnel n'aurait pas été placé au premier plan dans cette réflexion. Même si le droit constitutionnel est par nature illimité et peut s'occuper de toute question, à partir du moment où le constituant le décide, les problèmes liés à la qualité du droit n'ont été véritablement pris en considération, par le juge constitutionnel puis par le constituant lui-même, qu'à une époque récente. Donc, avant même de se demander comment le droit constitutionnel contribue à mieux légiférer et de quelle manière, la question de savoir si le droit constitutionnel est le mieux à même de

1 Directrice de recherche, Aix Marseille Univ, Université de Toulon, Univ Pau \& Pays Adour, CNRS, DICE, GERJC-ILF, Aix-en-Provence, France. 
s'occuper de la qualité des normes, ou du moins de certaines d'entre elles, se pose. Le droit constitutionnel est, dans la plupart des États, relativement rigide avec des mécanismes de révision plus ou moins lourds : de ce fait, on peut se demander s'il est opportun d'inscrire dans le texte constitutionnel, imaginé pour durer, des exigences ou des procédures visant à améliorer à la fois la manière de légiférer mais également les législations sur le plan formel et matériel. En outre, les dispositions contenues dans le texte constitutionnel sont par nature très générales et volontairement vagues : or, ces caractéristiques sont-elles bien compatibles avec les nécessités de la légistique ? Enfin, le contrôle de constitutionnalité et les sanctions qui l'accompagnent, que ce soit l'annulation, l'abrogation ou l'interprétation conforme de la disposition mal écrite ou mal conçue, sont-ils bien adaptés?

En somme, le droit constitutionnel est-il susceptible d'apporter une contribution efficace à l'amélioration de la qualité de la législation?

La démarche de droit comparé, méthode très éclairante et certainement attendue, n'est pas celle que l'on peut adopter tant les spécificités du droit français sur cette question sont marquées. Non point que la question de l'appréhension constitutionnelle de la qualité des normes, et des lois en particulier, soit spécifiquement française mais parce qu'aucun autre État, à notre connaissance, ne s'est saisi au niveau constitutionnel, de la question de la qualité de la législation de la même façon qu'en France. En effet, si les questions de légistique d'un point de vue technique ont fait l'objet d'attention dans plusieurs États ${ }^{2}$, les dispositions qui constituent dans la plupart des cas du droit souple, des recommandations, des codes de bonne conduite, n'ont pratiquement jamais d'assise constitutionnelle. On trouve bien le principe d'une évaluation de l'efficacité de la législation à l'article 170 de la Constitution suisse ${ }^{3}$ mais ce type de disposition est encore rare dans les Constitutions. En revanche, les exigences de clarté, d'intelligibilité et de précision des lois, qui visent la qualité rédactionnelle des lois, se retrouvent dans plusieurs systèmes juridiques garantis par la juridiction constitutionnelle (principe de clarté, de précision et de déterminabilité de la règle de droit en Allemagne, principe de clarté, de précision et de prévisibilité de la loi pénale en Belgique, principe de clarté, d'intelligibilité et de prévisibilité de la loi pénale en Italie ${ }^{4}$, nullité de l'imprécision au Canada ${ }^{5}$, principe de clarté et de non-confusion normative en Espagne ${ }^{6}$, vagueness doctrine aux États-Unis ${ }^{7}$, par exemple). Ces exigences sont particulièrement présentes en matière pénale et le sont plus généralement en matière de droits fondamentaux.

2 C'est le cas, par exemple, de l'Allemagne, de l'Autriche, de la Belgique, du Canada, des États-Unis, de l'Espagne, de l'Italie, du Portugal et de la Suisse : Voir notamment, J.-L. Bergel, "Esquisse de méthodologie législative comparée », in M. Fatin-Rouge Stefanini, L. Gay, J. Pini, (éd.), Autour de la qualité des normes, Bruylant, Bruxelles, 2010, p. 145-161 ; M. FATIN-Rouge StefaniNi, «Variabilité et contingence des exigences de qualité. Quelques considérations sur la notion de qualité des normes en droit constitutionnel », in Autour de la qualité des normes, précité, p. 32-36 ; L. GAY, «Le contrôle des qualités formelles de la loi en droit constitutionnel comparé : France, Espagne et Canada », in Autour de la qualité des normes, précité, p. 97-144; A. FLUECKIGER, "Qu'est que mieux légiférer veut dire ? Enjeux et instrumentalisation de la notion de qualité législative », in FluecKIGER et al. (éd.), Guider les parlements et les gouvernements pour mieux légiférer : le rôle des guides de légistique, Genève, Schulthess, 2008, p. 11-32 ; A. FLUECKIGER et S. GrodecKI, « La clarté : un nouveau principe constitutionnel ? », Revue de Droit Suisse, 2017, vol. 136, p. 31-62 ; P. DE Montalivet, « La « juridicisation » de la légistique. À propos de l'objectif de valeur constitutionnelle d'accessibilité et d'intelligibilité de la loi », in R. DrAGo (éd.), La confection de la loi, PUF, Cahiers des sciences morales et politiques, 2005 , p. 99-136.

3 L'article 170 de la Constitution dispose : «L'Assemblée fédérale veille à ce que l'efficacité des mesures prises par la Confédération fasse l'objet d'une évaluation ».

M. Fatin-Rouge Stefanini, précité, p. 35.

L. GAY, précité.

L. GAY, précité.

P. RrAPI, L'accessibilité et l'intelligibilité de la loi en droit constitutionnel. Étude du discours sur la « qualité de la loi », LGDJ, 2014, p. 141-168. 
La manière particulière dont le droit constitutionnel français s'est saisi des questions de qualité de la production législative trouve son origine dans la volonté de rationalisation de la procédure législative et de la loi elle-même ; rationalisation qui caractérise la Ve République par rapport aux Républiques précédentes.

Après avoir rappelé les causes, bien connues, de cette rationalisation (1), en seront expliquées les conséquences c'est-à-dire le développement d'un certain nombre de normes constitutionnelles destinées à améliorer la qualité de la loi (2). Enfin, force est de constater que si les exigences constitutionnelles destinées à contribuer à la qualité de la loi sont nombreuses, elles ne sont pas pour l'instant véritablement efficaces (3).

\section{1) Les causes du particularisme français : des dispositions constitutionnelles rationnalisant le travail du Parlement}

La rationalisation du travail du Parlement sous la Ve République trouve sa source dans la volonté de réagir aux excès des Républiques précédentes et s'est traduite de trois façons sur le plan constitutionnel : une limitation du domaine de la loi ; l'organisation et l'encadrement de la procédure législative ; la mise en place d'un contrôle $a$ priori de la constitutionnalité des lois.

Tout d'abord, la limitation du domaine de la loi est une caractéristique de la Constitution de 1958. Dans l'esprit et la lettre même de la Constitution de la Ve République, le domaine de la loi a été limité de façon verticale et horizontale : verticalement, en imposant au législateur le respect de la suprématie de la Constitution dans la hiérarchie des normes; horizontalement, en définissant le domaine de la loi à l'article 34 de la Constitution et en introduisant les règlements dits autonomes de l'article 37 al 1 qui dispose que « Les matières autres que celles qui sont du domaine de la loi ont un caractère réglementaire ». Il en a été déduit que les matières qui ne sont pas du domaine de la loi sont du domaine du règlement. Cette limitation ne pouvait être effective sans un organe chargé de contrôler le bon respect des règles établies, ce qui a justifié la mise en place du Conseil constitutionnel dont la mission principale était, à l'origine, la « régulat(ion) de l'activité des pouvoirs publics ${ }^{8}$. Cela a eu des conséquences importantes : en limitant le domaine de la loi, le constituant a posé une première règle décisive à l'intention des membres du Parlement des futures législatures de la $V^{e}$ République : le Parlement ne peut pas légiférer sur tout.

À l'inverse, certaines questions relèvent de la compétence exclusive du législateur et donc ne peuvent être laissées à l'appréciation d'autres autorités. Cela donnera lieu à la jurisprudence relative aux incompétences négatives consistant pour le Conseil constitutionnel à censurer une disposition législative dans laquelle le législateur n'épuiserait pas la compétence qu'il tient de la Constitution?.

\footnotetext{
$8 \quad$ Formule employée par le Conseil constitutionnel lui-même dans la décision n 62-20 DC du 6 novembre 1962, Loi relative à l'élection du Président de la République au suffrage universel direct, cons. 2. V. notamment, L. FAvoreu, « Le Conseil constitutionnel régulateur de l'activité normative des pouvoirs publics », Revue du droit public et de la science politique en France et à l'étranger, 1967, p. 5-120.

9 Voir ci-dessous.
} 
La volonté, du constituant, de rationaliser la procédure législative s'est traduite à la fois par des dispositions constitutionnelles qui n'ont cessé de se renforcer mais également par la soumission des règlements d'assemblée, et de toutes leurs modifications, au contrôle de constitutionnalité ${ }^{10}$. Cette compétence confiée à la juridiction constitutionnelle semble être une spécificité française ${ }^{11}$. Les autres juridictions constitutionnelles d'une manière générale ne sont pas compétentes pour connaître des règlements d'assemblée car la tendance est de considérer que ces règlements font partie en quelque sorte de la « cuisine interne » des assemblées. Le contrôle des règlements d'assemblée procède directement du souci de rationaliser la procédure législative en 1958. Or, ce contrôle a conduit le Conseil constitutionnel à façonner progressivement la procédure législative et a eu un impact direct sur la confection des lois.

Enfin, la rationalisation du travail du Parlement a été renforcée par un troisième facteur : le contrôle a priori de constitutionnalité des lois ${ }^{12}$, c'est-à-dire un contrôle de constitutionnalité exercé avant l'entrée en vigueur d'une loi, (ce qui constituait la seule manière de contrôler la constitutionnalité des lois avant l'introduction de la QPC en 2010). Dans le cadre de cette compétence, le Conseil constitutionnel a dirigé son contrôle non seulement sur le fond des lois mais également sur la procédure et sur la forme. Par conséquent, une loi peut ne pas entrer en vigueur parce qu'un certain nombre d'exigences procédurales dans la manière dont le processus législatif s'est déroulé, dans la manière dont la loi a été confectionnée, n’ont pas été respectées.

Or, nulle part ailleurs la juridiction constitutionnelle ne s'est autant immiscée dans la procédure législative et cela a eu des conséquences importantes dont la rationalisation de la production législative elle-même ${ }^{13}$.

\section{2) Les conséquences du particularisme français : le développement de normes constitutionnelles destinées à régir la production législative}

Il existe plusieurs normes constitutionnelles qui concourent à la qualité de la loi en France. Ces normes se trouvent soit directement inscrites dans la Constitution, soit dans la jurisprudence du Conseil constitutionnel. À ce propos, la question de la qualité des lois en France a été beaucoup discutée sur le plan constitutionnel à partir de la fin des années 1990 et du début des années 2000. Cela tient surtout à la volonté du Président du Conseil constitutionnel de l'époque, Pierre Mazeaud, d'utiliser tous les moyens que la juridiction avait à sa disposition pour lutter contre un phénomène à l'encontre duquel il ne dissimulait pas un profond agacement ${ }^{14}$. Cela est aussi très révélateur de la place centrale qu'a longtemps occupée la loi en France, et qui avait conduit à parler de légicentrisme sous les Républiques précédentes. Or, prêter une attention particulière aux qualités rédactionnelles

\footnotetext{
10 Voir en particulier S. HUTIER, Le contrôle de la procédure parlementaire par le Conseil constitutionnel, LGDJ, Institut universitaire Varenne, 2016, 648 p.

11 S. De Cacoueray, Le Conseil constitutionnel et les règlements des Assemblées, Economica, collection Droit public positif, 2001, 472 p. Article 61 al. 1 et 2 de la Constitution.

Voir S. HuTiER, précité.

Vœux du Président du Conseil constitutionnel, Pierre Mazeaud, au Président de la République, 3 janvier 2005, Les Cahiers du Conseil constitutionnel, $\mathrm{n}^{\circ} 18,2005$, p. 3-18.
} 
de la loi est un moyen de lutter contre le déclin de la loi. En effet, la dégradation de la loi participe à sa démystification au profit d'autres types de normes, et contribue à affaiblir la confiance qu'ont les citoyens envers les gouvernants et les représentants.

En réalité trois phases peuvent être distinguées et montrent une construction par étage des exigences destinées à favoriser une amélioration la qualité des normes.

\subsection{La première période est celle allant de l'adoption de la Constitution de 1958 à 1998 et, précisément, à la décision du Conseil constitutionnel $n^{\circ}$ 98-401 DC du 10 juin 1998}

Dans cette première période, le Conseil constitutionnel veille à la qualité de la loi sans prononcer ces termes.

En effet, aucune qualité formelle de la loi ne se trouve affirmée dans la Constitution de 1958 ; en revanche, des qualités matérielles découlant du texte constitutionnel peuvent être relevées pour la loi en général, et pour la loi pénale en particulier.

L'irrecevabilité législative de l'article 41 de la Constitution, par exemple, va permettre d'écarter des dispositions, et notamment des amendements, qui ne relèveraient pas du domaine de la $\operatorname{loi}^{15}$. Dans la plupart des cas, l'irrecevabilité est traitée en interne par les assemblées, sans que le Conseil constitutionnel ne soit saisi ${ }^{16}$. La procédure de délégalisation de l'article 37 al. 2 a le même objet, préserver le domaine du règlement en permettant au Conseil constitutionnel d'examiner si les dispositions qui lui sont soumises relèvent du domaine de la loi ou du domaine du règlement, mais à la différence de la procédure de l'article 41, ce contrôle intervient une fois la loi adoptée.

Cette attention portée à la qualité matérielle de la loi, découlant des compétences que le Conseil constitutionnel tient du constituant, trouve cependant ses limites dans le contrôle de constitutionnalité. En effet, par une décision de 1982, Blocage des prix et des revenus ${ }^{17}$, le Conseil constitutionnel a considéré qu'une loi contenant des dispositions du domaine règlementaire n'est pas pour autant inconstitutionnelle ; il refuse donc de sanctionner l'empiètement du législateur sur le domaine du règlement. Il infléchira en partie cette jurisprudence en 2005 dans le but précisément d'améliorer la qualité de la loi ${ }^{18}$.

Le Conseil constitutionnel est allé plus loin et a lui-même développé une jurisprudence contribuant à favoriser la qualité matérielle de la législation. Ceci est le cas du grief d'incompétence négative qui peut être soulevé dans le cadre du contrôle de constitutionnalité des lois ordinaires ou des lois

15 L'article 41 de la Constitution dispose : « S'il apparaît au cours de la procédure législative qu'une proposition ou un amendement n'est pas du domaine de la loi ou est contraire à une délégation accordée en vertu de l'article 38, le Gouvernement ou le président de l'assemblée saisie peut opposer l'irrecevabilité. En cas de désaccord entre le Gouvernement et le président de l'assemblée intéressée, le Conseil constitutionnel, à la demande de l'un ou de l'autre, statue dans un délai de huit jours ».

16 Le Conseil constitutionnel a été saisi pour la dernière fois en 1979 sur le fondement de cette disposition.

17 Décision $\mathrm{n}^{\circ}$ 82-143 DC du 30 juillet 1982, Blocage des prix et des revenus, cons. 11.

18 Décision n ${ }^{\circ}$ 2005-512 DC du 21 avril 2005, Avenir de l'École, cons. 23 et article 3 du dispositif. Voir J.-M. AuBY, « L'avenir de la jurisprudence Blocage des prix et des revenus », Cahiers du Conseil constitutionnel, ${ }^{\circ}$ 19, 2006 (en ligne sur le site du Conseil constitutionnel). 
organiques. En effet, le Conseil constitutionnel accepte de censurer, depuis $1967^{19}$, le fait, pour le législateur, de laisser à l'appréciation d'une autre autorité une règle ou un principe pour laquelle il est seul et exclusivement compétent pour décider. Cela suppose que le législateur détermine de façon suffisamment précise les conditions de mise en œuvre des règles ou principes qu'il pose. Ainsi, si la règle posée par le législateur est floue, susceptible de plusieurs interprétations, le Conseil constitutionnel peut censurer cette disposition pour incompétence négative. Il en résulte que le législateur doit être précis lorsqu'il est exclusivement compétent ${ }^{20}$ et doit l'être d'autant plus que des droits et libertés fondamentaux sont en jeu. Le grief d'incompétence négative est souvent soulevé, il peut l'être désormais dans le cadre d'une QPC dans la mesure où est invoquée une atteinte à un droit ou une liberté constitutionnellement garantie, et a donné lieu à de nombreuses censures. Ce grief s'avère être l'un des principaux outils dont dispose le Conseil constitutionnel pour contribuer à l'amélioration de la qualité de la loi.

Le Conseil constitutionnel a également développé une jurisprudence sur le droit d'amendement, qui permet d'éviter que certains amendements alourdissent la procédure législative, d'une part, et le texte lui-même, d'autre part. Les exigences posées en la matière n'ont cessé de se renforcer à partir des années $1980^{21}$ face à la technique de l'obstruction législative, au développement des cavaliers, c'est-à-dire de l'ajout par voie d'amendement de dispositions sans rapport avec le texte en discussion ${ }^{22}$ et, plus généralement, face à l'ensemble des manœuvres provenant de la majorité ou de l'opposition et ayant pour effet d'alourdir la procédure et le texte lui-même.

En ce qui concerne la loi pénale, l'exigence de clarté et de précision de la loi est apparue dans la jurisprudence du Conseil constitutionnel à partir des années 1980. Cette exigence a été directement reliée au principe de nécessité des peines proclamé par l'article 8 de la Déclaration des droits de l'Homme et du citoyen. Dans la décision Sécurité et liberté du 20 janvier $1981^{23}$ notamment, le Conseil constitutionnel souligne qu' " aux termes de l'article 8 de la Déclaration des droits de l'Homme et du citoyen de 1789 nul ne peut être puni qu'en vertu d'une loi établie et promulguée antérieurement au délit et légalement appliquée ; qu'il en résulte la nécessité pour le législateur de définir les infractions en termes suffisamment clairs et précis pour exclure l'arbitraire ». L'exigence de clarté et de précision de la loi pénale est une expression de la protection du domaine réservé à loi ${ }^{24}$, souci que l'on retrouvera plus largement exprimé dans la deuxième période.

19 J. Trémeau, La réserve de loi. Compétence législative et Constitution, Economica, PUAM, Aix-en-Provence, 1997,414 p.

20 La première censure est intervenue dans la décision $\mathrm{n}^{\circ} 75-56 \mathrm{DC}$ du 23 juillet 1975, Loi modifiant et complétant certaines dispositions de procédure pénale.

21 S. Hutier, précité, p. 305. Voir également M. Proкоріак, L’amélioration de la qualité rédactionnelle des textes législatifs, Approche comparée Droit français - Droit de l'Union européenne, thèse dactyl. Limoges, 2015, p. 282.

22 Décision $\mathrm{n}^{\circ}$ 86-221 DC du 29 décembre 1986, Loi de finances pour 1987, cons. 5. Jurisprudence sur les limites inhérentes au droit d'amendement qui sera abandonnée à partir de la décision 1998-402 DC du 25 juin 1998, Loi portant diverses dispositions d'ordre économique et financier, cons. 3.

23 Décision n ${ }^{\circ}$ 80-127 DC du 20 janvier 1981, Sécurité et liberté, cons. 7.

24 Voir P. RRAPI, précité, p. 47 ; J. TRÉMEAU, précité, p. 267. 


\subsection{La deuxième période court de la décision du 10 juin 1998 jusqu’à la révision constitutionnelle du 23 juillet 2008}

Cette période se caractérise par une forme d'insurrection du Conseil constitutionnel, contre la dégradation de la qualité de la loi, sous l'impulsion de son Président qui fait clairement état de sa colère vis-à-vis de cette situation ${ }^{25}$. Devenues objet de communication politique, les lois sont de plus en plus nombreuses ; répondant à un événement plus qu'à une nécessité, certaines deviennent rapidement obsolètes ou sont sans intérêt. Les modifications fréquentes rendent le droit imprévisible et perturbent la sécurité juridique. En outre, la pratique des cavaliers législatifs, sociaux et budgétaires n'a cessé de se développer. Le Conseil constitutionnel va alors dégager un certain nombre de principes, objectifs et exigences constitutionnelles destinés à exercer une forme de pression sur les différents acteurs du processus législatif.

- Le premier principe posé est celui de la clarté de la loi par la décision n 98-401 DC du 10 juin 1998 portant sur la Loi d'orientation et d'incitation à la réduction du temps de travail26. Le Conseil constitutionnel accepte un grief tiré de la méconnaissance de l'exigence constitutionnelle de clarté de la loi, en dehors de la matière pénale, en se fondant sur la compétence que le législateur tire de l'article 34 de la Constitution et qui détermine le domaine de la loi. Une première censure est intervenue sur ce fondement en $2000^{27}$. Toutefois, ce principe sera critiqué par une partie des membres de la doctrine universitaire en raison de son caractère vague et de la redondance qu'il entretient avec un objectif consacré une année plus tard ${ }^{28}$.

- En effet, en 1999, le Conseil constitutionnel, à l'occasion de la Loi portant habilitation du Gouvernement à procéder, par ordonnances, à l'adoption de la partie législative de certains codes, énonce un objectif de valeur constitutionnelle d'accessibilité et d'intelligibilité de la loi qu'il fonde sur quatre dispositions : les articles 4, 5, 6 et 16 de la Déclaration de 178929. Le Conseil constitutionnel estime «qu'en effet l'égalité devant la loi énoncée par l'article 6 de la Déclaration des droits de l'homme et du citoyen et « la garantie des droits « requise par son article 16 pourraient ne pas être effectives si les citoyens ne disposaient pas d'une connaissance suffisante des normes qui leur sont applicables ; qu'une telle connaissance est en outre nécessaire à l'exercice des droits et libertés garantis tant par l'article 4 de la Déclaration, en vertu duquel cet exercice n'a de bornes que celles déterminées par la loi, que par son article 5, aux termes duquel «tout ce qui n'est pas défendu par la loi ne peut être empêché, et nul ne peut être contraint à faire ce qu'elle n’ordonne pas « ». Selon Patricia Rrapi, en choisissant cet objectif au détriment du principe de clarté, le Conseil constitutionnel opère un choix

\footnotetext{
25 Pierre Mazeaud dans les voeux adressés au Président de la République, le 3 janvier 2005, n'hésite pas à parler de « colère sacrée » vis-à-vis des «neutrons législatifs » et rappelle que la loi doit «non seulement être précise mais également revêtue d'une portée normative ». 26 Cons. $\mathrm{n}^{\circ} 10$ : «Considérant, en premier lieu, que l'article 1er critiqué fixe au 1er janvier 2000 ou au 1er janvier 2002, selon l'effectif des entreprises en cause, l'entrée en vigueur de la réduction de la durée légale de travail effectif des salariés de trente-neuf heures à trente-cinq heures par semaine ; qu'il était loisible au législateur, sans méconnaître aucun principe, ni aucune règle constitutionnelle, de donner à cette mesure, qui, en l'état, est définie de façon suffisamment claire et précise pour satisfaire aux exigences découlant de l'article 34 de la Constitution, un effet différé ».

27 Décision n²000-435 DC du 7 décembre 2000, Loi d'orientation pour l'Outre-Mer, cons. 53.

28 Voir notamment A. Flueckiger, «Le principe de clarté de la loi ou l'ambiguïté d'un idéal », Cahiers du Conseil constitutionnel, $\mathrm{n}^{\circ} 21$, 2007, en ligne sur le site du Conseil constitutionnel ; L. Milano, «Contrôle de constitutionnalité et qualité de la loi », Revue de droit public et de sciences politiques, 2006, p. 643-646; P. DE MONTALIVET, précité, p. 127.

29 Décision n ${ }^{\circ}$ 99-421 DC du 16 décembre 1999, Codification par ordonnances, cons. 13.
} 
de politique jurisprudentielle apparaissant « comme correspondant davantage à l'air du temps » et faisant écho à l'« idée d'amélioration préjuridique des rapports du citoyen au droit » ${ }^{30}$.

Le but de cet objectif de valeur constitutionnelle a été lui-même explicité au fil de la jurisprudence. Le Conseil constitutionnel affirme qu'il s'agit de «prémunir les sujets de droit contre une interprétation contraire à la Constitution ou contre le risque d'arbitraire, sans reporter sur les autorités administratives ou juridictionnelles le soin de fixer des règles dont la détermination n'a été confiée par la Constitution qu'à la loi $»^{31}$. Le Conseil constitutionnel vérifie notamment que les dispositions sont « précises » et «non équivoques », n'ont pas de caractère « ambigu » ou « obscur » ${ }^{32}$, ne contiennent pas de contradictions ${ }^{33}$ ou encore ne sont pas excessivement complexes ${ }^{34}$. Cet objectif a conduit à un certain nombre de censures dans le cadre du contrôle de constitutionnalité $a$ priori ${ }^{35}$.

L'objectif de valeur constitutionnelle d'accessibilité et d'intelligibilité de la loi peut également être invoqué dans le cadre du contrôle a posteriori ( $\mathrm{QPC})$, dans la mesure où un droit ou une liberté s'en trouverait affecté ${ }^{36}$.

- À partir de 2004, le Conseil constitutionnel pose l'exigence de normativité de la loi, qu'il accepte de censurer dans le cadre de son contrôle de constitutionnalité $a$ priori ${ }^{37}$. Cette exigence est destinée à lutter contre les «neutrons législatifs », d'après l'expression de Jean Foyer, c'est-à-dire des dispositions trop vagues, au contenu indéterminé qui posent des difficultés d'interprétation pour ceux qui ont à les appliquer. Alors qu'auparavant le Conseil constitutionnel pratiquait la technique de la déclaration d'inopérance ou celle de la réserve neutralisante vis-à-vis de telles dispositions, le passage à la censure marque un durcissement de la politique jurisprudentielle de ce dernier vis-à-vis des pratiques législatives. Cette exigence est rattachée à l'article 6 de la Déclaration des droits de l'homme et du citoyen de 1789. Selon le Conseil constitutionnel « [...] aux termes de l'article 6 de la Déclaration des droits de l'homme et du citoyen de 1789: « La loi est l'expression de la volonté générale »; qu'il résulte de cet article comme de l'ensemble des autres normes de valeur constitutionnelle relatives à l'objet de la loi que, sous réserve de dispositions particulières prévues par la Constitution, la loi a pour vocation d'énoncer des règles et doit par suite être revêtue d'une portée normative ». Le contenu de cette exigence étant lui-même difficile à déterminer, le Conseil

$30 \quad$ Précité, p. 53

31 V. notamment la décision 2006-540 DC du 27 juillet 2006, Droits d'auteur, cons. 9.

32 V. notamment les décisions 2011-629 DC du 12 mai 2011, Loi de simplification et d'amélioration de la qualité du droit, cons. 21 ; 2012-659

DC du 13 décembre 2012, Loi de financement de la sécurité sociale pour 2013, cons. 60. Voir plus largement P. RRAPI, précité.

33 Décision $n^{\circ}$ 2012-662 DC du 29 décembre 2012, Loi de finances pour 2013, cons. 84

34 Décision n ${ }^{\circ}$ 2005-530 DC du 29 décembre 2005, Loi de finances pour 2006, cons. 77 et 78. La simple complexité du texte ne suffit pas à elleseule à porter atteinte à l'OVC (Décision 2011-629 DC, précité, cons. 6 ; 2012-662DC du 29 décembre 2013, Loi de finances pour 2013, cons. 119). 35 V. notamment pour les plus récentes : 2011-639 DC du 28 juillet 2011, Politique du handicap, cons. 10; 2011-644 DC du 28 décembre 2011, Loi de finances pour 2012, cons. 16 ; 2012-662 DC du 29 décembre 2012, Loi de finances pour 2013, cons. 52 et 84 ; $2013-675$ DC du 9 octobre 2013, Loi organique relative à la transparence de la vie publique, cons. 67 ; 2013-685 DC du 29 décembre 2013, Loi de finances pour 2014, cons. 130 ; 2014-708 DC du 29 décembre 2014, Loi de finances rectificative pour 2014, cons. 41 ; 2016-741 DC du 8 décembre 2016, Loi relative à la transparence, à la lutte contre la corruption et à la modernisation de la vie économique, cons. 146 ; 2016-745 DC du 26 janvier 2017, Loi relative à l'égalité et à la citoyenneté, cons. 121. Pour une réserve d'interprétation sur ce fondement, voir 2016-739 DC du 17 novembre 2016, Loi de modernisation de la justice du XXI siècle, cons. 99.

36 V. 2012-285 QPC du 30 novembre 2012, cons. 12.

37 Décision n ${ }^{\circ}$ 2004-500 DC, 29 juill. 2004, Loi organique relative à l'autonomie financière des collectivités locales, cons. 12. Voir V. CHAMPEILDesplats, « N'est pas normatif qui peut. L'exigence de normativité dans la jurisprudence du Conseil constitutionnel », Les Cahiers du Conseil constitutionnel, $\mathrm{n}^{\circ} 21,2007$ (disponible en ligne sur le site du Conseil constitutionnel). 
constitutionnel dispose d'une marge d'appréciation importante dont il n'a pas souhaité abuser, tout comme pour l'objectif de valeur constitutionnelle d'accessibilité et d'intelligibilité de la loi. Ainsi, quatre censures sont intervenues sur ce fondement entre 2004 et $2017^{38}$.

- À partir de 2005, le Conseil constitutionnel va également imposer l'exigence de clarté et de sincérité du débat parlementaire ${ }^{39}$ qui participe à une amélioration de la production législative par une vigilance accordée par le Conseil constitutionnel au respect des règles de procédures. Cette exigence, interprétée tout d'abord dans le cadre de l'utilisation du droit d'amendement en cours de procédure législative, sera progressivement étendue à l'ensemble des procédures parlementaires dont les travaux en commission ${ }^{40}$. Elle est fondée sur l'article 6 de la DDHC de 1789 aux termes duquel : « La loi est l'expression de la volonté générale... » et l'alinéa premier alinéa de l'article 3 de la Constitution qui dispose que «La souveraineté nationale appartient au peuple qui l'exerce par ses représentants... ». Le contrôle effectué par le Conseil constitutionnel sur le fondement de cette exigence est restreint.

- Le Conseil constitutionnel va également clarifier et stabiliser sa jurisprudence relative aux limites du droit d'amendement en posant plusieurs principes ${ }^{41}:$ l'exigence d'un lien dès la première lecture entre l'amendement et le texte restant en discussion ${ }^{42}$, d'une part, et, d'autre part, la règle de l'entonnoir en précisant que « les adjonctions ou modifications qui peuvent être apportées après la première lecture par les membres du Parlement et par le Gouvernement doivent être en relation directe avec une disposition restant en discussion », mis à part pour « les amendements destinés à assurer le respect de la Constitution, à opérer une coordination avec des textes en cours d'examen ou à corriger une erreur matérielle $»^{43}$. Le contrôle particulièrement rigoureux qui sera pratiqué par le juge, n'hésitant pas à vérifier d'office le respect du cadre posé, s'inscrit lui aussi dans la volonté d'améliorer la qualité rédactionnelle des textes législatifs ${ }^{44}$.

\subsection{La troisième période s'ouvre avec la révision constitutionnelle du 23 juillet $2008^{45}$}

Le Comité Balladur qui avait travaillé sur les propositions de réforme visant à moderniser et à rééquilibrer les institutions de la Ve République a, tout en réfléchissant à la manière de revaloriser le Parlement, pris en compte la nécessité de contribuer à une amélioration de la qualité de la législation ${ }^{46}$. Cela s'est traduit par plusieurs mécanismes visés soit directement par la Constitution, soit mis en

\footnotetext{
38 Décision n 2005-512 DC du 21 avril 2005, Avenir de l'École cons. 17 ; décision n 2012-647 DC du 28 février 2012, Loi visant à réprimer la contestation de l'existence de génocide reconnus par la loi, cons. 6 ; décision n 2016-741 DC du 8 décembre 2016, précité, cons. 99 et décision 2016-745 DC du 26 janvier 2017, précité, cons. 167.

39 Cette exigence sera d'abord limitée à la sincérité des débats (décision 2005-512 DC, précité, cons. 4) puis sera intitulée « clarté et sincérité du débat parlementaire » dès la décision n $\mathrm{n}^{\circ} 2005-526$ DC du 13 octobre 2005, Résolution modifiant le règlement de l'Assemblée nationale.

40 S. Hutier, précité, p. 334-343.

41 Pour plus de détail, v. S. Hutier, précité, p. 309 et s.

42 Décision $\mathrm{n}^{\circ}$ 2005-532 DC du 19 janvier 2006, Loi relative à la lutte contre le terrorisme, cons. 6

43 Idem, cons. 26.

44 S. Hutier, précité, p. 312.

45 Loi constitutionnelle ${ }^{\circ}$ 2008-724 de modernisation des institutions de la Ve République.

46 E. BAlladur, Une Ve République plus démocratique-Comité de réflexion et de proposition sur la modernisation et le rééquilibrage des institutions de la $V^{e}$ République (notamment chapitre II, B- L'amélioration du travail législatif), disponible sur http://www.ladocumentationfrancaise.fr/ rapports-publics/074000697/index.shtml (consulté le 15 mars 2017).
} 
place par la loi organique du 15 avril 2009 relative à l'application des articles 34-1, 39 et 44 de la Constitution ${ }^{47}$ et précisés dans les règlements des assemblées.

On y trouve :

- l'introduction d'un droit de résolution ouvert aux parlementaires qui tout en redonnant la parole à ces derniers devait permettre de décharger les lois de dispositions non normatives ou sans rapport avec le texte en discussion (art. 34-1 de la Constitution);

- l'instauration, par renvoi à la loi organique, de règles de présentation des projets de loi (art. 39 al. 3 de la Constitution) qui donneront lieu notamment à l'obligation de réaliser des études d'impact. Le non-respect de ces règles peut conduire à la non-inscription des projets de loi à l'ordre du jour et, si nécessaire, à la saisine du Conseil constitutionnel (art. 39 al. 4 de la Constitution) ;

- la possibilité de soumettre les propositions de lois pour avis préalable au Conseil d'État (article 39 al. 5 de la Constitution), afin de bénéficier de l'expertise technique de ce dernier. Cela n'existait jusqu'alors que pour les projets de lois qui doivent être soumis de manière obligatoire au Conseil d'État ;

- la revalorisation du travail en commission : la discussion portant sur le texte tel qu'adopté par la commission compétente (art. 42-1 de la Constitution);

- un délai de réflexion imposé avant la discussion d'un texte, afin de permettre de décider plus sereinement de son opportunité et de sa pertinence (art. 42 al. 3 de la Constitution).

Plus particulièrement la loi organique du 15 avril 2009 va mettre en place :

- l'obligation de faire précéder le projet de loi d'un exposé des motifs (article 7 de la Loi organique) afin de favoriser l'élimination des dispositions non normatives ;

- les études d'impact introduites par l'article 8 : à la différence des études qui existaient auparavant, elles deviennent systématiques et obligatoires, mises à part les dérogations prévues par la loi organique elle-même. Elles visent à exposer non seulement l'intérêt et la nécessité de la loi mais également les choix opérés et, plus largement, les conséquences de l'insertion d'un nouveau texte dans l'ordre juridique existant. Cependant, les amendements, les propositions de loi et les ordonnances de l'article 38 de la Constitution y échappent ;

- l'encadrement du temps de la discussion législative et, en particulier, la faculté laissée aux assemblées de programmer la durée des débats (procédure du temps législatif programmé). Le but était, par une réduction du temps des débats, de lutter contre l'obstructionnisme et d'obliger les parlementaires à se concentrer sur l'essentiel, le contenu du dispositif proposé par le texte en discussion.

Dans cette révision, le constituant opère surtout un renvoi à la loi organique qui précise les conséquences de dispositions constitutionnelles très évasives destinées à la fois à revaloriser le Parlement mais également à améliorer la qualité de la production législative. Or, ces objectifs peuvent s'avérer

$47 \quad$ Loi organique $n^{\circ} 2009-403$ du 15 avril 2009. 
parfois contradictoires car multiplier les règles destinées à favoriser une production législative de meilleure qualité risque de se faire au détriment de l'autonomie et de la liberté des assemblées. Ces règles ont été fixées par le législateur organique, plus à même de prévoir des dispositions de détail, et les assemblées elles-mêmes dans le cadre de leur règlement d'intérieur. Ceci est très révélateur des limites du droit constitutionnel lui-même dans sa capacité de gérer les problèmes liés à une dégradation de la qualité de la loi.

\section{3) La faible efficacité du dispositif mis en place}

Au terme de quelques années de fonctionnement de ces dispositifs émanant de normes constitutionnelles, force est de constater que leur efficacité est relativement faible pour plusieurs raisons.

Tout d'abord, les dispositions constitutionnelles ne peuvent pas tout régir. En effet, la vocation de la norme constitutionnelle est d'être générale et abstraite, qualité sans laquelle cette norme est susceptible d'être fréquemment révisée. Par conséquent, une fois les règles posées, ce sont les autorités d'application qui sont chargées d'en assurer la concrétisation et l'efficacité. Cela est d'autant plus vrai dans une matière où les assemblées doivent disposer d'une certaine marge de manœuvre sans quoi l'effort de revalorisation du Parlement serait vain et surtout l'autonomie du Parlement serait compromise.

Cependant, l'écueil de cette marge de manœuvre, nécessaire, laissée aux assemblées est que les règles posées sont parfois contournées ou détournées en pratique donc peu efficaces. Ceci est le cas du temps législatif programmé, qui n'a pas permis, par exemple, la diminution de l'usage du droit d'amendement. Ceci est également le cas des études d'impact qui, si elles constituent une avancée majeure par leur introduction dans une loi organique à laquelle la Constitution renvoie, devraient être sensiblement améliorées. Elles devraient en particulier être réalisées par une autorité indépendante, et non par le gouvernement, et dans une approche critique et non de justification du projet de $\operatorname{loi}^{48}$. Elles devraient également pouvoir concerner les amendements et intervenir à divers stades de la procédure au fur et à mesure que le texte évolue. En outre, malgré la compétence dont il dispose, le Conseil constitutionnel assure un contrôle minimal de l'obligation d'étude d'impact ${ }^{49}$ en jugeant notamment que « le caractère éventuellement incomplet de l'étude d'impact dans l'état antérieur à son dépôt sur le bureau de la première assemblée saisie est sans incidence sur le respect des exigences de l'article 8 de la loi organique du 15 avril $2009 »^{50}$. Il se borne à vérifier l'existence même de l'étude d'impact. Force est de constater qu'il ne dispose en outre ni des moyens, ni de la légitimité nécessaires à un contrôle véritable du contenu de l'étude d'impact et ne s'aventure donc pas sur le

48 Voir notamment B.-L. Combrade, L'obligation d'étude d'impact des projets de loi, LGDf, Paris, collection Nouvelle Bibliothèque des thèses, vol. 163, 2017, 500 p. et du même auteur, « «L'étude d'impact au Parlement français : un instrument de mutation du rôle des assemblées dans le processus législatif? », Revue française d'administration publique, 2014/1, n 149, p. 195-206.

49 Voir sur ce point les décisions : 2014-12 FNR du $1^{\text {er }}$ juillet 2014, Présentation du projet de loi relatifà la délimitation des régions, aux élections régionales et départementales et modifiant le calendrier électoral (le Conseil constitutionnel a été saisi sur le fondement de l'article 39 al. 4 de la Constitution) ; 2013-683 DC du 16 janvier 2014, Loi concernant l'avenir et la justice des systèmes de retraite, cons. 5 et 2015-715 DC du 5 août 2015, Loi pour la croissance, l'activité et l'égalité des chances économiques, cons. 5. Dans ces deux dernières décisions, le Conseil constitutionnel a été saisi sur le fondement de l'article 61 al. 2 de la Constitution donc dans le cadre de sa compétence de juge de la constitutionnalité des lois. 50 Décision n²015-715 DC, précité, cons. 5. 
terrain d'une éventuelle censure de l'insuffisance de celles-ci, alors même que ces insuffisances sont connues et fréquentes. Le Conseil d'État lui-même souligne les défaillances de l'évaluation préalable et des contrôles exercés ${ }^{51}$ et tire, plus généralement, un «bilan décevant » de l'ensemble des mesures adoptées jusqu’à présent pour simplifier le droit et en améliorer la qualité.

De plus, toutes les lois ne sont pas concernées par ce souci de mieux légiférer. Cela est le cas des lois référendaires qui ne sont pas contrôlées par le Conseil constitutionnel en France, mais c'est également le cas des lois constitutionnelles qui bien qu'assez rares peuvent elles-mêmes être confuses, non normatives, répondant à un effet de mode.

Enfin, dans le cycle de la confection de la loi, la juridiction constitutionnelle n'apparaît que comme l'ultime rouage pour rappeler au législateur ses obligations, avant la promulgation de la loi. La jurisprudence constitutionnelle sur la qualité des lois est d'ailleurs d'une efficacité limitée d'autant que les juges hésitent à prononcer de véritables sanctions. Or, la souplesse du contrôle exercé se justifie à la fois par les difficultés à vérifier le respect de normes dont l'interprétation peut-être très subjective et par la volonté du Conseil constitutionnel de ne pas sortir du rôle qui lui est assigné au risque d'empiéter sur les compétences et les fonctions du gouvernement et du parlement.

Les normes constitutionnelles énoncées par le Conseil constitutionnel lui-même jouent donc avant tout un rôle dissuasif même si elles peuvent parfois donner lieu à des censures ou à des réserves d'interprétation, comme c'est le cas avec l'objectif de valeur constitutionnelle d'accessibilité et d'intelligibilité. Ces censures apparaissent comme l'expression d'une irritation du juge constitutionnel constatant, alors que le processus est achevé, que les dispositions adoptées sont de très mauvaise qualité. Ainsi, dans une comparaison entre les jurisprudences canadienne, espagnole et française sur ce thème, Laurence Gay souligne-t-elle que « la contribution des juridictions constitutionnelles à la lutte contre les malfaçons normatives est nécessairement appelée à être modeste. À l'évidence, l'office du juge s'avère mal adapté pour agir sur les causes profondes de ces malfaçons, qui sont essentiellement politiques. Tout au plus permet-il de veiller à la bonne rédaction du seul texte déféré $»^{52}$.

Mieux légiférer en droit interne est loin d'être un problème qui peut être résolu par le droit constitutionnel ; il s'agit avant tout d'une question politique. En France, il s'agit d'un problème d'une dimension exceptionnellement constitutionnelle pour les raisons qui ont été rappelées ci-dessus mais d'une part, le constituant ne peut pas rigidifier à l'excès la procédure parlementaire en multipliant les contraintes pesant sur le gouvernement et les assemblées dans le but d'améliorer la qualité de la loi, et d'autre part, l'on ne peut pas attendre du Conseil constitutionnel qu'il règle à lui seul des problèmes qui échappent largement à son rôle et aux fonctions habituellement attendues

51 Conseil d'État, Simplification et qualité du droit, Étude annuelle 2016, disponible sur http://www.ladocumentationfrancaise.fr/rapportspublics/164000610-etude-annuelle-2016-du-conseil-d-etat-simplification-et-qualite-du-droit (consulté le 15 mars 2017), p. 77-78.

52 L. GAY, précité, p. 123 
d'une juridiction constitutionnelle. L'amélioration de la qualité des normes est une question qui doit impliquer de nombreux acteurs du Secrétariat général du Gouvernement au Conseil constitutionnel, en passant par les assemblées et le Conseil d'État notamment ; acteurs qui doivent sans doute se responsabiliser davantage, comme le souligne l'étude annuelle 2016 du Conseil d'État ${ }^{53}$, pour tenter d'enrayer le phénomène de dégradation de la qualité de la loi. 



\section{PARTIE II}

\section{Mettre en ceuvre LE « MIEUX LÉGIFÉRER »}





\section{Chapitre 1}

APPROCHES TRANSVERSALES DE LA MISE EN CEUVRE DU « MIEUX LÉGIFÉRER » 



\title{
L'IMPACT DE LA CRÉATION DES ACTES DÉLÉGUÉS SUR LES MÉTHODES D'ÉLABORATION DU DROIT DE L'UNION EUROPÉENNE LE CHEMIN VERS L'ENFER EST PAVÉ DE BONNES INTENTIONS
}

\author{
Laetitia GuILlOUd-COLLIAT ${ }^{1}$
}

\section{Résumé}

Du point de vue de la nomenclature des actes adoptés par les institutions européennes, l'entrée en vigueur du Traité de Lisbonne a conduit à distinguer les actes délégués des actes d'exécution. Si cette réforme a été justifiée par le souci d'améliorer le processus décisionnel de l'Union, sa mise en œuvre a suscité des difficultés et des critiques qui conduisent à douter de l'opportunité de ce changement. En effet, la création des actes délégués a eu pour effet de renforcer la complexité du droit de l'Union européenne d'une part, et d'accroître la nécessité de lutter contre l'opacité de son élaboration d'autre part.

\section{Abstract}

The Lisbon Treaty revised the classification of EU legal acts. More precisely, it distinguished delegated acts from implementing acts. Even if this change sought to improve the effectiveness of EU decision-making, many difficulties and criticisms have emerged in practice, which raises doubts about the impact of this modification. Indeed, the emergence of delegated acts has led to an increase in the complexity of European law as well as the need to fight against its lack of transparency.

Depuis l'entrée en vigueur du Traité de Lisbonne, des actes délégués peuvent être adoptés pour compléter ou modifier certains éléments non essentiels d'un acte législatif ${ }^{2}$. Pour autant, ce procédé n'est pas entièrement nouveau, car dans le cadre des traités antérieurs cette fonction revenait aux actes d'exécution. La Cour de justice considérait en effet qu'un règlement d'exécution pouvait, sous certaines conditions, "fixer des "dérogations" aux dispositions du règlement de base » lorsque ce dernier le prévoyait ${ }^{3}$. Néanmoins, la formalisation au sein des traités européens de la catégorie

\footnotetext{
$1 \quad$ Professeure de droit public, Faculté de droit de Grenoble, CESICE (EA 2420), Université Grenoble Alpes.

2 V. l'article 290 §1 TFUE : «Un acte législatif peut déléguer à la Commission le pouvoir d'adopter des actes non législatifs de portée générale qui complètent ou modifient certains éléments non essentiels de l'acte législatif ».

3 CJCE, 27 septembre 1979, SpA Eridania e.a. contre Ministre de l'agriculture et des forêts e.a., aff. 230/78, Rec. p. 2749 et s. pt 8.
} 
juridique des actes délégués a été justifiée par des préoccupations relatives au " Mieux légiférer » dans l'Union. Comme le rappelait le Comité économique et social européen dans un rapport de 2013 consacré aux actes d'exécution et aux actes délégués : «l'objectif déclaré et assumé du processus qui a conduit à l'adoption des actes délégués était de rendre possible une réglementation plus rapide et souple des aspects des actes législatifs considérés comme étant "non essentiels", tout en garantissant certains mécanismes de contrôle et de suivi. Cet objectif s'inscrit dans la droite ligne des principes du "mieux légiférer" et de la "réglementation intelligente", tels que définis dans de nombreux documents de la Commission, adoptés dans des programmes et des communications de l'UE $»^{4}$.

Les ambitions affichées par les institutions européennes, comme par les États membres, reposaient donc sur l'amélioration de la qualité du droit de l'Union, car en apparence les avantages des actes délégués en la matière sont multiples. Tout d'abord, ils ont vocation à éviter le développement d'une législation trop complexe et/ou trop évolutive, puisqu'ils permettent à la Commission d'adopter les dispositions nécessaires à l'adaptation de celle-ci aux évolutions scientifiques, techniques, conjoncturelles... Ensuite, ils doivent contribuer à accélérer la capacité de réaction normative de l'Union européenne aux changements évoqués ci-dessus, eu égard à la longueur du processus législatif européen qui fait intervenir plusieurs institutions, voire certains organes de l'Union à titre consultatif. Enfin, et en conséquence des arguments précédemment avancés, ils devraient permettre au législateur de se concentrer sur les éléments essentiels de la législation, même s'il n'est pas contraint de se limiter à ces derniers.

Pourtant, des difficultés et des critiques ont émergé à l'occasion de la mise en œuvre de la procédure de délégation ${ }^{5}$. Elles invitent aujourd'hui à se demander si la création des actes délégués permet réellement de «Mieux légiférer » dans l'Union européenne, ou si, dans ce domaine, les intentions - aussi louables soient-elles - n'ont pas finalement conduit à un enfer normatif. En effet, dans son rapport de 2013 déjà évoqué, le Conseil économique et social européen affirmait que « la procédure de délégation doit permettre au législateur de se concentrer sur l'essentiel. On ne peut que s'en féliciter, pour autant que la compétence déléguée à la Commission soit réellement encadrée et mise en œuvre de façon transparente. Après analyse, des doutes demeurent quant à la simplicité de la procédure, la réelle perception par les citoyens européens des enjeux qui sont en cause, l'usage “correct" de cette procédure et l'efficacité des mécanismes de contrôle $»^{6}$.

Le fait est que la catégorie juridique nouvelle que constituent les actes délégués vise « à distinguer entre deux situations qui autrefois se confondaient largement $»^{7}$, celle de l'exécution de l'acte législatif (ou acte de base dans la version antérieure au traité de Lisbonne), et celle de la délégation du pouvoir législatif. Ainsi, dans la pratique, la différenciation des actes délégués et des actes d'exécution, souvent malaisée, a suscité des tensions entre les institutions, lesquelles ont

\footnotetext{
4 CESE, Rapport d'information de la section spécialisée « marché unique, production et consommation » sur le thème " mieux légiférer : actes d'exécution et actes délégués », 30 juillet 2013, Annexe 2, pt 1.1.1.

5 Pour une vue d'ensemble V. C. F. Bergström, D. Ritleng, Rulemaking by the European Commission. The New System for Delegation of Powers. Oxford University Press, 2016.

CESE, Rapport d’information sur le thème « mieux légiférer : actes d'exécution et actes délégués », précité, point 1.11.

J.-P. JAcQué, Droit institutionnel de l'Union européenne. Paris, Dalloz, 2015, p. 510.
} 
conduit au développement d'un important contentieux au sein de l'Union. En outre, alors que les institutions européennes tentent, depuis plusieurs années ${ }^{8}$, d'améliorer la qualité de la législation de l'Union stricto sensu $u^{9}$, les mécanismes d'adoption des actes délégués par la Commission ${ }^{10}$ restent considérés comme opaques, ce qui a conduit cette dernière à développer de nouvelles pistes en la matière. La création des actes délégués a donc eu pour effet de renforcer la complexité du droit de l'Union européenne d'une part (1), ainsi que la nécessité de lutter contre l'opacité de son élaboration d'autre part (2).

\section{1) Le renforcement de la complexité dans l'élaboration du droit de l'Union, ou la difficile délimitation de l'acte délégué et de l'acte d'exécution}

Comme cela a été évoqué précédemment, avant l'entrée en vigueur du Traité de Lisbonne, un acte d'exécution pouvait déroger à un acte de base ${ }^{11}$. Cela est désormais impossible puisque seul l'acte délégué peut, dans les conditions prévues par l'article 290 TFUE, compléter ou modifier l'acte législatif. Ce faisant, le Traité de Lisbonne a imposé une conception de la notion d'exécution plus stricte que celle qui prévalait antérieurement. Or, depuis l'entrée en vigueur du traité, la délimitation de l'acte délégué et de l'acte d'exécution constitue un nouvel objet de contentieux interinstitutionnel.

En effet, pour les institutions détentrices du pouvoir normatif dans l'Union européenne ${ }^{12}$, les enjeux en présence sont considérables ${ }^{13}$. Pour la Commission d'une part, dont les prérogatives varient selon le type de compétence qui lui est octroyé ${ }^{14}$. D’autre part, pour le Parlement européen et le Conseil, dont les pouvoirs en matière de contrôle diffèrent selon la catégorie d'acte qui est adopté. En effet, si l'article 290 § 2 TFUE octroie compétence au Parlement européen et au Conseil pour encadrer la délégation du pouvoir législatif accordée à la Commission, l'article $291 \S 3$ TFUE, pour sa part, confie aux États membres, via les procédures de comitologie, le contrôle de l'exercice de sa compétence d'exécution par la Commission ${ }^{15}$. Le Parlement européen dispose ainsi, sur les actes délégués, de moyens de contrôle dont il est dépourvu à l'égard des actes d'exécution. En revanche, si le Conseil a officiellement perdu le pouvoir d'encadrement qu'il exerçait auparavant sur

\footnotetext{
8 Dès 1992, le Conseil européen d'Édimbourg insistait sur la nécessité de « rendre la nouvelle législation communautaire plus claire et plus simple », et de « rendre la législation existante plus accessible » (Conseil européen d'Édimbourg, 11 et 12 décembre 1992, Conclusions de la présidence, Bulletin des Communautés européennes, $\mathrm{n}^{\circ}$ 12, décembre 1992). Plusieurs accords interinstitutionnels ont ainsi été conclus à partir du milieu des années 1990 et au début des années 2000. V. notamment l'Accord interinstitutionnel du 20 décembre 1994, Méthode de travail accélérée pour la codification officielle des textes législatifs, $7 O C E \mathrm{n}^{\circ} \mathrm{C} 102 \mathrm{du} 4.04$ 1996, p. 2-3; l'Accord interinstitutionnel du 22 décembre 1998 sur les lignes directrices communes relatives à la qualité rédactionnelle de la législation communautaire, fOCE. $\mathrm{n}^{\circ} \mathrm{C} 73$ du 17.03 .1999 , p. 1-4; l'Accord interinstitutionnel du 28 novembre 2001 pour un recours plus structuré à la technique de la refonte des actes juridiques, $7 O C E \mathrm{n}^{\circ} \mathrm{C} 77$ du 28.03.2002, p. 1-3 ; l'Accord interinstitutionnel « Mieux légiférer » du 16 décembre 2003, FOUE n C 321 du 31.12.2003, p. 1-5.

9 C'est-à-dire des actes législatifs selon la nomenclature retenue par le Traité de Lisbonne. V. article 289 TFUE.

10 Lesquels sont, pour leur part, des actes non législatifs. V. article 290 TFUE.

11 V. supra note 3.

12 V. L. Guilloud-Colliat, L'action normative de l'Union européenne. Bruxelles, Bruylant, 2014, $312 \mathrm{p}$.

13 V. G. J. Brandsma, J. Blom-Hansen, « Controlling delegated powers in the post-Lisbon European Union », fournal of European Public Policy, vol. 23, n 4, p. 531-550.

14 Infra.

15 Pour plus de détails sur ce point. V. le règlement $\mathrm{n}^{\circ}$ 182/2011 du Parlement et du Conseil du 16 février 2011 établissant les règles et principes généraux relatifs aux modalités de contrôle par les États membres de l'exercice des compétences d'exécution par la Commission, FOUE, 28.02.2011, pp. 13-18.
} 
la mise en œuvre de sa compétence d'exécution par la Commission ${ }^{16}$, le règlement $\mathrm{n}^{\circ}$ 182/2011 du 16 février $2011^{17}$ prévoit l'intervention d'un comité d'appel, au sein duquel les États membres sont représentés « au niveau approprié ${ }^{18}$, c'est-à-dire éventuellement par leurs ministres, composition qui l'apparente alors au Conseil.

La Cour de justice a ainsi été amenée à se prononcer à plusieurs reprises sur la délimitation des actes délégués et des actes d'exécution, sans véritablement parvenir à convaincre les institutions, ni la doctrine. Contrainte de se livrer à une analyse sémantique de plus en plus approfondie des termes de l'article 290 TFUE, cette dernière a en effet entrepris de distinguer l'action de « compléter » l'acte législatif - par l'intermédiaire d'un acte délégué - de celle visant à " préciser » l'acte législatif par le biais d'un acte d'exécution (1.1). Elle a également été conduite à définir ce que recouvrait la notion de « modification » de l'acte législatif (1.2).

\subsection{L'impossible distinction entre l'action de compléter et celle de préciser l'acte législatif}

Face aux désaccords persistants entre les deux branches du pouvoir législatif, la Commission a invité le Parlement européen et le Conseil, dans une communication du 19 mai 2015, à « s'accorder sur une conception commune révisée des actes délégués, notamment sur les critères qui permettent de les distinguer des actes d'exécution ${ }^{19}$. Néanmoins l'énoncé de critères implique la possibilité de systématiser la distinction entre acte d'exécution et acte délégué. Or, l'idée selon laquelle il serait possible de tracer une ligne de démarcation entre ces deux catégories d'actes, aussi séduisante soit-elle, reste difficile à mettre en ouvre en dépit des efforts entrepris par la Cour de justice en la matière.

Dans un arrêt du 18 mars 2014, la Cour de justice, appelée par la Commission à se prononcer sur l'annulation d'un règlement lui conférant un pouvoir d'exécution plutôt que le pouvoir d'adopter un acte délégué, affirme ainsi que « le législateur de l'Union a pu raisonnablement considérer que l'article 80, paragraphe 1 , du règlement $n^{\circ}$ 528/2012 confère à la Commission le pouvoir non pas de compléter des éléments non essentiels de cet acte législatif, mais de préciser le contenu normatif de celui-ci, conformément à l'article 291, paragraphe 2, TFUE $»^{20}$. La distinction entre l'action de « compléter » et celle de « préciser » l'acte législatif serait donc, aux yeux de la Cour, susceptible de servir de démarcation entre l'acte d'exécution et l'acte délégué. Pourtant, cette distinction reste ténue, d'autant que dans un autre arrêt du 16 juillet 2015, la Haute juridiction s'est opposée à ce que

\footnotetext{
16 En effet, avant l'entrée en vigueur du règlement du 16 février 2011 évoqué supra, dans le cadre des anciennes procédures de comitologie dites de gestion et de réglementation, le Conseil pouvait intervenir en tant qu'instance d'appel, voire se substituer à la Commission. V. Décision 1999/468/CE du 28 juin 1999 fixant les modalités de l'exercice des compétences d'exécution conférées à la Commission, FOCE n L 184 du 17.07.1999, p. 23-26.

17 Précité note 15.

18 Art. 3 \$7 du Règlement n 182/2011 précité.

19 Commission européenne, Améliorer la réglementation pour obtenir de meilleurs résultats - Un enjeu prioritaire pour l'UE, COM (2015) 215 final, 19 mai 2015, p. 10. Les autres apports de cette communication seront évoqués infra.

20 CJUE, gde ch., 18 mars 2014, Commission c./ Parlement et Conseil, aff. C-427/12, point 52. V. Note D. Dero-Bugny, Fournal du droit international, $\mathrm{n}^{\circ}$ 2, 2015, p. 692-696; V. MicheL, « Du départ entre acte délégué et acte d'exécution », Europe, n 5, 2014, comm. 191 ; D. RiTLENG, «The dividing line between delegated and implementing acts : the Court of Justice sidesteps the difficulty, CMLR, vol. 52, n 1, 2015, p. $243-257$.
} 
le pouvoir d'appréciation accordé à la Commission dans l'exercice de sa compétence soit érigé en critère de distinction entre ces deux catégories d'actes ${ }^{21}$. Ce faisant, la Cour de justice a réfuté non seulement la position prise par les avocats généraux - que ce soit dans cette affaire ${ }^{22}$ ou dans celle ayant donné lieu à l'arrêt du 18 mars $2014^{23}$ - mais aussi les déductions doctrinales qui avaient pu être faites à partir de l'analyse de sa jurisprudence antérieure. En effet, dans son arrêt C-427/12 la Cour semblait justifier la légalité de la compétence d'exécution confiée à la Commission par le caractère extrêmement précis de l'acte adopté par le législateur. Certains auteurs en avait alors déduit que « ce qui caractérise [...] l'acte délégué de l'article 290 TFUE par opposition à l'acte d'exécution de l'article 291 TFUE, bien que la Cour se soit refusée à l'expliciter clairement dans l'arrêt Biocides, c'est qu'il est le résultat de l'exercice d'une "large marge d'appréciation" ${ }^{24}$.

La référence au pouvoir d'appréciation de la Commission étant désormais exclue, si l'on s'en tient aux éléments de différenciation retenus par la Cour les actes délégués sont des « règles qui s'insèrent dans le cadre réglementaire tel que défini par l'acte de base ${ }^{25}$, tandis que l'acte d'exécution vise à « préciser le contenu d'un acte législatif, afin d'assurer sa mise en œuvre dans des conditions uniformes dans tous les États membres $»^{26}$. Cette délimitation n'est pas sans soulever certaines difficultés. En effet, « la mise en œuvre ou l'exécution constitue [...] une activité normative, c'està-dire une activité consistant dans l'édiction d'actes juridiquement obligatoires. Il est dès lors très difficile de concevoir un acte d'exécution qui n'ajouterait pas quelque chose au cadre normatif défini par l'acte législatif et, par conséquent, qui ne compléterait pas d'une certaine manière ce dernier [...]. Une mesure qui précise le contenu d'un acte législatif pose, ce faisant, des règles qui complètent le cadre normatif défini par l'acte législatif $\gg^{27}$.

Les limites de cette jurisprudence ont d'ailleurs été récemment mises en évidence dans un arrêt du 17 mars $2016^{28}$. En effet, dans cette affaire, la Cour de justice devait se prononcer sur un règlement législatif, adopté par le Parlement et le Conseil, habilitant la Commission « à adopter des actes délégués [...] précisant les priorités de financement qui devront apparaître dans les programmes de

21 «Contrairement à ce que soutient la Commission, ni l'existence ni l'étendue du pouvoir d'appréciation conféré à celle-ci par l'acte législatif ne sont pertinentes aux fins de déterminer si l'acte à adopter par la Commission relève de l'article 290 TFUE ou de l'article 291 TFUE ». CJUE, gde ch., 16 juillet 2015, Commission c./ Parlement et Conseil, aff. C-88/14, point 32. V. L. Guilloud-Colliat, « L'exégèse des articles 290 TFUE et 291 TFUE par la Cour de justice de l'Union européenne : le juge précise, complète et modifie sa jurisprudence », RAE, $\mathrm{n}^{\circ} 3$, 2015, p. 575-582.

22 V. les conclusions de l'avocat général P. Mengozzi sur l'affaire C-88/14 présentées le 7 mai 2015, point 24 : « la délégation de fonctions législatives implique par nature - du moins, dans la mesure où elle vise à conférer le pouvoir de compléter l'acte de base par des règles de type additionnel - le transfert d'un pouvoir d'appréciation du législateur à l'entité délégataire. Bien que l'article 290 TFUE ne le mentionne pas expressément, le pouvoir d'adopter des actes délégués de nature additionnelle s'accompagne nécessairement de l'exercice d'un certain pouvoir d'appréciation. Il s'ensuit que, lorsqu'un tel pouvoir d'appréciation n'existe pas, l'activité que la Commission est appelée à poursuivre ne se prête pas à être rangée, du point de vue matériel, dans la fonction législative déléguée, mais bien dans la fonction exécutive ».

23 V. les conclusions de l'avocat général P. CRUZ Villalón sur l'affaire C-427/12 présentées le 19 décembre 2013, point 87 : « il suffira d'examiner s'il peut légitimement être soutenu que, dans le cas qui nous occupe, nous nous trouvons déjà au stade de l'exécution, avec les inévitables éléments de spécification ou de détermination finale qui s'imposent, ou si, au contraire, nous nous trouvons encore à un stade dans lequel l'action normative implique des marges d'appréciation dont la Commission ne saurait disposer dans le cadre du seul exercice de son pouvoir d'exécution ».

24 D. RitLENG, «La nouvelle typologie des actes de l'Union. Un premier bilan critique de son application », RTDE, $\mathrm{n}^{\circ} 1,2015, \mathrm{p} .19$.

25 CJUE, gde ch., 16 juillet 2015, Commission c./ Parlement et Conseil, aff. C-88/14, point 29. V. également CJUE, gde ch., 18 mars 2014 , Commission c./ Parlement et Conseil, aff. C-427/12, pt 38.

26 Ibidem, point 30. V. également CJUE, gde ch., 18 mars 2014, Commission c. Parlement et Conseil, aff. C-427/12, pt 39.

27 D. RitLENG, « La nouvelle typologie des actes de l'Union. Un premier bilan critique de son application », précité, p. 17. V. également

L. Guilloud-Colliat, « Actes non législatifs », fcl Europe, fasc. 191-2, mars 2015, § 15.

28 CJUE, 17 mars 2016, Parlement c./ Commission, aff. C-286/14. 
travail $»^{29}$. Plus précisément, la Cour devait ici déterminer si, en utilisant le terme « préciser $»$, le législateur avait entendu autoriser la Commission à compléter ou bien à modifier l'acte législatif en question. La Haute juridiction est alors conduite à affirmer que « la délégation d'un pouvoir de "compléter" un acte législatif ne vise qu'à autoriser la Commission à concrétiser cet acte. Lorsque celle-ci exerce un tel pouvoir, son mandat est limité au développement en détail, dans le respect de l'intégralité de l'acte législatif arrêté par le législateur, des éléments non essentiels de la réglementation concernée que le législateur n'a pas définis $»^{30}$, ce qui revient à assimiler l'action de préciser et celle de compléter l'acte législatif, en contradiction avec sa jurisprudence antérieure ${ }^{31}$.

Les dispositions des articles 290 et 291 TFUE apparaissent ainsi comme une source quasi inépuisable de contentieux. En effet, au-delà de l'impossible distinction entre l'action de préciser et celle de compléter l'acte législatif, la Cour a également été amenée à apprécier la portée d'une «modification » apportée à l'acte législatif, et donc à se prononcer sur la définition qu'il convient de donner au verbe « modifier » tel qu'employé à l'article 290 TFUE.

\subsection{Le débat sur la portée formelle ou substantielle d'une modification d'un acte législatif}

Dans son arrêt du 16 juillet $2015^{32}$, la Cour de justice a ainsi dû déterminer si l'insertion d'une note de bas de page dans un acte législatif constituait ou non une modification de l'acte impliquant l'exercice d'une compétence déléguée ${ }^{33}$. En effet, la Commission s'opposait pour sa part à une conception strictement formaliste de la notion de modification, en affirmant que « même si l'insertion d'une note en bas de page dans un acte législatif constitue, en principe, une modification susceptible de faire l'objet d'un acte délégué, [...] en l'espèce, l'insertion de la note en bas de page prévue par ladite disposition constitue un instrument purement technique utilisé de manière abusive pour travestir l'acte d'exécution en acte délégué ${ }^{34}$. Selon elle, une modification, au sens de l'article 290 $\S 1$ TFUE, devrait avoir « pour effet de changer le contenu normatif de l'acte législatif $»^{35}$. Autrement dit, selon la Commission des modifications mineures, portant sur la forme de texte sans modifier substantiellement son contenu, devraient donner lieu à l'adoption d'un acte d'exécution.

\footnotetext{
$29 \quad$ Ibidem, pt 5.

$30 \quad$ Ibidem, pt 41

31 On remarquera qu'à l'inverse, la Cour de justice accentue la distinction entre les deux catégories d'acte délégué. En effet, elle affirme ainsi que lorsque la Commission est habilitée à compléter l'acte législatif « son mandat est limité au développement en détail, dans le respect de l'intégralité de l'acte législatif [...] des éléments non essentiels [...] que le législateur n'a pas définis » (ibidem, point 41 déjà cité) alors que lorsqu'elle est habilitée à modifier, elle est autorisée à « amender ou à abroger des éléments non essentiels édictés dans cet acte par le législateur [...] [et] elle n'est pas tenue d'agir dans le respect des éléments que le mandat qui lui est accordé vise justement à "modifier" » (ibidem, point 42). En outre, sur le plan formel, l'habilitation à compléter l'acte législatif - contrairement à celle de le modifier - doit nécessairement s'exercer par l'adoption d'un acte distinct de ce dernier. Car, selon la Cour : « la Commission ne saurait, dans le cadre de l'exercice d'un pouvoir de "compléter" un acte législatif, ajouter un élément au texte même de cet acte. En effet, une telle incorporation risquerait de créer une confusion quant au fondement juridique de cet élément, étant donné que le texte même d'un acte législatif contiendrait un élément provenant de l'exercice, par la Commission, d'un pouvoir délégué ne lui permettant pas d'amender ou d'abroger cet acte ». En revanche, "lorsque la Commission "complète" un acte législatif en adoptant un acte distinct, elle peut, dans la mesure nécessaire, modifier ce dernier acte sans être obligée de modifier l'acte législatif même ».

32 Déjà évoqué note 21.

33 Une partie des analyses développées ci-dessous sont également mentionnées in L. GuILLOUD-ColLIAT, «L'exégèse des articles 290 TFUE et 291 TFUE par la Cour de justice de l'Union européenne : le juge précise, complète et modifie sa jurisprudence », précité, p. 580 et $\mathrm{s}$.

34 CJUE, gde ch., 16 juillet 2015, Commission c./ Parlement et Conseil, aff. C-88/14, pt 21.

$35 \quad$ Ibidem, pt 21
} 
Néanmoins, comme le soulignait l'avocat général dans ses conclusions sur cette affaire, la lettre de l'article 290 TFUE « ne fournit [...] aucun point d'appui permettant de faire la distinction entre différentes mesures modificatives de l'acte de base et semble plutôt se fonder sur un critère formel, sur la base duquel, au sens de cette disposition, toute intervention formelle sur le texte de l'acte est une "modification", ce qui a pour conséquence que la fonction de modification relèverait par nature de la délégation législative $»^{36}$. On pouvait néanmoins se demander si « une interprétation aussi large de la notion de modification, qui autorise en substance le législateur à se réserver le contrôle sur des actes matériellement exécutifs, est compatible avec le principe des compétences d'attribution énoncé à l'article 13, paragraphe 2 , TUE, et avec l'équilibre institutionnel voulu par les traités ${ }^{37}$. En effet, comme cela a été évoqué précédemment, les traités prévoient, sur la compétence d'exécution, un mécanisme de contrôle relevant des États membres, dès lors que ces derniers sont les détenteurs par principe de la compétence d'exécution.

La Cour a rendu en définitive un arrêt nuancé. Elle considère en effet que " l'insertion à l'annexe II dudit règlement d'une note en bas de page [...] atteste, ainsi que M. l'avocat général l'a relevé au point 64 de ses conclusions, de la volonté du législateur de l'Union d'insérer l'acte pris sur le fondement de cette disposition dans le texte même du règlement $n^{\circ} 539 / 2001 »^{38}$. Autrement dit, la modification formelle opérée sur le texte est déterminante pour juger de l'effectivité de la modification apportée au règlement. Néanmoins la décision du juge tient également compte des effets de l'acte adopté. En effet, contrairement à l'avocat général qui considérait que la mesure de suspension avait une «nature matériellement exécutive $»^{39}$, la Cour estime que les compétences qui ainsi dévolues à la Commission ont bien « pour effet de modifier, ne fût-ce que temporairement, le contenu normatif de l'acte législatif considéré $\gg^{40}$. Le juge prend donc également en considération les effets de l'acte adopté, ce qui permet d'éviter qu'une conception purement formaliste de la notion de modification ne réduise à néant la compétence d'exécution dévolue à la Commission.

À l'issue de l'examen de cette jurisprudence, on remarquera que, dans le conflit qui oppose les institutions concernant la délimitation entre l'acte délégué et l'acte d'exécution, la Commission, pour sa part, ne privilégie pas systématiquement un type de compétence sur l'autre. En effet, alors que dans l'affaire C-427/12 $2^{41}$ la Commission contestait la décision du législateur de lui confier une compétence d'exécution, en l'espèce elle s'oppose au choix qui a été fait de lui confier le pouvoir d'adopter un acte délégué. Le fait est que si les compétences exercées par la Commission sont plus

\footnotetext{
36 Conclusions de l'avocat général P. Mengozzi sur l'affaire C-88/14 présentées le 7 mai 2015, pt 47.

37 Ibidem, pt 48. Même si l'avocat général se rallie finalement à la conception formaliste : « en conclusion je suis d'avis que nonobstant la nature matériellement exécutive de la mesure de suspension adoptée en vertu de l'article $1 \mathrm{er}$, paragraphe 4 , sous f), du règlement $\mathrm{n}^{\circ} 539 / 2001$, tel que modifié par le règlement litigieux, le recours à l'instrument normatif prévu par l'article 290 TFUE est en l'espèce justifié en raison de la modification que cette mesure apporte à l'acte de base ». Ibidem, pt 67.

$38 \quad$ Ibidem, pt 43. L'avocat général considère en outre que l'article 290 § 1 TFUE qui définit l'acte délégué comme l'acte qui complète ou modifie l'acte législatif distingue deux catégories d'actes délégués : la première « comprendrait les mesures destinées à apporter des modifications formelles au texte de l'acte législatif, la seconde celles qui complètent son contenu normatif sans intervenir sur le texte ». Ibidem, pt 44.

39 Ibidem, pt 66. Plus généralement selon l'avocat général : « les mesures qui prévoient la suspension d'aspects spécifiques du régime légal prévu par un acte législatif, comme les mesures prévoyant sa prolongation ou une dérogation à celui-ci, relèvent, en principe et sans préjudice de la nécessaire appréciation factuelle qui préside à la qualification de la nature des compétences concrètement conférées à la Commission, de la fonction exécutive », pt 58 .

40 CJUE, gde ch., 16 juillet 2015, Commission c. Parlement et Conseil, aff. C-88/14, pt 42.

41 Précitée note 20.
} 
étendues lorsqu'elle agit dans le cadre d'une délégation, le contrôle exercé sur elle par les autres institutions est également plus strict ${ }^{42}$. Ainsi, la Commission considère qu'en prévoyant en l'espèce l'adoption d'un acte délégué dans le cadre de la deuxième phase du mécanisme de réciprocité, le législateur s'octroie en réalité « un droit de veto à [l'encontre d'] une mesure d'exécution $»^{43}$.

On remarquera également que la Cour de justice exerce en définitive un contrôle approfondi sur le choix opéré par le législateur entre acte délégué et acte d'exécution ${ }^{44}$. En effet, si elle reconnait à ce dernier une « marge d'appréciation », c'est dans le strict " respect des conditions prévues par les articles 290 TFUE et 291 TFUE ». Plus précisément, « le pouvoir d'appréciation auquel la Cour fait référence se concrétise dans la possibilité qu'a le législateur de décider selon quelle modalité (acte délégué ou acte d'exécution) l'acte législatif devra être mis en œuvre. Une fois prise cette décision, il appartiendra au législateur d'adapter en conséquence le contenu et la structure de l'acte de base, afin de créer la relation entre les deux niveaux normatifs (acte législatif et mesure de mise en œuvre) se reflétant dans l'une ou l'autre source (acte délégué ou acte d'exécution) ${ }^{45}$. Autrement dit, si le législateur reste maître de l'intensité normative de l'acte (législatif) qu'il édicte - sous réserve d'exercer intégralement sa compétence concernant les « éléments essentiels » de l'acte législatif $^{46}$ - il doit en revanche confier à la Commission la compétence, déléguée ou d'exécution, qui se situe en adéquation avec l'étendue de la compétence législative qu'il a exercée. Un acte législatif incomplet, en ce sens qu'il ne comporte pas l'intégralité des éléments non essentiels de la matière à régir, devra ainsi nécessairement s'accompagner d'une délégation de pouvoir à la Commission sur le fondement de l'article 290 TFUE dès lors que, comme le rappelle ici la Cour, « la Commission en exerçant un pouvoir d'exécution, ne peut modifier ni compléter l'acte législatif, même dans ses éléments non essentiels $»^{47}$. En revanche, si le législateur a épuisé sa compétence législative, et s'il n'entend pas autoriser la Commission à modifier l'acte qu'il a adopté, l'adoption par la Commission d'un acte d'exécution sera seule pertinente, à condition cependant qu'il soit nécessaire d'établir des « conditions uniformes d'exécution » à l'échelle européenne puisque la compétence de principe en matière d'exécution appartient aux États membres ${ }^{48}$. Le contrôle de la Cour de justice sur les modalités d'élaboration des actes législatifs de l'Union européenne a donc ainsi été accru par la formalisation de la distinction entre les actes délégués et les actes d'exécution.

Au-delà du renforcement de la complexité de l'élaboration du droit de l'Union, l'instauration de la catégorie des actes délégués - qui a révélé l'importance du pouvoir normatif exercé par la Commission - a également mis en évidence la nécessité de lutter contre l'opacité de l'élaboration du droit de l'Union, non plus seulement au niveau législatif, mais également à celui des actes non législatifs.

\footnotetext{
V. article 290 §2 TFUE. Voir infra sur la portée de ce contrôle.

CJUE, gde ch., 16 juillet 2015, Commission c./ Parlement et Conseil, aff. C-88/14, pt 25.

Les éléments suivants ont également été développés in L. GuILLOUD-CoLLIAT, « L'exégèse des articles 290 TFUE et 291 TFUE par la Cour de justice de l'Union européenne : le juge précise, complète et modifie sa jurisprudence », précité, p. 578.

45 Conclusions de l'avocat général P. Mengozzi sur l'affaire C-88/14 présentées le 7 mai 2015, pt 36.

46 En effet, en vertu de l'article 290 §1 TFUE, ces éléments ne peuvent faire l'objet d'une délégation à la Commission.

47 CJUE, gde ch., 16 juillet 2015, Commission c./ Parlement et Conseil, aff. C-88/14, pt 31.

48 V. l'article 290 §1 TFUE.
} 


\section{2) La nécessité d'étendre aux actes délégués la lutte contre l'opacité de l'élaboration du droit de l'Union}

Dans un rapport du 29 janvier 2014 consacré à la place des actes délégués dans la législation européenne, la Commission des affaires européennes du Sénat n’hésite pas à évoquer « sa préoccupation sur ce qui a de plus en plus tendance à apparaître comme un transfert de pouvoir, insidieux et mal contrôlé, à la Commission européenne $»^{49}$. En effet, si depuis plusieurs années des efforts ont été entrepris pour améliorer la qualité de l'élaboration des actes législatifs - qu'il s'agisse de l'extension de la procédure de codécision, du développement des études d'impact, de la multiplication des consultations publiques - c'est plus récemment que l'attention de la Commission s'est portée sur la transparence de l'élaboration des actes délégués. Nous aborderons donc successivement les critiques adressées au mécanisme de la délégation (2.1), puis les efforts entrepris pour tenter de remédier à ces difficultés (2.2).

\subsection{Les critiques relatives à l'opacité de l'élaboration des actes délégués}

Si l'article 290 TFUE encadre la procédure d'élaboration des actes délégués, ses dispositions restent assez sommaires et parfois ambigües. Il prévoit en effet, dans son premier paragraphe, que : «Les actes législatifs délimitent explicitement les objectifs, le contenu, la portée et la durée de la délégation de pouvoir. Les éléments essentiels d'un domaine sont réservés à l'acte législatif et ne peuvent donc pas faire l'objet d'une délégation de pouvoir ». Quant au deuxième paragraphe, il ajoute que : «le Parlement européen ou le Conseil peut décider de révoquer la délégation » et que «l'acte délégué ne peut entrer en vigueur que si, dans le délai fixé par l'acte législatif, le Parlement européen ou le Conseil n'exprime pas d'objections ». Certaines dispositions ont ainsi fait l'objet d'interprétations divergentes des institutions, concernant notamment la détermination de la durée de la délégation, ou encore le caractère exhaustif des conditions prévues par cet article ${ }^{50}$.

Des précisions ont, dans un premier temps, été apportées par une « convention d'entente »du 14 avril $2011^{51}$. Elle est notamment à l'origine de l'harmonisation du délai d'objection du Parlement européen et du Conseil à l'égard des actes délégués. Elle précise en effet que ce délai « devrait en principe être d'au moins deux mois, [...] prorogeable de deux mois à l'initiative du Parlement européen ou du Conseil $»^{52}$. Elle prévoit, en outre, que la Commission ne transmet aucun acte délégué entre le 22 décembre et le 6 janvier et entre le 15 juillet et le 20 août ${ }^{53}$, afin que le Parlement européen et le Conseil soient en mesure d'exercer leurs prérogatives de contrôle dans le délai évoqué. En contrepartie, elle instaure une procédure d'urgence, laquelle permet d'échapper à l'effet suspensif

\footnotetext{
49 Rapport d'information fait par M. Simon SuTour au nom de la commission des affaires européennes du Sénat sur la place des actes délégués dans la législation européenne, 29 janvier 2014, p.7.

50 V. notamment G. J. Brandsma et J. Blom-Hansen, « Negotiating the Post-Lisbon Comitology System : Institutional Battles over Delegated Decision-Making », $7 C M S$, vol. 50, n 6, 2012, p. 939-957.

51 Convention d'entente sur les actes délégués, 14 avril 2011 (8753/1/11 REV).

52 Ibidem, pt 10. Rappelons qu'en vertu de l'article 290 § 2 TFUE ce délai est « fixé par l'acte législatif ».

53 Ibidem, pt 6.
} 
de la période d'objection ${ }^{54}$. Cependant, ce délai de deux mois est souvent considéré comme trop court. Dans son rapport d'information du 30 juillet 2013, évoqué ci-dessus, le Conseil économique et social européen affirmait ainsi que l'on pouvait « légitimement se demander si au regard des exigences imposées par le traité, telles que le nombre de voix minimum à atteindre par le Parlement ou l'exigence de la majorité qualifiée au Conseil, les délais relativement courts en pratique (2 mois en général, maximum 4 mois), et la technicité des sujets contenus dans les délégations, le Parlement et le Conseil disposent d'une réelle possibilité d'exercer leur capacité de réviser et de contrôle sur les actes délégués $»^{55}$. Pour autant, la pratique de l'objection n'est pas demeurée strictement formelle ${ }^{56}$.

Une autre critique récurrente porte sur la composition des groupes d'experts assistant la Commission pour l'élaboration des actes délégués. En effet, même si cela n'est pas prévu par l'article 290 TFUE, les actes législatifs autorisant la délégation peuvent prévoir la consultation d'experts par la Commission $^{57}$. Or, si cette consultation peut présenter des avantages en ce sens qu' « elle permet à la Commission de s'assurer que l'acte délégué sera applicable sans difficultés par les administrations nationales compétentes ${ }^{58}$, le flou encadrant la nomination de ces experts est contesté ${ }^{59}$. En effet, il ne s'agit pas nécessairement de représentants des États membres. «Il peut s'agir de professionnels, de scientifiques, d'universitaires, d'experts de quelques États membres seulement, voire, cela n'est pas exclu, d'experts internationaux $\gg^{60}$. Cela nourrit la crainte de renforcement du rôle des lobbys ${ }^{61}$, voire des intérêts étrangers en raison de la présence possible d'experts internationaux.

On observe également que, dans certaines situations, l'intention du législateur a été ignorée voire contournée. Cela s'est notamment manifesté dans le cadre de la réforme de la PAC, dans lequel on a pu observer lors de l'adoption des actes délégués l'adjonction de critères d'éligibilité qui n'étaient pas prévus par l'acte législatif, la réduction du champ de certaines dispositions, la transformation de dispositions facultatives en dispositions obligatoires ${ }^{62} \ldots$ Il est vrai que « l'origine de cette

54 Dans cette hypothèse, en effet, l'acte délégué « entrera en vigueur sans délai et s'appliquera aussi longtemps qu'aucune objection n'aura été émise dans la période prévue par l'acte législatif ». Ibidem, pt 14.

55 CESE, Rapport d'information sur le thème « mieux légiférer : actes d'exécution et actes délégués », précité, Annexe 2, pt 1.3.5. On trouve des préoccupations similaires exprimées dans le Rapport d'information de la commission des affaires européennes du Sénat sur la place des actes délégués dans la législation européenne, précité, p.20.

56 Par exemple le 7 novembre 2014, le Conseil a émis une objection à l'encontre d'un règlement délégué relatif au format de transmission des données sur les dépenses de recherche et développement fondé sur l'habilitation que confère à la Commission l'article 2, paragraphe 5, $\mathrm{du}$ règlement (UE) $\mathrm{n}^{\circ}$ 549/2013 du Parlement européen et du Conseil relatif au système européen des comptes nationaux et régionaux dans l’Union européenne. Un nouveau règlement a finalement été adopté le 30 avril 2015 par la Commission. (Règlement délégué 2015/1365 de la Commission, relatif au format de transmission des données sur les dépenses de recherche et de développement, $7 O U E \mathrm{n}^{\circ} \mathrm{L} 211 \mathrm{du}$ 8.08.2015, p.1). 57 Cette possibilité est mentionnée dans la convention d'entente du 14 avril 2011 précédemment évoquée. V. point $4:$ « Lorsqu'elle prépare et élabore des actes délégués, la Commission veille à ce que les documents pertinents soient transmis simultanément, en temps utile et de façon appropriée au Parlement européen et au Conseil et procède, de manière très anticipée, aux consultations appropriées et transparentes, y compris au niveau des experts ».

58 V. J.-P. JACQUÉ, Droit institutionnel de l'Union européenne, op.cit., p. 512.

59 V. notamment le Rapport d'information de la commission des affaires européennes du Sénat sur la place des actes délégués dans la législation européenne, précité, p. 21-23.

60 Ibidem, p. 21.

61 En effet, comme le souligne le rapport de la commission des affaires européennes du Sénat : « On peut aisément imaginer que lorsqu'il s'agira d'adopter ou de spécifier une norme dans un domaine technique (normes de bruit, normes de pollution, liste des additifs alimentaires, etc...), les experts sollicités - et sélectionnés - seront non seulement de bons connaisseurs des sujets traités mais pourront être sensibles aux préoccupations des milieux industriels impliqués. Sur certains marchés oligopolistiques (automobile, énergie...), les grandes entreprises, même concurrentes, ont un intérêt objectif à définir des règles techniques communes, et à éviter au maximum les spécificités nationales ». Ibidem, p. 22.

62 V. le Rapport d'information de la commission des affaires européennes du Sénat sur la place des actes délégués dans la législation européenne, précité, p. 26-27. 
dérive est bien souvent à chercher dans l'imprécision du texte de base. Le législateur doit veiller à ce que le règlement porteur de la délégation, le texte de base, soit aussi complet que possible et que les éléments essentiels soient précisés. Bien souvent, la Commission ne fait qu'entrer dans une brèche ouverte par le législateur lui-même $»^{63}$.

Enfin, il a également été soulevé à juste titre que le développement de la procédure de délégation s'accompagnait d'un amoindrissement du contrôle des parlements nationaux sur l'élaboration du droit de l'Union. En effet, « La procédure des actes délégués échappe au contrôle de subsidiarité dont disposent les parlements nationaux dans la mesure où ces actes ne sont pas des actes législatifs. Le renforcement des pouvoirs des parlements nationaux dans le contrôle de la législation européenne s'arrête donc au seuil des actes délégués $»^{64}$.

Ainsi, en complément des efforts entrepris pour renforcer la transparence et le caractère démocratique de l'élaboration des actes législatifs, la Commission a tenté de répondre aux critiques concernant les actes délégués.

\subsection{Les efforts entrepris pour remédier à l'opacité des actes délégués}

La Convention d'entente de 2011 n'ayant manifestement pas permis de convaincre pleinement de l'efficacité du contrôle exercé par le Parlement et le Conseil sur la Commission, de nouvelles dispositions ont été prévues, notamment dans l'accord interinstitutionnel du 13 avril 2016 et dans la nouvelle convention d'entente qui l'accompagne en annexe ${ }^{65}$. On peut cependant s'interroger sur le caractère véritablement innovant des solutions avancées. En effet " nonobstant l'insertion de quelques mécanismes additionnels, l'essence même de la boîte à outils du "Mieux légiférer", consistant avant tout en des analyses d'impact et des consultations publiques, n'a pas changé $»^{66}$. Quelques nouveautés méritent néanmoins d'être évoquées, dans l'attente de pouvoir juger concrètement de leur portée.

Dans sa communication du 19 mai 201567, la Commission annonçait ainsi que « pour la première fois, les projets d'actes délégués seront accessibles au grand public pendant quatre semaines sur le site internet de la Commission, parallèlement à la consultation des experts dans les États membres [...]. La Commission publiera en ligne une liste indicative des actes en préparation pour que les parties intéressées puissent s'y prendre en temps utile. Des analyses d'impact proportionnées seront par ailleurs requises pour tous les actes susceptibles d'avoir des effets importants $»^{68}$. La première innovation consiste donc, en matière de consultation, à étendre aux actes délégués ${ }^{69}$ les exigences qui

69 Ainsi qu'aux actes d'exécution puisqu'il est également prévu que « Les actes d'exécution importants qui sont soumis à la comitologie seront eux aussi rendus publics pendant quatre semaines, ce qui permettra aux parties intéressées de formuler leurs observations avant le vote des États membres au sein des comités compétents ». Ibidem, pt 2.1.
} 
étaient jusqu'à présent appliquées aux actes législatifs, sous réserve d'un certain nombre d'exceptions évoquées par la Commission ${ }^{70}$.

Dans le prolongement de cette communication, les institutions ont adopté l'accord interinstitutionnel du 13 avril 2016 " Mieux légiférer». Si certaines de ses dispositions reprennent des engagements déjà inscrits dans l'accord de $2011^{71}$, d'autres comportent certaines avancées. Ainsi, la Commission s'est engagée à procéder à « une analyse d'impact [...] de ses actes délégués et de ses mesures d'exécution qui sont susceptibles d'avoir une incidence économique, environnementale ou sociale importante $\gg^{72}$. La qualité de ces analyses d'impact sera contrôlée par le Comité d'examen de la réglementation, lequel succède au Comité d'analyse d'impact mis en place en $2006^{73}$. Contrairement à ce dernier, le Comité d'examen de la régulation sera composé non seulement de membres internes, mais aussi de membres extérieurs à la Commission en vue d'accroître son indépendance $^{74}$. Néanmoins, comme le souligne Alberto Alemanno, « il reste à voir quel type de relation sera établi entre les membres externes et les membres internes et comment cela affectera l'indépendance générale du comité [...]. On ne peut pas oublier que les membres du CER qui sont fonctionnaires de la Commission (les insiders) vont, à l'issue d'une période de trois ans, retourner dans leur Direction générale d'origine $»^{75}$. Par ailleurs, « la Commission s'engage à rassembler, avant l'adoption d'actes délégués, toutes les connaissances nécessaires, notamment en consultant des experts des États membres et en menant des consultations publiques $\gg^{76}$. Cette disposition vise à remédier aux critiques évoquées ci-dessus et portant sur la composition des groupes d'experts. De plus « afin de garantir l'égalité d'accès à l'ensemble des informations, le Parlement européen et le Conseil reçoivent tous les documents au même moment que les experts des États membres. Les experts du Parlement européen et du Conseil ont systématiquement accès aux réunions des groupes d'experts de la Commission auxquelles les experts des États membres sont invités et qui concernent la préparation d'actes délégués $»^{77}$. Enfin, dans un souci de transparence, les « trois institutions s'engagent à établir, au plus tard pour la fin 2017 et en étroite collaboration, un registre fonctionnel commun des actes délégués $\gg^{78}$ permettant de retracer les différentes étapes de leur élaboration.

70 «Ce sera notamment le cas des projets d'actes d'exécution qui concernent la gestion financière et des projets d'actes délégués ou d'exécution, lorsqu'il n'existe aucune marge de discrétion (ou une marge de discrétion limitée) quant au contenu, lorsqu'une vaste consultation a déjà été organisée au moment de l'élaboration de l'acte (telles celles organisées par les agences de l'UE), lorsque l'urgence empêche l'organisation de consultations ou pour d'autres raisons dûment justifiées ». Ibidem.

71 L'accord de 2016 réaffirme ainsi que le délai d'objection prévu dans l'acte législatif doit être en principe de deux mois, prorogeable de deux mois par chaque institution à son initiative. Il reprend également les périodes durant lesquelles la Commission ne transmet pas d'acte délégué.

72 Accord interinstitutionnel entre le Parlement européen, le Conseil de l'Union européenne et la Commission européenne « Mieux légiférer », 13 avril 2016, précité, pt 13.

73 Pour plus d'éléments sur ce comité et sur l'analyse d'impact en général V. T. DeliLLE, L'analyse d'impact des règlementations dans le droit de l'Union européenne. Paris, Larcier, 2013, 732 p.

74 En outre, les membres internes à la Commission exerceront désormais leurs fonctions à plein temps au sein du comité pendant toute la durée de leur nomination (trois ans non renouvelables). Pour plus d'informations V. la communication de la Commission du 19 mai 2015 «Comité d'examen de la régulation. Mission, tâches et composition, C (2015) 3262 final.

75 A. Alemanno, « Dans quelle mesure le « Mieux légiférer » est-il meilleur ? Une première analyse de l'impact de la nouvelle initiative «Mieux légiférer » sur l’Union européenne », précité, p. 521.

76 Accord interinstitutionnel entre le Parlement européen, le Conseil de l'Union européenne et la Commission européenne «Mieux légiférer», 13 avril 2016, précité, pt 28.

77 Ibidem.

$78 \quad$ Ibidem, point 29. 
Sila pratique permettra d'évaluer la portée de ces avancées, certaines apparaissent déjà critiquables du point de vue de leur efficacité. Ainsi, le développement des consultations devrait entraîner un allongement du processus d'élaboration des actes, alors que la rapidité était l'un des principaux arguments en faveur des actes délégués. Il restera sans doute beaucoup à faire pour parvenir à un bon usage des actes délégués et ainsi convaincre de l'intérêt de l'inscription de cette procédure dans les traités européens. Cependant, le problème n'est pas propre à l'Union européenne ${ }^{79}$. En France, le recours à la législation déléguée, sous la forme des ordonnances, est également considéré par certains comme une cause de « dérèglement juridique et politique $»^{80}$. Dès lors, si pour tenter de remédier aux problèmes constatés, le vice-président du Conseil d'État en appelait à « la science des juristes, à la diligence des pouvoirs publics et à la conscience attentive de nos concitoyens $»^{81}$, les mêmes acteurs pourraient également être sollicités pour participer à la réflexion sur l'encadrement des actes délégués à l'échelle de l'Union.

\footnotetext{
79 V. Ph. Lauvaux, J. Massot, La législation déléguée. Paris, Société de législation comparée, 2014, 166 p. P. Delvolvé, « L'été des ordonnances », RFDA, 2005, p. 909.

81 Intervention de Jean-Marc SAUvé au colloque organisé au Conseil d'État par le Centre d'études constitutionnelles et politiques, l'Institut Cujas et la Société de législation comparée, le 6 juin 2014 sur la «Législation déléguée ». Disponible en ligne sur le site du conseil d’État, http://www.conseil-etat.fr/Actualites/Discours-Interventions/La-legislation-deleguee, p. 11.
} 



\title{
LE « MIEUX LÉGIFÉRER » ET LA LIMITATION DU RECOURS AUX ACTES LÉgISLATIFS EN DROIT DE L'UNION
}

\author{
Stéphane DE LA RosA ${ }^{1}$
}

\section{Résumé}

Les évolutions récentes du « Mieux légiférer » font ressortir, de manière encore plus évidente, un impératif de limitation législative, entendu comme la nécessité non seulement, en amont, de réduire les propositions d'actes législatifs, mais également, en aval, de retirer des propositions pendantes. En outre, cet impératif de limitation emporte des conséquences sur le terrain des équilibres institutionnels, avec en toile de fond un renforcement de l'influence de la Commission sur le déroulement des procédures législatives.

\section{Abstract}

The recent evolutions of "Better regulation " highlight an imperative of legislative limitation, understood as the necessity not only to reduce the proposals of legislative acts, but also, to remove hanging proposals. Besides, this imperative of limitation takes consequences on the ground of the institutional balances, with in the background a strengthening of the influence of the European Commission on the legislative procedures.

Depuis près de deux décennies, l'exigence de «Mieux légiférer » s'est imposée comme un incontournable sur la scène européenne. Bien que de nombreuses études aient déjà été conduites sur la légistique et sur les évolutions qui traversent la production législative en droit de l'Union', l'adoption d'un énième accord interinstitutionnel « Mieux légiférer» le 13 avril 2016³, ainsi que la

Professeur de droit public, Université de Paris Est Créteil, MIL EA 7382.

2 Parmi plusieurs ouvrages: L. Vogel, Better regulation: perspectives critiques, Bruxelles, ETUI Report, 2010, F. PÉRALDI-LENEUf, S. DE LA Rosa (éd.), L’Union européenne et l'idéal de la meilleure législation, Paris, Pedone, coll. Cahiers européens, 2013 ; également les études de R. MeHDI, «L'élaboration des normes communautaires et l'exigence de qualité », in M. FAtin-Rouge, L. GAY, J. Pini (éd.), Autour de la qualité des normes, Bruxelles, Bruylant, coll. À la croisée des droits, p. 177-194 ; O. RENAudie, « Le « mieux légiférer » communautaire. Généalogie et mutation d'un discours institutionnel », in P. MвоNGo (éd.), Le phénomène bureaucratique européen : interprétation européenne et «technophobie », Bruxelles, Bruylant, 2009, p. 111-129.

3 Accord interinstitutionnel entre le Parlement européen, le Conseil de l’Union et la Commission « Mieux légiférer », 13 avril 2016 , fOUE du 12.05.2016, $\mathrm{n}^{\circ} \mathrm{L} 123 / 1$. 
présentation par la Commission d'une nouvelle communication sur ce sujet ${ }^{4}$, justifient de poursuivre les réflexions, de dépasser la permanence des discours pour s'attacher à ce que révèle cet impératif d'une meilleure législation.

Le dernier accord « Mieux légiférer », qui lui-même fait suite à d'autres accords antérieurs portant sur des objets similaires, fait état de la volonté des trois institutions « de poursuivre l'action en vue de mieux légiférer au moyen d'une série d'initiatives et de procédures établies dans le présent accord $»^{5}$. «Poursuivre l'action »: l'expression n'est pas neutre, elle révèle la récurrence d'une problématique inscrite à l'agenda des institutions depuis plus de vingt ans. Un rapide regard rétrospectif permet de prendre la mesure non seulement de la place grandissante de l'exigence de mieux légiférer, mais également de l'extension des significations et des implications qui lui sont associées.

\section{Les différents « temps » du programme «Mieux légiférer»}

Il est possible d'identifier trois temps dans la succession des mesures et des prises de position relatives au « Mieux légiférer».

Dans les années 1990, une série de mesures furent adoptées consécutivement à l'accord interinstitutionnel de 1993 sur les principes de subsidiarité et de proportionnalité6. Il s'agissait de concrétiser les exigences procédurales qui découlent de ces principes, en systématisant la méthode suivie par la Commission pour justifier l'exercice d'une compétence partagée et le contenu de l'acte législatif. De ceci découlent l'attention portée à la motivation de l'acte, le début du recours aux études d'impact pour justifier la valeur ajoutée de l'action communautaire ou encore la justification du degré d'harmonisation retenu, en lien avec le principe de proportionnalité. D’autres considérations ont également été intégrées, telle que la qualité rédactionnelle de la législation, la codification ou encore la refonte des textes législatifs existants ${ }^{7}$. À travers elles, il s'agissait de mettre en œuvre les exigences inhérentes au principe général de sécurité juridique, telles que la lisibilité, l'accessibilité et la clarté de la norme.

À partir du début des années 2000, les orientations et les mesures associées au « Mieux légiférer » s'inscrivent dans une nouvelle orientation, qui fait écho aux débats et aux prises de position sur la nouvelle gouvernance de l'Union. En inscrivant à l'agenda la refonte de la gouvernance de l'Union, expression au demeurant très générale, la Commission, lors de la présentation du Livre blanc sur la gouvernance ${ }^{8}$, a contribué à renouveler la perception de l'action normative de l'Union. À travers la recherche d'une meilleure ou d'une nouvelle gouvernance, il s'est agi d'envisager de nouvelles méthodes de production normative, telles que l'autorégulation, la corégulation, la méthode ouverte de coordination, considérées comme plus souples et moins rigides qu'une méthode communautaire,

\footnotetext{
COM (2016) 615 final du 14.09.2016, Améliorer la réglementation : de meilleurs résultats pour une Union plus forte.

Accord du 13 avril 2016, op. cit., pt. 1.

Accord interinstitutionnel du 25 octobre 1993 sur les procédures pour la mise en œuvre du principe de subsidiarité, $7 O C E, \mathrm{n}^{\circ} \mathrm{C} 329$ du

Accord interinstitutionnel du 22 septembre 1998 sur les lignes directrices communes relatives à la qualité rédactionnelle de la législation communautaire, FOCE 73/1 du 22.12.1998. Cet accord fait suite à l'adoption de la déclaration $\mathrm{n}^{\circ}$ 39, lors du traité d'Amsterdam, relative à la qualité rédactionnelle de la législation communautaire.

8 COM (2001) 428 final, Gouvernance européenne : un livre blanc, 25.07.2001.
} 6.12.1993. 
considérée, avec un excès de simplification, comme le cadre de référence pour concevoir la production des actes législatifs. C'est à cette période que l'expression « Mieux légiférer »se généralise véritablement. L'accord interinstitutionnel « Mieux légiférer » de $2003^{9}$ soulignait en ce sens que les trois institutions « ne légifèrent que dans la mesure nécessaire, conformément au protocole sur l'application des principes de subsidiarité et de proportionnalité. Elles reconnaissent l'utilité de recourir, dans les cas appropriés, lorsque le traité CE n'impose pas spécifiquement le recours à un instrument juridique, à des mécanismes de régulation alternatifs » (pt. 17). Cette mise en avant d'instruments qualifiés d'alternatifs, relativement mal définis ${ }^{10}$, coexiste avec la recherche d'une plus grande qualité de la norme, avec la généralisation de mesures de simplification, telles que la codification, la refonte, ou encore le recours à la législation consolidée. Ainsi que cela a été justement souligné, cet accord de 2003 ne se limite plus à l'amélioration formelle et technique (légistique formelle), il prend également en compte l'ensemble du processus d'édiction des normes et, notamment, leurs effets sur la réalité sociale (légistique matérielle) ${ }^{11}$.

À partir de 2007, le « Mieux légiférer » fait l'objet d'un enrichissement de ses finalités et d'un renouvellement de son utilisation, avec une insistance croissante sur la réduction des charges administratives. Cette évolution est engagée par la présentation, en janvier 2007, d'un programme relatif à la réduction des charges administratives ${ }^{12}$. Le programme part du postulat que la réduction des charges, couvrant les charges administratives liées à la mise en œuvre du droit de l'Union et les coûts techniques associés à la mise en conformité, aura des effets positifs sur l'amélioration de la productivité et de la compétitivité globale ${ }^{13}$. La Commission, avec l'insistance de certains États, se fixe un objectif de diminution de la charge administrative de $25 \%$ et, à cette fin, propose une réorientation de l'évaluation d'impact dans un sens plus favorable aux entreprises, ou encore la multiplication d'une série de structures intermédiaires chargées de passer au crible les initiatives de la Commission ${ }^{14}$. Treize domaines prioritaires sont identifiés pour donner lieu à ce travail de réduction des charges administratives ${ }^{15}$. Ainsi que cela est souligné ci-après, cette orientation du « Mieux légiférer »sera poursuivie et amplifiée avec l'adoption, à partir de 2010, du programme REFIT.

\footnotetext{
$9 \quad$ Accord interinstitutionnel « Mieux légiférer », FOCE C 321/1 du 31.12.2003.

10 Le Livre blanc définissait l'autorégulation comme « un grand nombre de pratiques, de règles communes, de codes de conduite et d'accords volontaires que les acteurs économiques, sociaux, les ONG et les groupes organisés définissent eux-mêmes, sur une base volontaire, pour régir et organiser leurs activités. À la différence de la co-régulation, l'autorégulation n'implique pas nécessairement un acte législatif ». Pour sa part, la co-régulation était définie comme la mise en œuvre « des objectifs définis par le législateur grâce à des mesures arrêtées par les parties actives et reconnues dans le domaine en question ».

11 O. RenAudie, op. cit., p. 122.

12 COM (2007), Programme d'action pour la réduction des charges administratives dans l'Union européenne, 24.01.2007.

13 Dans la présentation de son programme, la Commission avance que si la réduction de $25 \%$ des charges administratives pour les entreprises est effective dès 2012, elle amènerait une augmentation du PIB de 150 milliards d'euros.

14 Certains États, parmi lesquels, le Royaume-Uni, l'Allemagne, les Pays-Bas et le Danemark plaident pour que la réduction des charges administratives devienne un objectif net. V. l'étude très complète de E. VAN DEN ABEELE, « Le programme Mieux légiférer : une nouvelle religion en quête de fidèles ? », in Better Regulation : perspectives critiques, Institut syndical européen, Rapport 113 , p. 59.

15 À savoir, le droit des entreprises, législation pharmaceutique, conditions de travail, droit fiscal, statistiques, agriculture et subventions globales, sécurité alimentaire, transport, pêche, services financiers, environnement, politique de cohésion, marchés publics, in Programme d'action, op. cit
} 


\section{La pluralité de sens du « Mieux légiférer»}

Cette rapide présentation rétrospective du « Mieux légiférer » met en évidence la pluralité des significations qui s'y attache et l'ambivalence de positionnement par rapport à la légistique.

Initialement centrée sur la qualité législative, la simplification, la codification, l'expression «Mieux légiférer » a progressivement faitl'objet d'une montée en généralité. Il est désormais opportun de l'envisager plus comme un concept valise, voire un slogan, a «catchword $»^{16}$, tant il renvoie à des considérations très diverses, liées au déroulement de la procédure législative, à la consultation des parties prenantes, à la pratique des actes délégués par le biais d'une convention d'entente entre les institutions ${ }^{17}$ ou encore à la recherche d'une limitation contre les charges administratives et les lourdeurs, souvent supposées, du droit existant.

Une telle diversité de sens doit être interrogée car elle mobilise deux dimensions distinctes de la légistique. En effet, tel qu'il est présenté par la Commission, le « Mieux légiférer » renvoie, d'une part, à de la légistique formelle, entendue comme l'amélioration rédactionnelle et textuelle, incluant les techniques rédactionnelles destinées à permettre la compréhension de norme énoncée par le texte et, d'autre part, à la légistique matérielle ou substantielle, conçue comme la capacité de la norme à agir sur la réalité, comme les instruments qui permettent de produire le sens de la norme établie par l'énoncé du texte ${ }^{18}$.

\section{Évolutions récentes du «Mieux légiférer »}

Les mesures récentes en faveur du « Mieux légiférer » s'inscrivent tout à fait dans cette dualité de significations. Depuis 2012, la Commission promeut une réglementation « affûtée » (ou fit regulation ), qui entend poursuivre le programme de réduction des charges engagé en 2007. Un programme baptisé « REFIT » (pour Regulatory Fitness and Performance Program) est alors conçu et mis en place afin « de supprimer les coûts inutiles générés par la réglementation (c'est-à-dire les charges) et faire en sorte que l'ensemble de la législation de l'Union reste adaptée à sa finalité ${ }^{19}$. Un tel programme généralise les orientations du « Mieux légiférer » centrées sur la limitation du recours aux actes législatifs et l'appréciation systématique de leurs coûts.

L'accord interinstitutionnel adopté en 2016 amplifie ce mouvement, en envisageant « un rafrâ̂chissement du corpus législatif existant $»^{20}$. Il s'agit là d'une expression très générale, excessivement

\footnotetext{
16 Comme le souligne justemment S. WEATHERILL, « As a slogan 'Better Regulation' invites neither contradiction nor even debate. Who would promote 'Worse Regulation'? Accordingly, the 'Better Regulation» mantra has gathered prolific support in the political debate about the shaping of regulatory environment that is apt to meet the challenges of modern governance", in S. WeATHERILL (éd.), Better regulation, Oxford, Hart publishing, 2007, p. 3

17 V. la contribution de Laetitia GuILloud-Colliat.

18 Voir A. FLÜCKIGER, «Qu'est-ce que «mieux légiférer ? Enjeux et instrumentalisation de la notion de qualité législative », in A. FLÜCKIGER, C. Guy-Ecabert (éd.), Guider les parlements et les gouvernements pour mieux légiférer, Genève, Schulthess, 2008, p. 11-32 ; sur la distinction entre légistique formelle et légistique matérielle, C-A MorAnd, Légistique formelle et matérielle, Presses Universitaires d'Aix-Marseille, 1999. 19 COM (2012) 476 final du 12.12.2012, Pour une réglementation de l'Union bien affutée.

20 L'expression figure dans la communication de la Commission qui a préparé l'accord interinstitutionnel, COM (2015) 215 final, Améliorer la réglementation pour obtenir de meilleurs résultats. Un enjeu prioritaire pour l'Union.
} 
imprécise, qui englobe tout à la fois l'évaluation des charges qui pèsent sur les PME, la planification pluriannuelle des évaluations de la législation et, surtout, l'institutionnalisation d'échanges annuels entre la Commission et les deux colégislateurs que sont le Conseil et le Parlement sur le retrait de propositions pendantes de la Commission, à savoir des propositions en cours de discussion au titre de la procédure législative. Ce sont précisément de telles évolutions qui nous paraissent devoir être interrogées et décryptées. En effet, à côté de considérations relativement classiques, telles l'identification de la bonne base juridique, la simplification du droit existant, la codification, le contenu de l'accord interinstitutionnel et de la communication de la Commission qui lui est consécutive fait ressortir des changements substantiels dans le processus décisionnel. Pour en rendre compte, deux perspectives peuvent être envisagées :

En premier lieu, les évolutions récentes du « Mieux légiférer » font ressortir, de manière encore plus évidente, un impératif de limitation législative, entendu comme la nécessité non seulement, en amont, de réduire les propositions d'actes législatifs, mais également, en aval, de retirer des propositions pendantes (1).

En second lieu, cet impératif de limitation emporte des conséquences sur le terrain des équilibres institutionnels, avec en toile de fond un renforcement de l'influence de la Commission sur le déroulement des procédures législatives (2).

\section{1) Le «Mieux légiférer » et la limitation du périmètre des actes législatifs}

À l'abord, il n'y a rien de véritablement nouveau à ce que la Commission souligne la nécessité de limiter le recours aux actes législatifs à ce qui est essentiel. Ce discours figurait déjà dans le Livre blanc de 1985 sur l'achèvement du marché intérieur ; par ailleurs, les protocoles sur les principes de subsidiarité et de proportionnalité qui se sont succédés ont veillé à systématiser un cadre de justification pour l'édiction des actes de droit dérivée ${ }^{21}$.

Mais les orientations récentes du « Mieux légiférer », dont le Président Jean-Claude Junker a fait siennes dans son discours d'investiture, vont au-delà de cette approche initiale. La limitation du recours aux actes législatifs est envisagée non plus tant comme une donnée à prendre en compte dans l'action normative de l'Union, mais plutôt comme une fin en soi $^{22}$. Réduire le « regulatory burden » est conçu comme allant de pair avec la réforme de la Commission elle-même et, au-delà, de la volonté de rapprocher l'Union de ses citoyens. On peut en prendre la mesure non seulement dans le programme travail de la Commission présenté en 2015, mais également dans les prises de position du premier

21 V. par exemple le protocole $\mathrm{n}^{\circ} 7$ sur l'application des principes de subsidiarité et de proportionnalité résultant du traité d'Amsterdam, dont il ressortait que « la Communauté ne légifère que dans la mesure nécessaire. Toutes choses égales par ailleurs, il convient de donner la préférence à des directives plutôt qu'à des règlements, et à des directives-cadres plutôt qu'à des mesures détaillés [...] en ce qui concerne la nature et la portée de l'action communautaire, les mesures de la Communauté doivent laisser une marge de décision aussi grande que possible au plan national ».

22 Significatif est à cet égard le fait que le programme de travail de la Commission pour 2015 contenait une liste des retraits ou des modifications de propositions en attente, COM (2014) 910 final du 16.12.2014, annexe II. 
vice-Président Timmermans, qui a annoncé, dès la prise de fonction de la nouvelle Commission, sa volonté de renégocier l'accord interinstitutionnel de $2003^{23}$.

Pour concrétiser cette volonté de limiter le recours aux actes législatifs, deux grandes orientations sont suivies ; l'une, qui n'est pas nouvelle, consiste à limiter ex ante l'initiative législative par la densification des études d'impact (1.1), l'autre, plus novatrice, entend organiser le retrait de propositions législatives pendantes (1.2).

\subsection{La limitation par la densification des études d'impact}

Les analyses d'impact sont désormais une étape essentielle du processus de production législative. Elles poursuivent plusieurs finalités : justifier le bien-fondé d'une initiative législative, garantir la transparence de la procédure, mettre en avant un degré souhaitable d'harmonisation (optionnel, minimal, maximal), légitimer la norme à venir par l'accueil a priori positif de ses destinataires. Cette diversité de considérations justifie que la pratique des études d'impact se soit progressivement amplifiée et complexifiée ${ }^{24}$. Plus largement, les études d'impact ne servent pas seulement à justifier une proposition législative, mais également à prendre la mesure d'une réception favorable par le Conseil et le Parlement ${ }^{25}$.

Dans la nouvelle mouture du programme « Mieux légiférer », les études d'impact connaissent deux évolutions significatives, qui vont dans le sens de l'élargissement des critères d'appréciation qu'elles mobilisent et de l'accentuation des contraintes de justification avant la proposition législative.

En premier lieu, l'accord interinstitutionnel de 2016 et la communication présentée en septembre de la même année envisagent un accroissement des critères qui doivent être pris en compte dans les analyses d'impact. En l'état actuel, celles-ci sont élaborées par les services de la Commission (concrètement par la DG qui pilote une proposition législative) au regard de lignes directrices adoptées en $2009^{26}$. Le cadre de confection qui résulte des lignes directrices est d'ores et déjà passablement complexe. Pas moins de six étapes, correspondant chacune à plusieurs critères ${ }^{27}$, doivent être développées dans la réalisation de l'étude d'impact, laquelle incombe en premier lieu à la direction chef de file (à savoir la direction porteuse du projet d'acte) au sein de la DG de la Commission compétente. Une étude

\footnotetext{
23 V. le discours prononcé par le vice président le 19 mai 2015 : «La Commission est déterminée à modifier à la fois ce que fait l'Union et la façon dont elle le fait. L'amélioration de la réglementation est dès lors l'une de nos priorités essentielles. Nous sommes à l'écoute des préoccupations des citoyens et des entreprises - en particulier des PME - qui s'inquiètent de ce que Bruxelles et ses institutions établissent des règles qu'ils ne sont pas toujours en mesure de comprendre ou d'appliquer. Nous voulons restaurer leur confiance dans la capacité de l'UE à élaborer une législation de qualité. Il ne s'agit pas d'augmenter ou de réduire la quantité de règles de l'UE, ni de revoir à la baisse nos normes sociales et environnementales, de compromettre notre santé ou de mettre en péril nos droits fondamentaux. Il s'agit de veiller à réaliser de la manière la plus efficiente possible les objectifs ambitieux que nous nous sommes fixés », disponible sur http://europa.eu/rapid/press-release_IP-15-4988_fr.htm. 24 Th. Delille, L'analyse d'impact des règlementations dans le droit de l'Union européenne, Bruxelles, Larcier, coll. Faculté du Luxembourg, 2013. 25 Ce phénomène d'extension des critères entrant en ligne de compte dans l'analyse d'impact est parfaitement mis en lumière dans l'étude d'Alberto Alemanno, "Quis Custodet Custodes dans le cadre de l'initiative "Mieux légiférer" ? : une analyse des mécanismes de surveillance de la qualité réglementaire au sein de la Commission européenne et la création du Comité d'évaluation des analyses d'impact », Revue du droit de l'Union européenne, 2008, $\mathrm{n}^{\circ}$ 1, p. 43-86, spec. p. 51.

26 Commission européenne, Impact Assessment Guidelines, 15 janvier 2009, SEC (2009) 92. Ces lignes directrices élargissent celles définies dans COM (2002) 276 final, Communication de la Commission sur l'analyse d'impact.

27 Les lignes directrices identifient six «key analytical steps», comprenant : "identifying the problem", "define the objectives", "develop main policy options", "analyse the impacts of the options", "compare the options", "outline policy monitoring and evaluation", SEC (2009) 92, op. cit.
} 
approfondie des lignes directrices souligne la faible marge de manœuvre dont disposent les agents de la Commission pour réaliser les études d'impact : l'appréciation des impacts économiques, sociaux et environnementaux d'une législation envisagée doit être passée au crible de tout un ensemble de questions et de critères préalablement définis. Pareille formalisation présente deux inconvénients majeurs ; d'une part, elle conduit à transformer l'analyse d'impact en un exercice de style qui obéit à des règles pré-définies, d'autre part, elle favorise le recours à des expertises extérieures, réalisées par des consultants indépendants, lorsqu'il s'avère que la matière est excessivement complexe.

Dans les orientations récentes du « Mieux légiférer », la Commission propose une révision de ces lignes directrices et l'institutionnalisation d'un comité, au sein de la Commission, pour évaluer leur qualité. Telle est désormais la fonction du « Regulatory Scrutiny Board», institué en $2015^{28}$, qui fait suite au comité interne d'études d'impact. Sa mise en place a pour le moins été discrète, puisqu'elle résulte d'une décision du Président de la Commission prise sur le fondement du règlement intérieur de celle-ci. Son champ d'intervention est en revanche relativement étendu, dans la mesure où il apprécie l'ébauche d'étude d'impact réalisée par la DG tête de file d'une proposition législation au regard de tout un ensemble de critères : standards des études d'impact, exigence de consultation, «fitness check!»; bien au-delà des seules exigences formelles d'adoption de l'acte ${ }^{29}$. En accroissant les contraintes d'évaluation en amont de la proposition législative, l'institution d'un comité de ce type permet de justifier une forme d'autolimitation de l'initiative, au demeurant voulue, de la Commission et de présenter des propositions déjà « évaluées » au regard d'exigences et de critères qui devraient être pris en compte par les deux co-législateurs.

En second lieu, outre la densification des études d'impact préalables aux propositions législatives, les évolutions récentes du « Mieux légiférer » envisagent également la possibilité pour le Parlement et le Conseil de procéder à des modifications substantielles des analyses menées par la Commission. En ce sens, l'accord « Mieux légiférer» de 2016 souligne que « lorsqu'ils le jugeront approprié et nécessaire aux fins du processus législatif, le Parlement européen et le Conseil effectueront des analyses d'impact des modifications substantielles qu'ils apportent à la proposition de la Commission. En règle générale, le Parlement européen et le Conseil prendront comme point de départ de leurs travaux complémentaires l'analyse d'impact de la Commission. Il appartient à chaque institution concernée de déterminer ce qui constitue une modification substantielle ». Cette possibilité de modification doit se faire en veillant à la cohérence du travail d'analyse d'impact « dans son ensemble $»^{30}$. S'il est encore trop tôt pour apprécier l'effectivité de telles études d'impact «modificatives », il est certain qu'elles auront pour conséquence d'ajouter encore de la complexité au processus législatif.

28 C (2015) 3263 final du 19.05.2015, Décision of the President of the European Commission on the establishment of an independent Regulatory Scrutiny Board. Notons que ce comité est présenté comme indépendant mais administrativement rattaché au secrétariat général de la Commission. V. la présentation sur le lien : https://ec.europa.eu/info/law/law-making-process/regulatory-scrutiny-board_en\#documents

29 V. ainsi les règles de procédures définies par le Scrutiny Board, ref. ARES (2016) 5764766, 5.10.2016, art. 6 : "The Board shall assess the quality of draft impact assessment reports, fitness check reports and major evaluation reports [...] it shall deliver its opinions, recommendations and advice in accordance with the Commission's policy on better regulation, as laid down in the Better Regulation Guidelines, and other relevant instructions to the services on agreed standards for impact assessment, evaluation, fitness checks and public consultation".

30 Accord interinstitutionnel du 13 avril 2016, pt. 15 et 16. 


\subsection{La généralisation du retrait de propositions pendantes}

En lien avec le programme REFIT, une nouvelle orientation du « Mieux légiférer » - sûrement la plus significative - consiste à encourager l'abrogation de législations qui seraient obsolètes ou qui seraient excessivement contraignantes. En ce sens, l'accord interinstitutionnel de 2016 prévoit explicitement que le programme de travail de la Commission doit comporter une liste d'abrogations. Il y est par ailleurs précisé que, « conformément aux principes de coopération loyale et d'équilibre institutionnel, si la Commission prévoit de retirer une proposition législative, qu'il soit ou non envisagé de la remplacer par une proposition révisée à la suite du retrait, elle communiquera les raisons de ce retrait et, le cas échéant, indiquera les étapes ultérieures prévues ainsi qu'un calendrier précis, et elle mènera des consultations interinstitutionnelles appropriées sur cette base $»^{31}$.

Avant même que l'accord ne procède à la systématisation du retrait de propositions pendantes, la Commission, dans son programme de 2015, a présenté une typologie des différents cas de retrait. Elles sont justifiées par le défaut d'utilité de la proposition, par l'enlisement des négociations entre le Conseil et le Parlement, ou encore par obsolescence. Dans cette perspective, les programmes de travail adoptés pour 2015, 2016 et 2017 ont chacun envisagé une série de retraits (respectivement, 80, 28 et 19 retraits). Peuvent être distingués :

- d'un côté, des retraits justifiés par l'obsolescence de la proposition, les plus nombreux, qui correspondent à des propositions d'actes qui sont retirées en raison de leur caducité du fait de l'entrée en vigueur d'un autre acte avant un objet similaire. Pour 2017, les 19 retraits sont ainsi justifiés sur le terrain de l'obsolescence. Cette hypothèse se vérifie pour des mesures liées à la PAC, à l'environnement, voire pour certains accords d'association. Si ces retraits sont justifiables d'un point de vue pratique, ils peuvent également marquer l'abandon d'une proposition cohérente et lisible par rapport à son objet ${ }^{32}$.

- d'un autre côté, des retraits de proposition législation qui reposent sur des considérations d'opportunité, pour trois motifs principaux :

- L'absence d'un accord prévisible entre les deux co-législateurs, dans des matières très diverses, par exemple une proposition sur l'enregistrement des transporteurs de matières radioactives, une proposition sur les taxes des voitures particulières, une proposition sur la fixation des prix des médicaments et leur inclusion dans les systèmes publics d'assurance maladie, ou encore la proposition de règlement du Conseil relatif au statut de la fondation européenne ${ }^{33}$. Dans ce cas de figure, c'est l'enlisement de la proposition, pendant parfois depuis plus de 10 ans, comme par exemple la proposition de règlement sur la fiscalité des véhicules à moteur, qui remonte à 1998. Mais le recours à cette justification par la Commission peut prêter

\footnotetext{
31 Op. cit., pt. 9.

32 Par exemple, l'annonce du retrait en 2017 d'une proposition de règlement sur les substances psychoactives, justifiée par le fait que certains éléments de celle-ci ont été repris dans plusieurs textes....qui lui sont antérieurs ! (notamment la décision cadre du Conseil du 25 octobre 2004 sur l'harmonisation des infractions pénales dans le domaine du trafic de drogue).

33 Voir les propositions de retrait qui figuraient dans le programme de travail de la Commission de 2015, COM (2014) 910 final du 16.12.2014.
} 
à discussion, notamment lorsque l'argument temporel est combiné à des considérations telles que le risque de dénaturation de la proposition de la Commission, pourtant déjà examinée par le Conseil (argument pour justifier le retrait d'une proposition de directive (COM 2011, 0169) relative à la taxation des produits énergétiques de l'électricité).

- La nécessité d'introduire une proposition qui serait plus ambitieuse que celle qui est pendante. C'est sur ce terrain que la Commission justifie, dans son programme de travail de 2017, le retrait d'une proposition de directive concernant une assiette commune consolidée pour l'impôt sur les sociétés, censée être remplacée par une approche plus progressive qui privilégie une assiette fiscale commune obligatoire.

- Lamise enœuvre du programmeREFIT, quijustifierait le retrait de propositions excessivement lourdes et associées à des coûts administratifs jugés excessifs. Peu étayé dans les documents de la Commission, ce fondement permet de justifier le retrait de propositions symboliquement importantes, telles la proposition de directive sur la santé et la sécurité des femmes enceintes ou encore la proposition d'un fonds d'indemnisation pour les dommages causés par la pollution par les hydrocarbures ${ }^{34}$.

En tout état de cause, ces annonces de retrait, outre le fait qu'elles reflètent une extension des conceptions associées au "Mieux légiférer », emportent des conséquences tangibles à l'échelle institutionnelle.

\section{2) Le « Mieux légiférer » et la reconfiguration des équilibres institutionnels}

Depuis plus de deux décennies, il est courant de constater que le pouvoir d'initiative de la Commission fasse l'objet d'un affaiblissement substantiel ${ }^{35}$. Ce pouvoir est, au plan des principes, consacré par le traité, d'une part, par l'article 289 TFUE, qui prévoit que le Conseil et le Parlement statuent sur proposition de la Commission, d'autre part, par l'article 293 TFUE qui reconnait que ladite proposition peut être modifiée tant que le Conseil ne l'a pas, le cas échéant, modifiée à l'unanimité. Cette dernière disposition permet de garantir que le texte examiné et soumis à la discussion est, presque systématiquement, celui de la Commission.

Sous l'influence de plusieurs facteurs, tels que l'accroissement du rôle du Conseil européen, le transfert de nouvelles compétences à des agences, le besoin croissant d'appuis techniques et opérationnels plutôt que de normes législatives (par exemple dans le champ de l'ELSJ), l'adoption de cadres d'orientation sous forme de cycles d'évaluation (par exemple le semestre européen dans le champ de la gouvernance économique) ou encore la complexification de la hiérarchie du droit dérivé avec les actes délégués, le schéma traditionnel d'une Commission dont l'activité se concentrerait sur l'élaboration des propositions législatives est sensiblement remis en cause. La réalité d'une initiative 
véritablement autonome de la Commission peut également être discutée, dès lors que la quasi-totalité des propositions sont issues d'engagements internationaux ratifiés par l'Union ou de demandes d'États membres. À travers l'évolution de son rôle, ce sont certaines conceptualisations classiques de l'intégration européenne (on pense par exemple à la méthode communautaire) qui ne sont plus systématiquement opératoires.

Néanmoins, la place croissante du « Mieux légiférer » conduit à proposer une autre lecture de ces évolutions. Les différentes dimensions de ce programme sont peut-être plus illustratives d'un « changement de paradigme » du rôle de la Commission que l'expression d'un déclin trop rapidement constaté $^{36}$. L'ensemble des mesures et des instruments associés au «Mieux légiférer » est plutôt significatif d'un renforcement du contrôle exercé par la Commission (2.1) et d'une complexification de la procédure législative (2.2).

\subsection{Le renforcement du contrôle de la Commission par la pratique du retrait}

Une fois que la proposition législative est présentée devant le Conseil et le Parlement, la Commission exerce essentiellement une mission d'intermédiaire, "d'honnête courtier », pour rapprocher la position des deux co-législateurs ${ }^{37}$. Cette position repose sur une double justification, d'une part, éviter que la négociation ne conduise à dénaturer sensiblement la proposition initiale, d'autre part, permettre un accord rapide, de préférence en première lecture.

Or, la pratique du retrait, telle qu'elle a été présentée ci-dessus, conduit à relativiser ce schéma traditionnel. Elle conduit, notamment, à s'interroger sur la portée et sur le respect du principe de l'équilibre institutionnel, notamment lorsque le retrait intervient des années après le dépôt de la proposition ou après que le Conseil et le Parlement aient déjà eu des échanges de vues. L'équilibre institutionnel étant intrinsèquement lié à une exigence de coopération loyale entre les institutions $\mathrm{s}^{38}$, le recours non encadré au retrait des propositions par la Commission peut mal s'accommoder avec celle-ci.

Il ne s'agit pas là d'une hypothèse d'école, dès lors que cette problématique a été au centre de l'affaire dite de l'assistance macrofinancière, jugée par la Cour le 14 avril $2015^{39}$. Inédite, l'espèce met en cause le retrait par la Commission d'une proposition de règlement relative à l'assistance macrofinancière aux pays tiers, à savoir un instrument par lequel l'Union peut octroyer une aide exceptionnelle pour rétablir la viabilité des finances extérieures de pays confrontés à des difficultés de financement extérieur. Pour justifier son retrait, la Commission avance ici la dénaturation de la

36 V. l'analyse pertinente que propose J.-P. JACQUÉ, « La Commission européenne après Lisbonne: déclin ou changement de paradigme », in Liber Amicorum en l'honneur du professeur Vlad Constantinesco, Bruylant, 2015, p. 241-266.

37 La qualification de « courtier » ou « honest broker» a pu être retenue dans certains documents institutionnels, par ex. Déclaration commune du Parlement européen, du Conseil et de la Commission sur les modalités pratiques de la procédure de codécision (article 251 du traité CE), fOCE, 2007, ${ }^{\circ}$ C $145 / 5$.

38 Cette imbrication est régulièrement rappelée dans la jurisprudence, v. par exemple le contentieux sur l'intervention de l'Union devant le Tribunal international du droit de la mer, CJUE, 6 octobre 2015, Conseil c./ Conseil, aff. C-73/14, pt. 84.

39 CJUE, gr. ch., 14 avril 2015, Conseil de l'Union c./ Commission, aff. C-409/13, ECLI:EU:C:2015:217. 
proposition, dans la mesure où le Parlement et le Conseil l'ont modifiée pour prévoir que la décision d'assistance doit, au cas par cas, suivre la procédure législative ordinaire et non une procédure d'exécution proposée par la Commission. Pour appuyer son recours en annulation, le Conseil, appuyé par dix États membres, invoque en l'occurrence le principe de l'équilibre institutionnel, inhérent à l'article 13 du TUE et le principe de démocratie, énoncé à l'article 10 TUE.

L'argumentation suivie par la Cour est intéressante à plusieurs titres. Elle considère que si la Commission, en vertu même du traité, a le pouvoir de modifier voire de retirer sa proposition tant que le Conseil n'a pas formellement statué (ce qui était le cas ici) ; en revanche, l'exercice de ce droit de retrait ne peut conduire à lui conférer un droit de veto dans le déroulement du processus législatif ${ }^{40}$. Lorsque la Commission, après avoir présenté une proposition dans le cadre de la procédure législative ordinaire, décide de la retirer, elle doit exposer au Parlement et au Conseil les motifs du retrait. En cas de contestation, ceux-ci doivent être étayés par des éléments convaincants. La décision de retrait est dès lors conçue comme un acte faisant grief, susceptible d'un recours en annulation et tenu à une obligation générale de motivation.

Cette qualification est importante car elle conduit à déplacer le terrain contentieux : la question n'est pas tant celle de savoir si la Commission peut retirer, mais plutôt comment elle justifie le retrait. Pour la Cour, la dénaturation d'une proposition par les co-législateurs est un motif suffisant pour fonder une telle justification. La dénaturation ne peut être unilatéralement appréciée par la Commission, dès lors que «dans l'esprit de coopération loyale qui, en vertu de l'article 13, paragraphe 2, TUE, doit présider aux relations entre institutions de l'Union dans le cadre de la procédure législative ordinaire, elle doit dûment prendre en compte les préoccupations du Parlement et du Conseil sousjacentes à leur volonté d'amender $»^{41}$.

Bien que cette affaire n'ait pas été liée à un retrait justifié par le programme « Mieux légiférer », des liens peuvent être établis entre l'argumentation de la Cour et la pratique du retrait telle que présentée ci-dessus. On peut se demander quelle pourrait être la position du juge vis-à-vis de motifs de retrait distincts de la dénaturation. Par exemple, peut-on considérer que le risque d'un défaut d'accord entre le Conseil et le Parlement, qui a justifié le retrait par la Commission de plusieurs propositions (par exemple la directive sur les travailleuses enceintes ou le règlement sur la fondation européenne) peut valablement motiver un retrait ? À l'évidence, le motif du retrait sera plus délicat à apprécier dès lors qu'il renvoie à une appréciation subjective de la négociation (le défaut d'accord, nécessairement changeant au regard de la composition du Conseil et du Parlement) et non à une donnée plus objective (une différence substantielle entre contenu de l'acte amendé par rapport à celui qui était proposé).

$40 \quad$ Aff. C-409/13, op. cit., pt. 75 : « Le pouvoir de retrait que la Commission tire des dispositions mentionnées [art. $17 \S 2$ TUE, art. 289 et 293 TFUE] ne saurait toutefois investir cette institution d'un droit de véto dans le déroulement du processus législatif, qui serait contraire aux principes d'attribution de compétences et de l'équilibre institutionnel ».

41 Op. cit., pt. 83. 
De manière plus fondamentale, il est nécessaire de s'interroger sur les interférences entre la pratique du retrait et le principe de démocratie. Il s'agit sûrement là d'une faiblesse du raisonnement de la Cour, justement souligné par Dominique Ritleng ${ }^{42}$. L'arrêt écarte trop rapidement le moyen tiré du principe de démocratie, pour l'associer à celui de l'équilibre institutionnel. De jurisprudence constante, le principe de démocratie implique, en droit de l'Union, qu'une proposition soit dûment examinée par l'intermédiaire d'une assemblée représentative ${ }^{43}$. Ainsi que l'a souligné l'Avocat général Poiares Maduro, ce principe renvoie, à l'échelle de l'Union, à un équilibre délicat entre les dimensions nationales et européennes. Nécessairement imbriquées, elles conduisent à ce que le processus législatif soit partagé entre des parlements, nationaux et européen, qui ne disposent pas du même pouvoir ${ }^{44}$. La Cour, dans l'affaire en cause, ne fait pourtant guère de cas de ces exigences, pour privilégier une lecture trop classique des rapports entre les institutions et maintenir l'image, pourtant passablement écornée, d'un triangle institutionnel parfaitement représentatif des exigences démocratiques. La confrontation des pratiques associées au « Mieux légiférer » avec celles-ci ne peut être durablement évitée ${ }^{45}$, au risque de conforter la perception, déjà répandue, d'une conception par trop financière et utilitariste de la légistique.

\subsection{La complexification de la procédure législative}

Outre ses conséquences sur le terrain de l'équilibre institutionnel, la pratique du retrait conduit également à s'interroger sur le déroulement de la procédure législative. De fait, le discours sur le «Mieux légiférer» va de pair avec une recherche de rapidité de la procédure. Loin de la représentation selon laquelle l'acte législatif serait le fruit de trois lectures consécutives entre le Parlement et le Conseil (prévues à l'article 294 TFUE), la pratique des négociations directes entre le Parlement européen et le Conseil, dès les premières étapes de la procédure, a redéfini le rôle de la Commission. $\mathrm{Au}$ lieu de chercher à amender sa proposition auprès une première lecture, la Commission cherche à intervenir dès les premiers stades de l'examen.

Désormais, plus de $80 \%$ des accords sur les propositions législatives s'établissent dans le cadre de réunions qui prennent la forme de trilogue, lesquelles interviennent, après l'appréciation de la proposition par la commission parlementaire compétente, entre l'adoption de la résolution législative en plénière du Parlement et le vote du Conseil ${ }^{46}$. Ces trilogues se tiennent pour l'essentiel à huis clos, hors du débat public. L'accroissement du recours à ces instances informelles est susceptible de justifier, aux yeux de la Commission, les orientations récentes du programme « Mieux légiférer ». Le retrait de la proposition peut, en effet, être envisagé comme une carte à jouer par la Commission dans les négociations, lorsqu'au sein du trilogue leur déroulement fait craindre une dénaturation

42 D. Ritleng, «Does the European Court of Justice take democraty seriously? Some thoughts about the Macro-Financial Assistance case», CMLR, vol. 53, février 2016, p. 11-34.

43 Formulation issue de la célèbre jurisprudence Roquette(C-138/79) et rappelée plus récemment dans les contentieux relatifs à la participation du Parlement dans les mesures prises au titre de la PESC, CJUE, gr. ch., 19 juillet 2012, Parlement c./ Conseil, ECLI:EU:C:2012:472, pt. 81.

44 V. les conclusions de l'avocat général MADURO dans l'affaire dite du transfert de déchets, C-411/06, pt. 5.

45 F. Peraldi Leneuf, «Légistique et démocratie dans le système de l’Union européenne », in F. Peraldi Leneuf (éd.), La légistique dans le système de l'Union européenne. Quelle nouvelle approche ?, Bruxelles, Bruylant, 2012, p. 111-125.

46 J.-P. JACQUÉ, op. cit. 
excessive de celle-ci. En avançant le risque de retrait, la Commission exerce plus qu'une simple mission d'intermédiaire pour rapprocher les positions des législateurs.

Au-delà du retrait, on peut également s'interroger sur la portée des évaluations réalisées avant la proposition par la Commission (notamment les études d'impact) sur le déroulement de la procédure législative. Du fait de contraintes sans cesse croissantes de célérité et de réactivité, l'adoption des actes législatifs est menée suivant un calendrier plus serré qu'auparavant. Dans ce contexte, les appréciations techniques qui ont été menées par la Commission dans ses études d'impact, de même que l'appréciation des coûts administratifs de la proposition au titre du programme REFIT, peuvent être avancées comme des arguments d'autorité, présentées telle une vérité scientifique, durant le déroulement des négociations et la tenue des trilogues. Il y a toutefois là un risque de glissement dans la conception qui s'attache à la légitimité de l'acte. La mise en avant d'un discours sur les charges et les coûts générés par la proposition peut conduire à deux conséquences négatives, d'une part une mise à l'écart de débats de fond sur les options politiques à privilégier en vue de satisfaire l'intérêt général, d'autre part, une orientation du pouvoir d'amendement sur les dimensions financières de la proposition.

Le risque tient également à la multiplication des critères qui interviennent dans l'élaboration de l'acte législatif. Aux critères strictement juridiques, liés à la base juridique, à la formulation de l'acte, à sa motivation, s'ajoutent des considérations tirées de ses coûts et de sa capacité à réduire les charges administratives, le « regulatory burden ». À travers ces évolutions, se dessine un glissement de la négociation, qui ferait prévaloir des appréciations proprement techniques et financières, symptomatiques d'une gouvernance par les nombres ${ }^{47}$, et non une discussion des conceptions de l'intérêt général qui s'attachent à l'acte soumis à la délibération.

47 Selon le titre retenu par Alain Supiot pour ces cours au Collège de France, A. Supiot, La Gouvernance par les nombres, Paris, Fayard, 2015. 



\title{
La Cour de JUSTice de L'Union européenNe ET LE « MIEUX LÉGIFÉ RER » RECHERCHE SUR L'IMPACT JURIDICTIONNEL
}

\author{
Nathalie RUBIO ${ }^{1}$
}

\section{Résumé}

Cette étude a pour objet le positionnement de l'organe juridictionnel dans la gouvernance européenne et la visibilité de sa contribution à la mise en œuvre des exigences du « Mieux légiférer ». Il s'agit en effet de déterminer l'impact du juge mais aussi l'impact sur le juge. La recherche est menée tant d'un point de vue matériel au regard de l'analyse de la jurisprudence que d'un point de vue formel au regard des méthodes et outils utilisés par le juge pour appliquer et interpréter le droit de l'Union européenne. Des pistes sont explorées quant aux possibilités de lui reconnaître un rôle plus ambitieux dans la fabrication du droit de l'Union européenne.

\section{Abstract}

The object of this study is the position of the judge in the European governance and the visibility of its contribution to the implementation of the Better regulation texts. It is indeed a question of determining the impact of the judge but also the impact on the judge. The search is led so much material point of view with regard to the analysis of the jurisprudence that from a formal point of view with regard to the methods and the tools used by the judge to apply and interpret European law. The principal question is knowing if there are any possibilities of recognizing a more ambitious role for him in the UE decision-making.

1 Professeure de droit public, Chaire Jean Monnet, Aix Marseille Univ, Université de Toulon, Univ Pau \& Pays Adour, CNRS, DICE, CERIC, Aix-en-Provence, France. 
Comment la Cour de justice de l'Union européenne peut-elle être un « acteur » du « Mieux légiférer $»^{2}$ ? La question peut en effet surprendre car, quand on s'interroge sur la fabrication du droit $^{3}$, on ne pense pas spontanément au juge. Pourtant, il est omniprésent, même inconsciemment, car il est amené, d'une façon ou d'une autre, à un moment ou un autre, à rappeler au législateur les limites de son action. Le premier réflexe qui consiste à se cacher derrière le principe de séparation des pouvoirs n'est donc pas pertinent et encore moins s'agissant de l'Union européenne ${ }^{4}$.

Aux termes de l'article 19 du Traité sur l'Union européenne, la Cour de justice de l'Union européenne (CJUE) se voit attribuer un rôle classique : on attend qu'elle « assure le respect du droit dans l'interprétation et l'application des traités ». Par ce biais, elle a ainsi largement participé à l'originalité du système juridique de l'Union. Robert Lecourt, Pierre Pescatore, plus récemment Antonio Tizzano et bien d'autres l'ont montré5. En outre, de nombreuses études ont été consacrées à la Cour de justice en tant qu'acteur de la gouvernance ${ }^{6}$, d'autres se sont aussi interrogés sur le gouvernement par la jurisprudence comme Loïc Azoulai ${ }^{7}$. Par conséquent, le positionnement de l'organe juridictionnel dans la gouvernance européenne ne constitue plus un sujet novateur pour lequel nos recherches auraient un quelconque intérêt.

Nos propos s'inscrivent plutôt dans une démarche pragmatique et se limiteront à savoir pourquoi et comment la question de la contribution du juge au « Mieux légiférer » est renouvelée par le paquet de $2015^{8}$. S'inscrivant dans la lignée de ses prédécesseurs ${ }^{9}$, les objectifs de cette nouvelle version sont bien connus : améliorer la réglementation pour obtenir de meilleurs résultats, garantir l'ouverture et la transparence, mettre en place des outils adéquats pour assurer l'efficacité et rafraîchir le corpus

2 Améliorer la réglementation pour obtenir de meilleurs résultats - Un enjeu prioritaire pour l’UE, COM (2015) 215 du 19 mai 2015 ; Accord interinstitutionnel entre le Parlement européen, le Conseil de l'Union européenne et la Commission européenne «Mieux légiférer » du 13 avril 2016 , JOUE L $123 \mathrm{du}$ 12.05.2016, p. 1 ; Améliorer la réglementation : de meilleurs résultats pour une Union plus forte, COM (2016) 615 du 14 septembre 2016 ; Le droit de l'Union européenne : une meilleure application pour de meilleurs résultats, fOUE C 18 du 19.01.2017, p. 10.

3 Cela dit, deux ouvrages majeurs mettent le juge au centre de leur questionnement sur la fabrication ou la fabrique du droit : B. LATOUR, La fabrique du droit - Une éthnographie du Conseil d'État, La découverte poche, 2004, 320 p. et P. Mвongo et A. VAuchez (éd.), Dans la fabrique du droit européen - Scènes, acteurs et publics de la Cour de justice des Communautés européennes, Bruylant, 2009,258 p.

4 V. notamment, T. GEORgopoulos, « Doctrine de séparation des pouvoirs et intégration européenne », in M. BLANQuET (éd.), La prise de décision dans le système de l'Union européenne, Bruylant, 2011, p. 3-30.

$5 \quad$ R. Lecourt, L'Europe des juges, Bruylant, 2007, 321 p.; P. Pescatore, Études de droit communautaire 1962-2007, Bruylant, 2008, 1006 p. ; Dans les mélanges dédiés à Paolo Mengozzi, l'Avocat général A. TizzAno montre l'influence de la Cour sur la construction du système juridique, son action au sein des États membres et les fondements et effets de sa jurisprudence relative aux droits fondamentaux in De Rome à Lisbonne : les juridictions de l'Union européenne à la croisée des chemins, Bruylant, 2013, 635 p.

V. notamment les travaux en science politique : S. SAURUGGER et F. TERPAN, « La Cour de justice au cour de la gouvernance européenne », Pouvoirs, 2014, p. 59 ; O. CostA, «La Cour de justice et le contrôle démocratique de l'Union européenne », Revue française de science politique, 2001, p. 881 ; A. VAUCHEZ, « La justice comme « institution politique » : retour sur un objet (longtemps) perdu de la science politique, Droit et société, 2006, p. 491 ; A. VAuchez, «A quoi « tient » la Cour de justice des Communautés européennes ?, Stratégies commémoratives et esprit de corps transnational », Revue française de science politique, 2010, p. 247.

7 Selon L. Azoulai, la Cour participe essentiellement au gouvernement de l'Union par l'élaboration de deux sortes d'opérateurs qui agissent sur la manière d'aborder les problèmes politiques et sociaux : les connecteurs qui servent à placer le problème de droit posé dans le contexte élargi d'exigences et les correcteurs qui encadrent le comportement des autorités politiques, administratives et judiciaires des États membres. In P. Mbongo et A. VAuchez (éd.), Dans la fabrique du droit européen - Scènes, acteurs et publics de la Cour de justice des Communautés européennes, op. cit. p. 168.

8 E. CARTIER avait déjà étudié la place de la justice dans la gouvernance du « Mieux légiférer » » en mettant en exergue les deux paramètres de l'équation -d'une part, bien juger pour mieux légiférer et d'autre part, bien légiférer et mieux juger- en les envisageant essentiellement sous le prisme de la jurisprudence nationale et de celle de la Cour européenne des droits de l'homme, in F. PÉRALDI-LENEUF et S. DE LA RosA (éd.), L'Union européenne et l'idéal de la meilleure législation, Pedone, 2013, p. 225.

F. PÉraldi-Leneuf et S. DE la Rosa (éd.), L’Union européenne et l'idéal de la meilleure législation, Pedone, 2013,246 p. ; E. VAN Den AbEELE, «L'agenda Mieux légiférer de l'Union européenne », Courrier hebdomadaire du CRISP, 2009, p. 5-79; D. MockLE, « La réglementation intelligente : règlementer mieux ou réglementer moins ? », RFDA, 2015, p. 1225 ; A. AlEmANno, « Dans quelle mesure le « Mieux légiférer » est-il meilleur? », Revue du droit de l'Union européenne, 2015, p. 509. 
législatif existant. Tous les acteurs se sont engagés à se mobiliser : la Commission bien évidemment, mais également le Parlement européen et le Conseil via l'Accord interinstitutionnel du 13 avril 2016 et l'adoption, en vertu de son article 7, d'une déclaration commune, relative à la programmation interinstitutionnelle annuelle signée par les présidents des trois institutions, qui expose les objectifs généraux et les priorités pour l'année ainsi que les dossiers d'importance politique majeure ${ }^{10}$.

Le but, affiché d'ailleurs dans la communication du 19 mai 2015, est de travailler de manière plus transparente et plus inclusive pour aboutir à des propositions de meilleure qualité et de faire en sorte que les règles existantes permettent de réaliser de manière plus efficace les grands objectifs sociétaux.

La place du juge est a priori très modeste, très peu de développements le concernant directement ou indirectement, ce qui renvoie finalement à une conception classique du rôle du juge mêlée à une méfiance de la procéduralisation.

Ainsi, la Communication du 19 mai 2015 rappelle un certain nombre d'exigences tenant notamment à l'interprétation et l'application du droit de l'UE qui font, du reste, largement écho aux paquets antérieurs et qui ont amené la Cour à développer une jurisprudence significative relative aux impératifs de qualité et d'efficacité de la norme comme l'a montré Rostane Mehdi ${ }^{11}$. À cet égard, on relèvera trois extraits pertinents dans l'accord interinstitutionnel : « Les trois institutions conviennent que le choix de la base juridique est une appréciation juridique qui doit se fonder sur des motifs objectifs susceptibles de contrôle juridictionnel » (pt 25 § 4); « La Commission continue de jouer pleinement son rôle institutionnel afin de veiller au respect des traités et de la jurisprudence de la Cour de justice de l’Union européenne » (pt 25 § 5) ; «Les trois institutions reconnaissent qu'il importe de veiller à ce que chaque institution puisse exercer ses droits et remplir ses obligations dans le cadre de la négociation et de la conclusion d'accords internationaux, tels qu'ils sont consacrés dans les traités et interprétés par la Cour de justice de l'Union européenne. Les trois institutions s'engagent à se réunir dans un délai de six mois à compter de l'entrée en vigueur du présent accord pour négocier des modalités pratiques plus efficaces en matière de coopération et d'échange d'informations dans le cadre des traités, tels qu'ils sont interprétés par la Cour de justice de l'Union européenne » (pt 40).

Par conséquent, le paquet 2015 envisage essentiellement la garantie que le contrôle du juge peut ainsi apporter à l'équilibre des pouvoirs.

En outre, on relève que les risques liés à la procéduralisation n'apparaissent plus aussi clairement que dans la Communication du 11 décembre $2002^{12}:$ « La Commission demeure cependant convaincue qu'une approche juridiquement contraignante de la consultation est à éviter pour deux

10 Déclaration commune sur les priorités législatives de l'Union européenne pour l'année 2017 signée le 13 décembre 2016.

11 R. MeHdi, «L'efficacité de la norme en droit de l'Union européenne », in M. FATIN-Rouge StéFAnini et autres (éd.), L'efficacité de la norme juridique, nouveau vecteur de légitimité ?, Bruylant, 2012, p. 295

12 Vers une culture renforcée de la consultation et du dialogue - Principes généraux et normes minimales applicables aux consultations engagées par la Commission avec les parties intéressées, COM (2002) 704. 
raisons. Premièrement, la frontière doit être claire entre les consultations engagées à l'initiative de la Commission avant l'adoption d'une proposition et le processus décisionnel formel et obligatoire qui y succède conformément aux traités. Deuxièmement, il faut éviter que les propositions de la Commission puissent être attaquées devant le juge communautaire pour défaut de consultation des parties intéressées. Cette approche trop légaliste serait incompatible avec la nécessité de mener les politiques en temps opportun, ainsi qu'avec les attentes des citoyens qui souhaitent que les institutions européennes concentrent davantage leurs efforts sur le contenu, et non sur les procédures ».

Dès lors, la place de la Cour de justice dans le contexte du « Mieux légiférer » est loin d'être principale dans les textes, mais elle demeure essentielle en pratique. Encore faudrait-il s'atteler à définir d'éventuels critères permettant d'évaluer l'impact juridictionnel des ambitions proclamées depuis 2015, on vise ici l'impact sur la juridiction bien sûr mais aussi l'impact de la juridiction.

Cette étude se veut nécessairement transversale et revendique sa superficialité, tant le sujet est large et fuyant, et tout le défi est d'offrir au lecteur une présentation qui ne soit ni la synthèse des autres brillantes études, ni redondante et encore moins hors sujet... Nous nous interrogerons donc sur la place et le rôle du juge dans cette mécanique et l'influence qu'il peut exercer ou qu'il peut subir. Aussi, nous analyserons ses capacités d'impulsion et d'adaptation dans cette quête de qualité, de rationalisation et au final de légitimité du droit de l'Union ${ }^{13}$. En définitive, il s'agit de réfléchir plus largement à l'office du juge et à son évolution dans le contexte du paquet « Mieux légiférer $»^{14}$. Nous inscrirons dès lors cette étude de la contribution de la CJUE aux exigences du « Mieux légiférer » dans une double approche, d'abord matérielle (1) et ensuite formelle voire inter-institutionnelle (2).

\section{1) Approche matérielle de la contribution de la CJUE au « Mieux légiférer»}

L'idée ici est de démontrer que, par ses arrêts, la Cour de justice peut donner un écho aux exigences du « Mieux légiférer » et contribuer ainsi à en assurer l'effectivité. La recherche a abouti à deux conclusions principales : la première est que la référence explicite aux instruments $\mathrm{du}$ « Mieux légiférer » est très exceptionnelle (1.1), la seconde est que la jurisprudence récente s'inscrit de façon plus claire dans le mouvement de légitimation et de d'effectivité du droit de l’Union (1.2).

\subsection{Une référence exceptionnelle aux instruments du « Mieux légiférer»}

Le premier réflexe du chercheur est de déterminer les occurrences des termes « Mieux légiférer », «gouvernance », « réglementation affûtée », « refit »... dans la jurisprudence de la Cour. On conviendra

\footnotetext{
13 Voir notamment F. Hervouet, « Brèves réflexions à propos de la légitimité de l’Union européenne », in B. BERTRAND (éd.), L’identité du droit de l'Union européenne, Mélanges en l'honneur de C. BLumann, Bruylant, 2015, p. 375-387.

14 V. pour une étude très générale les actes du colloque organisé par le Sénat les 29-30 septembre 2006, L’office du juge, 544 p. V. également les contributions et propositions contenues dans l'Étude annuelle 2016 du Conseil d'État, Simplification et qualité du droit, La documentation Française, $256 \mathrm{p}$.
} 
aisément que la démarche est on ne peut plus réductrice voire d'une systématicité déconcertante, mais elle a le mérite de confirmer que la Cour de justice n'envisage pas de front le respect des exigences tenant au « Mieux légiférer».

Les contentieux en matière fiscale fournissent une première illustration : le principe du « Mieux légiférer » est récurrent, mais la Cour ne fait que rappeler un considérant de la sixième directive, sans d'ailleurs en tenir compte par la suite ${ }^{15}$. La référence expresse au « Mieux légiférer » n'est donc ici pas particulièrement exploitable.

Une deuxième série d'exemples de références explicites se trouve dans les conclusions des avocats généraux.

Dans l'affaire du contrôle prudentiel des établissements de crédits ${ }^{16}$, l'avocat général Niilo Jääskinen n'a pas été convaincu par les arguments soulevés par le Royaume-Uni tenant au non-respect des principes de subsidiarité et de proportionnalité allant à l'encontre « des indications » données dans l'accord interinstitutionnel « Mieux légiférer ». Il conclut à cet égard que le législateur de l'Union européenne a dûment respecté toutes les exigences procédurales dans l'évaluation de la conformité de la proposition à ces principes et qu'il possède un large pouvoir d'appréciation en la matière.

Dans l'affaire des tabacs ${ }^{17}$, l'avocat général Juliane Kokott évoque avec ironie le décalage entre les ambitions et la pratique. Ainsi, après avoir rappelé la jurisprudence sur la motivation des actes, elle constate que le considérant 60 de la Directive en cause «se borne à constater lapidairement » que « les objectifs de la présente directive, à savoir le rapprochement des dispositions législatives, réglementaires et administratives des États membres en matière de fabrication, de présentation et de vente du tabac et des produits connexes, ne peuvent pas être atteints de manière suffisante par les États membres, mais peuvent, en raison de leurs dimensions et effets, l'être mieux au niveau de l'Union », pour en conclure que « celle-ci peut prendre des mesures, conformément au principe de subsidiarité consacré à l'article 5 [TUE] » (§ 289). Elle remarque alors : « On ne saurait soutenir que cette formulation, cantonnée en définitive à une reproduction modulaire du libellé même des dispositions pertinentes du traité UE, soit vraiment un brillant spécimen de la technique si célébrée $\mathrm{du}$ « mieux légiférer », que les institutions de l'Union se sont fixées comme objectif depuis quelque temps » (§ 290). «Certes, la présence d'une telle formule standard dans le préambule d'un acte de l'Union ne devrait pas à elle seule conduire à des conclusions hâtives sur le respect de l'obligation de motivation. Une telle formule invite néanmoins à constater la carence de la motivation de l'acte en cause. Si l'on peut déduire après tout de cette motivation que le législateur était lui-même convaincu de respecter le principe de subsidiarité, elle ne fait cependant pas clairement apparaître les réflexions

\footnotetext{
15 «Pour assurer que les dispositions sont présentées d'une façon claire et rationnelle, compatible avec le principe de mieux légiférer, il est opportun de procéder à la refonte de la structure et du libellé de la directive bien que cela ne doive, en principe, pas provoquer des changements de fond dans la législation existante. Un petit nombre d'amendements substantiels est néanmoins inhérent à l'exercice de refonte et devrait, en tout état de cause, être apporté. Les cas où ces amendements sont effectués sont repris de manière exhaustive dans les dispositions sur la transposition et l'entrée en vigueur de la directive. »

16 Conclusions rendues le 20 novembre 2014 dans l'affaire Royaume-Uni c./ Parlement européen et Conseil, aff. C-507/13.

17 V. notamment les conclusions rendues le 23 décembre 2015, Philip Morris Brands, aff. C-547/14.
} 
qu'il a exactement menées à propos de la problématique de la subsidiarité ni la profondeur de son analyse de cette question » (§ 291). Mais ce subtil rappel à l'ordre ne la conduit pas à conclure en faveur d'une déclaration d'invalidité. Elle retient en effet : « Il importe de noter en l'espèce que le législateur de l'Union a pu se fonder à la fois sur la motivation de la proposition de directive présentée par la Commission et sur une analyse exhaustive élaborée par ses services dans le cadre de l'analyse d'impact de la Directive. Les inconvénients résultant de réglementations nationales divergentes ainsi que les avantages d'une action à l'échelon de l'Union font l'objet d'un examen approfondi non seulement dans les développements consacrés spécifiquement au principe de subsidiarité, mais également dans de nombreuses autres sections de ces deux textes (§ 295). Par conséquent, «Il est ainsi établi à suffisance de droit que les institutions législatives de l'Union ont disposé d'une documentation complète à même d'étayer l'appréciation qu'elles ont portée sur le respect du principe de subsidiarité » (§ 296). Bref, aucune violation matérielle ou formelle n'est retenue, mais l'avocat général en profite pour ajouter : « Il convient toutefois de recommander vivement au législateur de l'Union de ne plus recourir à l'avenir à des formules aussi creuses que celle du considérant 60 de la Directive et d'étayer au contraire l'exposé des motifs de ses actes de développements sur le principe de subsidiarité suffisamment circonstanciés et plus étroitement ciblés sur chacune des mesures considérées » (§ 301). Dans ces arrêts ${ }^{18}$, la Cour ne sera pas aussi explicite et sévère, elle se contente d'affirmer : «En l'occurrence, il est constant que la proposition de directive présentée par la Commission ainsi que l'analyse d'impact établie par celle-ci comportent suffisamment d'éléments faisant apparaître d'une façon claire et non équivoque les avantages liés à une action entreprise au niveau de l'Union, plutôt qu'au niveau des États membres » (§ 226). «Dans ces conditions, il est établi à suffisance de droit que ces éléments ont permis tant au législateur de l'Union qu'aux parlements nationaux d'apprécier si ladite proposition était conforme au principe de subsidiarité, tout en étant de nature à permettre aux particuliers de prendre connaissance des motifs se rapportant à ce principe et à la Cour d'exercer son contrôle » (§ 227).

Enfin un ultime exemple de mention expresse, tiré d'un arrêt rendu par le Tribunal concernant les dispositifs médicaux ${ }^{19}$, nous renseigne sur le principal obstacle : la question de l'invocabilité des instruments juridiques du « Mieux légiférer ». Dans cette affaire, qui a pour objet un recours en indemnité visant à obtenir réparation du préjudice qu'il aurait prétendument subi à la suite de la violation par la Commission des obligations qui lui incombent en vertu de l'article 8 de la directive 93/42/CEE du Conseil, du 14 juin 1993, relative aux dispositifs médicaux, le requérant fait valoir que, en l'espèce, l'obligation, pour la Commission, d'adopter une décision découle du principe de bonne gouvernance, tel que décrit, d'une part, par le livre blanc sur la gouvernance européenne de 2001, et, d'autre part, par le Guide relatif à la mise en application des directives élaborées sur la base des dispositions de la nouvelle approche et de l'approche globale de la Commission. Le Tribunal répond : «Or, à cet égard, premièrement, il importe de rappeler que le livre blanc n'est qu'un document

18 CJUE, 4 mai 2016, Pologne c./ Parlement et Conseil, aff. 358/14 ; CJUE, 4 mai 2016, Pillbox 38, aff. C-477/14 ; CJUE, 4 mai 2016 , Philip Morris Brands, aff. C-547/14.

19 Trib., 21 janvier 2014, Christoph Klein, aff. T-309/10. 
contenant des propositions d'action de l'Union dans un domaine spécifique. À titre général, un livre blanc s'inscrit souvent dans le prolongement d'un livre vert, dont le but est de lancer un processus de consultation au niveau européen. Il s'agit, dès lors, d'une communication destinée à lancer un débat politique et non à créer des obligations à la charge de la Commission dans un cas comme celui de l'espèce. Deuxièmement, le guide, outre qu'il ne peut pas constituer le fondement juridique d'une obligation à charge de la Commission dans le sens allégué par le requérant, indique que les actions à entreprendre par les autorités nationales et par la Commission, dans le cadre de la gestion d'une violation des dispositions des directives dites « nouvelle approche », comme la directive 93/42, doivent être décidées, au cas par cas, en fonction du type de non-conformité constatée. En particulier, selon le guide, les cas où, comme celui de l'espèce, l'obligation de tenir à la disposition des autorités compétentes les informations requises par la directive, telles que la documentation technique ou les données cliniques, n'est pas entièrement remplie, constituent des illustrations de «non-conformité non substantielle » (§ 84 et 85 ). Dans ces cas, les autorités nationales sont tenues d'enjoindre au fabricant de mettre un terme à l'infraction, de restreindre ou d'interdire la mise sur le marché du produit concerné et, au besoin, d'assurer que ledit produit est également retiré du marché ». Il conclut qu' « aussi regrettable que fût l'absence de réaction de la Commission à la suite de la notification par les autorités allemandes de la décision d'interdiction du dispositif Inhaler, aucun comportement illégal de cette institution ne peut être déduit du fait qu'elle n'a pas adopté » la décision attendue.

\subsection{Une jurisprudence en phase avec les exigences du « Mieux légiférer »}

Indépendamment des mentions expresses qui restent donc exceptionnelles, le juge donne écho aux exigences énumérées dans le paquet « Mieux légiférer ». Ces exigences restent malgré tout classiques par rapport aux textes précédents, presque redondantes. On retiendra essentiellement une série de mots-clefs liés à l'adéquation des résultats aux objectifs poursuivis : l'Accord interinstitutionnel en livre une énumération qui nous permet d'établir une grille d'analyse des arrêts rendus depuis 2015 : analyse d'impact, consultation du public, évaluation, instruments législatifs, actes délégués et actes d'exécution, transparence et conditions du processus législatif, mise en œuvre et application de la législation de l'UE, simplification (refonte, harmonisation, reconnaissance mutuelle). Une recherche systématique des occurrences de ces termes aboutit à l'établissement d'une liste d'arrêts, qui pris séparément ne permettent certes pas de dévoiler le sens de la jurisprudence actuelle $e^{20}$, mais, qui, envisagés sous l'angle de « séries », laissent entrevoir une tendance qui nous aide à décrypter la contribution du juge au « Mieux légiférer » et, de façon réciproque, l’influence du « Mieux légiférer » sur le contrôle juridictionnel. Au fond, le juge, sollicité par l'ensemble des acteurs - institutions, États,

20 L'idée de « sens » est intéressante car elle englobe à la fois l'idée de direction et d'évolution, donc une démarche forcément dynamique, et renvoie également à un aspect plus statique tenant à la signification. Aussi serait-il opportun de s'interroger sur l'existence d'une démarche, d'une approche juridictionnelle spécifique. Le sens du paquet Mieux légiférer semble être celui d'une démarche résolument globale et générale embrassant l'amélioration de la législation tant sur la forme que sur le fond (V. sur ce point les contributions de F. PÉRALDI-LENEUF et de S. DE LA RosA, infra). 
personnes morales et physiques - contribue à garantir une sorte d'idéal démocratique inhérent à la recherche d'une norme de qualitée ${ }^{11}$. Il s'agit ici de dépasser, sans l'occulter, la problématique de la clarté et de la prévisibilité de la norme qui a déjà donné lieu à une jurisprudence ancrée au principe de sécurité juridique ${ }^{22}$. Pourtant, on peut s'interroger sur l'émergence de «blocs de jurisprudence » dans le contexte $\mathrm{du}$ 《 Mieux légiférer ${ }^{23}$ dans deux domaines principaux : celui de l'ouverture et de la transparence d'une part, et celui du respect de la répartition des pouvoirs d'autre part.

\section{La garantie de l'ouverture et de la transparence}

Une première série d'arrêts rend compte du souci d'accroître le champ de l'ouverture et de la transparence et d'en conforter également le respect.

D'une part, le contentieux relatif à l'Initiative citoyenne européenne (ICE), conçue comme un « instrument fondamental de la démocratie participative » appelle-t-elle la Commission à un effort particulier de «pédagogie ${ }^{24}$. Dans l'affaire $E f l e r^{25}$, le Tribunal a fait prévaloir une acception large de la notion d'acte juridique au sens de l'article $11 \S 4$ du TFUE, il n'a pas entendu limiter cette condition aux seuls actes juridiques de l'Union définitifs et produisant des effets juridiques à l'égard des tiers. Il se fonde sur le principe de démocratie, qui figure parmi les valeurs fondamentales sur lesquelles l'Union repose, de même que l'objectif spécifiquement poursuivi par le mécanisme de l'ICE, consistant à améliorer le fonctionnement démocratique de l'Union en conférant à tout citoyen un droit général de participer à la vie démocratique. En effet, il considère que la thèse défendue par la Commission, selon laquelle le Conseil et celle-ci jouiraient d'une légitimité démocratique indirecte suffisante pour adopter les actes juridiques qui ne déploient pas d'effets juridiques à l'égard des tiers, « aurait pour conséquence de limiter considérablement le recours au mécanisme de l'ICE comme instrument de participation des citoyens de l'Union à l'activité normative de celle-ci par le biais de la conclusion d'accords internationaux ». Par ailleurs, le Tribunal écarte l'argument de la Commission selon lequel les actes que la proposition d'ICE l'invite à soumettre au Conseil aboutiraient à une immixtion inadmissible dans le déroulement d'une procédure législative en cours : «En effet, le but poursuivi par l'ICE

21 Il est intéressant de noter en outre l'apport de l'arrêt du 27 octobre 2016, fames Elliott, aff. C-613/14 dans lequel la Cour rappelle que la nature non contraignante d'un acte de droit de l'Union, comme c'est le cas concernant les normes techniques concernées, ne la prive pas de sa compétence d'interprétation, et que c'est la publication des normes techniques au fournal officiel de l'Union européenne qui confère aux produits qui satisfont aux spécifications de la norme une présomption de conformité aux exigences de la directive. Ainsi, la norme en cause fait bien partie du droit de l'Union, alors même que son élaboration est confiée à un organisme de droit privé, ce qui suffit pour conclure à une compétence d'interprétation de la Cour.

22 Pour une confirmation récente s'agissant des fonds structurels, V. Trib., 20 octobre 2016, République Tchèque c./ Commission, aff. T-141/15, $\S 72$ : «Ce principe exige que le droit de l'Union soit clair et son application prévisible pour ceux qui sont concernés. Cet impératif requiert que tout acte visant à créer des effets juridiques emprunte sa force obligatoire à une disposition du droit de l'Union qui doit expressément être indiquée comme base juridique et qui prescrit la forme juridique dont l'acte doit être revêtu (voir arrêt du 19 juin 2015, Italie/Commission, T-358/11, EU:T:2015:394, point 123 et jurisprudence citée) ».

23 V. B. BERTRAND, «Les blocs de jurisprudence », RTDE, 2012, pp. 741 et s. qui sont présentés comme un « outil conceptuel » transformant une « jurisprudence inexprimée en une jurisprudence intelligible ».

24 V. Conclusions P. Mengozzi dans l'affaire Anagnostakis, 7 mars 2017, C-589/15 (pourvoi) : «Il s'agit d'un instrument fondamental pour la démocratie participative européenne qui peut se révéler délicat à mettre en œuvre. En effet, d'une part, si l'idée est celle de donner aux citoyens un rôle actif dans la construction du droit de l'Union, il faut veiller à ne pas enserrer ce droit d'initiative dans des conditions, de forme ou de fond, trop strictes ou trop complexes - et donc, au final, peu compréhensibles - à satisfaire pour le non-spécialiste du droit de l'Union ; mais, d'autre part, l'on ne saurait ignorer que l'ordre juridique de l'Union est régi par le principe d'attribution des compétences, et la démocratie participative, à laquelle l'article 11, paragraphe 4, TUE tente de donner vie, ne peut donc s'exercer que dans ces limites ».

25 Trib., 10 mai 2017, Efler, aff. T-754/14. 
est de permettre aux citoyens de l'Union de participer davantage à la vie démocratique de l'Union, notamment, en exposant dans le détail à la Commission les questions soulevées par l'ICE, en invitant cette institution à soumettre une proposition d'acte juridique de l'Union après avoir, le cas échéant, présenté l'ICE lors d'une audition publique organisée au Parlement, conformément à l'article $11 \mathrm{du}$ règlement $n^{\circ}$ 211/2011, partant, en suscitant un débat démocratique sans avoir à attendre l'adoption de l'acte juridique dont la modification ou l'abandon est en définitive souhaité ». Par conséquent, « loin de représenter une immixtion dans le déroulement d'une procédure législative en cours, la proposition d'ICE constitue une expression de la participation effective des citoyens de l'Union à la vie démocratique de celle-ci, sans remettre en cause l'équilibre institutionnel voulu par les traités ».

L'importance reconnue par le juge à l'Initiative citoyenne européenne implique en outre un contrôle strict de l'obligation de motivation commel'a rappelé le Tribunal dans les arrêts Anagnostakis ${ }^{26}$ et One million signatures for diversity in Europe ${ }^{27}$.

D'autre part, la jurisprudence actuelle va dans le sens d'une interprétation large du principe d'accès aux documents que ce soit dans le cadre d'affaires en matière de protection de l'environnement ${ }^{28}$, celles ayant donné lieu à un recours en manquement ${ }^{29}$ ou dans le cadre de mécanismes pré-contentieux ${ }^{30}$.

Ce souci d'ouverture et de transparence est également exprimé par les États dans le processus législatif comme en témoigne par exemple le recours introduit par la Pologne le 10 mars 2017 relatif à la directive sur la réduction des émissions nationales de certains polluants atmosphériques. La République de Pologne souligne que n'ont pas été mis à disposition ou publiés les principes fondamentaux sur la base desquels sont définis les engagements nationaux de réduction des émissions pour 2030 et les années suivantes. Il n'y a pas d'informations concernant les hypothèses relatives à la structure technologique de secteurs particuliers sur lesquelles sont fondées les projections relatives aux émissions en 2030. L'absence d'informations a empêché la vérification de la solidité des projections d'émissions telles qu'établies pour l'année 2030. "Deuxièmement, on ne sait pas sur la base de quelle formule a été calculé l'objectif général « santé » concernant la réduction de la mortalité dans l'Union en vue des engagements de réduction des émissions pour l'ensemble de l'Union et les États membres ». Par conséquent, « le raisonnement des institutions qui ont adopté la directive n'a pas été présenté de manière claire et univoque en ce qui concerne les engagements de réduction précités ». Par ailleurs, la République de Pologne souligne que, compte tenu de l'importante incidence attendue des engagements de réduction pour 2030 et les années suivantes sur l'économie et la société dans les États membres, l'évaluation des effets réalisée par la Commission est insuffisante.

26 Trib., 30 septembre 2015, Anagnostakis c. Commission, aff. T-450/12 : « L'article 4, paragraphe 3, second alinéa, du règlement n² 211/2011, selon lequel la Commission informe les organisateurs des motifs du refus d'enregistrement d'une proposition d'ICE, constitue l'expression spécifique de ladite obligation de motivation dans le domaine de l'ICE ».

27 Trib., 3 février 2017, One million signatures for diversity in Europe, aff. T-646/13.

28 Concernant des documents détenus par la Commission établissant le respect de la législation par les États : CJUE, 16 juillet 2015 , ClientEarth, aff. C-612/13 : la présomption générale de confidentialité ne s'applique pas aux documents qui, au moment de la décision refusant l'accès, n'ont pas été versés dans un dossier relatif à une procédure administrative ou juridictionnelle en cours. De façon plus générale, V. la contribution d'E. BRossET.

29 CJUE, 18 juillet, Breyer, aff. C-213/15.

30 CJUE, 11 mai 2017, Suède c./ Commission, aff. C-562/14. 
Cela nous permet de rappeler l'intérêt des analyses d'impact auxquelles le juge n'hésite pas à se référer notamment pour justifier l'intervention de l'UE. Par exemple, dans l'une des affaires tabacs ${ }^{31}$, la Cour se fonde, pour établir l'existence de divergences entre les réglementations nationales, non seulement sur la directive mais renvoie aussi à l'analyse d'impact pour conclure que suffisamment d'éléments avaient été apportés au législateur faisant dès lors apparaître d'une façon claire et non équivoque les avantages liés à une action entreprise au niveau de l'Union, plutôt qu'au niveau des États membres. L'avocat général concluait d'ailleurs dans cette affaire que « les institutions législatives de l'Union ont disposé d'une documentation complète à même d'étayer l'appréciation qu'elles ont portée sur le respect du principe de subsidiarité ».

\section{La garantie de la répartition des pouvoirs}

La jurisprudence recensée montre comment les enjeux politiques sont saisis par le juge dans le cadre du contentieux de la base juridique et des compétences et pouvoirs des institutions. Les arrêts récents sont d'ailleurs révélateurs de la volonté de la Cour de donner au principe d'équilibre institutionnel une véritable substance ${ }^{32}$. Il faut dire que tant les institutions que les États sont particulièrement insistants à cet égard ${ }^{33}$. La présentation de cette seconde série d'arrêts s'opérera en passant en revue les institutions bénéficiaires de chacune des positions adoptées par le juge. Il est d'ailleurs notable que l'équilibre soit finalement assez harmonieux grâce à une interprétation des dispositions du Traité au regard des responsabilités respectives des institutions.

Tout d'abord, et de façon générale, le législateur est conforté dans sa compétence pour l'adoption de mesures impliquant un choix politique. Dans les affaires jointes ayant donné lieu à l'arrêt Parlement et Commission c./ Conseil du $1^{\mathrm{er}}$ décembre 2015, la Cour, statuant en grande chambre, a accueilli les recours introduits par le Parlement et la Commission visant à l'annulation du règlement n 1243/2012, modifiant le règlement $\mathrm{n}^{\circ} 1342 / 2008$ établissant un plan à long terme pour les stocks de cabillaud et les pêcheries exploitant ces stocks. La Cour a rappelé sa jurisprudence antérieure selon laquelle l'adoption de mesures qui impliquent un choix politique, en ce qu'elle suppose une évaluation de leur nécessité pour poursuivre les objectifs afférents aux politiques communes, doit être réservée au législateur de l'Union statuant sur le fondement de l'article $43 \S 2$ TFUE. En revanche, conformément à l'article $43 \S 3$ TFUE, l'adoption des mesures relatives à la fixation et à la répartition des possibilités de pêche ne nécessite pas une telle appréciation dès lors que de telles mesures ont un caractère principalement technique et qu'elles sont censées être prises pour l'exécution des dispositions adoptées sur la base de l'article 43 § 2 TFUE. Elle conclut que les modifications apportées par le règlement définissent le cadre juridique dans lequel les possibilités de pêche sont établies et attribuées ;

\footnotetext{
31 CJUE, 4 mai 2016, Pologne c./ Parlement européen et Conseil, aff. C-358/14.

32 B. BERTRAND note d'ailleurs que les arrêts de 2015 changent la donne : « Pour la première fois, l'équilibre institutionnel semble avoir une normativité accrue et une nature juridique précise : celle d'un principe », in « Le principe d'équilibre institutionnel : une double inconstance, Europe, juin 2016.

33 Par exemple, V. le recours introduit par la République slovaque le 2 décembre 2015 (C-643/15) tendant à l'annulation de la décision du Conseil instituant des mesures provisoires en matière de protection internationale au profit de l'Italie et de la Grèce et soulevant pas moins de six moyens dont quatre fondés sur le respect du principe d'équilibre institutionnel et trois sur le principe de démocratie représentative.
} 
elles résultent ainsi d'un choix politique ayant un impact à long terme sur le plan pluriannuel, de sorte qu'elles auraient donc dû être adoptées sur la base de l'article $43 \S 2$ TFUE.

Ensuite, dans les affaires liées au statut de Mayotte $^{34}$, la Cour consolide la compétence du Conseil pour adopter des mesures spécifiques en vertu de l'article 349 TFUE relatif aux régions ultrapériphériques, évinçant ainsi une intervention plus étendue en la matière des autres institutions. La Cour, ayant examiné les objectifs et le contenu de chacun des actes attaqués, a considéré que les mesures qui y figurent ont été arrêtées en tenant compte de la situation économique et sociale structurelle de Mayotte, au sens de l'article 349 TFUE et présentent un lien de rattachement avec des caractéristiques et contraintes particulières de cette région. Par conséquent, la Commission et le Parlement ne sont pas fondés à soutenir que les actes attaqués ne pouvaient avoir pour base juridique l'article 349 TFUE, ce qui conforte considérablement la position du Conseil en la matière.

De la même façon, la Cour confirme le Conseil dans sa fonction d'élaboration de l'action extérieure de l'Union dans l'arrêt Conseil c./ Commission ${ }^{35}$ du 28 juillet 2016 en annulant la décision de la Commission prévoyant, d'une part, d'approuver un addendum au mémorandum d'entente entre l'Union et la Confédération suisse concernant la participation financière suisse dans l'Union élargie et, d'autre part, d'autoriser certains membres de la Commission à signer cet addendum au nom de l'Union.

En outre, dans l'arrêt Parlement c./ Conseil du 16 avril 2015, la place du Parlement européen est sauvegardée ${ }^{36}$. En effet, la Cour a annulé les décisions du Conseil mettant sous contrôle dans toute l'Union les nouvelles substances psychoactives. Ces décisions avaient été adoptées sans consultation du Parlement. La Cour a tout d'abord rappelé que, dans la mesure où les règles relatives à la formation de la volonté des institutions de l'Union sont établies par les traités et ne sont à la disposition ni des États membres ni des institutions elles-mêmes, seuls les traités peuvent, dans des cas particuliers, habiliter une institution à modifier une procédure décisionnelle qu'ils établissent. Dès lors, reconnaître à une institution la possibilité d'établir des bases juridiques dérivées, que ce soit dans le sens d'un renforcement ou dans celui d'un allégement des modalités d'adoption d'un acte, reviendrait à lui attribuer un pouvoir législatif qui excède ce qui est prévu par les traités. Ce principe s'applique non seulement à l'adoption d'actes législatifs, mais également aux bases juridiques prévues dans un acte de droit dérivé qui permettent l'adoption de mesures d'exécution de cet acte en renforçant ou en allégeant les modalités d'adoption de telles mesures prévues par les traités.

Dans le même sens, la Cour, dans l'arrêt Parlement c./ Conseil du 22 septembre 2016 ${ }^{37}$, a accueilli le recours en annulation introduit par le Parlement contre les décisions d'exécution accordant à quatre États membres l'accès à un système d'échange d'informations concernant des profils ADN, des empreintes digitales et certaines données relatives à l'immatriculation des véhicules.

\footnotetext{
CJUE, 15 décembre 2015, Parlement et Commission c./ Conseil, aff. jtes C-132/14 à C-136/14

CJUE, 28 juillet 2016, Conseil c./ Commission, aff. C-660/13.

CJUE, 16 avril 2015, Parlement c./ Conseil, aff. jtes C-317/13 et C-679/13.

CJUE, 22 septembre 2016, Parlement c./ Conseil, aff. jtes C-14/15 et C-116/15.
} 
Allant même plus loin, la Cour, a annulé, dans l'arrêt Parlement c./ Conseil du 14 juin $2016^{38}$ la décision concernant la signature et la conclusion de l'accord entre l'Union européenne et la République unie de Tanzanie relatif aux conditions de transfert, de la force navale placée sous la direction de l'Union européenne à la République unie de Tanzanie, des personnes soupçonnées d'actes de piraterie et des biens associés saisis. Ainsi, l'examen de l'accord UE-Tanzanie ayant démontré qu'il relève de manière prépondérante de la $\mathrm{PESC}$, et non de la coopération judiciaire en matière pénale ou de la coopération policière, la Cour a constaté que la décision concernant sa signature et sa conclusion a correctement été adoptée suivant la procédure spécifique aux accords portant sur la PESC. Cela dit, tout en rappelant l'absence de participation du Parlement aux procédures de négociation et de conclusion des accords relevant du domaine de la PESC, la Cour a ensuite jugé que l'exigence d'information du Parlement en vertu de l'article 218 § 10 TFUE s'étend également aux résultats intermédiaires auxquels les négociations aboutissent. Ainsi, le Conseil aurait dû, en l'espèce, communiquer le texte du projet d'accord et celui du projet de décision ; la méconnaissance de cette exigence d'information ayant empêché le Parlement d'exercer son droit de regard en matière de PESC et, le cas échéant, de faire valoir son point de vue en ce qui concerne la base juridique correcte sur laquelle la décision concernant la signature et la conclusion de l'accord devait se fonder, la Cour a donc annulé la décision attaquée pour violation d'une forme substantielle.

Enfin, concernant la Commission, sa position est aussi confortée : positivement, dans la reconnaissance de son droit de retrait de proposition législative et négativement, dans le strict respect de la distinction entre actes délégués et actes d'exécution.

Dans l'arrêt Conseil c./ Commission du 14 avril 2015'39, la Cour a rejeté le recours en annulation introduit par le Conseil contre la décision de la Commission par laquelle celle-ci avait retiré sa proposition de règlement du Parlement et du Conseil établissant les dispositions générales relatives à l'assistance macrofinancière aux pays tiers. Elle considère qu'il résulte des dispositions combinées de l'article 17 $\S 2$ TUE ainsi que des articles 289 TFUE et 293 TFUE que le pouvoir de la Commission, dans le cadre de la procédure législative ordinaire, ne se résume pas à présenter une proposition et, par la suite, à favoriser les contacts et à chercher à rapprocher les positions du Parlement et du Conseil. De même qu'il revient, en principe, à la Commission de décider de présenter, ou non, une proposition législative et, le cas échéant, d'en déterminer l'objet, la finalité et le contenu, elle a, aussi longtemps que le Conseil n'a pas statué, le pouvoir de modifier sa proposition, voire, au besoin, de la retirer. Cette faculté de retirer une proposition ne saurait toutefois investir cette institution d'un droit de veto dans le déroulement du processus législatif, qui serait contraire aux principes d'attribution des compétences et de l'équilibre institutionnel. Dès lors, le pouvoir de retrait de la Commission est indissociable du droit d'initiative et reste tout à fait cohérent dans le contexte du « Mieux légiférer » et du toilettage qu'il induit.

De même, concernant le volet international, la Cour a rejeté le recours en annulation introduit par le Conseil contre la décision de la Commission de présenter un exposé écrit au nom de l’Union

38 CJUE, 14 juin 2016, Parlement c./ Conseil, aff. C-263/14.

39 CJUE, 14 avril 2015, Conseil c./ Commission, aff. C-409/13, V. également la contribution de S. DE LA RosA. 
au Tribunal international du droit de la mer (TIDM) ${ }^{40}$. Pour écarter l'argument de certains États membres selon lequel l'article 335 TFUE sur la représentation de l'Union par la Commission devant les juridictions nationales ne concernerait pas les procédures devant les juridictions internationales, la Cour a rappelé que cette disposition constitue l'expression d'un principe général en vertu duquel l'Union possède la capacité juridique et est représentée, à cet effet, par la Commission. Ainsi l'article 335 TFUE offre à cette institution un fondement pour représenter l'Union devant le TIDM.

La Cour a en revanche tempéré les velléités de la Commission qui souhaitait accroître ses pouvoirs en matière d'actes non législatifs, ce qui a donné l'occasion au juge d'enrichir sa jurisprudence sur les actes délégués et les actes d'exécution ${ }^{41}$.

Par l'arrêt Commission c./ Parlement et Conseil ${ }^{42}$ du 16 juillet 2015, la Cour a rejeté le recours introduit par la Commission et visant à l'annulation d'une disposition, qui lui conférait un pouvoir délégué, du règlement modifiant le règlement $n^{\circ}$ 539/2001 du Conseil fixant la liste des pays tiers dont les ressortissants sont soumis à l'obligation de visa pour franchir les frontières extérieures des États membres et la liste de ceux dont les ressortissants sont exemptés de cette obligation. La Cour apporte des précisions sur la distinction entre l'octroi d'un pouvoir délégué et l'octroi d'un pouvoir d'exécution. Ainsi, la Commission, en exerçant un pouvoir d'exécution, contrairement aux pouvoirs qu'elle détient dans le cadre de l'exercice d'un pouvoir délégué, ne peut modifier ni compléter l'acte législatif, même dans ses éléments non essentiels. Ensuite, la Cour a souligné que ni l'existence ni l'étendue du pouvoir d'appréciation conféré à la Commission par l'acte législatif ne sont pertinentes aux fins de déterminer si l'acte à adopter relève de l'article 290 TFUE ou de l'article 291 TFUE.

Dans l'arrêt Parlement c./ Commission du 17 mars 2016 ${ }^{43}$, la Cour a précisé l'étendue du pouvoir délégué à la Commission de compléter un acte législatif, au sens de l'article 290 § 1 TFUE. S'agissant du régime des délégations prévu à l'article 290 TFUE, la Cour a rappelé qu'un acte législatif peut déléguer à la Commission le pouvoir d'adopter des actes non législatifs de portée générale qui « complètent » ou « modifient » certains éléments non essentiels de l'acte législatif et que ces deux catégories de pouvoirs délégués se distinguent nettement. Lorsque la Commission exerce un pouvoir de compléter un acte législatif, son mandat est limité au développement en détail, dans le respect de l'intégralité de l'acte législatif arrêté par le législateur, des éléments non essentiels de la réglementation concernée que le législateur n'a pas définis. En revanche, la délégation d'un pouvoir de modifier un acte législatif vise à autoriser la Commission à amender ou à abroger des éléments non essentiels édictés dans cet acte par le législateur.

Au-delà de ces deux séries d'arrêts que nous avons regroupés sous les thématiques d'ouverture et de respect de la répartition des pouvoirs, il conviendra à l'avenir de porter une attention particulière à l'impact juridictionnel des priorités annoncées par la Commission dans la communication Une

\footnotetext{
CJUE, 6 octobre 2015, Conseil c./ Commission, aff. C-73/14.

V. plus précisément la contribution de L. GuILloud-ColliaT.

CJUE, 16 juillet 2015, Commission c./ Parlement et Conseil, aff. C-88/14
}

CJUE, 17 mars 2016, Parlement c./ Commission, aff. C-286/14. 
meilleure application pour de meilleurs résultats dans laquelle elle laisse entrevoir une évolution des recours en manquements qu'elle initie. Priorité sera donnée aux cas dans lesquels les États membres n'ont pas communiqué leurs mesures de transposition ou dans lesquels ces mesures ont mal transposé les directives, les cas dans lesquels les États membres ne se sont pas conformés à un arrêt de la Cour de justice ou encore les cas dans lesquels ils ont gravement porté atteinte aux intérêts financiers de l'UE ou enfreint les compétences exclusives de l'UE. De plus, la Commission engagera systématiquement une procédure d'infraction si des règles nationales ou des pratiques générales font obstacle au bon fonctionnement du renvoi préjudiciel à la Cour de Justice ou si le droit national empêche les juridictions nationales de donner la primauté au droit de l'UE. Elle engagera également une procédure d'infraction lorsque le droit national ne prévoit pas de procédures de recours efficaces en cas d'infraction au droit de l'UE ou empêche d'une autre manière les systèmes judiciaires nationaux de garantir l'application effective du droit de l'UE conformément aux exigences de l'état de droit et à l'article 47 de la charte des droits fondamentaux de l'UE.

Hasard des recours ou rendez-vous des volontés, il semble bien que la place du juge ne puisse pas être marginalisée dans la mise en œuvre du « Mieux légiférer ». Pourtant, l'approche scientifique par recherche d'occurrences et la systématisation conceptuelle par blocs de jurisprudence ne permettent pas de livrer toute la richesse de l'impact juridictionnel. Aussi convient-il de s'interroger sur des instruments -plus opérationnels- aptes à faire du juge un acteur à part entière du « Mieux légiférer».

\section{2) Approche formelle et inter-institutionnelle de la contribution de la CJUE au « Mieux Légiférer»}

L'approche plus formelle et inter-institutionnelle renvoie davantage à une étude tournée vers la structure, les procédures, la méthode et les instruments. Elle est évidemment complémentaire de l'approche matérielle qui en est à la fois le corollaire et la résultante.

Cette approche plus procédurale est d'ailleurs au cœur des préoccupations de la Cour de justice. Ainsi, dans un nouveau format réduit de son rapport annuel 2016 intitulé « Panorama de l'année », a été insérée une cinquième partie consacrée aux perspectives («Regards vers l'avenir ») : la qualité de la justice, « un défi permanent ». Trois principaux axes sont énumérés : la réforme de l'architecture juridictionnelle, la refonte des règles de procédure, la modernisation et l'actualisation des méthodes de travail ${ }^{44}$. En outre, une attention particulière est désormais portée à la « justice en réseau $»^{45}$.

\footnotetext{
44 K. Andova et E. Von BARdeleben, « La refonte du règlement de procédure du Tribunal de l'Union européenne : pour une efficacité juridictionnelle renforcée, Europe, juillet 2015 ; Entretien de J.-C. BоNichot « Comment mettre le système juridictionnel de l'Union en ordre de bataille pour les années à venir ? », Europe, juin 2015.

V. également concernant les juges nationaux : «Tableau de bord de la justice dans l'Union européenne » élaboré par la Commission (COM (2016) 199 final, 11 avr. 2016) : l'objectif de ce document est de fournir un aperçu comparatif du fonctionnement, mais surtout de la qualité, de l'efficacité et de l'indépendance des systèmes juridictionnels dans les différents États membres. Les principaux indicateurs pris en compte sont constitués par des données quantitatives. De nouveaux indicateurs ont été introduits notamment sur la qualité des normes, de la formation, des études et de l'aide juridictionnelle.

45 V. l'intervention du Vice-Président du Conseil d’État, Jean-Marc SAUvé, lors du forum des magistrats organisé à Luxembourg en mars 2017 : « La justice européenne en réseau, élément indispensable d'une justice de qualité » (site du Conseil d’État).
} 
$\mathrm{Au}$ fond, la problématique des outils dépasse l'aspect simplement organisationnel pour devenir un véritable enjeu global non seulement de la qualité de la justice, de la qualité de ses décisions, mais aussi de la qualité de la norme passée au crible juridictionnel. Comment les procédures en place contribuent-elles aux exigences du « Mieux légiférer »? Une première contribution résulte de l'office classique du juge : sa participation à l'application du droit de l'UE (2.1). Une seconde voie reste sans doute à explorer : celle de sa participation plus visible à l'élaboration du droit de l'UE (2.2).

\subsection{La contribution par l'application et l'interprétation du droit de l'Union européenne}

L'office du juge consiste avant tout à appliquer le droit de l'Union élaboré par le législateur et/ou le pouvoir exécutif. Le paquet « Mieux légiférer » de 2015 donne toute son importance à l'application de la législation, même si ce sont avant tout les États membres qui sont les principaux débiteurs en la matière ${ }^{46}$. La contribution du juge peut être mise en lumière grâce aux méthodes d'interprétation, grâce à la structure du système juridictionnel et enfin grâce aux outils de recherche jurisprudentielle.

Grâce à des méthodes éprouvées depuis déjà longtemps, l'interprétation du juge profite à l'efficacité du droit de l'UE en garantissant l'unité de l'ordre juridique européen et l'uniformité de son application $^{47}$. Le juge de l'Union assume donc le rôle politique induit par le processus d'intégration, mais on attend aussi de lui qu'il participe à la simplification de la règle et à l'amélioration de la qualité du droit, autrement dit on attend de lui qu'il clarifie ${ }^{48}$. De ce point de vue, les réflexions sur le style des arrêts de la Cour sont significatives de l'évolution de la normativité qu'il convient d'y attacher ${ }^{49}$.

Mais cette clarification est une œuvre collective dans le système juridictionnel de l'Union : Tribunal, Cour et juges nationaux ${ }^{50}$. Ces derniers ont un rôle tout à fait moteur à jouer dans la clarification du droit de l'Union notamment dans le cadre du renvoi préjudiciel et il est intéressant de voir que leur participation peut varier en analysant la motivation des questions renvoyées : parfois très neutres voire laconiques, ils peuvent, par une motivation plus fournie et précise, pousser la Cour dans ses derniers retranchements ${ }^{51}$. Par conséquent, grâce à la structure du système juridictionnel, l'entreprise de clarification de la règle est démultipliée, - en théorie en tout cas, nonobstant les arrêts parfois pour le moins obscurs de la Cour... Cette logique de réseau existe par la force du renvoi préjudiciel, mais au-delà du seul aspect procédural se pose la question des outils aptes à développer cette relation bilatérale vers des ramifications plus centrifuges, non seulement verticalement mais aussi horizontalement ${ }^{52}$.

\footnotetext{
46 Partie VII de l'Accord interinstitutionnel du 13 avril 2016 et communication de la Commission publié le 19 janvier 2017.

47 V. R. MendI, «L'efficacité de la norme en droit de l'Union européenne », op. cit.

48 V. Étude annuelle 2016 du Conseil d'État, Simplification et qualité du droit, p. 119 et s : affirmer le rôle du juge dans son office de simplificateur.

49 L. Coutron, «Style des arrêts de la Cour de justice et normativité de la jurisprudence communautaire », RTDE, 2009, p. 643 et s. ; C. Schonberger, « Mi attendu, mi-dissertation, le style des décisions de la Cour de justice de l'Union européenne », Revue droit et société, 2015, n 91, p. 505-519 ; J.-C. Bonichot, « Le style des arrêts de la Cour de justice », Fustice et Cassation, 2013, p. 253-261.

50 O. Dubos, «Que reste-t-il de l'autonomie procédurale du juge national ? Histoire de l'attribution d'une compétence au nom de l'effectivité et de l'efficacité du droit de l'Union », in E. Neframi (éd.), Renvoi préjudiciel et marge d'appréciation du juge national, Bruylant, 2015, p. 159-182. 51 L. Coutron, distingue le juge national « simple courroie de transmission », "force de questionnement » et « force de proposition », in « La motivation des questions préjudicielles », in E. NeFrAMI, op.cit., supra.

52 V. Intervention de J.-M. SAuvé, op. cit.
} 
Grâce aux outils d'échange, de communication, de diffusion, de valorisation, la contribution du juge $\mathrm{au}$ « Mieux légiférer » serait rendue plus visible. Cela renvoie au partage de l'information dont le citoyen et les entreprises, et bien sûr l'Union européenne, sont finalement les bénéficiaires. La dimension instrumentale est bien présente dans les textes du « Mieux légiférer » et notamment dans la Communication du 19 mai 2015 qui y consacre tout un chapitre «Agir de manière plus efficace : de meilleurs outils pour de meilleurs politiques ». Cela dit, nous nous concentrerons ici sur les outils servant à évaluer la contribution du juge européen à la mise en œuvre du « Mieux légiférer » et notamment ceux mis en place par la Cour via le site internet Curia. Nous reprendrons ici la présentation qu'en fait la Cour avec nos commentaires sur leur utilité dans la perspective du « Mieux légiférer ».

Le formulaire permet une recherche assez complète grâce à l'activation de nombreux critères : numéro, parties, document, dates, mots du textes, matière... La liste de résultats est souvent très longue et le maniement des critères requiert une certaine habileté. De la même façon, l'accès numérique à la jurisprudence permet de retrouver une information pour chacune des affaires introduites. Il faut bien évidemment connaître soit le numéro de l'affaire, soit la date, soit le nom des parties.

De façon plus thématique, le Répertoire de jurisprudence regroupe, de manière systématique, les sommaires des arrêts et des ordonnances de la Cour de justice, du Tribunal et du Tribunal de la fonction publique. Le plan de classement, subdivisé initialement en huit parties, pour la période 1954-2009 et en un plan en neuf parties pour la jurisprudence à partir de 2010 : ordre juridique, cadre institutionnel, contentieux, politique intérieure de l'UE, association des pays et territoires d'outre-mer, politique extérieure, dispositions générales et finales, adhésions et fonction publique. Les sommaires reprennent les points de droit essentiels énoncés dans la décision considérée. Sont associées à chaque sommaire : une chaîne de mots-clés qui traduit, le contenu du point étudié, une cote de classement correspondant au domaine concerné et la référence aux points de la décision. La rubrique «Ordre juridique » comporte des sous-thèmes : sources du droit de l'Union, valeurs et objectifs, compétences et obligations respectives de l'UE et des États membres, droits fondamentaux, droit de l'Union et droit national, droit de l'Union et droit international, interprétation du droit de l'Union, principes démocratiques, non-discrimination et citoyenneté, dispositions d'application générale. Ce répertoire à jour doit être exploité en naviguant de rubriques en rubriques et donne les premiers éléments d'une recherche qu'il convient ensuite d'affiner.

De même, la table alphabétique des matières pourrait s'avérer extrêmement utile : elle répertorie les questions juridiques abordées dans les décisions de la Cour, du Tribunal et du Tribunal de la fonction publique, ainsi que dans les conclusions des avocats généraux, classées selon un ordre alphabétique. Plus d'une centaine de rubriques sont répertoriées, parfois très larges « Droit communautaire » ou très pointues «Coton ». Mais cette table ne recense les affaires que jusqu'en 2009.

De manière systématique, le Recueil est la publication officielle de la jurisprudence des juridictions qui composent la Cour de justice de l'Union européenne. D’abord publié sous forme papier, il l'est désormais exclusivement sous forme numérique depuis 2012. La page d'accès au Recueil 
présente les documents groupés par année et par mois, et ensuite par affaire. Reste la question de la politique de publication. Les critères sont les suivants : depuis le 1er mai 2004, font ainsi l'objet d'une publication au Recueil : les arrêts de l'assemblée plénière et de la grande chambre ; les arrêts rendus, dans les affaires préjudicielles, par les chambres à cinq juges et les chambres à trois juges ; les arrêts rendus, dans les affaires autres que préjudicielles, par les chambres à cinq juges et les chambres à trois juges, statuant avec conclusions de l'avocat général ; et les avis rendus au titre de l'article 218 § 11 TFUE. Sauf décision contraire de la formation de jugement concernée, ne sont donc en principe plus publiés au Recueil : les arrêts rendus, dans les affaires autres que préjudicielles, par les chambres à trois ou à cinq juges statuant sans conclusions de l'avocat général, et les ordonnances. Depuis le mois de septembre 2011, les chambres à trois ou à cinq juges peuvent par ailleurs décider, à titre exceptionnel, de ne pas publier au Recueil un arrêt préjudiciel. Le Recueil général comporte également des éléments d'information sur ces décisions ${ }^{53}$.

De manière synthétique, le Rapport annuel, décliné aussi en format plus réduit et plus pédagogique depuis 2016 - «Panorama »- présente l'activité judiciaire aussi bien d'un point de vue statistique que d'un point de vue thématique : droits fondamentaux, citoyenneté, dispositions institutionnelles... Il s'agit essentiellement d'une présentation des résumés des arrêts les plus marquants dans chacun des domaines.

Les communiqués de presse permettent de jeter un coup de projecteurs sur certains arrêts ou certaines conclusions, sans pour autant que les critères de leur publication ne soient clairement définis.

Au fond, plus globalement, cela renvoie à la politique de communication de la Cour et la question de l'accès et de la compréhension du sens de ses décisions : autrement dit la question de la pédagogie reste entièrement posée ${ }^{54}$. Il est vrai que d'autres juridictions - internationales ou nationales - ont développé ces dernières années des publications à vocation informative plus accessibles ${ }^{55}$.

Il ressort de l'énumération et de l'utilisation de ces divers outils que la politique jurisprudentielle et/ou le sens des priorités de la Cour ne sont pas très visibles et que dès lors sa contribution au « Mieux légiférer » reste largement à l'appréciation doctrinale ${ }^{56}$.

53 Pour comparaison : le Recueil Lebon placé, sous le haut patronage du Conseil d'État, permet la publication des arrêts les plus importants, la partie Table présente les arrêts de moindre importance et des renvois à la jurisprudence antérieure permettent de connaître l'apport jurisprudentiel de la décision. Les analyses sont rédigées par les responsables du centre de recherches et de diffusion juridiques du Conseil d'État.

54 L. Coutron (éd.), Pédagogie judiciaire et application des droits communautaire et européen, Bruylant, 2012, 320 p.

55 V. notamment les efforts déployés par la Cour européenne des droits de l'homme : aperçu des affaires, lettres d'information sur la jurisprudence, guides sur la jurisprudence et rapports de recherche, manuels de droit européen (récemment Manuel de droit européen d'accès à la justice) fiches thématique et fiches par pays...De même, le Conseil d'État publie sur son site : analyses mensuelles de jurisprudence, dossiers thématiques, discours et interventions, études...

56 Les conclusions de l'Avocat Général M. ВовEK dans l'affaire Breyer (C-213/15) sont particulièrement intéressantes à cet égard : à l'occasion d'une affaire concernant le droit d'accès aux documents, il développe son argumentaire autour de la justice démocratique et de la qualité de la justice, V. pt 98 et s. des conclusions rendues le 21 décembre 2016 : « Ainsi, c'est notamment par le biais de l'ouverture que les juridictions deviennent plus responsables à l'égard des citoyens. L'ouverture des juridictions favorise leur caractère démocratique en permettant aux citoyens de surveiller l'exercice du pouvoir judiciaire, en garantissant que ces derniers y participeront par le débat public et, en définitive, en améliorant la compréhension des décisions de justice. L'ouverture dont font preuve les juridictions finit ainsi par alimenter leur légitimité via les ressources mobilisées ». 


\subsection{La contribution par la participation plus visible à l'élaboration du droit de l'Union européenne}

Il est clair que le juge ne peut plus être cantonné au rôle qui lui est classiquement reconnu, celui de «bouche de la loi ». On peut légitimement attendre de lui, ne serait-ce que pour lui permettre d'assurer sa fonction de clarification avec le plus d'efficacité possible, qu'il signale à l'autorité compétente les difficultés récurrentes et, pourquoi pas, qu'il franchisse un pas supplémentaire en recommandant des solutions qu'il pourrait considérer comme adéquates au vu des contentieux dont il est saisi ${ }^{57}$. Dès lors, l'approche inter-institutionnelle suscite deux réflexions principales. La première prend la forme d'un constat : le juge influence déjà le législateur lors de l'élaboration du droit de l'UE par le biais de la technique de la codification. Mais cette démarche nécessairement a posteriori présente un certain nombre de limites. La seconde idée est davantage une piste : le juge pourrait influencer le législateur en lui adressant bien en amont des recommandations, le système français pourrait être source d'inspiration à cet égard.

Une influence directe existe déjà par le procédé de la codification : maintes dispositions trouvent leur origine dans des arrêts que ce soit par exemple en matière de libre circulation ou de citoyenneté. Cette technique présente l'avantage d'inscrire le droit de l'UE dans un cadre global et cohérent en adéquation avec la sécurité juridique et la simplification du droit. Elle permet également de dépasser les clivages politiques entre les États membres et de proposer une solution fondée sur la légitimité du juge. Cela dit, le législateur conserve en la matière la marge de manœuvre inhérente à sa fonction et s'il peut s'aligner purement et simplement sur la position du juge, il peut aussi aller en deçà ou au-delà ${ }^{58}$. Aussi cette reprise en main par le législateur n'est-elle pas, en elle-même, un gage de qualité du droit.

Deux pistes de renforcement de la force de proposition du juge inspirées par le système français peuvent être envisagées.

D’abord, pourrait-on imaginer que le juge européen puisse jouer un rôle de conseiller du pouvoir législatif et exécutif, à l'instar du Conseil d'État? D'après la Constitution française le Conseil d'État est obligatoirement saisi de tous les projets de loi avant leur adoption par le Conseil des ministres et leur dépôt devant le Parlement. Il doit également être saisi des projets d'ordonnance avant leur adoption en Conseil des ministres. De même, les décrets en Conseil d'État ne peuvent être pris ou modifiés qu'après la saisine du Conseil d'État. Dans les autres cas, la consultation reste facultative y compris en matière de propositions de lois. Notons en outre que le Gouvernement peut soumettre une question qui pose un problème juridique particulier pour qu'il puisse être éclairé. Le Conseil d'État comprend donc cinq sections consultatives qui donnent leur avis chacune dans le champ particulier qui leur est dévolu, et pour les textes les plus importants, c'est l'Assemblée générale qui est compétente. Les avis ne sont rendus publics que si l'autorité qui consulte en décide ainsi. Par

57 Dans son Étude de 2016, le Conseil d'État insiste d'ailleurs sur la fonction d'alerte et de suivi que pourrait jouer le juge, op. cit, p. 119 et s. 58 A. Iliopoulou-PenOt, «Réflexions sur la codification de la jurisprudence par le législateur européen », in B. BERTRAND (éd.), L'identité du droit de l'Union européenne, Mélanges en l'honneur de C. BLUMANN, op.cit., p. 187-201. 
conséquent, aux termes de l'article L112-1 du Code de justice administrative, "Le Conseil d'État participe à la confection des lois et ordonnances ». Une transposition de ce système en droit de l'UE serait néanmoins compliquée tant d'un point de vue juridique - encore faudrait-il en effet que tous les États membres soient d'accord pour modifier le Traité - qu'en pratique au vu du nombre réduit des juges, de l'afflux toujours plus important des recours et des nécessités de traductions.

Il conviendrait par conséquent d'explorer la piste a minima : celle qui consiste à donner la possibilité au juge d'attirer l'attention des pouvoirs publics sur les réformes d'ordre législatif, réglementaire ou administratif ${ }^{59}$. Les adaptations seraient alors infimes puisqu'on pourrait aisément imaginer qu'elles soient contenues dans le rapport annuel, sans nécessité de réforme plus profonde.

Tel est le cas du Conseil d'État qui consacre des parties de son rapport public à la contribution des formations contentieuses, d'une part, et consultatives d'autre part, à la simplification du droit ${ }^{60}$. La contribution du juge à l'amélioration de la qualité de la législation est ainsi aisément identifiable y compris par le citoyen.

Tel est également le cas de la Cour de cassation. Aux termes de l'article R431-10 du Code de l'organisation judiciaire : «Le premier président et le procureur général peuvent appeler l'attention du garde des Sceaux, ministre de la justice, sur les constatations faites par la Cour à l'occasion de l'examen des pourvois et lui faire part des améliorations qui leur paraissent de nature à remédier aux difficultés constatées ». Une partie du rapport annuel de la Cour de cassation est dédiée aux « suggestions de modifications législatives ou réglementaires » avec deux sous-parties l'une sur les propositions en matière civile l'autre en matière pénale. Sont ensuite abordés, par ordre thématique, le suivi des suggestions préconisées dans les rapports antérieurs avec un rappel du texte, de la jurisprudence, le contenu de la proposition avec l'objectif recherché sachant que la Cour de cassation pointe finalement les difficultés d'application et/ou les effets qui n'avaient pas été escomptés à l'origine. Un comité de suivi composé des membres du bureau de la Cour de cassation et des membres de la direction des affaires civiles ou pénales selon le cas est institué pour cela.

Le Rapport annuel s'avère par conséquent un instrument utile pour appeler l'attention du législateur et contrôler le suivi des recommandations, ce qui peut d'ailleurs constituer finalement une forme de pression.

\footnotetext{
59 Il en est ainsi pour le Conseil d'État aux termes de l'article L112-3 du Code de justice administrative : « Le Conseil d'État peut, de sa propre initiative, appeler l'attention des pouvoirs publics sur les réformes d'ordre législatif, réglementaire ou administratif qui lui paraissent conformes à l'intérêt général ».

60 Par exemple, p. 67 du Rapport public 2016: «Les contributions de la section du contentieux du Conseil d'État à la simplification du droit revêtent des formes diverses. En opérant une définition claire des requérants susceptibles de se voir reconnaître un intérêt à agir, le Conseil d'État permet aux personnes publiques et à ses cocontractants d'anticiper les risques contentieux. [...] Enfin le Conseil d'État a été amené à accompagner le mouvement de simplification voulu par le législateur et le pouvoir réglementaire en explicitant l'unification des régimes qui avait été opéré en matière de police de l'eau ». Concernant les sections administratives, trois types de contrôles sont exercés permettant de contribuer à la simplification du droit (p. 211) : appréciation juridique (hiérarchie des normes, cohérence et sécurité de la norme juridique), qualité rédactionnelle (respect de l'objectif constitutionnel d'intelligibilité et d'accessibilité de la norme), bonne administration (efficacité et réalisme du dispositif).
} 
Le rôle du juge dans la fabrication du droit de l'UE est réel par l'interprétation, l'application et la vigilance qu'il peut apporter. L'impact juridictionnel du « Mieux légiférer » peut encore être renforcé par son instrumentalisation qui pourrait assurer une visibilité plus grande à la contribution du juge.

Notre conclusion est nécessairement en demi-teinte, mais la tentative de systématisation n'est pas vaine pour autant. Nous avons certes relevé, autant que possible, les limites de l'approche matérielle et de la démarche formelle ; la combinaison des deux ne donne d'ailleurs pas un aperçu plus net de l'impact juridictionnel du « Mieux légiférer ». Pour autant, il y a bien un mouvement, une sorte de dynamique, qui dépasse la seule juridiction européenne, et qui laisse pressentir qu'un tournant s'amorce concernant la visibilité de la contribution du juge à la fabrication du droit. La mouvance du « Mieux légiférer » invite dès lors à réfléchir à la capacité du juge à garantir deux exigences primordiales, dont la portée est renouvelée dans le contexte actuel : la cohérence du droit dans un espace juridique et juridictionnel aux influences nationales, européennes et internationales démultipliées et la légitimité du droit rejaillissant sur la question de la légitimité de l'Union européenne elle-même et de façon circulaire interrogeant la légitimité du juge européen. Au fond, le juge ne serait-il pas le dernier rempart d'une Europe politique en crise? 


\title{
L'IMPACT DU « MIEUX LÉGIFÉRER " SUR L'INTÉGRATION DU DROIT DE L'UNION EUROPÉENNE DANS LES ÉTATS MEMBRES : Le Mieux et le Bien transposer
}

\author{
Emmanuelle SAULNIER-CASSIA ${ }^{1}$
}

\section{Résumé}

Des impacts du « Mieux légiférer » sur l'intégration du droit de l’Union européenne dans les États membres peuvent être identifiés et inciter à des progrès venant prolonger ce « Mieux légiférer ». Assurément, une anticipation renforcée en amont de la procédure de transposition, au moyen d'une implication institutionnelle nationale davantage coordonnée et d'une analyse optimisée des impacts sur les droits nationaux, aurait pour effet un « Mieux transposer ». En outre, une « traçabilité » systématique des opérations de transposition passant par une clarification des méthodes et des outils de transposition et une plus grande cohérence des opérations de transposition, pourrait se traduire par un «Bien transposer ».

\begin{abstract}
Impacts of "Better Regulation" on the integration of EU law in the Member States can be identified and encouraged to make progress in order to prolong "Better Regulation". Certainly, an enhanced anticipation upstream of the transposition procedure, through more coordinated national institutional involvement and an optimized analysis of impacts on national rights, would have the effect of "better transposing". In addition, a systematic "traceability" of transposition operations through clarification of transposition methods and tools and greater consistency of transposition operations could translate into a "Better transposing".
\end{abstract}

$1 \quad$ Professeure de droit public, Université de Versailles-Saint-Quentin - Université Paris-Saclay. 
Le «Mieux légiférer » n'aurait aucun sens si la réception dans les États membres de cette législation de « haute qualité ${ }^{2}$ n'était pas envisagée. Il existe de fait une dépendance entre le « Mieux légiférer » et ce que nous pourrions appeler le «Bien intégrer » qui suppose un «Bien appliquer » dans l'objectif final d'un « Mieux » et «Bien transposer».

Comme on le sait, les États membres doivent appliquer le droit dérivé de l'Union européenne, en l'intégrant dans leurs droits nationaux, en application de l'article 4 TUE et du principe de coopération loyale, soit par la méthode de la transposition pour les directives européennes, soit par une « simple » application s'agissant des autres textes, qui nécessite parfois une adaptation ${ }^{3}$ et le cas échéant des modifications qui peuvent être substantielles dans les droits des États membres. Jean-Claude Bonichot écrivait en 1996 que « les conséquences de cette adaptation sont toujours loin d'avoir été complètement évaluées $»^{4}$. Nous avons plus d'indices depuis et les lacunes des États membres sont très précisément comptabilisées par la Commission qui publie des tableaux d'affichage depuis 1997. Si l'intégration et la mise en œuvre du droit de l'Union dans les droits nationaux s'est progressivement améliorée, la situation n'est tout de même toujours pas idéale et peut évoluer considérablement d'une année l'autre.

Ainsi et alors que l'objectif annoncé dans l'Acte pour le marché unique est de 0,5\%, le tableau d'affichage semestriel du marché unique publié en juillet $2016^{5}$ faisait état d'un déficit de transposition de $0,7 \%$ à l'échelle européenne qui a considérablement augmenté l'année suivante en passant à 1,5\% en juillet 2017 ; sachant que la France est passée dans le même temps de 1 à 1,1\% $\%^{6}$, ce qui peut sembler satisfaisant au regard de l'évolution de la moyenne européenne ou si on compare son taux à d'autres États (par exemple le Luxembourg à 2,2\%), ou encore si l'on a en mémoire les 7,4 \% de la France en 1997 (contre 6,3 \% pour la Communauté dans son ensemble). Plusieurs autres facteurs s'avèrent préoccupants. D'une part, la France n'a pas réussi à stabiliser son taux le plus bas atteint en novembre 2014 $(0,5 \%)$. D'autre part et surtout, le taux en soi du déficit de transposition ne reflète pas complètement l'état du «Bien appliquer » puisque si l'on se réfère aux rapports annuels de la Commission européenne sur le contrôle de l'application du droit de l'Union européenne, et plus particulièrement à l'année 2016, il faut déplorer à l'échelle de l'ensemble des États membres 868 infractions pour retard de transposition en cours ${ }^{7}$, soit une augmentation de 67,5\% par rapport à l'année précédente ${ }^{8}$.

Dès lors, comment croire que les ambitions vertueuses d'un Mieux légiférer prôné tant au niveau européen qu'au niveau national ${ }^{9}$ puissent avoir un impact sur cette intégration du droit de l'Union dans les États membres en vue de sa «meilleure insertion $»^{10}$ ? Si l'on se concentre sur le

\footnotetext{
2 Considérant 2 de l'Accord interinstitutionnel entre le Parlement européen, le Conseil de l'Union européenne et la Commission européenne « Mieux légiférer » du 13 avril 2016, JOUE 12.05.2016, L 132/1.

Conseil d'État, L'adaptation du droit français au droit communautaire, rapport public 1992, EDCE, $\mathrm{n}^{\circ} 44$.

J.-C. BonICHOT, « Le droit communautaire et le droit administratif français », A7DA, 1996, p. 15.

http://ec.europa.eu/internal_market/scoreboard/index_en.htm.

http://ec.europa.eu/internal market/scoreboard/_docs/2017/member-states/2017-france fr.pdf

Commission européenne, Le contrôle de l'application du droit de l’Union européenne, Rapport annuel 2016, 6 juillet 2017, COM (2017) 370 final, p. 30.

Commission européenne, Le contrôle de l'application du droit de l'Union européenne, Rapport annuel 2015, 15 juillet 2016, COM (2016) 463 final, p. 25.

V. notamment le colloque « Mieux légiférer » à l'Hôtel de Lassay le 28 novembre 2014. V. aussi la proposition de loi constitutionnelle de M. J.-F. MANCEL du 21 juillet 2015, n 2999.

10 Conseil d'État, Pour une meilleure insertion des normes communautaires dans le droit national, Paris, Documentation française, 2007,88 p.
} 
niveau européen, il est permis d'avoir quelques doutes, car ce sont finalement les mêmes objectifs qui sont affichés depuis quinze ans. En effet, il faut constater que depuis le Livre blanc sur la gouvernance de 2001 jusqu'au dernier Accord interinstitutionnel « Mieux légiférer » du 13 avril 2016, en passant par la dizaine de textes intermédiaires publiés entre les deux dates, la question de l'impact de cet objectif du « Mieux légiférer » sur l'intégration du droit de l'Union européenne dans les États membres, même si elle a été irrégulièrement prise en considération selon les textes, est inlassablement posée.

Ainsi, dans le chapitre intitulé «Améliorer la réglementation », du Livre blanc sur la Gouvernance de $2001^{11}$, après des considérations relatives à la clarification et la simplification du droit de l'Union, un paragraphe est consacré à « une meilleure application au niveau national » des règles de l'Union européenne. Il est demandé aux États d'« intensifier leurs efforts pour améliorer la qualité de la transposition et de l'application du droit communautaire » avec des propositions très concrètes de moyens pour y parvenir comme la mise en place de « dispositifs de jumelages entre administrations nationales » pour un échange de «bonnes pratiques », la création d' « unités de coordination au sein de l'administration centrale de chaque État membre chargées de l'application du droit communautaire », la familiarisation accrue des tribunaux avec le droit communautaire et la création de mécanismes de médiation pour « améliorer la capacité de règlement des litiges ». Ces propositions sont assorties d'une menace, celle de l'intransigeance de la Commission en cas d'infractions au droit communautaire par les États membres, clairement suggérée dans la communication «Plan d'action «simplifier et améliorer l'environnement réglementaire»»du 5 juin $2002^{12}$, sévérité - bien que relativisée dans la communication de la Commission du lendemain « Gouvernance européenne : mieux légiférer $»^{13}$ - réaffirmée quelques mois plus tard dans la communication « sur l'amélioration du contrôle de l'application du droit communautaire $»^{14}$, puis à nouveau en $2007^{15}$ dans une communication qui a introduit l'idée de « résultats », reprise dix ans plus tard ${ }^{16}$.

\footnotetext{
11 Commission, Gouvernance européenne - Un livre blanc, COM (2001) 428 final, JOCE du 12.12.2001, C 287/01.

12 Commission, COM (2002) 278 final/2. La Commission invite entre autres les États membres à « transposer fidèlement et dans le délai prévu les actes communautaires », à « rationnaliser leurs procédures internes en mettant en place des mécanismes permettant une implication de leurs administrations », à avoir recours à des consultations et analyses d'impact notamment « sur les éventuelles dispositions supplémentaires insérées dans les actes ».

13 Commission, Gouvernance européenne : Mieux légiférer, COM (2002) 275 final/2, non publiée au fOCE. La Commission affirme simplement qu'elle « accordera une attention accrue aux retards pris dans la mise en œuvre des mesures nationales d'exécution » (p. 5).

14 Commission, Communication sur l'amélioration du contrôle de l'application du droit communautaire, COM (2002) 725 final/3 du 20 décembre 2002, non publiée au FOCE : «La Commission entend améliorer le contrôle de l'application du droit communautaire, tout d'abord en accentuant la prévention des infractions. Pour cela, la Commission développera la coopération avec les États membres, notamment en les accompagnant dans la transposition des directives. La Commission continuera également d'endosser son rôle de gardienne des traités à travers la poursuite des infractions. Pour cela, elle s'appuiera sur des critères de priorité relatifs à la gravité des infractions. Par exemple la non-transposition des directives sera considérée comme une infraction grave et la procédure en manquement sera dans ce cas immédiatement engagée. »

15 Commission, Pour une Europe des résultats - application du droit communautaire, COM (2007) 502 final/3 du 5 septembre 2007, non publiée au FOUE. La rigueur souhaitée par la Commission est depuis cette période en outre bien relayée par la Cour, s'efforçant en cas de manquement de statuer rapidement et n'hésitant plus à appliquer des sanctions cumulatives. Mais elle vise avant tout à empêcher autant que faire se peut la saisine de la Cour. La procédure précoce de dialogue entre la Commission et les États membres dite EU-pilot, envisagée dans cette communication de 2007, permet depuis, via une plateforme en ligne dédiée, de résoudre $75 \%$ environ des cas d'infractions. Pour exemple 520 enquête suivant cette procédure ont été lancées par la Commission en 2016 (COM (2017) 370 final, rapport 2016, op. cit., p. 19).

16 Commission, Le droit de l'UE : une meilleure application pour de meilleurs résultats, (2017/C 18/02), fOUE 19 janvier 2017, C 18/10.
} 
On peut trouver en outre des allusions au « Mieux légiférer » dans les communications de mai 2002 sur 《l'Analyse d'impact $»^{17}$, de juin 2002 sur la « Gouvernance européenne : mieux légiférer $»^{18}$, de décembre 2002 sur les «Principes généraux et normes minimales applicables aux consultations engagées par la Commission avec les parties intéressées $»^{19}$. À partir du premier Accord institutionnel «Mieux légiférer » du 16 décembre $2003^{20}$, on peut voir dans les textes successifs de la Commission une démarche « inclusive ${ }^{21}$ volontaire en vue de l'amélioration de l'application du droit de l'Union dans les droits nationaux, jusqu'au dernier Accord interinstitutionnel de 2016 22 . Après avoir posé dès le considérant (2) l'objectif de «faciliter » la transposition et l'« application pratique » d'une « législation de l'Union de haute qualité », qui soit aussi « simple et claire que possible » dans les États membres, l'Accord interinstitutionnel de 2016, comme le précédent de 2003, consacre quelques paragraphes spécifiquement dédiés à la question de l'intégration du droit de l'Union dans les États membres. Plus exactement, le $7^{\mathrm{e}}$ chapitre (§ 41 à 45 ) est relatif à la « mise en œuvre » et l' « application de la législation de l'Union », tandis que le chapitre (non numéroté) de l'Accord de 2003 (§ 32 à 34) était intitulé « Amélioration de la transposition et de l'application ». Au-delà des différences terminologiques et des mises en perspectives différentes, il existe une préoccupation centrale commune entre les deux textes, qui est celle de l'amélioration de la transposition des directives européennes en droit interne, pour éviter les situations d'infractions.

En plus de l'impact du « Mieux légiférer » applicable au strict niveau de l'Union qui aboutirait vraiment à simplifier les textes, à réduire le nombre de directives et à constituer automatiquement un contexte favorable au Mieux et Bien transposer, c'est finalement la perfectibilité de la transposition qui est l'enjeu principal du « Mieux légiférer » s'agissant de son impact sur une meilleure intégration du droit de l'Union en droit interne. Le succès d'un Mieux et d'un Bien transposer, poursuivis depuis tant d'années dépend de l'évolution de deux questions principales qui suivent en quelques sortes une logique chronologique : d'une part, le renforcement de l'anticipation des obligations de transposition (1) et d'autre part, l'établissement d'une «traçabilité » systématisée des opérations de transpositions (2).

L'idée, à travers l'étude de ces deux questions essentielles, est de mesurer la capacité du « Mieux légiférer » à atteindre les objectifs transversaux du Mieux et du Bien transposer, que l'on peut déduire a contrario des deux motifs principaux de manquement en matière de transposition, à savoir les

17 Commission, Communication sur l'analyse d'impact, COM (2002) 0276 final, mai 2002, non publiée au $7 O C E$.

18 Commission, Gouvernance européenne : mieux légiférer, COM (2002) 275 final/2, 6 juin 2002, non publiée au fOCE.

19 Commission, Vers une culture renforcée de consultation et de dialogue - Principes généraux et normes minimales applicables aux consultations engagées par la Commission avec les parties intéressées, COM (2002) 704 final, non publiée au $7 O C E$.

20 Dans cette communication du Parlement européen, du Conseil et de la Commission du 16 décembre 2003, fOUE 31 décembre 2003, C 321/1, les trois institutions rappelaient déjà, dès les engagements et objectifs communs introduisant le texte, qu' : «Elles invitent les États membres à veiller à une transposition correcte et rapide et dans les délais prescrits du droit communautaire dans la législation nationale, conformément aux conclusions de la présidence du Conseil européen lors de ses réunions de Stockholm, Barcelone et Séville ». V. aussi la communication de la Commission de 2005, Améliorer la réglementation en matière de croissance et d'emploi dans l'Union européenne (COM (2005) 97 final) ; la décision 14901/05 sur l'approche interinstitutionnelle commune en matière d'analyse d'impact; la communication de la Commission de 2010, Une réglementation intelligente au sein de l'Union européenne (COM (2010) 0543) et la communication de la Commission de 2012, Pour une réglementation de l'UE bien affutée (COM (2012) 746 final).

21 F. PÉrAldi-Leneuf, « Propos introductif », supra.

22 Cet Accord a été adopté à la suite des communications de la Commission du 19 mai 2015 : Améliorer la réglementation pour obtenir de meilleurs résultats - Un enjeu prioritaire pour l'UE, COM [2015] 215 final et Proposition d'accord interinstitutionnel relatif à l'amélioration de la réglementation, COM [2015] 216 final. 
transpositions tardives et les transpositions incorrectes, ce qui pourrait être traduit de manière positive par une accélération - pour ne pas dire ponctualité - des transpositions ${ }^{23}$ et une exactitude ou fidélité des transpositions du droit de l'Union en droit interne.

\section{1) Une anticipation renforcée en amont de la procédure de transposition pour un Mieux transposer}

L'anticipation peut s'opérer via une amélioration des processus nationaux par une plus grande implication des institutions et acteurs nationaux en charge à la fois de la négociation et de la transposition (1.1) et par l'analyse optimisée des impacts des transpositions sur les droits nationaux (1.2). Elle est suggérée par l'Accord institutionnel, et officiellement encouragée depuis de nombreuses années au niveau national.

\subsection{Par une implication institutionnelle nationale coordonnée}

Alors que dans l'Accord interinstitutionnel de 2003, la question de la transposition est envisagée en soi, de manière totalement autonome, comme un processus en aval de l'adoption de la législation européenne, appartenant aux seuls États membres, sous le contrôle de la Commission et de la Cour, mais sans lien résolu avec l'étape en amont de l'adoption de la législation et alors qu'elle concerne au moins partiellement les mêmes acteurs nationaux, l'Acte de 2016 cite à trois reprises les parlements nationaux, absolument ignorés dans l'Acte de 2003. Autrement dit, le progrès qui semble opéré dans l'Acte de 2016 par rapport à celui de 2003 est d'avoir valorisé l'importance des deux phases - en amont et en aval - de la transposition pour une mise en œuvre réussie du droit de l'Union dans les États membres.

Beaucoup de chemin a déjà été parcouru relativement à l'implication des parlements nationaux, en particulier s'agissant de leur rôle en matière de contrôle de la subsidiarité et de la proportionnalité qui est rappelé d'ailleurs dès le considérant (4) de l'introduction de l'Accord interinstitutionnel, lequel passe néanmoins ensuite largement sous silence la capacité collective des parlements nationaux à bloquer une proposition ${ }^{24}$ de la Commission et n'indique pas ce motif dans les raisons possibles d'un retrait d'une proposition de la Commission (§ 9).

Mais au-delà de la question du contrôle de subsidiarité, l'implication des chambres françaises dans le contrôle des propositions d'actes européens en application de l'article 88-4 de la Constitution française, reste toujours en deçà de celle d'autres États membres ${ }^{25}$, d'une part parce que le nombre de résolutions

23 Au point 42 de l'Accord interinstitutionnel de 2016, « les trois institutions soulignent la nécessité d'une application rapide et correcte de la législation de l'Union dans les États membres » en précisant que le « délai de transposition des directives sera aussi court que possible et, en règle générale n'excédera pas deux ans ».

24 V. E. Saulnier-CAssia, « Article 88-6 de la Constitution » in F. Luchaire, G. Conac, X. PrÉtot, La Constitution de la République française.

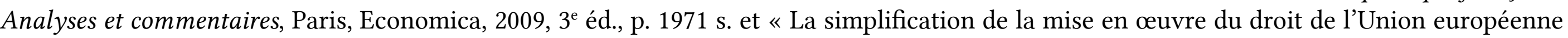
dans le droit français », in D. Bert, M. Chagny, A. Constantin, (éd.), La simplification du droit. Recherches à la confluence de la légistique et de la pratique, coll. Colloques \& Essais, Paris, Institut Universitaire Varenne, 2015, p. 45-46.

25 E. SAulnier, La participation des Parlements français et britanniques aux Communautés et à l'Union européennes, coll. Bibliothèque constitutionnelle et de science politique, tome 108, Paris, LGDJ, 2002, 920 p. 
votées dans chaque chambre est peu important, particulièrement à l'Assemblée nationale, d'autre part parce que les chambres françaises ne peuvent pas, à l'instar d'autres chambres européennes, lier leur gouvernement par un mandat impératif dans le temps du déroulé du processus législatif au niveau européen. L'Accord de 2016 ne fait qu'inciter à une participation parlementaire nationale effective : «Les trois institutions conviennent que les informations fournies aux parlements nationaux doivent permettre à ceux-ci d'exercer pleinement leurs prérogatives en vertu des traités » (§37).

Malgré l'existence depuis 2000 de la base de données IPEX (InterParliamentary EU information eXchange) qui contribue à une coordination utile pour le Mieux transposer, en permettant aux parlements nationaux d'échanger entre eux et le Parlement européen des informations et documents parlementaires, notamment pour mieux suivre les échéances des examens de subsidiarité ${ }^{6}$ et avoir accès aux opinions - résolutions, conclusions, avis politiques - émises par les parlements et les réponses de la Commission ${ }^{27}$, les progrès ne sont pas à la hauteur de l'outil.

Nonobstant, les parlements ne sont pas les seuls concernés par l'anticipation. En effet la transposition se prépare essentiellement dans les Ministères. C'est à ce niveau-là que des efforts supplémentaires doivent être réalisés. C'est le Secrétariat général des affaires européennes (SGAE), service rattaché au Premier ministre, qui est et doit être au cœur de cette mission d'anticipation. C'est ce secrétariat général, en liaison avec la représentation de la France à Bruxelles qui à partir des notes de veille des différents ministères construit un tableau dit de «prospective » énumérant secteur par secteur, les projets de textes en cours au sein de la Commission, tableau mis à la disposition de tous les acteurs pertinents - ministères comme assemblées.

Mais l'anticipation doit aller plus loin pour que les ministères et les assemblées soient prêts à travailler sur le texte de transposition avant même que la directive ne soit publiée au Journal officiel de l'Union européenne (JOUE). Le rapport du Conseil d'État consacré en 2015 aux « Directives européennes : anticiper pour mieux transposer $»^{28}$, a proposé à raison que le processus de transposition débute « dans ce temps mort entre l'adoption de la directive et sa publicité », soulignant le fait qu'il ne sert à rien d'attendre la publication au Journal Officiel et de perdre ainsi plusieurs semaines, voire dans certains cas plusieurs mois alors que le texte est stabilisé. Le rapport remet en cause en cela le calendrier du Guide de bonnes pratiques concernant la transposition des directives européennes édité par le SGAE en 2011 qui, bien qu'il insistât sur l'anticipation, n'envisageait la définition de «l'architecture de la transposition » qu'au moment seulement de la publication de la directive au JOUE ${ }^{29}$.

Mais il faut reconnaître et se féliciter que l'anticipation est parfois voulue par les gouvernements qui proposent eux-mêmes, voire de manière collective, de transposer plus vite que le délai

\footnotetext{
26 Des tableaux très clairs permettent de connaître immédiatement les textes pour lesquels les procédures arrivent à échéance (en rouge) et les nouveaux (en vert) : http://www.ipex.eu/IPEXL-WEB/home/home.do

27 Par un renvoi sur le site de la Commission : http://ec.europa.eu/dgs/secretariat_general/relations/relations_other/npo/

28 Conseil d'État, Directives européennes : anticiper pour mieux transposer, coll. Les Études du Conseil d'État, Paris, La Documentation

française, 2015, p. 35-36, proposition $\mathrm{n}^{\circ} 14$.

29 Guide du 22 décembre 2011 : http://www.fonction-publique.gouv.fr/files/files/textes_de_reference/GBP_transpo_directives.pdf
} 
maximum indiqué par la directive elle-même : c'est le cas par exemple de la quatrième directive anti blanchiment du 24 mai $2015^{30}$ devant être transposée avant le 26 juin 2017 pour laquelle la France a annoncé en conseil des ministres du 22 juillet 2015 son souhait de la transposer avant la fin de l'année. Elle ne l'a finalement été qu'une année plus tard - ce qui reste antérieur de six mois à la date limite - et par voie d'ordonnance ${ }^{31} \ldots$

\subsection{Par l'analyse optimisée des impacts sur les droits nationaux}

Les fiches d'impact ne doivent pas être confondues avec les analyses d'impact, qui sont les premiers outils envisagés dans le chapitre « III - Outils destinés à mieux légiférer de l'Acte interinstitutionnel de 2016, réalisés au niveau de l'Union et concernant ses trois institutions principales. Les États membres sont encouragés par la Commission depuis 2002 à définir eux aussi des normes de consultation et d'analyse d'impact pour la transposition des directives qui leur laissent une marge plus importante pour la mise en œuvre. L'Accord interinstitutionnel ne fait cependant pas référence à ces outils nationaux.

En France, cette pratique existe depuis au moins 2004, concomitamment à la formalisation de la procédure de transposition des directives par la circulaire du 27 septembre $2004^{32}$ - qui n'a d'ailleurs regrettablement pas été actualisée ${ }^{33}$.

Il existe en réalité depuis cette circulaire de 2004 et le Guide des bonnes pratiques de 2011, plusieurs outils, qualifiés de « fiches d'impact » : les fiches d'impact simplifiées dites FIS 1 qui décrivent dans les trois semaines de la transmission par la Commission d'une proposition de directive, le texte et son inscription dans le contexte juridique français. Et les FIS 2, c'est-à-dire les fiches d'impact stratégiques qui sont elles rédigées dans les trois mois et sont plus prospectives en ce sens qu'elles doivent lister les normes à adopter en vue de la transposition.

Suivant cette même périodicité trimestrielle un organe interministériel dit le Groupe à Haut niveau (GHN), composé du Secrétaire Général du Gouvernement (SGG), du SGAE, de membres de cabinets et d'administrations centrales, fait le point sur l'avancement des transpositions. Et un autre groupe, dit le « comité de liaison » réunit SGG, SGAE, cabinet du ministre chargé des relations avec le Parlement et services des commissions parlementaires pertinentes pour préparer la programmation des transpositions quand elles nécessitent l'adoption de textes de nature législative. Une fusion des deux organes serait souhaitable pour assurer davantage de cohérence aux deux étapes, qui sont en fait complémentaires.

30 Directive (UE) 2015/849 du Parlement européen et du Conseil du 20 mai 2015 relative à la prévention de l'utilisation du système financier aux fins du blanchiment des capitaux ou du financement du terrorisme, fOUE du 5 juin 2015, L 141/73. V. le même jour l'adoption du règlement (UE) 2015/847 sur les informations accompagnant les transferts de fonds et abrogeant le règlement (CE) 1781/2006, JOUE du 5 juin 2015, L 141/1. 31 L'ordonnance $\mathrm{n}^{\circ}$ 2016-1635 du $1^{\mathrm{er}}$ décembre 2016 renforçant le dispositif français de lutte contre le terrorisme, JORF du 2 décembre 2016 , transpose la directive et renforce également le dispositif français de lutte contre le blanchiment et le financement du terrorisme en application du règlement (UE) 2015/847, op. cit.

32 Circulaire du 27 septembre 2004 relative à la procédure de transposition en droit interne des directives et décisions-cadres négociées dans le cadre des institutions européennes, FORF du 2 octobre 2004, p. 16920.

33 Elle aurait au moins dû l'être en raison d'informations devenues obsolètes : non seulement le nom du SGCI a été remplacé par celui de SGAE depuis 2005, mais aussi l'objectif en pourcentage de transposition qui a été revu à la baisse... 
L'engagement pris dans l'Accord interinstitutionnel de 2016 « de créer une base de données commune sur l'état d'avancement des dossiers législatifs» (§39) pourrait être un élément d'information en temps réel très utile aux instances nationales pour leur permettre d'anticiper davantage les transpositions.

\section{2) Une « traçabilité » systématisée des opérations de transposition pour un Bien transposer}

Transparence, clarté et cohérence font partie des buts poursuivis par le « Mieux légiférer » et sont en harmonie avec les exigences nationales d'accessibilité, d'intelligibilité et de sécurité juridique. Mais pour que les mots aient une traduction concrète, il faut pouvoir suivre réellement les opérations de transposition en organisant ce que l'on pourrait appeler en reprenant une terminologie de droit matériel européen, une «traçabilité » et un « étiquetage » des mesures de transposition qui passeraient par une clarification des outils et des méthodes disponibles (2.1) et par une cohérence des opérations de transposition (2.2) qui reposent sur le volontarisme des États et un équilibre nouveau à trouver s'agissant de la politisation à géométrie variable du Bien transposer.

\subsection{La clarification nécessaire des méthodes et des outils de transposition}

Alors que la transposition des directives en droit interne est $a$ priori la question la plus « visible » de l'intégration du droit de l'Union dans les droits nationaux, elle est extrêmement « aléatoire ${ }^{34}$ en droit français et dans d'autres États membres également, où la transposition se caractérise sur le plan très matériel des outils, par une grande « fragmentation $»^{35}$. Dans la mesure où comme on le sait le mécanisme de transposition n'impose pas aux États des moyens mais un résultat, l'éventail des mécanismes et des actes nationaux pouvant être utilisés, est large, même s'ils se résument en réalité en France aux deux catégories des lois et des actes réglementaires. Mais la simplicité de ce choix binaire n'est qu'apparente car les actes législatifs et réglementaires peuvent prendre des formes très diverses auxquelles il faut ajouter le procédé des ordonnances. Le choix du support pertinent peut également dépendre des difficultés venant de certaines directives elles-mêmes, selon qu'elles sont très techniques - ce qui a longtemps été le cas et obligeait alors les instances nationales soit à les recopier soit à être en infraction, et à faire se lamenter les assemblées françaises réduites selon elles à un rôle de «moine copiste $»^{36}-$ ou au contraire très transversales, ce qui est plutôt la tendance aujourd'hui. La diversité des types de supports utilisés nuit en tout état de cause, depuis de nombreuses années, considérablement à la visibilité des mesures de transposition et est contradictoire avec l'objectif de l'Accord interinstitutionnel sur le « Mieux légiférer » de « communiquer clairement » au public sur les mesures de transposition.

34 E. SAULNIER-CASSIA, « La simplification de l'intégration et de la mise en œuvre du droit de l'Union européenne dans le droit français », op. cit., p. 40. 35 E. Saulnier-Cassia, « Le droit européen dans l'élaboration de la loi », J.-B. Auby (éd.), L’influence du droit européen sur les catégories du droit public, Paris, Dalloz, 2010, p. 26.

36 «L’Assemblée, quasiment réduite au rôle de moine copiste, [doit] transposer en droit interne, avec un enthousiasme mitigé, un nombre de plus en plus important de directives communautaires élaborées selon des modalités rien moins que transparentes » : M. PEZET in Assemblée nationale, rapport d'information $\mathrm{n}^{\circ} 3255$ du 24 février 1993, p. 9. 
Selon la marge de manœuvre réelle laissée aux États membres et l'urgence à transposer, le choix de l'outil se pose donc. La catégorie législative est celle qui offre le plus de diversité, aux côtés de la catégorie réglementaire et de la particularité des ordonnances.

La catégorie réglementaire ne pose pas de difficulté particulière. Elle permet en général de transposer des directives techniques, qui n'entraînent pas de réformes de grande ampleur. Elle répond généralement aux objectifs de transparence et de clarté, en ne dissimulant pas l'opération de transposition, c'est-à-dire en indiquant explicitement cet objet dans l'intitulé du décret ${ }^{37}$.

Dans la catégorie législative, le choix d'une loi classique n'est pas sans poser de difficultés. En effet, les lois transposant les directives européennes n'indiquent pas toujours leur objet en tant que tel dans leurs intitulés. En outre, certaines lois transposent les directives en leur entier, d'autres ne transposent que quelques dispositions - avec parfois des éléments de règlements qui en théorie n'ont pas besoin d'être transposés -, d'autres encore plusieurs directives. Ainsi, un nombre non négligeable de transpositions est opéré par étapes, comme cela a été le cas pour la très importante réforme en matière de commande publique ${ }^{38}$, qui a entraîné l'adoption d'une grande variété d'actes sur plus d'une année $^{39}$, en dépit de la volonté affichée du ministre de l'Économie et des Finances de «simplifier » les procédures et d'opérer les transpositions dans des « corpus juridiques clairement identifiés $»^{40}$.

\section{Comment dès lors s'y retrouver?}

Parmi les lois dédiées aux transpositions de directives, existent les lois dites portant diverses mesures d'adaptation au droit de l'Union européenne (DDADUE), ex DDAC (adaptation au droit communautaire) mais qui ont l'inconvénient d'être faussement transparentes. En effet, si l'objectif de transposition est clairement indiqué, il est difficile de savoir quelles sont les directives concernées ou en tout cas de tracer la réelle adaptation interne qui a été nécessaire, dans la mesure où elles transposent généralement plusieurs textes - et parfois partiellement - ce qui leur a valu le qualificatif assez mérité de « voiture-balai législative $»^{41}$. C'est souvent l'urgence due à l'approche du délai

37 V. par exemple le décret n 2016-622 du 19 mai 2016 portant transposition de la directive 2014/17UE du Parlement européen et du Conseil du 4 février 2014 sur les contrats de crédit aux consommateurs relatifs aux biens immobiliers à usage résidentiel et modifiant les directives 2008/48/CE et 2013/36/UE et le règlement (UE) $n^{\circ}$ 1093/2010, FORF du 20.05.2016. Ce texte a néanmoins depuis été abrogé en raison de la réforme du Code de la consommation entrée en vigueur au $1^{\mathrm{er}}$ juillet 2016.

38 Directive 2014/23/UE du Parlement européen et du Conseil du 26 février 2014 portant sur l'attribution des contrats de concession et deux directives concernant la passation des marchés publics, abrogeant les précédentes directives 2004/17/CE et 2004/18/CE ; v. aussi les directives 2014/24/UE pour les marchés « classiques » et 2014/25/UE pour les marchés passés par des entités opérant dans les secteurs de l'eau, de l'énergie, des transports et des services postaux.

39 Notamment : le décret $\mathrm{n}^{\circ}$ 2014-1097 du 26 septembre 2014 portant mesures de simplification applicables aux marchés publics a transposé de manière accélérée certaines dispositions et modifié le code des marchés publics ; la loi n 2014-1545 du 20 décembre 2014 relative à la simplification de la vie des entreprises et portant diverses dispositions de simplification et de clarification du droit et des procédures administratives a autorisé le Gouvernement à prendre par voie d'ordonnance, les mesures de transposition de niveau législatif des directives « Marchés » ce qui a été fait par l'ordonnance du 23 juillet 2015 ; la loi du 7 août 2015 pour la croissance, l'activité et l'égalité des chances économiques autorise le gouvernement à transposer par ordonnances la directive contrats de concession, ce qui a été fait par l'ordonnance du 29 janvier 2016 relative aux contrats de concession; différents décrets sur les seuils des marchés publics (2015-1163 du 17 septembre 2015 et 30 décembre 2015$)$ et sur les règles de passation de contrats de concession (2016-86 du $1^{\text {er }}$ février 2016), puis l'adoption du Code...

40 Allocution de clôture du colloque du ministère de l'Économie et des Finances, Marchés publics et concessions. Quelle transposition en droit français?, 12 mars 2014, p. 5 : http://www.economie.gouv.fr/files/files/directions_services/daj/marches_publics/colloque/discours-cloture-P-Moscovici12mars2014.pdf.

41 L'expression a été employée par P. Lefebvre, au cours du débat au Sénat du $1^{\text {er }}$ février 2000 sur la discussion du projet de loi portant diverses dispositions d'adaptation au droit communautaire dans le domaine des transports : «'voiture balai législative' regroupant plusieurs textes européens en souffrance que le Parlement est chargé d'adopter en urgence pour éviter une condamnation de notre pays ». 
limite de transposition, voire même de son large dépassement, qui justifie l'addition de plusieurs directives, souvent sans aucun rapport thématique entre elles ${ }^{42}$, dans le cadre d'une procédure accélérée. Si la pratique est regrettable, elle est néanmoins de moins en moins utilisée, ce que l'on doit approuver, d'autant plus que ce mécanisme ne garantit pas toujours une transposition rapide et nécessite souvent des actes réglementaires complémentaires ${ }^{43}$.

Les ordonnances sont également le plus souvent utilisées quand il y a urgence à transposer ${ }^{44}$, sans qu'elles non plus, n'offrent une garantie quant au respect des délais ${ }^{45}$, car elles nécessitent l'autorisation et la ratification des assemblées et la signature du Président de la République.

Cette diversité de textes et de vocabulaire contribue donc à rendre confuse la visibilité de l'obligation de transposition, alors que désormais en plus de l'indication de l'obligation de notification à la Commission des mesures nationales de transposition, les directives contiennent le plus souvent une disposition relative à l'obligation pour les États membres de référencement explicite dans les textes nationaux de transposition ${ }^{46}$, ce qui va dans le sens de la recommandation d'un meilleur suivi de l'opération de transposition dans la logique du « Mieux légiférer ». Il convient donc d'insister sur la nécessité de systématiser en France la «nomination » des textes, quel que soit le support retenu, afin que soit indiquée clairement dans des intitulés simplifiés l'opération de transposition d'une directive - par exemple loi portant transposition de la directive $\mathrm{x}$ en matière de $\mathrm{y}$ - à la suite d'une procédure dédiée d'adoption par voie accélérée, qui garantirait le non-dépassement des délais.

À cette clarté, qui non seulement rendrait plus lisible et accessible l'état du droit pour les personnes ou entités concernées, mais qui faciliterait aussi le contrôle de la Commission qui réceptionne les notifications des mesures de transposition, il faut ajouter la cohérence des opérations de transpositions à l'occasion desquelles les États membres ne doivent plus user de leur marge de manœuvre pour sous ou sur-transposer.

42 V. par exemple la loi n² 2015-1567 du 2 décembre 2015 portant diverses dispositions d'adaptation au droit de l'Union européenne dans le domaine de la prévention des risques (JORF du 3.12.2015, p. 2299) qui transpose via la procédure accélérée (engagée par le gouvernement le 15 juillet 2015) cinq directives datant de 2012 à 2015 allant des accidents impliquant des substances dangereuses aux OGM en passant par les équipements marins et autres et qui en même temps adapte le droit national à trois règlements !

43 V. par exemple la loi $\mathrm{n}^{\circ} 2015-993 \mathrm{du} 17$ août 2015 portant adaptation de la procédure pénale au droit de l'Union européenne (FORF du 18.08.2015, p. 14331) transposant trois décisions-cadre JAI du Conseil de 2008 et 2009. Malgré la procédure accélérée, il a fallu plus d'un an pour l'adoption de cette loi car il y a eu un désaccord au niveau de la commission mixte paritaire et le Conseil constitutionnel saisi par des sénateurs a rendu une décision de conformité partielle (près de 30 articles déclarés non conformes à la Constitution car adoptés par voie d'amendements alors qu'ils n'avaient « pas pour objet » de transposer une directive européenne et donc ne présentaient pas de «lien direct » avec le texte déposé....). En outre, deux décrets (du 26 février 2016) ont été nécessaires pour compléter la loi.

44 V. par exemple l'ordonnance 2016-623 du 19 mai 2016 portant transposition de la directive 2014/40/UE sur la fabrication, la présentation et la vente des produits du tabac et des produits connexes, dont la publication au JORF ( ${ }^{\circ} 116 \mathrm{du} 20$ mai 2016) a eu lieu le jour de l'expiration du délai de transposition fixé par la directive.

45 V. par exemple l'ordonnance 2016-526 du 28 avril 2016 portant transposition de la directive 2014/61/UE du Parlement européen et du Conseil du 15 mai 2014 relative à des mesures visant à réduire le coût du déploiement de réseaux de communications électroniques à haut débit (JORF $\mathrm{n}^{\circ} 101 \mathrm{du} 29$ avril 2016) a dépassé le délai maximum de transposition qui était fixé au $1^{\text {er }}$ janvier 2016, tout comme l'ordonnance $\mathrm{n}^{\circ} 2016-1823$ du 22 décembre 2016 portant transposition de la directive 2014/26/UE du Parlement européen et du Conseil du 26 février 2014 concernant la gestion collective du droit d'auteur et des droits voisins et l'octroi de licences multiterritoriales de droits sur des ouvres musicales en vue de leur utilisation en ligne dans le marché intérieur (FORF n 298 du 23.12. 2016) dont le délai maximum était fixé au 10 avril 2016.

46 V. par exemple la directive 2014/40/UE sur la fabrication, la présentation et la vente des produits du tabac et des produits connexes, fOUE du 29.04.2014, L 127/1, indique en son article 29, al. 2 : «Lorsque les États membres adoptent ces dispositions, celles-ci contiennent une référence à la présente directive ou sont accompagnées d'une telle référence lors de leur publication officielle. Elles contiennent également une mention précisant que les références faites, dans les dispositions législatives, réglementaires et administratives en vigueur, à la directive abrogée par la présente directive s'entendent comme faites à la présente directive. Les modalités de cette référence et la formulation de cette mention sont arrêtées par les États membres ». 


\subsection{La cohérence des opérations de transposition}

La transposition des directives suppose consubstantiellement l'existence d'une marge d'appréciation des États membres pour intégrer les directives dans leurs droits internes. La marge de manœuvre des États peut être vue comme un frein à l'application du droit de l'Union ou au contraire comme un moyen de mieux intégrer les normes. Habitués à constater les abus des États membres, les européanistes ont tendance à se méfier de l'utilisation par les États de cette marge pour souvent aller en deçà des obligations de transposition, ou en produisant des harmonisations minimales. Un phénomène moins connu et à l'opposé des transpositions a minima ou incomplètes - mais qui a également pour effet d'introduire et laisser « subsister des différences de mises en œuvre » des directives «par les différents États membres $\gg^{47}$ - est la pratique des sur-transpositions. Ce phénomène conduit à s'interroger plus généralement sur la politisation des transpositions qui est finalement au cœur du « Mieux légiférer ».

Le phénomène dit de «sur-transposition » est connu dans le jargon communautaire par son expression anglaise de «gold-plating » qui est également utilisée pour qualifier la législation européenne quand elle va au-delà de ce qui était attendu au regard de la base juridique utilisée.

Outre la demande déjà mentionnée, faite aux États au point 41 de l'Accord interinstitutionnel de 2016, d'être transparents lorsqu'ils adoptent les mesures nationales de transposition ou d'application du droit de l'Union - « communiquer clairement à destination de leur public » (sic) «sur ces mesures » - il est requis des États qu'ils ne dissimulent pas la réalité de leur action de transposition ou de mise en œuvre du droit de l'Union. Si l'Accord n'interdit pas formellement les pratiques de sur-transposition, il exige que lorsque « des États membres décident d'ajouter des éléments qui ne sont aucunement liés à cette législation de l'Union », les « ajouts » soient « identifiables » soit dans l'acte de transposition, soit dans des «documents associés» (pt 43). L'exigence est parfaitement légitime et participerait pleinement au succès du « Mieux légiférer », car cette étape finale est aussi capitale que celle de départ, de rédaction des actes.

Il est en outre envisagé au point 45 de l'Accord que la Commission intègre les références à ces précisions ou informations à son rapport annuel sur l'application de la législation de l'Union. L'on peut de manière incidente regretter qu'il ne soit pas envisagé de modifier la périodicité de ce rapport, en le rendant par exemple semestriel, ce qui correspondrait à la demande d'accélération des transpositions et qui mettrait peut-être une pression plus grande sur les États.

La question des sur-transpositions n'a pas été ignorée au plan national. En France, le Conseil d'État a ainsi constaté dans un rapport de 2015 qu' «A chaque étape, l'autorité compétente a l'occasion, qu'elle saisit assez souvent, d'ajouter des dispositions que n'imposait pas la norme supérieure $»^{48}$ et

\footnotetext{
47 J.-S. Bergé, L'application du droit national international et européen. Approche contextualisée des cas de pluralisme juridique mondial, Paris, Dalloz, 2013, p. 37.

48 Conseil d'État, Simplification et qualité du droit, Étude annuelle 2016, coll. Études et documents, Paris, La documentation française, 2016, 256 p., p. 54.
} 
a estimé qu' ' aux crispations sur la capacité à absorber le droit de l'Union (retards de transposition) et parfois à s'y conformer sur le fond (débat sur les OGM), s'ajoutent désormais des controverses sur les sur-transpositions (normes adoptées par les États à l'occasion de la transposition, mais sans y être contraints par celle-ci) $»^{49}$.

En effet, crispations et controverses se sont manifestées en France devant les chambres parlementaires. Le Sénat et l'Assemblée nationale ont réagi au moment de l'examen de la proposition d'Accord interinstitutionnel suivant la procédure de l'article 88-4 de la Constitution, en adoptant rapport et résolutions critiquant les allusions au phénomène de sur-transposition et s'accordant pour souhaiter encore et toujours que soit renforcé le dialogue avec les parlements nationaux tout au long de la procédure législative. Au Sénat, la disposition a posé problème en ce qu'elle a été perçue comme revenant à demander aux parlements nationaux de limiter leur pouvoir législatif et a été perçu comme contradictoire avec la nature des directives qui normalement laissent aux États le choix de la forme et des moyens ${ }^{50}$. À l'Assemblée nationale, la réaction a été identique, la chambre basse estimant que « la transposition des directives en droit interne relève pleinement des États membres » et partant s'est fermement « opposée aux dispositions contenues dans la proposition d'accord visant à encadrer la sur-transposition des directives », y voyant quant à elle une contrariété avec le principe de subsidiarité51.

Si la réaction des assemblées n'est pas surprenante, elle ne peut être pleinement approuvée, car elle risque d'introduire une confusion entre la politisation nécessaire de l'obligation de transposition et la dépolitisation indispensable de l'opération stricto sensu des transpositions.

Le volontarisme des États pour réussir le « défi de la mise en œuvre $»^{52}$ du droit de l'Union européenne est évidemment essentiel à sa réussite. Mais les intérêts des États membres ne sont pas uniformes. Très peu d'États ont une « doctrine » au regard de l'obligation de transposition. Et il faut distinguer le principe lui-même de l'obligation de la mise en œuvre en soi de l'opération de transposition.

L'idée du Conseil d'État selon laquelle le « respect de l'exigence de transposition » doit être un « objectif politique $»^{53}$ peut être approuvée, mais à condition de bien circonscrire cette politisation au respect de l'exigence de transposition, non à la transposition elle-même. La politisation principale doit prendre place au niveau européen et au moment de la négociation. Ensuite, il s'agit de remplir une obligation certes d'origine constitutionnelle et européenne, et dont le respect résulte d'une volonté politique, mais dont l'accomplissement matériel doit être dépolitisé.

\footnotetext{
$49 \quad$ Ibid., p. 85

50 Sénat, Rapport $\mathrm{n}^{\circ}$ 84, commission des affaires européennes, MM. BIzET et SuTOuR, sur la proposition d'accord interinstitutionnel relatif à l'amélioration de la réglementation, 15 octobre 2015, 32 p., p. 14.

51 Assemblée nationale, résolution européenne sur l'accord interinstitutionnel « mieux légiférer », 3 janvier 2016, TA n 655. La même position est exprimée dans la résolution du Sénat du 20 novembre 2015, TA 85.

52 H. OberdoRfF, «À la recherche d'un meilleur ordre juridique pour l'Union européenne » in F. PÉrALDi-LENEUF, S. DE LA RosA (éd.),

L'Union européenne et l'idéal de la meilleure législation, Paris, Pedone, 2013, p. 38.

53 Conseil d'État, Directives européennes : anticiper pour mieux transposer, Paris, La documentation française, 2015 , p. 43.
} 
Le respect de cette obligation, qui passe par l'interdiction des sur-transpositions ne peut que contribuer à respecter les objectifs de clarté, de transparence, de rapidité, mais cela ne doit pas pour autant empêcher les États membres de compléter les transpositions stricto sensu par des réformes en profondeur de certains aspects de leurs droits, quand cela est nécessaire.

Le constat déjà opéré par Jacques Chevallier selon lequel « Mieux légiférer » impliquerait de 《réévaluer en permanence le stock des textes existants ${ }^{54}$ peut et doit être appliqué à l'impact du «Mieux légiférer » sur l'insertion du droit de l'Union dans les droits nationaux et donc du Mieux et Bien transposer. Les États membres doivent en effet s'appliquer à la fois à transposer rapidement le droit de l'Union, tout en réévaluant constamment l'adéquation à la fois de leur législation au droit de l'Union, et de la cohérence de leur droit national pouvant impliquer des réformes de plus grande ampleur. C'est ainsi seulement que Mieux et Bien transposer se conjugueront efficacement avec le « Mieux légiférer ».

Le dépassement du Mieux légiférer et du Mieux et Bien transposer en cas de retrait d'un État membre : dé-transposer ou ne pas dé-transposer le droit de l'Union?

La question de la sortie d'un État membre de l'Union qui était théorique jusqu'au référendum britannique du 23 juin 2016 a suscité encore des interrogations autour de la procédure de la sortie elle-même, et pourrait en susciter d'autres sur les conséquences pour les droits nationaux d'une sortie effective. L'on sait bien que la progressive et « assez radicale » « européanisation des droits nationaux $»^{55}$ est venue transformer la plupart d'entre eux. Le doyen Cornu citant Carbonnier a même écrit que « l'ingestion (et l'indigestion) d'un corps d'importation s'aggrave d'un risque de dégénérescence pour le système juridique français » et allant jusqu'à considérer que le processus «prospère au mépris du génie du droit français $»^{56}$, ignorant en cela les influences réciproques, voire l'inspiration que le droit de l'Union trouve souvent de manière féconde dans certains droits nationaux et qui ont fait du Royaume-Uni à l'instar d'autres États membres de l'Union un «État intégré $\gg^{57}$. De nombreux responsables politiques britanniques ont d'ailleurs utilisé avec profit cette idée de dégénérescence du droit britannique due au(x) droit(s) européen(s).

54 J. Chevallier, « Peut-on rationnaliser la production du droit? », in F. PÉRALDi-Leneuf, S. DE la Rosa (éd.), L’Union européenne et l'idéal de la meilleure législation, op. cit., p. 20.

55 H. OberDoRFF, «L'européanisation du droit des États membres de l'Union européenne. Réflexion sur une énigme » in: L’Union européenne : Union de droit, Union des droits. Mélanges en l'honneur de Philippe Manin, Paris, Pedone, 2010, p. 712.

56 G. Cornu, Droit civil. Introduction. Les personnes. Les biens, $12^{\mathrm{e}}$ éd., Paris, Montchrestien, coll. Domat, 2005 , § 263.

57 E. Dubout, B. NABLI, Droit français de l'intégration européenne, Paris, LGDJ, coll. Systèmes, 2015, p. 8. 
Plus concrètement, tout en devant continuer à agir comme un membre à part entière de l'Union européenne ${ }^{58}$ en attendant l'expiration du délai ouvert par l'activation de la clause de l'article 50 TUE et conduisant - le cas échéant - au retrait effectif, les juristes britanniques doivent organiser le basculement d'un droit interne pénétré de normes européennes à un droit interne expurgé de ces mêmes normes pour que le retrait ait un sens.

L'action juridiquement indispensable est l'abrogation du European Union Act adopté en 1972 - alors appelé European Communities Act - qui est la loi qui a légalement permis au droit de l'Union d'avoir la primauté en droit britannique et à tous les actes adoptés par les institutions de l'Union d'avoir le même effet que s'ils avaient été adoptés par le Parlement britannique lui-même ${ }^{59}$. Le droit européen n'existe et n'a d'effets au Royaume-Uni que parce que sa force juridique est autorisée par cette loi britannique de valeur constitutionnelle. Toutefois, le European Union (Withdrawall) Act - un temps appelé Great Repeal Bill - doit tout autant abroger l'ECA, que confirmer l'application d'une partie au moins du droit de l'Union, en le transformant en droit purement britannique. La possibilité d'examiner quelles législations ou réglementations devront être modifiées ou abrogées est renvoyée à plus tard. Quelle ironie !

Certes sur le plan pratique, cela permet d'éviter des vides et une certaine insécurité juridiques ${ }^{60}$ qui ne manqueraient pas de surgir. Mais cela pose la question de la compétence de la Cour qui, si elle a été longtemps balayée d'un revers de manche par le Premier ministre et son ministre en charge de la sortie de l'Union, ne peut pas être totalement exclue à partir de l'adoption de la loi d'abrogation, au moins pour les questions antérieures à l'entrée en vigueur de cette loi. Des condamnations en manquements pourraient être prononcées pour des situations passées dans lesquelles le Mieux et le Bien transposer n'auraient pas été respectés...

\footnotetext{
58 Comme y a insisté un rapport de la Chambre des Lords un mois après le référendum : HousE OF LoRDs, European Union Committee, Scrutinising Brexit : the role of Parliament, 22 juillet 2016, HL Paper 33, pt. 34 p. 10 : « The Committee continues to scrutinise European Union documents, in accordance with its terms of reference, albeit with a particular focus on the significance of documents in the context of UK withdrawal. In so doing, we are responding in part to the fact that the UK will remain a full member of the EU until any withdrawal agreement comes into force, and that it also continues to be bound by EU law ». V. aussi l'audition de David Davies le 12 septembre 2016 par Select Committee on the European Union de la House of Lords : http://data.parliament.uk/writtenevidence/committeeevidence.svc/evidencedocument/ european-union-committee/brexit-parliamentary-scrutiny/oral/38223.html

59 E. Saulnier, La participation des Parlements français et britannique aux Communautés et à l'Union européennes, op. cit., p. 80. Du même auteur, ouvrage à paraître en 2018 sur le Brexit, coll. Systèmes, LGDJ.

60 D'autres lois devenant sans objet (European Parliamentary Elections Act 2002, du European Parliament (Representation) Act 2003, du European Union (Amendment) Act 2008 et du European Union Act 2011) doivent également être abrogées et d'autres doivent être remplacées (notamment en matière de pêche et de sureté nucléaire) pour éviter autant que faire se peut les vides juridiques.
} 
Chapitre 2

APPROCHES SECTORIELLES DE LA MISE EN đUUVRE DU « MIEUX LÉGIFÉRER 》 



\title{
« MIEUX LÉGIFÉRER » \\ L'EXEMPLE DES NOUVELLES TECHNOLOGIES DE L'INFORMATION
}

\author{
Jean-Claude BonICHOT ${ }^{1}$
}

\section{Résumé}

Cette communication vise à déterminer les difficultés auxquelles le juge est confronté dans le domaine des nouvelles technologies de l'information ainsi que les solutions qu'il peut apporter. Il s'agit également de s'interroger sur la contribution du juge à l'amélioration de la qualité du droit de l'Union européenne.

\section{Abstract}

This paper aims at determining the difficulties with which the judge is confronted in the field of the new information technologies as well as the solutions which he can bring. The question is also about the contribution of the judge to the quality of European law.

On ne peut manquer de relever l'actualité du sujet retenu par le Centre d'Études et de Recherches Internationales et Communautaires (CERIC) de l'Université d'Aix-Marseille. En effet, le Conseil d'État, mon corps d'origine, vient de consacrer son étude annuelle 2016 au thème « simplification et qualité du droit $»^{2}$, après ses rapports de 1991 et 2006 consacrés à la sécurité juridique et à la complexité du droit'. Il s'était penché, en 2013, sur le «droit souple »4, idée prémonitoire puisqu'il y a juste quelques mois l'assemblée, puis la section du contentieux, ont défini son nouveau statut contentieux en faisant preuve d'un réalisme qu'on ne peut que saluer. En effet, le Conseil d'État a considéré que « les avis, recommandations, mises en garde et prises de position adoptés par les autorités de régulation dans l'exercice des missions dont elles sont investies » peuvent être attaqués pour excès de pouvoir lorsqu'ils ont des effets juridiques certains'. Il s'agit, en effet, de droit, et d'un

Juge à la Cour de justice de l'Union européenne.

Étude annuelle 2016 du Conseil d'État, Simplification et qualité du droit, La documentation Française, 2016, 256 p.

De la sécurité juridique, Études et documents du Conseil d'État, 1991 ; Sécurité juridique et complexité du droit, Études et documents du Conseil d'État, 2006.

Le droit souple, Étude annuelle 2013.

CE ass. 21 mars 2016, Société Fairvesta International Gmbh et autres, et Société Numéricable, AJDA, 2016, p. 717, chron. ; L. DuTHEILLET DE LAmothe Et G. Odinet, RFDA, 2016, p. 497, conclusions S. von Coester et p. 506, conclusions V. Daumas ; CE sect. 13 juillet 2016 , Société GDF Suez, AfDA, 2016, p. 2119, note F. MELlERAY. 
droit très important en pratique et qui doit être contrôlé. Cela n'est pas sans rappeler la jurisprudence fort pragmatique de la Cour de justice tant pour ce qui concerne le recours en annulation que le renvoi préjudiciel.

Comment se situe le juge par rapport à cet impératif de mieux légiférer ? Le juge n'est pas le législateur. Mais son rôle ne se limite pas à la seule application du droit ; il contribue de manière décisive à sa création. Il est aussi fort bien placé pour constater les insuffisances de la législation, ses lacunes éventuelles et même parfois ses contradictions. Il est au centre des questions de cohérence législative : il doit souvent se demander si tel concept (ou simplement définition) qui figure dans telle partie de la législation doit recevoir le même contenu dans une autre.

Cette question peut se poser pour de nombreuses notions, ou termes, ou expressions. Deux exemples : la notion majeure d' "établissement » doit-elle recevoir le même contenu en matière fiscale et dans celle des nouvelles technologies de l'information (NTI) ou encore en droit du travail ? Ou au contraire faut-il raisonner seulement à l'intérieur de l'une ou l'autre matière en fonction de ses caractéristiques propres, de sa logique à elle ? Dans un même domaine comme celui des NTI faut-il toujours donner le même sens à l'expression « communication au public » qui est utilisée dans différentes directives?

Le domaine des technologies de l'information est un champ d'étude intéressant du point de vue de la légistique. D'abord parce que c'est un domaine d'une importance considérable dans le monde d'aujourd'hui. Ensuite parce que c'est un domaine techniquement compliqué et difficile. En troisième lieu car il est transversal : j'étais intervenu ici même il y a un an et j'avais tenté de montrer que les technologies de l'information concernent aussi bien la protection des données personnelles que le commerce électronique (et donc la protection des consommateurs), la propriété intellectuelle et la procédure civile ou pénale ${ }^{7}$ Enfin parce qu'il est extrêmement évolutif. La question se pose donc de savoir comment appliquer des textes adoptés il y a quelques années alors que le contexte social comme technique peut avoir changé.

Je vais me placer délibérément du côté du juge : quelles sont les difficultés auxquelles il est confronté? Comment s'en sort-il ? Quelle peut être sa contribution à l'amélioration de la qualité du droit?

\section{1) Commençons par constater qu'aussi parfaite soit-elle, une législation ne peut ni tout prévoir ni tout régler.}

Elle ne le doit d'ailleurs pas. Une législation doit trouver un équilibre entre précision et souplesse. C'est la condition absolue de son applicabilité et de sa longévité. La manière dont a été écrit le code civil français en est un excellent exemple et gageons que les textes qui l'ont modifié sur des questions

\footnotetext{
E. von Bardeleben, F. Donnat, D Siritzky, La Cour de justice et le droit du contentieux européen, La Documentation française, 2012, p. 170 et 218 .

On en retrouve les grandes lignes dans mon article : « La Cour de justice de l'Union européenne et les nouvelles technologies de l'information : vers une Cour 2.0 ? », Les Petites Affiches, 15.03.2016, n 53 p. 8.
} 
essentielles ne dureront pas aussi longtemps... Pour ce qui est du droit de l'Union, il est impératif de tenir compte du système particulier dans lequel il est élaborés. Je ne vais pas insister sur cet aspect qui est abordé par ailleurs, mais il faut garder cela à l'esprit.

Contrairement à ce qui est souvent dit et répété dans un contexte, il est vrai, assez antieuropéen, la législation de l'Union européenne est dans son ensemble une bonne législation. Elle n'a sans doute pas grand-chose à envier à la législation française, si ce n'est les conditions dans lesquelles elle est mise à la disposition du public ${ }^{9}$.

En premier lieu, c'est une législation généralement rédigée de manière correcte, qu'il s'agisse des directives, des règlements ou des autres actes obligatoires ou pas.

Le Traité exige que les actes des institutions soient motivés. Cette obligation concerne aussi bien les actes généraux que les actes individuels.

Les actes législatifs et autres que nous avons à appliquer comportent un préambule, le plus souvent très détaillé. Il retrace en général l'histoire de la législation en cause et explicite le sens des différentes dispositions du texte. La plupart du temps figure au début du texte la définition des termes employés.

Les préambules sont très largement utilisés par la Cour de justice pour l'interprétation des textes. À cet effet ils sont largement cités dans les arrêts.

Dans certains domaines, le contenu de l'acte peut être inévitablement technique. Mais c'est une véritable utopie de croire que l'on peut mettre tous les textes juridiques à la portée du plus grand nombre. De ce point de vue, il n'y a pas de différence avec les législations nationales, notamment française. Vouloir à toute fin rédiger des textes juridiques dans le langage commun aboutit le plus souvent à des rédactions longues et absconses, ce qui ne veut pas dire qu'il faille systématiquement se complaire dans les termes de la technique juridique. Il faut seulement écrire le plus simplement possible.

En deuxième lieu, la législation communautaire se caractérise par une assez grande stabilité, contrairement, par exemple, à la législation française. Ce défaut de la législation française est d'ailleurs pointé une fois de plus par le Conseil d'État. Il est rare en droit de l'Union qu'une directive soit abrogée avant même d'être entrée en vigueur ; c'est un peu moins rare en France...

Cette stabilité tient en grande partie à la longueur du processus législatif, elle-même due à sa complexité et à la difficulté corrélative de modifier la législation.

G. Tuts, « Améliorer la qualité rédactionnelle : une approche lucide et enthousiaste », A Man for all Treaties, Liber amicorum en l'honneur de fean-Claude PIRIS, Bruylant 2012, p. 565.

Il est regrettable que l'on n'explique pas mieux à nos concitoyens que les fameuses « règles européennes » que l'on trouve si excessives remplacent en réalité, dans chaque domaine, par une règle commune les 28 règles qui autrement, de toute façon, existeraient et divergeraient. C’est donc une simplification majeure que réalise mécaniquement la législation européenne ! 
Il est procédé périodiquement à une « codification » des textes qui ont fait l'objet de nombreuses modifications. Cette « codification » qui est, en réalité, une consolidation n'a pas grand-chose à voir avec la codification française qui est, il est vrai, un processus dont notre pays a l'habitude, qui est bien rôdé et donne de très remarquables résultats. On en a un excellent exemple avec le code des relations entre le public et l'administration ${ }^{10}$.

La codification communautaire a souvent pour objet d'incorporer au texte la jurisprudence à laquelle il a donné lieu. Le règlement $1408 / 71^{11}$, sur la sécurité sociale et est un exemple.

On peut, en revanche, regretter les conditions de mise à disposition de la législation communautaire qui me semble moins accessible que la législation française qui bénéficie avec Légifrance, d'un instrument incomparable.

Il est vrai que la jurisprudence joue, comme partout, un rôle important, mais il n’y a là rien que de très normal.

Il ne faut donc pas considérer que lorsque le juge doit intervenir, c'est que la loi est mal faite.

À titre d'illustration je prendrai deux exemples dans la jurisprudence de ces derniers mois.

La Cour de justice a eu à s'interroger une nouvelle fois sur la notion de « communication au public » dans le cadre de la protection du droit d'auteur dans le contexte d'Internet. Le droit de l'Union, et en particulier la directive sur le droit d'auteur dans la société de l'information ${ }^{12}$, prévoient le droit exclusif de l'auteur de permettre la communication au public de leurs œuvres. Comment appliquer ce principe sur Internet?

Dans une affaire récente, GS Media, jugée le 8 septembre 2016 ${ }^{13}$, était posée la question des liens hypertextes. GS Media exploite un site internet très consulté, sur lequel on trouve « des nouveautés, révélations scandaleuses et enquêtes journalistiques sur des sujets amusants et sur un ton de joyeuse plaisanterie ». La plaisanterie n'a pas plu à tout le monde car le site a diffusé des photos destinées à la revue «Playboy » de « Madame Dekker nue ». La société a refusé de les retirer malgré les mises en demeure de l'éditeur de Playboy. La Cour d'appel d'Amsterdam a demandé à la Cour de justice si un site internet qui reçoit un message contenant un lien hypertexte vers un site qui met à disposition une œuvre protégée dont la communication n'a pas été autorisée doit être regardé comme la communiquant au public. Si la réponse est positive il est possible de s'en prendre à lui pour faire cesser la violation des droits et pour réparer le préjudice.

\footnotetext{
10 Le Premier ministre préside la commission supérieure de codification, dont le vice-président est traditionnellement un membre du Conseil d'État, actuellement le président Labetoulle qui a succédé au président Braibant. V. B. StiRn ET Y. AguilA, Droit public français et européen, Dalloz 2014, p. 200 et s. Sur le code des relations entre le public et l'administration : D. LABETOulle, «Le code des relations entre le public et l'administration, Avant-propos », RFDA, 2016-1 et C. BARrois ET M. Vialettes, « La fabrique d'un code ».

$11 \quad$ Aujourd'hui remplacé par le règlement $884 / 2004$

Directive 2001/29.

CJUE, 8 septembre 2016, GS Media, aff. C-160/15
} 
La Cour commence par rappeler que la directive ne donne pas d'autre précision sur la notion de « communication au public », mais elle rappelle que le but est une protection élevée des auteurs et que donc la notion doit être largement conçue. Elle se réfère même à sa jurisprudence sur cette notion pour l'application d'une autre directive, celle sur le droit de location et de prêt dans le domaine de la propriété intellectuelle ${ }^{14}$ car la notion y a la même portée. Elle rappelle les différents critères qu'elle a déjà utilisés et elle précise sa position par rapport à un autre arrêt dans lequel elle avait considéré que le placement de liens hypertextes ne constituait pas une communication au public. En effet, dans cet arrêt le lien renvoyait vers des œuvres librement disponibles sur Internet, ce qui n'était pas le cas des photos en cause ${ }^{15}$. Ensuite, elle examine la question sous l'angle des droits fondamentaux : liberté d'expression et liberté d'entreprise. Cela l'amène à distinguer selon que celui qui place le lien le fait pour des motifs lucratifs ou non : celui qui le fait dans le cadre d'une activité lucrative est présumé savoir que l'œuvre était protégée, présomption qui est réfragable.

Il en résulte que dans le cas d'espèce GS Media doit sans doute être regardé comme ayant communiqué les photos au public au sens de la directive, ce qu'il appartient toutefois à la Cour d'Amsterdam de vérifier.

Dans un autre arrêt récent, la Cour a confirmé sa jurisprudence sur la communication au public dans le cadre d'activités économiques comme les hôtels, restaurants ou établissements thermaux : en mettant à la disposition de ses clients des émissions de télévision, un établissement de traitements postopératoires les communique au public, ce qui a pour conséquence que l'organisme de gestion collective des droits d'auteur peut lui réclamer le paiement de redevances ${ }^{16}$.

Dans un domaine très différent, celui du fameux « doit à l'oubli »sur Internet, l'Avocat général Yves Bot a récemment conclu sur les suites à donner à l'arrêt Google Spain pour ce qui est des sociétés commerciales. Il a proposé à la Cour de justice de dire que ce droit n'existe pas pour les données inscrites au registre des sociétés ${ }^{17}$. La Cour de justice l'a suivi par un arrêt du 9 mars 2017. Certes, il s'agit de données personnelles, mais l'accès prévu par la législation communautaire vise à assurer la sécurité des tiers et cet objectif justifie que ces données soient accessibles même après que la société a cessé ses activités. Ce n'est que dans des circonstances exceptionnelles que des « raisons prépondérantes et légitimes » qu'il faudrait apprécier au cas par cas et compte tenu de temps écoulé depuis la cessation des activités, pourraient venir, sous le contrôle du juge, limiter l'accès des tiers à ces données.

J'ai pris ces deux exemples pour montrer que, quelle que soit la législation, des questions fort importantes, de base même, se posent inévitablement et que cela ne signifie pas qu'elle soit entachée d'un défaut.

\footnotetext{
14 Directive 2006/115, du Parlement européen et du Conseil, du 12 décembre 2006. Dans son arrêt Reha Training, dont il sera question plus loin, la Cour précise que « compte tenu des exigences d'unité et de cohérence de l'ordre juridique de l'Union, les notions utilisées par les directives 2001/29 et 2006/115 doivent avoir la même signification » (pt 28).

CJUE, 13 février 2014, Svensson, aff. C-466/12.

CJUE , 31 mai 2016, Reha Training, aff. C-117/15.

CJUE, 9 mars 2017, Camera di commercio di Lecce, aff. C-398/15.
} 
Le législateur utilise des notions qu'on ne saurait qualifier d'indéterminées car elles ont le degré de précision suffisante et qu'il ne serait même pas raisonnablement possible de les préciser plus avant. La jurisprudence fait ici son travail. Le législateur ne pourrait d'ailleurs pas le faire à sa place, sauf à entrer dans un degré de précision tel qu'il ferait de la législation un dédale et ne ferait d'ailleurs que repousser plus loin les problèmes en faisant apparaitre d'autres questions. La Cour européenne des droits de l'homme résume bien les choses lorsqu'elle dit « ... dans quelque système juridique que ce soit, aussi clair que le libellé d'une disposition légale puisse être, y compris une disposition de droit pénal, il existe inévitablement un élément d'interprétation judiciaire. Il faudra toujours élucider les points douteux et s'adapter aux changements de situation. En outre, la certitude, bien que hautement souhaitable, s'accompagne parfois d'une rigidité excessive ; or le droit doit pouvoir s'adapter aux changements de situation $\gg^{18}$.

\section{2) Venons-en maintenant à un premier type de difficulté que l'on ne rencontre certes} pas que dans le domaine des NTI mais auquel on y est très naturellement plus souvent confronté : la rapidité de l'évolution technologique.

Dans quelle mesure la législation doit-elle être adaptée aux nouvelles réalités ? La jurisprudence peut-elle suffire à faire face ou serait-il préférable de changer les règles et de les mettre au goût du jour?

Deux exemples suffiront à illustrer cet aspect de la question.

Le premier me donne l'occasion de revenir à une affaire dont j'ai parlé au CERIC il y a un an, mais qui n'en était qu'au stade des conclusions : l'affaire New Media Online. Certains se souviennent peut-être qu'était en cause le site internet d'un journal autrichien : le Tiroler Tageszeitung. Le site comprend la version électronique du journal, mais aussi un « onglet » conduisant, lorsque l'on clique dessus, à une page compilant des vidéos d'information locale, sur des sujets politiques, économiques et culturels. Bref, quelque chose de désormais très courant car la presse française fait la même chose, aussi bien les organes nationaux que la presse régionale. Cette page de vidéos est-elle un service de médias audiovisuels « à la demande » au sens de la directive 2010/13 sur les services de médias audiovisuels (SMA) ? La question était posée par le Verwaltungsgerichthof` car si cela avait été le cas le site aurait dû faire l'objet d'une déclaration à l'autorité compétente en matière d'audiovisuel, ce qui n'avait pas été fait. L'Avocat général Szpunar avait proposé de dire que tel n'était pas le cas : cette directive ne viserait pas les portails d'information sur l'Internet. Le doute était permis puisque le $28^{\mathrm{e}}$ considérant de la directive dit qu'elle ne s'applique pas à la version électronique d'un journal. Elle ne concernerait que les sites internet dont l'objectif est de diffuser, à la demande de l'utilisateur, des films ou séries télévisées ou encore des retransmissions d'événements sportifs : en somme, les sites de téléchargement de programmes, qu'ils soient gratuits ou payants. La question était un véritable défi. Ce genre de sujet préoccupe les écosystèmes médiatiques, tant ils sont au cœur des

$18 \quad$ CEDH, 21 octobre 2013, Del Rio Prada c. Espagne, req. 42750/09 (pt 92).

19 Cour administrative suprême d'Autriche. 
bouleversements qu'ont connus, depuis l'entrée en vigueur de la directive 2001/13, les médias audiovisuels et la presse écrite sous l'effet de l'avènement du « tout numérique ».

Quelle est la ratio legis de la directive SMA ? Appliquer, dans un univers médiatique très concurrentiel, les mêmes règles à des acteurs qui s'adressent aux mêmes publics, comme le précise son préambule. Or, il suffit d'observer la manière dont les jeunes générations « consomment » de la vidéo sur Internet pour savoir que la substitution au programme télévisuel linéaire est d'ores et déjà une réalité. Au surplus les gens ne vont pas forcément visionner une vidéo parce qu'ils l'ont trouvée sur un site d'un organe de presse bien particulier : cela se fait de plus en plus indirectement, via un moteur de recherche ou parce que la vidéo a été partagée sur un réseau social. Néanmoins, force est de reconnaître que la directive n'a pas été conçue pour les sites des organes de presse.

La Cour a finalement décidé, contrairement aux conclusions de son Avocat général, qu'un site de ce type entrait dans le champ d'application de la directive ${ }^{20}$. La Cour a justifié sa décision par la nécessité d'appliquer les mêmes règles à des services comparables dans l'univers extrêmement concurrentiel qu'est celui des médias audiovisuels et précisé que de ce point de vue la qualité de l'opérateur - en l'espèce un journal - était indifférente.

On peut se demander s'il faut s'en tenir là ou si le législateur ne devrait pas se ressaisir de la question et réévaluer la situation compte tenu de l'évolution du marché, ce qui d'ailleurs pourrait le conduire à laisser les règles en l'état...

Un second exemple peut être tiré d'une affaire relative à la communication des documents des Institutions : il s'agit de l'affaire Rayner Typke c./ Commission ${ }^{21}$. Elle a donné lieu à des conclusions de l'Avocat Général Bobek prononcées le 21 septembre dernier et à un arrêt du 11 janvier 2017. Il s'agit d'une demande d'accès à des documents sur la base du règlement 1049/2001, du 30 mai 2001, qui est, comme vous le savez, l'équivalent communautaire de notre loi du 17 juillet 1978 sur l'accès aux documents administratifs. L'intéressé voulait un ensemble de données concernant des concours organisés par l'Office européen de sélection du personnel (EPSO). Le requérant a introduit auprès d'EPSO une demande d'accès visant à obtenir, en substance, l'accès à un « tableau » comprenant une série de données rendues anonymes sur les tests en question, en précisant que sa demande ne visait pas à ce qu'EPSO crée en format électronique un nouveau document, mais qu'une version des documents concernés, débarrassés de toute information couverte par l'une des exceptions au droit d'accès, serait suffisante. Cette demande a été rejetée au motif que le document souhaité ne constituait pas un document existant au sens du règlement. Elle viserait, en réalité, la production de nouveaux documents qui ne peuvent pas être extraits d'une base de données relationnelle en effectuant une recherche normale ou de routine à l'aide d'un outil de recherche existant.

Le requérant soutenait que le règlement n'excluait pas de son champ d'application une demande d'accès à une base de données relationnelle, exigeant la formulation de requêtes en langage de

CJUE, 21 octobre 2015, New Media Online GmbH, aff. C-347/14.

aff. C-491/15 P. 
requête structurée, qui n'ont pas été préprogrammées auparavant ou régulièrement utilisées par l'institution. Pour la Commission, en revanche, seuls les résultats des requêtes SQL pouvaient être considérés comme des documents existants.

Selon la définition extensive donnée par le législateur de l'Union, un document peut être tout contenu sur tout support concernant toute activité des institutions de l'Union. La définition de ce qu'est un document dans le cas de bases de données électroniques semble néanmoins exiger davantage de précisions.

Ainsi, se posait la question de savoir si la notion de document peut signifier toute combinaison de données qui pourrait éventuellement être extraite des bases de données grâce à des requêtes complexes.

C'est à ce stade que doit également intervenir la distinction entre « document » et « document existant » dans la mesure où le règlement prévoit seulement l'accès aux documents existants. Or, cette distinction devient particulièrement complexe dans le cas des bases de données.

La principale question qui se pose est la suivante : le document précis en cause existait-il dans une configuration de données spécifique avant que l'on clique, constituant ainsi un document existant, auquel le demandeur a un droit d'accès ou bien s'agit-il de générer un document nouveau auquel le demandeur n'a aucun droit d'accès?

Exclure les documents générés qui n'existaient pas dans une configuration de données spécifique avant le « clic » semble exclure une grande partie des documents qui peuvent être contenus dans une base de données.

Selon l'Avocat général Bobek qui proposait de suivre le raisonnement du Tribunal, il était nécessaire, s'agissant de fichiers, de trouver un autre critère de distinction entre document existant et document nouveau : le degré de caractère dynamique et créatif que présente le processus de génération du document sollicité. Ainsi, seul devrait être regardé comme nouveau un document dont l'obtention nécessite un investissement substantiel qui modifie la base de données elle-même.

Un travail de programmation informatique, comme l'élaboration de nouvelles requêtes SQL et, partant, la création d'un nouveau résultat de recherche dans la base de données, doit être considéré comme nécessitant un investissement substantiel. Le document qu'il génère ne peut donc être considéré comme un document existant.

La Cour a largement suivi son avocat général. Elle relève que pour les bases de données électroniques, la distinction entre un document existant et un document nouveau « doit se faire sur la base d'un critère adapté aux spécificités techniques de ces bases et conforme à l'objectif du règlement $\mathrm{n}^{\circ}$ 1049/2001 » qui est de garantir un accès aussi large que possible aux documents. Elle a, dès lors, jugé que devaient être qualifiées de document existant toutes les informations qui peuvent être extraites d'une base de données électronique dans le cadre de son utilisation courante à l'aide des outils de 
recherche préprogrammés, alors même que ces informations n'auraient pas encore été affichées sous cette forme ou n'auraient jamais fait l'objet d'une recherche par les agents des institutions.

La conséquence en est que, dans le cas des bases de données, les institutions peuvent être conduites à constituer un document à partir des informations qui y sont contenues chaque fois qu'elles peuvent se borner à utiliser les outils de recherche existants. En revanche, serait un document nouveau l'information dont l'extraction d'une base de données nécessiterait un « investissement substantiel », c'est-à-dire une modification, soit de l'organisation de la base de données, soit des outils de recherche actuellement disponibles.

On voit bien ici comment, à partir du même texte qu'est le règlement sur l'accès aux documents, la Cour de justice construit une solution qui permet de passer du cas des documents papier à celui des documents électroniques et de faire ainsi face à l'évolution technologique. Le législateur aurait-il fait mieux? Il est permis d'en douter.

Ces deux exemples, parmi de nombreux autres qu'on pourrait donner, montrent que si l'adaptation de la législation est nécessaire elle doit être soigneusement pesée et que le travail des juges a ce double avantage de permettre au législateur de prendre un peu de temps et de lui donner des éléments de réflexion.

\section{3) Un autre type de difficultés auxquelles je suis assez sensible résulte du caractère très technique de certains textes.}

C’est le cas en matière de télécommunications, de brevets, d'inventions technologiques, de bioéthique...

Ici, la question est simple : de quoi s'agit-il ? De quoi parle-t-on? Qu'a voulu faire le législateur de l'Union? Quelles hypothèses a-t-il voulu viser?

Il faut bien sûr faire la part du feu.

Ainsi, pour définir ce qu'est un «embryon humain », au sens ${ }^{22}$, au moins, de la législation communautaire sur la protection des inventions biotechnologiques, la Cour a-t-elle hésité. Elle a donné une orientation dans son arrêt Brüstle $e^{23}$, puis une autre dans l'arrêt International Stem Cell Corporation après que de nouvelles informations lui eurent été apportées ${ }^{24}$. Le législateur n'a pas autrement défini la notion et on peut se douter pourquoi.

\footnotetext{
Sur le sens de ce « au sens de », fondamental dans la pratique juridictionnelle : C. Vigouroux, «L'expression « au sens de » ou le juge linguiste sans être encyclopédiste », Juger l'administration, administrer la justice, Mélanges en l'honneur de Daniel LABETOULLE, Dalloz 2007, p. 847.

CJUE, 18 octobre 2011, Brüstle/Greenpeace eV, aff. C-345/10.

CJUE, 18 décembre 2014, International Stem Cell Corporation, aff. 364/13.
} 
En revanche, il arrive que le législateur sache exactement de quoi il parle, ou au moins les experts qui ont préparé les textes, mais que les choses soient moins familières aux juges ${ }^{25}$.

J'ai rencontré ce problème notamment dans le domaine des télécommunications.

Un exemple parmi d'autres : l'affaire Base $N V$, un arrêt du 6 octobre $2010^{26}$. Il s'agissait de savoir si la Belgique avait correctement appliqué l'une des directives sur la libéralisation du secteur des télécommunications. La question était la suivante : quand les entreprises chargées du service universel supportent des «coûts injustifiés », ceux-ci peuvent être pris en charge par l'État ou répartis sur les autres entreprises. L'enjeu financier est considérable. Qu'est-ce que des « coûts injustifiés »? La directive dit qu'il faut partir du « coût net ». Mais il est évident que chacun ne le voit pas de la même façon. Peut-être le législateur aurait-il pu donner quelques orientations. À défaut, la Cour est assez largement tributaire des parties et aussi et surtout de la Commission européenne dont on attend toujours beaucoup. Comme vous le savez, la Commission est appelée à donner son avis sur toutes les questions préjudicielles et joue un peu le rôle d'amicus curiae.

Autre exemple avec une affaire récente : CJUE, 17 décembre 2015, Proximus ${ }^{27}$.

Que faut-il entendre par « redevance pour les droits de mettre en place des ressources »?

La Cour était interrogée sur le point de savoir si l'article 13 de la directive « autorisation » s'opposait ou non à ce qu'une taxe soit imposée aux propriétaires de structures, telles que des pylônes et des unités d'émission et de réception, nécessaires au fonctionnement du réseau de télécommunication, comme l'avait fait la Province de Namur, alors que la directive limite les cas dans lesquels peut être imposée une redevance en contrepartie de l'autorisation, afin d'assurer l'utilisation optimale de ressources par définition rares.

Certes, aux termes de la directive, les États restent libres d'imposer des redevances en contrepartie de l'octroi de droits de mettre en place des ressources sur ou sous des biens publics ou privés, mais encore faut-il que ces redevances sur les pylônes question soient considérées comme relevant de cette catégorie.

L'expression «mettre en place des ressources » renvoie aux infrastructures matérielles permettant la fourniture de réseaux et de services et à leur mise en place matérielle sur les propriétés publiques ou privées et tel semblait être le cas dans l'affaire Proximus car les infrastructures étaient utilisées pour des activités couvertes par une autorisation générale accordée sur le fondement de la directive.

La Cour adopte pourtant une solution contraire. Elle considère, en s'en tenant à une lecture formelle des termes utilisés par l'autorité nationale pour désigner la taxe en cause, qu'elle n'est pas

J'ai connu ce genre de difficultés dans le droit de l'urbanisme. Les documents d'urbanisme des années 1970, après la loi d'orientation foncière de 1967, étaient largement rédigés par des ingénieurs, au demeurant fort qualifiés, mais qui n'étaient pas de juristes. Ils se comprenaient très bien entre eux, mais la portée des dispositions des plans d'occupation des sols de l'époque ont laissé plus d'un juge perplexe. Fort heureusement le mode de rédaction des documents a changé et les juristes participent de plus près à leur établissement.

CJUE, 6 octobre 2010 Base NV, aff. C-389/08.

CJUE, 17 décembre 2015, Proximus, aff. C-517/13 
liée à la procédure d'autorisation dès lors qu'elle s'applique à toute personne qui exploite des installations situées sur le territoire de la province.

Or, un examen de la réalité concrète aurait pu montrer que seules y sont assujettis les exploitants de réseaux et de communications de téléphonie mobile, titulaires d'autorisations d'exploitation et souhaitant mettre en place des ressources dans le cadre de leurs activités, ces infrastructures étant par ailleurs absolument nécessaires à la poursuite des activités des opérateurs. Dans ce cas, il s'agirait d'une imposition additionnelle, à qualifier de redevance au sens de l'article 13, et légale seulement si elle en remplissait les conditions.

Cet arrêt ne met pourtant pas fin aux incertitudes qui entourent les taxes sur les pylônes, dans la mesure où la Cour considère qu'il appartient à la juridiction nationale de vérifier si, en dépit de la formulation utilisée par le législateur national, celle-ci ne vise pas en réalité à instaurer une charge additionnelle pour l'opérateur de téléphonie mobile titulaire d'autorisation d'exploitation ${ }^{28}$.

Le législateur de l'Union pourrait opportunément, à l'occasion du processus de révision du cadre règlementaire des communications électroniques, préciser les cas dans lesquels peuvent être prévues de telles taxes.

\section{4) J'en viens maintenant au rôle que le juge peut jouer dans l'amélioration de la législation européenne.}

Je me limiterai à trois séries de rapides remarques.

a) Par sa jurisprudence constante le juge peut conduire à un remodelage des textes. Comme je l'ai dit plus haut, le législateur de l'Union prend en compte la jurisprudence afin d'actualiser les règlements et directives par la voie d'une codification, au sens communautaire du terme.

b) Le juge contribue aussi à éclairer la législation. Cela le conduit même à la compléter. Il doit avoir le souci de lever les ambiguïtés par une formulation de ses arrêts aussi compréhensible que possible. Il s'agit là d'une « ardente obligation ».

c) Il me semble enfin que la Cour de justice pourrait aussi appeler l'attention du législateur sur les insuffisances de la législation qui se sont révélées au fil des affaires dans le cours d'une année judiciaire. Cela pourrait faire l'objet d'une partie de son rapport annuel. Bien évidemment, il ne s'agirait pas de prendre parti sur les orientations de fond qui relèvent du seul pouvoir politique, mais plus modestement de pointer telle obscurité ou telle insuffisance, souvent d'ordre technique. Cela contribue grandement à la prévention du contentieux qui devient, dans le système communautaire, aussi nécessaire que dans les États membres. Je ne méconnais pas que les traditions sont différentes, mais je pense que participer ainsi, au fur et à mesure, à l'amélioration du corpus législatif, fait partie de la mission des juges.

CJUE, 8 juillet 2015, Ville de Mons, aff. C-346/16, pt 23. 



\title{
LES PRINCIPES DU « MIEUX LÉGIFÉRER 》 DANS LE DOMAINE DE L'ENVIRONNEMENT ET DE LA SANTÉ Quel bilan? Quelles PERSPectives?
}

\author{
Estelle BrossET ${ }^{1}$
}

\section{Résumé}

Si les contours conceptuels du «Mieux légiférer »sont souvent décryptés, ses conséquences pratiques le sont moins souvent alors même que d'une telle analyse est essentielle, voire plus importante que la première. Y répondre exige de dresser le bilan de l'application des principes du « Mieux légiférer » dans les différents pans du droit de l'Union. Dans cette contribution, il s'agira de dresser le bilan dans les domaines du droit de l'environnement et de la santé. À l'analyse, la conclusion ne fait pas de doute : la mise en application desdits principes a été et est importante dans ces matières ce qui peut aisément s'expliquer par la correspondance forte entre les exigences du Mieux légiférer et un grand nombre des principes structurants de ces domaines. Logiquement, eu égard à cette généreuse application, des débats, parfois cruciaux, à propos du programme « Mieux légiférer », de ses outils comme de ses logiques ont été, dans ces deux domaines, révélés et un certain nombre de réponses éprouvées. Les domaines de l'environnement et de la santé font donc tout à la fois apparaître une mise en application (1) et une mise en tension (2) des principes du « Mieux légiférer ».

\section{Abstract}

If the abstract outlines of the "Better regulation Initiative" are often analysed, its practical consequences are it less often even though such an analysis is essential, even more important than the first one. To answer, it require to evaluate the implementation of the "Better regulation" principles in the various pieces of the European Union Law. In this contribution, an overview of the implementation in the environmental and health fields will be given. The conclusion is the following one: the implementation in this fields was and will be important what can be easily explained by a high degree of compatibility between this principles and the structural principles in these fields. Logically, number of questions, sometimes crucial, about the "Better regulation Initiative", its tools, its rationality, were, in the environmental and health fields, revealed and some answers given. In brief, in these fields, an application (1) and, at the same time, a "tensionning" (2) of the "Better regulation" principles can be observed.

1 Professeure de droit public, Chaire Jean Monnet, Aix Marseille Univ, Université de Toulon, Univ Pau \& Pays Adour, CNRS, DICE, CERIC, Aix-en-Provence, France. 
« Promis, juré, on ne l'y reprendra plus. La main sur le cœur, la Commission européenne assure qu'elle a rompu avec ses vieux démons, letrop-pleinderéglementations. Finiletempsoùelledécidait de la taille et du poids que devaient avoir toutes les cucurbitacées d'Europe, elle veut désormais alléger le fardeau administratif. Qu'on ne compte plus sur elle pour tendre le concombre pour se faire battre ! Le maître mot de sa nouvelle approche [...] : « rendre la législation européenne plus légère, plus simple et moins chère ${ }^{2}$.»

«En parallèle de sujets prééminents sur les questions économiques, migratoires ou internationales, d'autres initiatives publiques liées à la politique européenne quotidienne et son processus décisionnel ordinaire [...] ont été enclenchées de manière relativement inaperçue $»^{3}$. C'est le cas de l'initiative en faveur du « Mieux légiférer » et dès lors, on ne peut que se féliciter que des ouvrages lui sont consacrés ${ }^{4}$. Pourtant, une telle initiative est loin d'être nouvelle. Certes, il y a peu, à l'occasion du discours du président Juncker sur l'état de l'Union en 2016, la Commission présentait les progrès déjà réalisés et les mesures à prendre ${ }^{5}$ dans le cadre de l'application du programme «Améliorer la réglementation » présenté un an plus tôt ${ }^{6}$. Et quelques mois plus tôt, était adopté, le 13 avril 2016, entre le Parlement, le Commission et le Conseil, l'accord interinstitutionnel précisément intitulé « Mieux légiférer » ${ }^{7}$. Toutefois, depuis plus de dix années, le « Mieux légiférer » est clairement un objectif politique affiché dans l'Union, particulièrement, mais pas seulement, du côté de la Commission européenne. Pour s'en convaincre, il suffit de citer le Livre blanc sur la gouvernance de $2001^{8}$, la Communication de la Commission sur la « Gouvernance européenne : Mieux légiférer $»^{9}$ ou encore l'accord institutionnel « Mieux légiférer » de 2003 entre le Parlement, le Conseil et la Commission ${ }^{10}$.

Reconnaissons-le, in abstracto, l'initiative a de quoi intriguer, l'adjectif « mieux » étant pour le moins minimaliste, indiquant simplement que la réglementation doit être meilleure, plus convenable. La formule devait donc être définie. Parmi plusieurs définitions, dans le dernier accord interinstitutionnel, les trois institutions conviennent, pour mieux légiférer, « de promouvoir la simplicité, la clarté et la cohérence dans la rédaction de la législation de l'Union, ainsi que la plus grande transparence du processus législatif [...] (et) prévoir des exigences appropriées en matière d'information, de suivi et d'évaluation, (et) d'éviter la réglementation excessive et les lourdeurs administratives ». Les objectifs

\footnotetext{
R. HonORÉ, « L'Europe et le complexe du concombre », Les Echos, 03.10.2003.

C. De Marcilly, M. Touillon, « Programme "Mieux légiférer" : l'expertise surpasse-t-elle le politique ? "Question d’Europe n 370,

Comme celui-ci mais voir également : F. Peraldi-Leneuf, S. De la Rosa (éd.), L’Union européenne et l'idéal de meilleure législation, Cahiers européens, Pedone, 2013, 246 p.

5 Communication de la Commission au Parlement européen, au Conseil européen et au Conseil, Améliorer la réglementation: de meilleurs résultats pour une Union plus forte, COM (2016) 0615 final.

6 Communication de la Commission au Parlement européen et au Conseil, Améliorer la réglementation pour obtenir de meilleurs résultats Un enjeu prioritaire pour l'UE, COM (2015) 215 final.

7 Accord interinstitutionnel entre le Parlement européen, le Conseil de l'Union européenne et la Commission européenne « Mieux légiférer », fO L 123 du 12.05.2016, p. 1-14.

COM (2001) 428 final.

COM (2002) 275 final/2.

10 Accord interinstitutionnel, « Mieux légiférer », $\mathcal{F O ~ n}{ }^{\circ} \mathrm{C} 321$ du 31.12.2003, p. 1-5.
} 9.11.2015. 
sont finalement assez classiques, dépassant de loin l'Union et son droit ${ }^{11}:$ il s'agit en effet «tout à la fois de réduire le poids des réglementations - réglementer moins (smart regulation) - et d'améliorer leur contenu - réglementer mieux (better regulation) $»^{12}$. Le $"$ mieux $»$ renvoie clairement à ces deux aspects. On doit d'ailleurs toutefois admettre que le premier aspect a été, depuis quelques années, nettement valorisé dans les documents de la Commission en le reliant à un objectif particulier, celui de la croissance et des emplois ${ }^{13}$. «Le programme d'action sur la réduction des charges administratives, lancé en 2007 par la Commission Barroso I, a initié une forme de rupture [...] dans la mesure où il établit un lien de causalité entre charge réglementaire et compétitivité. L'objectif de qualité cédait alors sa place à un objectif utilitariste de la réglementation : aider les entreprises à se positionner dans la compétitivité internationale en diminuant les coûts de conformité $»^{14}$. En 2012, le lancement du programme REFIT ${ }^{15}$ pour une réglementation affûtée et performante ${ }^{16}$ confirme la nouvelle configuration puisque le programme vise de manière affichée à supprimer les coûts inutiles générés par la réglementation (c'est-à-dire les charges). Le Mieux légiférer se confond donc de plus en plus souvent à du «moins » légiférer avec toutes les critiques que ce glissement peut susciter tant du point de vue de son principe que de son résultat. En effet, il n'est pas anodin, au plan du principe, de considérer la réglementation comme une charge et en tous les cas, cette assimilation mérite d'être discutée. Surtout, du point de vue de ses résultats, cette assimilation ne produit pas mécaniquement l'effet escompté puisque « tout passage d'un état $\mathrm{A}$ du cadre juridique à un état B présentant moins de complexité et de textes nécessite de nouveaux textes et arbitrages et donc produit finalement un volume supérieur de textes $»^{17}$.

Quoique discutables, les contours conceptuels du « Mieux légiférer » apparaissent donc avec une relative netteté. Tel n'est pas le cas des conséquences pratiques, empiriques alors même que cette question est tout autant, voire sans doute plus, importante que la première. Y répondre exige de dresser le bilan de l'application du programme dans les différents pans du droit de l'Union ce qui est ambitieux. Dans cette contribution, il s'agira de dresser le bilan dans les domaines du droit de l'environnement et de la santé. A priori, de tels domaines pouvaient pourtant paraître « contre-intuitifs » pour une réflexion sur le « Mieux légiférer ». Il faut dire que, dans une vue générale, l'émergence d'une prise en compte par les pouvoirs publics d'une préoccupation en termes de rationalisation de l'action publique, d'une part, et de prise en compte de la protection de l'environnement et de la santé d'autre part, se fait à peu près à la même période ${ }^{18}$ à tel point que « moderniser un

11 M. Fatin-Rouge Stefanini, L. Gay, J. Pini (éd.), Autour de la qualité des normes, Bruylant, coll. À la croisée des droits, 2010,328 p.

12 On les retrouve, sous des formes diverses, dans la plupart des droits nationaux : J. CHEvallier, « La simplification de l'action publique et la question du droit », Revue française d'administration publique, 1/2016 ( $\left.{ }^{\circ} 157\right)$, p. 205-214.

13 Communication de la Commission au Conseil et au Parlement européen, Améliorer la réglementation en matière de croissance et d'emploi dans l'Union européenne, COM/2005/0097 final.

14 E. VAn DEN AbeELE, « The better regulation agenda : a 'new deal' in the building of Europe? European social policy », Policy Brief, 1/2009, Bruxelles, ETUI.

15 Communication de la Commission, Pour une réglementation de l'UE bien affûtée, COM/2012/0746 final.

16 «REFT» pour « Regulatory Fitness and Performance Programme».

17 B. Du MARAIs, «Simplifier le droit : du mythe de Sisyphe à l'horticulture juridique ? », Revue française d'administration publique, $1 / 2016$ $\left(\mathrm{N}^{\circ} 157\right)$, p. $183-204$.

18 C. BLATRIX, « Moderniser un droit moderne ? Origines et significations de la simplification de l'action publique environnementale », Revue française d'administration publique, 1/2016 ( $\left.{ }^{\circ} 157\right)$, p. 67-82. 
droit moderne $»^{19}$ pouvait paraître quelque peu incongru. Toutefois, en regardant plus finement, on constate sans difficulté que la modernité des droits de l'environnement et de la santé, notamment (mais pas seulement) de l'Union européenne, doit être sérieusement relativisée. C'est en 1985 puis 1992 qu'ont été attribuées à l'Union des compétence en ces matières. Or, 1985, 1992, ce n'est plus hier ! Et surtout, bien avant ces dates, existait dans l'Union européenne un acquis notable en ces matières rendu possible par le recours important à d'autres compétences, particulièrement la compétence en matière de marché intérieur (article 114 TFUE) ${ }^{20}$. Il n'est dès lors pas surprenant de repérer également des exemples d'application du programme « Mieux légiférer » en ces matières, l'exemple le plus médiatisé ayant été l'annonce par la Commission dans le cadre de son programme de travail pour l'année $2015^{21}$ de son intention de retirer de son programme de travail les paquets « air pur » et « déchets » (soit l'instrument législatif du paquet « économie circulaire »). Cette proposition de retrait a été rapidement et vivement critiquée ${ }^{22}$. Et pourtant ${ }^{23}$, le paquet sur l'économie circulaire a été tout de même retiré par la Commission, sauf quelques éléments ${ }^{24}$. Or, la Commission justifia un tel retrait précisément par l'approche « Mieux légiférer », soulignant qu'un tel retrait « réduit la bureaucratie et supprime des charges réglementaires, contribuant de la sorte à la mise en place d'un environnement propice aux investissements », tout en promettant une approche plus « ciblée » qui la conduirait à faire de nouvelles propositions ${ }^{25}$. Certes, le pouvoir d'initiative revient à la Commission et de ce fait, il est logique qu'elle dispose également du droit de retirer sa proposition ce que la Cour a elle-même confirmé. Il n'en demeure pas moins, qu'un tel retrait a marqué les esprits. Dans ce contexte, que les critiques ${ }^{26}$ et la vigilance ${ }^{27}$ à l'endroit du Mieux légiférer soient, dans ces matières, devenues fortes n'étonne guère.

Il convenait toutefois, au-delà de cet exemple fortement médiatisé, d'examiner, dans une perspective d'ensemble, si et comment le « Mieux légiférer » a été mis en ouvre dans ces domaines. À l'examen, la conclusion ne fait pas de doute : la mise en application a été et est importante dans ces matières ce qui, de notre point de vue, peut aisément s'expliquer par la correspondance forte entre

19 C. BLATRIX, « Moderniser un droit moderne ? Origines et significations de la simplification de l'action publique environnementale », ibidem. 20 V. MICHEL, «Les objectifs à caractère transversal », in E. NEFRAMI (éd.), Objectifs et compétences dans l'Union européenne, Bruxelles, Bruylant, 2013, p. 113.

21 Voir l'annexe de la Communication de la Commission, Programme de travail pour l'année 2015, COM (2014) 910 final.

22 Lors du Conseil (Environnement) du 17 décembre 2014, les délégations nationales se sont majoritairement opposées au retrait. Du côté du Parlement européen, l'ensemble des groupes politiques a été également très critique par rapport au retrait. Le Parlement européen n’a pas cependant réussi à rassembler la majorité nécessaire en séance plénière pour adopter une résolution sur l'ensemble des intentions annoncées par la Commission pour son programme de travail. Cependant, le président de la commission ENVI, Giovanni LAVIA (PPE - IT) a envoyé une lettre très claire au vice-Président Timmermans le 18 décembre 2014.

23 E. VAN Den ABeele, « Mieux légiférer» : une simplification bureaucratique à visée politique, Working Paper, 2015.04, p. 52-53.

24 Pour ce qui concerne le paquet « air pur », il dut toutefois renoncer à retirer la directive sur les plafonds d'émissions nationaux (NEC), la Commission annonçant qu'elle serait susceptible de l'amender à un stade ultérieur. Il dut également renoncer à retirer la proposition de directive sur les émissions des installations de combustion de taille moyenne.

25 Il faut signaler que, comme annoncé par la Commission au moment du retrait, un plan d'action et des nouvelles propositions législatives en faveur d'une économie circulaire ont été publiés le 2 décembre 2015 : Voir la Communication de la Commission au Parlement européen, au Conseil, au Comité économique et social européen et au Comité des régions du 2 décembre 2015 : Boucler la boucle - Un plan d'action de l'Union européenne en faveur de l'économie circulaire (COM (2015) 614 final).

26 Les rapports, études et prises de position des parties intéressées sont nombreux et leur titre évocateur : voir pour exemple le rapport de l'Institut pour la politique européenne environnementale : « For Better or for Worse ? The EU's 'Better Regulation' Agenda and the Environment » disponible à l'adresse : http://www.ieeplondon.org.uk/assets/476/FinalReport_pdf10.pdf.

27 On pense à la création, en réponse à la présentation par la Commission de son programme, par les organisations de la société civile d'une plateforme de surveillance nommée « Better regulation Watchdog » visant à s'assurer que l'agenda ne serait pas mis au service d'un affaiblissement des législations européennes notamment en matière de droit des consommateurs et des citoyens ainsi qu'en matière d'environnement : http://www.betterregwatch.eu/. 
les exigences du Mieux légiférer et un grand nombre des principes structurants de ces domaines. Logiquement, eu égard à cette généreuse application, des débats, parfois cruciaux, à propos du programme « Mieux légiférer », de ses outils comme de ses logiques ont été révélés, parfois même ces secteurs ont permis de fournir quelques réponses. Les domaines de l'environnement et de la santé font donc tout à la fois apparaître une mise en application (1) et une mise en tension (2) des principes du Mieux légiférer.

\section{1) La mise en application des principes du «Mieux légiférer»}

Il n'est pas utile, eu égard aux déjà nombreuses contributions sur ce thème, de revenir sur ce qu'implique précisément le « Mieux légiférer », simplement rappeler qu'il est, en règle générale, associé à au moins quatre grands principes : la réduction des charges réglementaires et administratives, l'évaluation de l'impact ex-ante, l'évaluation ex-post d'une législation et la consultation des parties prenantes. Or pour ces quatre grands principes, la mise en application est tout à fait importante et à cet égard, les domaines de l'environnement et de la santé fournissent des exemples emblématiques (1.1). Cela n'est guère surprenant. En effet, les principes du «Mieux légiférer» croisent souvent les principes structurants des droits de l'environnement et de la santé au point que la mise en application était en quelque sorte attendue, naturelle (1.2).

\subsection{Une importante mise en application}

Suivant les principes du « Mieux légiférer » cités juste avant, il est possible de trouver, pour chacun, de nombreux exemples d'application dans le domaine de l'environnement et de la santé. Pour des raisons bien compréhensibles dans le cadre d'une simple contribution à un ouvrage, nous n'en citerons que quelques uns.

S'agissant du premier principe, de réduction des charges règlementaires et administratives, les exemples sont en effet tout à fait nombreux car plusieurs révisions de textes de l'Union en matière de santé ou d'environnement ont été animées, entre autres, par un tel objectif. Pour ne prendre qu'un exemple, l'adoption en 2006 du règlement REACH relatif à l'enregistrement, l'évaluation et l'autorisation des substances chimiques ${ }^{28}$ est tout à fait évocateur. L'adoption d'un tel règlement avait en effet pour objectif de rassembler en un texte, sous la forme d'un règlement, un vaste corpus règlementaire (près de 40 textes) auparavant quasiment exclusivement constitué de directives ${ }^{29}$. Il avait également pour objectif d'en modifier, de manière assez radicale, les obligations principales avec pour souci premier de ne pas faire peser sur l'industrie chimique de charges réglementaires excessives, notamment en substituant au dispositif précédent d'autorisation de mise sur le marché

28 Règlement (CE) n ${ }^{\circ}$ 1907/2006 du Parlement européen et du Conseil du 18 décembre 2006 concernant l'enregistrement, l'évaluation et l'autorisation des substances chimiques, ainsi que les restrictions applicables à ces substances (REACH) et instituant une agence européenne des produits chimiques, $\mathrm{FO} \mathrm{n}^{\circ} \mathrm{L} 396,30.12 .2006$, p. 1-850.

29 D'ailleurs, l'une des toutes premières réglementations en matière de protection de l'environnement, alors même que les traités ne reconnaissaient aucune compétence à la Communauté dans ce domaine, couvre cette matière et prenait la forme d'une directive : Directive 67/ 548/CEE, 27 juin 1967 concernant le rapprochement des dispositions législatives, réglementaires et administratives relatives à la classification, l'emballage et l'étiquetage des substances dangereuses (fO 196 du 16.08.1967, p. 1-98). 
un système d'enregistrement (et donc de communication des données par l'industriel) gradué en fonction des quantités produites ${ }^{30}$. On notera d'ailleurs que ce règlement continue à fournir une illustration de l'application du « Mieux légiférer». En effet, dans son programme pour $2016^{31}$, la Commission a fait apparaître parmi ses initiatives dites REFIT, une initiative relative au règlement REACH qui vise l'adoption d'un règlement d'exécution de la Commission relatif à la simplification de la procédure d'autorisation prévue par exception dans le cadre dudit règlement... ${ }^{32}$.

Le principe de l'évaluation ex-ante et ex-post qui fait partie du noyau dur du "Mieux légiférer » connaît également des applications nombreuses dans les domaines de l'environnement et de la santé. D'abord, le domaine de l'environnement et de la santé fournit quelques exemples pour le moins précurseurs tout autant qu'archétypales de la pratique de l'analyse d'impact, instrument central de l'évaluation ex ante. Pour reprendre l'exemple précédent, cela a été par exemple le cas des analyses d'impact réalisées préalablement à l'adoption du règlement $\mathrm{REACH}^{33}$. Et pour cause, outre l'analyse d'impact - déjà très détaillée - de la Commission, le secteur industriel a obtenu, de façon tout à fait exceptionnelle, le feu vert de la Commission pour la réalisation de deux études d'impact supplémentaires afin de pronostiquer non seulement les coûts mais aussi les gains attendus par cette nouvelle réglementation. En outre, de telles analyses ont clairement joué un rôle clef dans l'adoption du règlement ${ }^{34}$. S'agissant du principe de l'évaluation ex-post, les domaines de l'environnement et de la santé offrent également plusieurs exemples. Et pour cause, un grand nombre de bilans de qualité - outils de l'évaluation ex post - ont été lancés dans les domaines de l'environnement et de la santé. Le fitness check des directives Habitats et oiseaux a ainsi été particulièrement remarqué tant les craintes ont été nombreuses à son endroit ${ }^{35}$. Toutefois, le rapport d'évaluation rendu en mars $2016^{36}$ conclut à l'efficacité des directives non seulement du point de vue de leur mise en œuvre juridique, mais également du point de vue de l'efficacité réelle de ces dernières sur la protection des oiseaux sauvages et des habitats. En effet, $52 \%$ des espèces d'oiseaux sont en sécurité pour $17 \%$ menacées et

30 L'enregistrement n'est en effet obligatoire que si la substance est présente dans les produits dans des quantités supérieures au total à 1 tonne par producteur ou importateur par an. Le volume détermine en outre les obligations afférentes à l'enregistrement pour l'industriel puisqu'entre 1 et 10 tonnes par an et par producteur et importateur, elles sont singulièrement allégées et le délai pour s'y conformer est allongé. Au-delà de cette condition de volume, il est nécessaire que la substance réponde aux critères de classification des substances dangereuses et qu'elle soit destinée à être rejetée dans des conditions normales et raisonnablement prévisibles d'utilisation. Si la libération d'une substance dangereuse n'est pas intentionnelle et si la quantité rejetée peut avoir des effets nocifs sur la santé, un dispositif minimal est prévu : les substances doivent être notifiées et, à la suite de la notification, il est possible de décider de soumettre celles-ci aux obligations du règlement. 31 Communication de la Commission au Parlement européen et au Conseil, Programme de travail de la Commission pour 2016 : L'heure n'est plus à une gestion conventionnelle, COM/2015/0610 final.

32 Rappelons qu'une procédure d'autorisation est prévue pour certaines substances considérées comme des substances extrêmement préoccupantes même celles non enregistrées, notamment les substances produites en quantité inférieure à une tonne par an. Il s'agit d'abord des substances cancérigènes, mutagènes et toxiques pour la reproduction (CMR) de catégorie 1 (toxicité avérée) et 2 (forte présomption de toxicité) et des substances persistantes, bio-cumulatives et toxiques (dites PBT ou vPvB). Or, le dossier de demande d'autorisation est substantiel puisque la procédure est prévue pour des substances très risquées. Les coûts afférents sont donc importants. La simplification programmée vise à les diminuer en réduisant les obligations relatives notamment aux demandes d'autorisation pour la production de substances en faibles quantités.

33 P. Schuseil, Les études d'impact du règlement Reach, Direction général du Trésor, 2013, disponible à l'adresse : http://www.tresor.economie. gouv.fr/File/396955.

34 Pour une étude précise : voir P. Schuseil, Les études d'impact du règlement Reach, ibidem.

35 Il faut dire que ce qui est demandé suscite des interrogations. Dans la lettre de mission de J-C. Juncker au Commissaire de l'environnement Karmenu VelLA il est dit : "I would like you to focus on...continuing to overhaul the existing environmental legislative framework...and assess the potential for merging them [the Birds and Habitats Directives] into a more modern piece of legislation ». "Le spectre d'un assouplissement des directives sous couvert de leur "modernisation » a suscité une forte mobilisation tant des ONG que du public en faveur d'un maintien des directives - pas moins de 520000 citoyens ayant participé à la consultation organisée par la Commission dans le cadre de cette procédure »: C-H. BorN, « Chronique de droit européen de la biodiversité - 2014-2015 », Revue juridique de l'environnement, 4/2016 (Volume 41), p. 745-786. 36 Evaluation Study to support the Fitness Check of the Birds and Habitats Directives, Final Report, March 2016. 
$15 \%$ en régression ou appauvries ${ }^{37}$. En outre, le rapport conclut que les coûts (5,8 milliards d'euros par an à travers l'Union) des directives sont raisonnables au regard des objectifs poursuivis et des résultats obtenus (bénéfices ${ }^{38}$ ). Au final, sur cette base, aucune suggestion de changement majeur n'est évoquée dans le rapport.

S'agissant enfin de la consultation des parties prenantes en vue du « Mieux légiférer », là encore les domaines de l'environnement et de la santé offrent des exemples nombreux. Et pour cause, le nombre de consultations du public y est substantiel : par exemple, dans le domaine de l'environnement, il est possible d'en dénombrer 168 closes entre 2000 et 2016 et 4 sont en cours. De plus, dans ce domaine, les consultations donnent souvent lieu à de nombreuses contributions. Un nombre record de 500000 personnes a ainsi formulé des observations dans le cadre de la révision des directives «Oiseaux» et «Habitats» dont on vient de parler. Notons également que, dans certains cas, la consultation a joué un rôle déterminant dans la formation de la règle. L'exemple du règlement $\mathrm{REACH}$ peut être ici à nouveau utilisé. À la suite de son Livre blanc sur le sujet, la Commission avait en effet organisé une consultation par internet sur un avant-projet de règlement. La participation à la consultation a été importante puisque 6000 contributions ont été recueillies, en provenance de gouvernements européens, de partenaires commerciaux de l'Union, de l'industrie, des ONG. Or, les principales préoccupations exprimées dans ces contributions concernaient en partie les coûts du système d'enregistrement des substances chimiques ${ }^{39}$. Suite à ces réactions, la Commission a adopté en 2003 une nouvelle proposition fortement amendée avec pour objectif une diminution des coûts (notamment par la modulation des obligations en fonction d'un tonnage révisé). Depuis l'adoption du règlement $\mathrm{REACH}$, les parties prenantes continuent d'ailleurs à participer à sa mise en application ${ }^{40}$.

\subsection{Une naturelle mise en application}

L'application importante des principes du « Mieux légiférer » dans ces domaines peut s'expliquer sans grande difficulté. Elle est reliée au fait que de tels principes, particulièrement celui de l'évaluation et celui de la consultation, se combinent idéalement avec les principes propres au domaine de l'environnement et de la santé.

\footnotetext{
37 Les espèces d'oiseaux communs présents en zone rurale, plus particulièrement dans les zones agricoles, sont les plus susceptibles de voir leur population décliner. Seuls $16 \%$ des habitats montrent un statut de conservation favorable, tandis que la plupart des autres un statut seulement défavorable, inadéquat (47\%) ou défavorable, médiocre (30\%) : Evaluation Study to support the Fitness Check of the Birds and Habitats Directives, Final Report, March 2016, p. 23.

38 La protection et l'amélioration des statuts des habitats et des espèces constituent les bénéfices essentiels de la mise en œuvre des directives mais ils contribuent également à l'amélioration des prestations des services écosystémiques, estimées à 200 à 300 milliards d'euros par an ainsi qu'à des bénéfices aux économies locales, par la création d'emplois et le tourisme.

39 J-N. Jouzel, P. LAscoumes, « Le règlement REACH, une politique européenne de l'incertain », Politiques européennes, 2011, 33 (1), p.185-214.

40 Elles ont un rôle au sein de l'ECHA Management Board (Le conseil d'administration) composé comme suit : 28 membres des États membres de l'UE ; 3 représentants de la Commission ; 2 représentants du Parlement européen et observateurs représentant les parties intéressées. Elles ont également un rôle via les comités techniques (comité d'analyse socio-économique ou le comité d'évaluation des risques). Les parties prenantes apportent également leur contribution à l'évaluation socio-économique grâce aux périodes de commentaires. Elles sont également impliquées dans la révision régulière des annexes de $\mathrm{REACH}$. En effet, par exemple, une procédure de consultation est prévue pour les propositions réglementaires de restriction d'utilisation.
} 
Le premier exemple concerne l'évaluation ex ante et le mécanisme de l'analyse d'impact qui coïncident parfaitement avec un principe d'importance pour ces domaines, le principe d'intégration des considérations environnementales et sanitaires dans toutes les politiques de l'Union. Ce principe fait en effet partie des principes premiers d'exercice de la compétence dans le domaine de l'environnement et de la santét ${ }^{41}$. Il ne s'agit pas simplement d' "une déclaration d'intention, (d')un engagement politique destiné à assurer que le développement d'une politique sectorielle ne se fera pas au détriment des principes directeurs d'une autre politique sectorielle $»^{42}$. C'est une obligation juridique de tenir compte des exigences sanitaires et environnementales dans toutes les politiques et actions. «Finalement, ladite politique sectorielle devient, par la clause d'intégration, un objectif de l'Union auquel le législateur européen doit avoir égard en plus des objectifs généraux énoncés à l'article 3 TUE $»^{43}$. Or, aux côtés d'autres instruments ${ }^{44}$, l'analyse de l'impact des initiatives législatives de la Commission prises en dehors des domaines de l'environnement et de la santé permet clairement de concrétiser ledit principe. En effet, parmi les impacts envisagés, les incidences positives et négatives associées à l'évolution de l'environnement (telles que l'évolution climatique, la pollution de l'air, de l'eau et du sol, le changement d'affectation des sols, la perte de biodiversité...) sont partie de l'analyse d'impact ${ }^{45}$. Parmi les incidences sociales, les effets sur la santé et la sécurité des individus/populations, y compris sur leur espérance de vie et leur mortalité sont aussi mesurés. Il faut d'ailleurs relever que les impacts sur la santé et l'environnement ont été évalués de manière de plus en plus fréquente, même si l'augmentation ${ }^{46}$ a été très progressive ${ }^{47}$. Par exemple, en 2005, un peu plus de $30 \%$ des études d'impact avaient évalué l'incidence environnementale. En 2011, les conséquences environnementales ont été évaluées dans presque $70 \%$ des études d'impact ${ }^{48}$. Ajoutons que le principe de l'évaluation ex post dans le cadre du « Mieux légiférer » s'imbrique aussi à un principe ancien et important en matière d'environnement, celui du suivi de l'application des obligations en matière d'environnement. Il faut dire que parmi les dossiers qui ont donné lieu à des procédures formelles d'infraction, l'environnement arrive depuis longtemps en tête ${ }^{49}$. Il n'est dès lors pas étonnant que l'obligation de suivi de l'application des textes adoptés dans ce domaine soit particulièrement développée et depuis longtemps. En 1990, le Conseil européen de Dublin invitait déjà la Commission à utiliser le système des rapports périodiques en vue d'assurer une mise en œuvre effective de l'acquis communautaire en matière de

$41 \quad$ Voir l'article 11 TFUE selon lequel «Les exigences de la protection de l'environnement doivent être intégrées dans la définition et la mise en ouvre des politiques et actions de l'Union». Voir également l'article 37 de la Charte des Droits fondamentaux de l'Union européenne. Il est énoncé dans une forme très similaire en matière de santé à l'article 168 § 1 TFUE, à l'article 35 de la Charte et à l'article 9 TFUE : «Dans la définition et la mise en oeuvre de ses politiques et actions, l'Union prend en compte les exigences liées à [...] la protection de la santé humaine »

42 V. Michel, «Les objectifs à caractère transversal », in E. Neframi (éd.), Objectifs et compétences dans l'Union européenne, Bruxelles, Bruylant, 2013, p. 175.

43 Ibidem.

44 Voir les exemples donnés par S. GuIGNER, La santé : un enjeu vital pour l'Europe, Notre Europe, Études et recherches n 68, 2009, 52 p.

45 Commission européenne, Lignes directrices concernant l'analyse d'impact, 15.01.2009, SEC (2009) $92 \mathrm{p}$.

46 A. RENDA, «Les études d'impact des réglementations de l'Union européenne : état des lieux et pistes de réforme », Revue française d'administration publique 1/2014 ( $\left.\mathrm{N}^{\circ} 149\right)$, pp. 79-103

47 C'est ainsi le cas de l'évaluation de l'impact sur la santé : un examen des analyses d'impact réalisées par la Commission pendant deux années (2005-2006) a permis de conclure que plus de la moitié ne mentionnait même pas le mot « santé ». T. STAHL, Is Health Recognised in the EU's Policy Process? An analysis of the European Commission's impact assessments. European Journal of Public Health, Advance Access Published Online 23 June 2009. https://academic.oup.com/eurpub/article/20/2/176/533636/Is-health-recognized-in-the-EU-s-policy-process-An 48 A. RENDA, «Les études d'impact des réglementations de l'Union européenne : état des lieux et pistes de réforme », op.cit.

49 C'est encore le cas en 2015 avec 276 procédures d'infraction lancées : Rapport de la Commission, Le contrôle de l'application du droit de l'Union européenne, Rapport annuel 2015, le 15.07.2016, COM(2016) 463 final. 
protection de l'environnement ${ }^{50}$. Depuis, la plupart des directives prévoient l'obligation pour les États membres de rendre un rapport sur l'application du texte. La directive « Habitats ${ }^{51}$ organise par exemple, un suivi systématique de la mise en œuvre de ses dispositions en imposant, en son article 17, aux États de remettre tous les 6 ans un rapport sur l'application des dispositions prises, les rapports nationaux étant ensuite synthétisés par la Commission dans un rapport soumis aux États membres, au Parlement européen et au Conseil ${ }^{52}$. Il est d'ailleurs révélateur qu'une directive ${ }^{53}$ visant, spécifiquement en matière environnementale, à harmoniser et à rationaliser les procédures d'adoption des rapports, ait été adoptée. La création, dès 1992, d'un « réseau pour la mise en œuvre et le contrôle de l'application du droit de l'environnement de l'Union européenne $»^{54}$, appelé IMPEL témoigne également de l'importance de la réflexion relative au suivi du droit de l'environnement dans l'Union et d'ailleurs il n'est pas surprenant que IMPEL participe désormais à l'objectif du "Mieux légiférer » ${ }^{55}$. Ajoutons qu'en 2016, année de conclusion de l'accord «Mieux légiférer », la Commission a lancé l'examen de la mise en œuvre de la politique environnementale (Environmental Implementation Review «EIR »), un cycle de deux ans fondé sur l'analyse et le dialogue, dans le but d'améliorer l'application de la politique et de la législation européennes existantes en matière d'environnement dans le cadre duquel elle a publié sa première communication ${ }^{56}$.

Second exemple, la consultation des parties prenantes rejoint un principe général d'ouverture et de participation du public qui, dans les domaines de l'environnement et de la santé, est nettement valorisé. La conclusion par la Communauté ${ }^{57}$ de la Convention sur l'accès à l'information, à la participation du public au processus décisionnel et l'accès à la justice en matière d'environnement (dite «Convention d'Aarhus ») a, de ce point de vue, représenté un moment décisif dans l'énonciation de ce principe et surtout dans sa transcription sous la forme d'obligations. Un règlement, le règlement $\mathrm{n}^{\circ} 1367 / 2006$, a en effet été adopté précisément pour l'application aux institutions et organes de la

50 Conclusions de la Présidence, 25-26 juin 1990, http://europa.eu/rapid/press-release_DOC-90-2_fr.htm.

51 Directive 92/43 du 21 mai 1992 concernant la conservation des habitats naturels ainsi que de la faune et de la flore sauvages, fOCE du 22.07.1992, $\mathrm{n}^{\circ} \mathrm{L} 206$

52 Rapport de la Commission sur l'état de conservation des types d'habitats et des espèces conformément à l'article 17 de la directive «Habitats», 13 juillet 2009, COM (2009) 358 final - Non publié au fournal officiel. Voir plus récemment la Communication au Conseil et au Parlement européen accompagnée d'un rapport technique de l'Agence européenne pour l'environnement faisant le point sur l'évolution de l'état de conservation des centaines d'espèces et de types d'habitats naturels d'intérêt communautaire entre 2007 et 2012, ainsi que sur la contribution du réseau Natura 2000 à la réalisation des objectifs de la directive, COM (2015) 219 final.

53 Directive 91/692 du 23 décembre 1991, visant à la standardisation et à la rationalisation des rapports relatifs à la mise en ouvre de certaines directives concernant l'environnement, JOCE du 31.12.1991, $\mathrm{n}^{\circ} \mathrm{L} 377$.

54 IMPEL est une structure informelle composée de représentants d'organismes environnementaux ou d'agences de tous les États de l'Union (et de la Norvège, ainsi que de représentants des autorités publiques équivalentes dans les pays candidat). Ce réseau a vocation à permettre l'échange d'informations relatives à l'application et au contrôle du respect des législations sur l'environnement, et également le développement d'approches communes concrètes.

55 Par exemple, dans le cadre d'IMPEL, a été publié un rapport : M. Pallemaerts, P. Ten Brink, A. Farmer, D. Wilkinson, Developing a checklist for assessing legislation on practicability and enforceability, IMPEL, Brussels, 2007. Ce rapport a été utilisé pour examiner certaines propositions de révision de directives, par exemple la Directive 96/61/CE du Conseil du 24 septembre 1996 relative à la prévention et à la réduction intégrées de la pollution ou la Directive 2002/96/CE du Parlement européen et du Conseil du 27 janvier 2003 relative aux déchets d'équipements électriques et électroniques.

56 Communication, L'examen de la mise en ouvre de la politique environnementale de l'UE: défis communs et comment conjuguer nos efforts pour produire de meilleurs résultats, COM (2017) 63 final, le 3.02.2017 : « La présente communication s'accompagne d'une annexe et de 28 rapports EIR par pays. Ceux-ci décrivent les principaux défis et opportunités recensés par chaque État membre dans la mise en œuvre de la politique environnementale et se concentrent sur l'écart qui sépare les obligations juridiques et les accords politiques européens de la réalité du terrain. Les informations factuelles contenues dans les rapports ont été vérifiées par les États membres ».

57 Décision 2005/370/CE du Conseil, du 17 février 2005, relative à la conclusion au nom de la Communauté européenne, de la convention sur l'accès à l'information, la participation du public au processus décisionnel et l'accès à la justice en matière d'environnement, fO L 124 du 17.05 .2005 , p. $1-3$. 
Communauté des dispositions de la convention d'Aarhus ${ }^{58}$. Or, ce texte prévoit des obligations substantielles qui permettent tout autant de répondre à l'objectif de démocratie environnementale qu'à celui du « Mieux légiférer ». Son article 9 prévoit ainsi que « les institutions ou organes communautaires donnent au public, lorsque toutes les options sont encore possibles, une réelle possibilité de participer au plus tôt à l'élaboration, à la modification ou au réexamen des plans et programmes relatifs à l'environnement par le biais de dispositions pratiques et/ou autres voulues. » L'information du public se fait par des avis au public ou par d'autres moyens appropriés tels que les moyens de communication électroniques et doit porter notamment sur : le projet de proposition, les informations ou de l'évaluation environnementales disponibles relatives au plan ou programme en cours d'élaboration, les détails relatifs aux modalités de participation. Sauf circonstance particulière, un délai de huit semaines au moins doit être laissé pour la présentation d'observations. Il est évidemment prévu que les institutions et organes de l'Union tiennent dûment compte du résultat de la participation du public qui doit être informé des motifs et des considérations fondant la décision. On fera d'ailleurs remarquer la parenté entre ce processus et celui prévu dans le nouveau programme ${ }^{59}$ sur le « Mieux légiférer $»$ puisque l'une ${ }^{60}$ des nouveautés est qu'une consultation publique de... $8 \ldots$ semaines sera désormais mise en œuvre lorsqu'une proposition aura été adoptée par la Commission ; les contributions recueillies au cours de cette consultation seront transmises au Parlement et au Conseil qui pourront, dès lors, les intégrer dans leurs travaux.

Les domaines de l'environnement et de la santé ne se contentent pas toutefois d'offrir des exemples (dont nous n'avons donné qu'un aperçu non exhaustif) d'une mise en application des principes du «mieux légiférer». Ils permettent, et c'est sans doute ce qui est le plus intéressant, d' « éprouver » ces principes dans la mesure où, en ces matières, ils sont clairement mis en tension.

\section{2) La mise en tension des principes du «Mieux légiférer »}

À première vue, « le programme «Better regulation » propose des mesures qu'il est en apparence difficile de blâmer. Il est légitime de soutenir l'ajout de processus de consultation publique, la simplification [...] ou encore la prise en compte de la faisabilité d'une action publique dans sa conception, s'il est prouvé qu' in fine ces mesures permettront d'améliorer l'efficacité des politiques européennes » ${ }^{61}$. Cela l'est d'autant plus lorsque ces mesures s'imbriquent facilement aux principes structurants comme c'est le cas en matière d'environnement et de santé.

\footnotetext{
58 Règlement (CE) $\mathrm{n}^{\circ}$ 1367/2006 du Parlement européen et du Conseil du 6 septembre 2006 concernant l'application aux institutions et organes de la Communauté européenne des dispositions de la convention d'Aarhus sur l'accès à l'information, la participation du public au processus décisionnel et l'accès à la justice en matière d'environnement, fO L 264 du 25.09.2006, p. 13-19.

59 Communication de la Commission au Parlement européen et au Conseil, Améliorer la réglementation pour obtenir de meilleurs résultats - Un enjeu prioritaire pour l'UE, COM (2015) 215 final.

60 Une autre nouveauté est que « toutes les parties intéressées pourront fournir des informations en retour sur les actes qui fixent les éléments spécifiques ou techniques nécessaires pour mettre en oeuvre la législation adoptée par le Parlement européen et le Conseil », soit les actes délégués et actes d'exécution qui seront accessibles au grand public pendant quatre semaines ce qui permettra aux parties intéressées de formuler leurs observations.

61 C. De Marcilly, M. Touillon, « Programme «Mieux légiférer» : l'expertise surpasse-t-elle le politique ? » Question d'Europe ${ }^{\circ} 370$, 9.11.2015.
} 
Toutefois, à y regarder de plus près, le programme « Mieux légiférer » n'est pas totalement insusceptible d'interrogations, voire de critiques. Et pour cause, les rationalités ou logiques à l'œuvre dans le cadre de ce programme - la logique simplificatrice (2.1) tout autant que la logique expertale (2.2) peuvent susciter des interrogations (et parfois même des critiques) quant aux risques qui y sont associés. Les domaines de l'environnement et de la santé ont ceci de particulier qu'ils révèlent de manière vive de telles interrogations et qu'ils permettent d'en éprouver les risques (et les réponses possibles).

\subsection{La mise en tension de la logique simplificatrice}

La logique simplificatrice du « Mieux légiférer » peut être résumée de la façon suivante en reprenant une formule de la Commission : « Une réglementation bien ciblée, fondée sur des données probantes et rédigée de manière simple aura plus de chances d'être correctement mise en ouvre et d'atteindre ses objectifs sur le terrain, qu'ils soient d'ordre économique, social ou environnemental $»^{62}$. A priori non critiquable, une telle logique se heurte pourtant à des objections importantes que les domaines qui nous occupent permettent d'illustrer. Parfois, les objections concernent les outils mobilisés en ce but qui, sur le plan de la méthode, peuvent susciter des questions non négligeables. Parfois les objections sont d'ensemble, relatives directement à l'objectif de simplification de la réglementation.

S'agissant des outils mobilisés, il faut admettre que l'outil qu'est l'analyse d'impact suscite des interrogations substantielles. L'une d'entre elle, majeure, concerne la méthode pertinente à retenir pour effectuer telle analyse. La Commission souligne, dans sa Communication fondatrice sur l'analyse d'impact, que «plusieurs méthodes d'analyse peuvent être utilisées pour évaluer l'impact. Elles diffèrent au niveau du concept et du champ d'application (par exemple : analyse coûts-avantages, analyse coût-efficacité, analyse des coûts de mise en conformité, analyse multicritères et évaluation des risques). Mais elle rappelle que, quelle que soit la méthode, «pour faciliter la comparaison entre les différents impacts et identifier les compromis et les situations avantageuses pour tous de manière transparente, il est souhaitable de quantifier l'impact en termes matériels et, le cas échéant, en termes monétaires (en plus de l'appréciation qualitative) ${ }^{63}$. Dans ses lignes directrices pour l'analyse d'impact, la Commission réitère la même exigence considérant que, pour permettre des analyses approfondies, il convient « de produire une estimation quantitative/monétaire des coûts et bénéfices escomptés » ajoutant même de manière pour le moins abrupte : « si la quantification/ monétisation est impossible, justifier $»^{64}$ ! Certes, dans ces documents, il est admis que les effets puissent être «non traduisibles en termes quantitatifs ou monétaires » et que pour cette raison, « ils ne doivent pas être jugés moins importants étant donné qu'ils peuvent contenir des aspects intéressants pour la décision politique $»^{65}$. D’ailleurs, dans les lignes directrices, la Commission prévoit que

62 Communication de la Commission, Améliorer la réglementation : de meilleurs résultats pour une Union plus forte, 14 septembre 2016, COM 2016 (615) final.

63 Communication de la Commission sur l'analyse d'impact, COM (2002) 0276 final, annexe 1, pt 4-2.

64 Commission européenne, Lignes directrices concernant l'analyse d'impact, SEC (2009) 92, p. 43.

65 Communication de la Commission sur l'analyse d'impact, COM (2002) 0276 final, annexe 1, pt 4-2. 
pourra être réalisée une « analyse multicritère » « lorsque l'on traite des cas nécessitant de présenter des incidences mêlant données qualitatives, quantitatives et monétaires et présentant divers degrés de certitude $»^{66}$. Toutefois, la quantification notamment monétarisée des impacts demeure, du point de vue de la Commission, centrale dans l'analyse d'impact. Et d'ailleurs, elle a souvent procédé ainsi y compris dans les domaines de l'environnement et de la santée ${ }^{67}$.

La difficulté est pourtant que certains impacts sont rétifs à une telle logique de la quantification et de la monétarisation. C'est le cas de nombre d'impacts environnementaux et sanitaires. Les débats ${ }^{68}$ autour de la possibilité d'attribuer un coût à la biodiversité et de mener une évaluation économique des services de la biodiversité en témoignent. Ils portent tout autant sur la légitimité d'attribuer un coût à la biodiversité ${ }^{69}$ que sur sa faisabilité ${ }^{70}$. On retrouve de telles objections s'agissant des impacts sur la santé puisqu'il s'agit in fine de mesurer la réduction de la morbidité et de la mortalité ce qui impose d'attribuer une «valeur de la vie humaine $»^{71}$. Or, outre la difficulté théorique ${ }^{72}$, une telle attribution a pu faire débat d'un point de vue empirique notamment car le critère de la réduction de la mortalité paraît insuffisant pour intégrer correctement dans les évaluations socio-économiques l'ensemble des enjeux relatifs au risque santé ${ }^{73}$. La conséquence est qu'il est parfois extrêmement difficile, dans ces domaines, d'estimer les impacts des règlementations envisagées. La Commission fait preuve d'ailleurs parfois d'une grande transparence en ce qui concerne les incertitudes et du coup les limites associées à la méthodologie utilisée dans l'analyse d'impact ${ }^{74}$. L'analyse d'impact accompagnant la proposition de directive concernant la qualité de l'essence et des carburants diesel et la promotion de l'utilisation de l'énergie produite à partir de sources renouvelables en atteste. La Commission reconnaît que l'estimation des impacts de la réglementation envisagée (en faveur

66 Commission européenne, Lignes directrices concernant l'analyse d'impact, SEC (2009) 92, p. 44.

67 L'exemple de la Recommandation du Conseil de 2009 relative aux environnements sans tabac et de l'analyse d'impact réalisé par la Commission au soutien de sa proposition de recommandation (Document de travail des services de la Commission accompagnant la proposition de Recommandation du Conseil relative aux environnements sans tabac, Résumé de l'analyse d'impact, COM (2008) 328 final) est emblématique car dans cette analyse, l'effort de quantification est tout à fait manifeste. L'analyse d'impact est structurée autour de la comparaison d'options du point de vue du niveau d'exposition à la fumée de cigarette dans certains lieux (comme les bars, les restaurants, les bureaux et les lieux publics). Or, le raisonnement de la Commission est clairement de «calculer » l'impact des options politiques sur la réduction de la mortalité, en y faisant correspondre, pour la monétiser, des coûts médicaux et des coûts non médicaux associés à ces maladies.

68 M. Hautereau-Boutonnet et E. Truilhé-Marengo, "Recherche interdisciplinaire sur les valeurs de la biodiversité - Acte 1 », in S. Lacour, O. Leclerc, L. Dumoulin (éd.), Regards croisés sur les objets et les pratiques scientifiques et techniques, Cahiers Droit Sciences \& technologies , n 6 (2016), PUAM, p. 145-163.

69 La question est la suivante : la biodiversité n'est-elle pas protégeable en soi pour ce qu'elle est, en dehors de toute évaluation monétaire ? Les critiques bien évidemment dépassent le cadre économique et sont philosophiques (la biodiversité est-elle commensurable ?) ou éthiques (est-il légitime de donner un prix à la nature ?) et finalement idéologiques.

70 La principale difficulté rencontrée par ces évaluations est de pouvoir mesurer les bénéfices environnementaux associés aux usages non marchands tels que les activités récréatives en rapport avec la biodiversité, ou le simple fait de bénéficier d'un paysage naturel à proximité de chez soi, ce qui peut conduire à sous-évaluer les bénéfices globaux.

71 La référence d'une valeur de la vie humaine (valeur de la vie statistique (VVS)) est régulièrement utilisée dès lors qu'on cherche à apprécier l'impact d'une dépense, d'une réglementation ou encore d'un investissement sur le risque santé. Elle traduit l'effort que la collectivité est prête à consentir pour réduire le risque de décès de la population et permet de comparer les coûts de la réduction du risque aux avantages attendus en termes de vies sauvées.

72 L'idée d'associer une valeur monétaire à la vie humaine est par principe et philosophiquement discutable car, la vie n'a pas de prix, au moins du point de vue complexe qui est celui de l'individu.

73 Le référentiel permet en effet uniquement d'appréhender les années de vie gagnées sans prendre en compte la qualité de vie. C'est la raison pour laquelle ont été développés d'autres indicateurs tels que «QALY » ou «DALY » qui peuvent agréger de façon consensuelle la quantification des années de vies perdues ou altérées par une qualité de vie dégradée.

74 Première évaluation dans le cadre du Parlement européen d'une analyse d'impact de la Commission européenne - Proposition de la Commission européenne d'une directive sur les changements indirects d'affectation des sols liés aux biocarburants et aux bioliquides. Analyse d'impact [SWD (2012) 343 final, SWD (2012) 344 final (résumé)] portant sur une proposition de la Commission d'une directive du Parlement européen et du Conseil modifiant la directive 98/70/CE concernant la qualité de l'essence et des carburants diesel et modifiant la directive 2009/28/CE relative à la promotion de l'utilisation de l'énergie produite à partir de sources renouvelables (COM (2012) 595 final). 
des biocarburants) est affectée d'un niveau élevé d'incertitude ${ }^{75}$ et que dans ce contexte, elle ne peut être uniquement de nature qualitative. D'ailleurs, le comité d'analyse d'impact avait critiqué la démarche et avait demandé à la Commission de produire un effort supplémentaire pour quantifier les incidences escomptées... La conséquence associée est que, dans l'analyse d'impact, la prise en compte des différents impacts ou «piliers $\gg^{76}$ n'est pas assurée d'être toujours équivalente. Dans son rapport sur l'analyse d'impact, la Cour des comptes européenne a d'ailleurs souligné une telle asymétrie entre les trois «piliers », ainsi qu'entre les coûts et les avantages ${ }^{77}$. Nombre d'études de lobbies environnementaux ont également conclu à une « dé-prioritisation ${ }^{78} \mathrm{du}$ " pilier environnemental » dans l'analyse d'impact et tenté de convaincre la Commission de ré-équilibrer l'évaluation des impacts.

Au-delà des objections sur les outils, une objection concerne l'objectif par lui-même : soit celui de la simplification de la règle de droit. Il peut en effet être reproché à un tel objectif d'être en partie illusoire. «La complexité des normes juridiques est le reflet de la complexité des rapports sociaux que le droit a pour fonction de réguler : comment imaginer que le droit puisse être simple alors que la société apparaît de plus en plus complexe ? Et la diversité des intérêts qu'il est appelé à concilier est inévitablement source de complexité $»^{79}$. Dans le domaine de l'environnement et de la santé, l'illusion apparaît très franchement. Dans ces domaines souvent complexes (au plan scientifique et technique notamment), les règles en la matière doivent, pour être opérationnelles, l'être aussi ce qui ne satisfait pas dès lors à l'exigence de simplicité. À l'appui, on citera à nouveau le règlement REACH qui, avec ses 879 pages et ses libellés très techniques ${ }^{80}$, est pour le moins éloigné de l'objectif de simplicité de la règle. On ajoutera, en plus, que la logique d'une réglementation simple et fondée sur des données probantes n'est pas plus adaptée en ces matières. Les dommages suspectés sont souvent incertains, leur réalisation est simplement plausible et il est donc difficile de collecter, au soutien de l'adoption d'une mesure de protection, des données «probantes $»^{81}$. D'ailleurs, depuis

75 C'est le cas s'agissant des estimations relatives aux effets des changements indirects d'affectation des sols sur les émissions de gaz à effet de serre. Les émissions de gaz à effet de serre dues au changement indirect d'affectation des sols (CIAS) se produisent lorsque les cultures ou les terres qui, autrement, auraient été utilisées pour produire des denrées alimentaires ou des aliments pour animaux sont utilisées pour la culture de biocarburants et que la production agricole existante est déplacée géographiquement vers de nouvelles zones d'exploitation créées par conversion d'espaces naturels.

76 Communication de la Commission au Conseil et au Parlement européen, Améliorer la réglementation en matière de croissance et d'emploi dans l'Union européenne (COM (2005) 0097 final) : «Si l'outil d'évaluation d'impact fournit une base solide, la Commission estime que l'évaluation des répercussions économiques doit être renforcée de façon à contribuer aux objectifs de la nouvelle stratégie de Lisbonne. Le renforcement du pilier économique de l'évaluation d'impact ne compromet nullement l'importance du développement durable et de l'approche intégrée, qui reste le fondement de la stratégie de la Commission ».

77 Cour des comptes européenne, L'analyse d'impact dans les institutions européennes: soutient-elle la prise de décision ?, Rapport spécial $n^{\circ} 3 / 2010$.

78 Ragnar E. Lofstedt, The 'Plateau-ing' of the European Better Regulation Agenda: An analysis of activities carried out by the Barroso Commission, disponible à l'adresse : https://pdfs.semanticscholar.org/989d/8662c25fb7af3f482e50ba99a3a343a3727c.pdf.

79 J. Chevallier, « La simplification de l'action publique et la question du droit », Revue française d’administration publique, 1/2016 ( $\left.\mathrm{N}^{\circ} 157\right)$, p. 205-214.

80 Pour donner un exemple précis, le champ d'application du règlement prévoit ce qui est couvert par l'obligation d'enregistrement et $a$ contrario ce qui est exempté. Or, sont par exemple exemptés les «polymères ». Selon le règlement, les polymères sont des substances de masse molaire élevée dont la structure résulte essentiellement de la répétition d'unités de faible masse molaire (monomères). Étant donné que les polymères sont composés de monomères qui doivent être enregistrés, ils présentent un risque limité et sont donc exemptés d'enregistrement et d'évaluation. La question est toutefois de savoir ce qu'est un monomère ce que ne dit pas le texte. Il n'est d'ailleurs pas étonnant au vu de cette exemption que la Cour ait été sollicitée pour délimiter plus précisément ladite notion : CJCE, 7 juillet 2009, The Queen, à la demande de S.P.C.M. SA, C.H. Erbslöh KG, Lake Chemicals and Minerals Ltd et Hercules Inc. c/ Secretary of State for the Environment, Food and Rural Affairs, aff. C-558/07, Rec. 2009 I-05783.

81 C. Noiville, N. De SADELEER, «La gestion des risques écologiques et sanitaires à l'épreuve des chiffres, Le droit entre enjeux scientifiques et politiques », RDUE, 2/2001, p. 389-447. 
longtemps, il est admis, par la Cour que, « lorsque des incertitudes subsistent quant à 1'existence ou à la portée des risques pour la santé des personnes, les institutions communautaires peuvent prendre des mesures de protection sans avoir à attendre que la réalité et la gravité de ces risques soient pleinement démontrées ${ }^{82}$. Une telle faculté a été également consacrée dans le traité dans le domaine de l'environnement ${ }^{33}$.

\subsection{La mise en tension de la logique expertale}

La mise en tension concerne également une autre logique qui sous-tend clairement le « Mieux légiférer », soit la logique « expertale » c'est-à-dire le développement dans le processus décisionnel de la place des experts (et la dépolitisation subséquente). C'est notamment au travers des discussions sur la portée de l'analyse d'impact dans le processus décisionnel qu'une telle mise en tension s'est manifestée. Elle peut s'expliquer ainsi. Certes, à première vue, l'analyse d'impact est un processus interne et sous la responsabilité d'une autorité politique, la Commission, depuis sa planification $^{84}$ jusqu'à sa formalisation et son contrôle de sa qualité ${ }^{85}$. De plus, l'analyse réalisée a vocation uniquement à être « un outil d'aide à la décision et ne remplace pas l'appréciation politique ${ }^{86}$. Toutefois, pour élaborer l'analyse d'impact, consciente des limites de ses ressources, la Commission organise souvent un recours à l'information extérieure et fait notamment appel à des contractants privés pour conduire des analyses et procéder à des consultations. En plus, pour répondre à des critiques de partialité, le contrôle de la qualité de ces analyses d'impact a été ouvert au-delà de la Commission ${ }^{87}$. Enfin, dans la pratique, l'analyse d'impact pèse parfois de manière tout à fait déterminante sur la décision finale.

On soulignera que les domaines de l'environnement et de la santé ont permis de mettre en exergue ces discussions. Ils ont notamment permis au juge de l'Union de s'exprimer en la matière et de rappeler la portée limitée des analyses d'impact, énonçant qu'elles ne sont obligatoires ni pour les co-législateurs, ni pour la Commission elle-même, bref qu'elles ne « sauraient avoir un caractère d'automaticité et de prévalence sur l'appréciation politique $»^{88}$. Ainsi, dans son arrêt du 16 décembre 2015, Suède c/ Commission ${ }^{89}$, le Tribunal a considéré que la Commission avait manqué aux obligations

82 CJCE, 5 mai 1998, Royaume-Uni c./ Commission, Rec. 1998 I2265.

83 Voir l'article 191 § 2 TFUE qui prévoit que « la politique de l'Union dans le domaine de l'environnement [...] est fondée sur les principes de précaution et d'action préventive ».

84 «La décision de conduire une étude d'impact des réglementations appartient à la Commission. Tout au plus, l'absence d'étude d'impact peut mettre la Commission dans la situation d'être confronté à un a priori négatif du juge et devrait se justifier sur les moyens mis en œuvre pour respecter le caractère proportionnel de la mesure envisagée [...] Seul le Médiateur européen a contrôlé le comportement de la Commission dans la conduite des études d'impact, comme cas potentiel de mauvaise administration. Cependant, le contrôle est limité à l'erreur manifeste d'appréciation » : T. Delille, « La dimension interinstitutionnelle des études d'impact des réglementations dans l'Union européenne à l'ère de la « réglementation intelligente » », Revue française d'administration publique, 1/2014 (n 149), p. 61-77.

85 Pour des détails : voir T. Delille, « La dimension interinstitutionnelle des études d'impact des réglementations dans l’Union européenne à l'ère de la « réglementation intelligente » », ibidem.

86 Communication de la Commission sur l'analyse d'impact, COM (2002) 0276 final, pt 1-2.

87 Le Comité d'analyse d'impact (CAI) a été remplacé par le comité d'examen de la réglementation constitué d'un président et de 6 membres dont 3 sont recrutés hors institutions de l'Union ce qui atteste de la volonté de la Commission d'ouvrir la «cuisine interne» pour reprendre la métaphore de Frans Timmermans et de réduire la perception que les services de la Commission chargés d'un projet sont également responsables de leur étude d'impact.

88 E. VAN DEN ABEELE, «Mieux légiférer » : une simplification bureaucratique à visée politique, Working Paper 2015. 04 , p. 46.

89 Trib., 16 décembre 2015, Royaume de Suède c./ Commission européenne, aff. T-521/14, ECLI:EU:T:2015:976. 
qui lui incombent en vertu du règlement (UE) n ${ }^{\circ}$ 528/2012 du Parlement européen et du Conseil, du 22 mai 2012, concernant la mise à disposition sur le marché et l'utilisation des produits biocides, en s'abstenant d'adopter des actes délégués en ce qui concerne la spécification des critères scientifiques pour la détermination des propriétés perturbant le système endocrinien ${ }^{90}$. Or, il faut noter que l'un des arguments avancés par la Commission afin de justifier la non-adoption de ceux-ci avait été précisément trouvé dans la nécessité de procéder à une analyse d'impact (analyse d'impact des critères scientifiques retenus et des différentes solutions de substitution à ces critères). Toutefois, le Tribunal considère qu'aucune disposition du droit de l'Union « n'exige une telle analyse d'impact » ${ }^{91}$ et que, de surcroît, à supposer que la Commission doive procéder à une telle analyse d'impact, cela ne l'exonérait en rien de respecter la date fixée par le règlement. On notera que, à la suite de cet arrêt en carence, la Commission a publié sa proposition, en réalisant en amont une analyse d'impact qu'elle n'a d'ailleurs suivie qu'à moitié ${ }^{92}$. Dans l'affaire $A f t o n^{93}$ dans laquelle la Cour avait à connaître d'une question préjudicielle en appréciation de la validité des dispositions d'une directive fixant des limites quantitatives à l'utilisation d'additifs dans les carburants ${ }^{94}$, la Cour avait précédemment et fermement affirmé que l'analyse d'impact réalisée par la Commission ne lie pas le Conseil et le Parlement qui sont, en tant que co-législateurs, également « en droit d'apporter des modifications à ladite proposition $»^{95}$ sous réserve de fonder leur délibération sur d'autres documents scientifiques avancés pendant la procédure législative ${ }^{96}$.

Ces arrêts rejoignent d'ailleurs une abondante jurisprudence dans le domaine de l'environnement et de la santé relative au pouvoir d'appréciation qu'il convient, en ces domaines, de réserver aux autorités politiques. Il est en effet affirmé de façon constante, par le juge de l'Union, qu'il appartient aux autorités politiques, notamment aux institutions de l'Union de fixer librement le niveau de protection de l'environnement et de la santé qu'elles estiment approprié pour la société. C'est la raison pour laquelle, le juge de l'Union insiste bien sur le fait que les avis scientifiques rendus - s'ils doivent être bien et intégralement ${ }^{97}$ pris en considération - ne lient pas les institutions qui ne sont en aucune manière tenues de suivre les conclusions exprimées ${ }^{98}$ dont elles peuvent se départir lorsqu'elles considèrent que le seuil de l'acceptabilité des effets potentiels adverses est dépassé et que le recours à des mesures préventives est nécessaire. Selon le juge de l'Union, « cette conclusion se

\footnotetext{
$90 \quad$ Pts 53-54 de l'arrêt.

91 Pt 74 de l'arrêt.

92 Voir Communication de la Commission au Parlement et au Conseil sur les perturbateurs endocriniens et les projets d'actes de la Commission visant à définir des critères scientifiques pour leur détermination dans le cadre de la législation de l'UE relative aux produits phytopharmaceutiques et aux produits biocides, COM (2016) 350 final Bruxelles, 15.06.2016. L'une des questions qui se posait était celle de savoir si la puissance d'une substance chimique (soit sa capacité à produire un effet à une dose donnée) devait être intégrée pour définir de tels perturbateurs. Il est rappelé dans la Communication que le rapport d'analyse d'impact aborde les questions liées à la puissance et recense les arguments à l'appui de la prise en compte de ce facteur. Néanmoins, la Commission décide toutefois de suivre le large consensus scientifique selon lequel la puissance ne devrait pas être prise en compte lors de l'identification des perturbateurs endocriniens, mais qu'elle devrait l'être lors de l'évaluation du risque réel que les perturbateurs endocriniens pourraient représenter.

93 CJUE, 8 juillet 2010, Afton, aff. C-343/09, Rec. 2010 I-07027.

94 La société Afton arguait que le Parlement et le Conseil avait commis une erreur manifeste d'appréciation en fixant lesdites limites. À cette fin, Afton observait « que l'imposition de ces limites n'est pas appuyée par la conclusion de la Commission formalisée dans l'étude d'impact jointe à la proposition de directive et que lesdites limites sont impraticables et arbitraires » (Pt 29 de l'arrêt).

95 Pt 30 de l'arrêt.

96 Pts 36-37.

97 TPICE, ordonnance, 28 septembre 2007, République Française c./ Commission, aff. T-257/07, Rec. p. II-4153, pt 73.

98 Voir l'arrêt Trib., 9 décembre 2011, Dow AgoSciences Ltd c. Commission, aff. T-475/07, Rec. 2011 II-05937 qui reprend les jurisprudences passées.
} 
justifie par des considérations de principe relatives à la responsabilité politique et à la légitimité démocratique de la Commission. «Alors que l'exercice de l'autorité publique par la Commission est légitimé [...] par le contrôle politique du Parlement européen, les membres (des comités scientifiques ou des agences), s'ils disposent d'une légitimité scientifique, n’ont pas de légitimité démocratique ni de responsabilité politique. Or, une légitimité scientifique ne suffit pas pour justifier l'exercice de l'autorité publique ${ }^{99} \ldots$ Dans le même temps, et en dépit de ces fermes rappels jurisprudentiels, les domaines de l'environnement et de la santé permettent d'illustrer combien, en pratique, la séparation entre l'expertise et la décision politique est bien moins simple qu'il n'y paraît et infiniment plus poreuse $^{100}$. Dans la pratique en effet, les avis de certains comités ou de certaines agences (ou les informations produites par ces avis) exercent une influence importante, déterminante bien souvent sur la décision finale, notamment dans des domaines de fortes controverses publiques comme ceux relatifs à l'environnement et la santé. Les rapports par exemple entre la Commission et certaines de ses agences, l'Autorité européenne de sécurité des aliments ${ }^{101}$ ou encore l'Agence européenne du médicament ${ }^{102}$ sont tout à fait illustratifs dans la mesure où les décisions de la Commission ont, à quelques exceptions près, toujours suivi les avis de ces agences. On voit ainsi se profiler de nombreuses hypothèses «d'expertise instituante », dans laquelle « le savoir de l'expert produit directement un ordre de normes et de règles juridiques $»^{103}$ sans que les mécanismes de contrôle ne soient adaptés à une telle configuration ${ }^{104}$.

Pour conclure, il ne fait guère de doute qu'il y a un net intérêt à considérer, pour explorer les principes du « Mieux légiférer », le droit de l'Union européenne de l'environnement et de la santé. Et pour cause, le droit en ces matières est tout à la fois structurellement tout à fait accueillant vis-à-vis desdits principes mais également souvent rétif à leur logique. En cela, le domaine de l'environnement et de la santé permet, sans doute plus que d'autres, de mettre en perspective l'importante ambivalence de l'initiative. En effet, l'initiative «Mieux légiférer» poursuit clairement « au-delà des objectifs affichés, des considérations propres à la Commission et liées notamment

99 TPICE, 11 septembre 2002, Pfizer Animal Health SA c/ Conseil, aff. T-13/99, Rec., p. II-03305, pt. 199.

100 C. Noiville, N. DE SADELEER, «La gestion des risques écologiques et sanitaires à l'épreuve des chiffres, Le droit entre enjeux scientifiques et politiques », RDUE, 2/2001, p. 389-447

101 Voir sur ce sujet : E. Brosset, L’Autorité européenne de sécurité des aliments et la dissémination d'OGM in F. CouzINET (éd.), La création de l'Autorité européenne de sécurité des aliments : enjeux et perspectives, IREDE, Université de Toulouse, p. 101-149.

102 Pour une vue plus fine voir B. Hauray, " Politique et Expertise scientifique. L'évaluation européenne des médicaments », Sociologie du travail, vol 47 (1), janvier-mars 2005, p. 57-66. «Entre 1995 et 2002, un seul avis du CSP a été renvoyé par la Commission à l'EMEA afin que le CSP précise ses motivations (cet avis concernait le retrait des médicaments coupe-faim) [...] et un seul avis du CSP a été modifié suite au passage » devant les représentants des États (à propos du Viagra).

103 R. CASTEL, «Savoirs d'expertise et production de normes » in F. Chazel, J. Commaille (éd.), Normes juridiques et régulation sociale, Droit et société (1987), p. 177-178.

104 Par exemple, les voies de contrôle, politique et juridictionnel, des actes pris par les agences ne sont pas toujours adaptées. Par exemple, aucun recours n'est prévu à l'encontre des actes de l'Autorité européenne de sécurité des aliments ou de l'EAM devant la Cour. Lorsqu'il s'agit d'actes ou de décisions dont l'élaboration s'effectue en plusieurs phases, notamment au terme d'une procédure interne, ne constituent, en principe, des actes susceptibles de faire l'objet d'un recours en annulation que les mesures qui fixent définitivement la position de l'institution. Ne sont donc pas logiquement attaquables les actes préparatoires puisqu'ils ne constituent pas une manifestation définitive de volonté ce que le Tribunal a confirmé à propos des avis scientifiques de l'EFSA. Trib., ord., 17 juin 2008, FMC Chemical c./ EFSA, T-311/06, Rec. 2008, p. II-88, pt 43 et T-312/06, Rec. 2008, p. II-89, pt. 43 et Dow AgroSciences/ EFSA, T-397/06, Rec. 2008, p. UU-90, pt. 40. 
à la quête de légitimité de cette dernière $»^{105}$. Au-delà de leur intérêt en tant que tels, de tels principes ont sans aucun doute pour vocation d'asseoir une forme légitimité de l'action de l'Union et spécialement de la Commission, une légitimité par l'efficacité de l'action ${ }^{106}$. Or, si dans ses objectifs premiers, le programme « Mieux légiférer » coïncide avec des objectifs plutôt consensuels déjà poursuivis dans le cadre de la politique de l'Union en matière d'environnement et de santé, s'agissant de cet objectif indirect, il suscite au contraire des questions bien plus nombreuses que la politique en matière d'environnement et de santé permet clairement de mettre en lumière. Et pour cause, la question de(s) facteur(s) de la légitimité dans l'Union demeure, doit-on le rappeler, pour le moins débattue ${ }^{107}$ et le « Mieux légiférer » ne permettra pas d'en faire l'économie, bien au contraire.

105 C. De Marcilly, M. Toulllon, « Programme "Mieux légiférer" : l'expertise surpasse-t-elle le politique ? » Question d’Europe n 370, 9.11.2015.

106 V. Schmidt, Democracy and legitmacy in the European Union revisited: Input, Output and «Throughput », Political Studies 2013 vol. 61, 2-22, disponible à l'adresse : http://journals.sagepub.com/doi/pdf/10.1111/j.1467-9248.2012.00962.x.

107 Deux «modèles » de légitimité coexistent, celui de la légitimité par la participation et celui de la légitimité par les résultats. Dans l'Union, le second a souvent eu les faveurs car il correspondait à l'architecture institutionnelle de l'Union et permettait de répondre aux critiques de déficit démocratique. Toutefois, il ne semble pas possible (et souhaitable) d'évacuer le premier qui doit coexister. Sur cette question, pour une vue d'ensemble et des indications bibliographique : A. BAILlEux, H. Dumont, Le pacte constitutionnel européen, tome 1, 2015, 568 p., spéc. p. 445-497. 



\title{
LA POLITIQUE SOCIALE À L'AUNE DU « MIEUX LÉGIFÉRER »
}

\author{
Dominique NAzet-Allouche ${ }^{1}$
}

\begin{abstract}
Résumé
La nouvelle politique du « Mieux légiférer » est pourvue d'un impact significatif sur la politique sociale, du fait de l'abandon de précédentes propositions de directives, de la réticence induite à promouvoir de nouvelles normes, de la multiplication des analyses d'impact affectant le processus décisionnel. Le socle européen des droits sociaux récemment adopté et ses initiatives subséquentes ne suffiront pas à compenser les effets négatifs du « Mieux légiférer » au regard du renforcement des droits des travailleurs.
\end{abstract}

\section{Abstract}

The Better Regulation Agenda has a significant impact on social policy, due to the abandonment of previous proposals for directives, the reluctance to promote new standards, the multiplication of impact assessments about the European policy-making. The recent European Pillar of social rights and its subsequent initiatives will not be able to reduce the negative effects of Better regulation for the workers'rights.

Dernière étape en date d'une politique enclenchée au début des années 2000, le paquet « Mieux légiférer » publié par la Commission européenne le 19 mai 2015, complété par l'accord interinstitutionnel du 13 avril 2016 et la communication de la Commission du 14 septembre 2016, affichent pour objectif d'améliorer l'efficacité du processus législatif de l'Union européenne.

Ce programme est pourvu de trois ressorts : l'amélioration de la transparence du processus décisionnel, le recours à une expertise probante, ainsi que l'amélioration et la simplification de la réglementation européenne. Appliquées à la politique sociale, de telles options, et notamment la dernière, ne pourraient qu'être approuvées si elles devaient conduire à une réglementation renforcée et judicieuse améliorant les droits des travailleurs, d'application et d'interprétation plus aisées. Toutefois, la finalité sous-jacente de la stratégie du « Mieux légiférer », à savoir favoriser le

1 Chercheure, Aix Marseille Univ, Université de Toulon, Univ Pau \& Pays Adour, CNRS, DICE, CERIC, Aix-en-Provence, France. 
développement des entreprises, autant que sa mise en œuvre telle que proposée par la Commission, conduisent à une appréciation beaucoup plus réservée voire critique, notamment de la part des organisations syndicales. Ainsi, la Confédération européenne des syndicats affirme que l'initiative Better Regulation est un programme de déréglementation visant à démanteler l'acquis social européen². Les programmes de travail de la Commission pour les années 2016 et 2017 sont sans aucun doute imprégnés du programme REFIT et de la politique du « Mieux légiférer ». Ainsi, l'année 2016 n'a connu aucune avancée en matière sociale.

Pourtant, à coté de la volonté de mieux légiférer, s'est récemment manifestée l'intention de redynamiser l'Europe sociale, en panne depuis un certain nombre d'années. En effet, le vote en faveur $\mathrm{du}$ «brexit » comme la montée de partis populistes et eurosceptiques, favorisée par le sentiment de délaissement de la part des classes populaires, incitent les institutions européennes à rafraîchir la coloration sociale de l'Europe.

Dans quelle mesure ces deux objectifs sont-ils conciliables et conciliés ? Vont-ils cohabiter ou au contraire l'un prend-il et prendra-t-il le pas sur l'autre? Quelle est pour l'heure la teneur des projets de la Commission en matière sociale ? Force est de constater que le programme de travail pour 2017 ne s'avère guère audacieux.

\section{1) Le social à l'épreuve du «Mieux légiférer»}

Mettant en œuvre sa volonté de Mieux légiférer, la Commission a renoncé à quatre-vingts propositions normatives non finalisées dans son programme de travail pour 2015. Parmi elles, deux relèvent du domaine social : il s'agit de la révision de la directive 92/85 sur la protection de la maternité et d'une proposition de directive relative à la représentation des femmes dans les conseils des sociétés, lesquelles avaient suscité soit un blocage au niveau du Conseil, soit un désaccord entre Parlement et Conseil. La Commission a mis en œuvre à leur propos son option de « considérer comme une bonne pratique de gestion législative le fait de retirer des propositions bloquées en phase législative ». D’autres propositions ont ensuite été officiellement abandonnées ou sont tombées dans les limbes : une politique qui ne paraît pas étrangère à la politique du « Mieux légiférer ». En outre, le social n'est pas épargné par la multiplication des analyses d'impact, outil destiné à l'amélioration des normes de l'Union.

2 https://www.etuc.org/fr/issue/better-regulation. 


\subsection{L'abandon des propositions précédemment émises}

La révision de la directive $\mathbf{9 2 / 8 5 ^ { 3 }}$ sur la protection de la maternité. En 2008, la Commission a proposé une révision de la directive du 19 octobre 1992 afin, entre autres, de porter le congé de maternité de 14 à 18 semaines. En octobre 2010, le Parlement européen s'est, notamment, exprimé en faveur d'un congé maternité de 20 semaines intégralement payé, et d'un congé paternité de 2 semaines. La proposition est ensuite restée bloquée au Conseil pendant plus de 4 ans. Après avoir donné, en décembre 2014, six mois supplémentaires aux co-législateurs pour trouver un accord, la Commission a abandonné sa proposition le $1^{\text {er }}$ juillet 2015, retrait précédemment annoncé dans la communication REFIT de 2014 et confirmé dans le programme de travail de la Commission pour 2015 , tout en annonçant la présentation ultérieure d'une proposition de plus grande ampleur ${ }^{5}$.

Le 20 mai 2015, les députés européens avaient pourtant voté en session plénière une résolution demandant la reprise des négociations, et appelant la Commission européenne à ne pas retirer son projet de directive, ou à présenter une nouvelle initiative législative avant la fin de l'année. Jusqu'à présent, les retraits générés par l'opposition du Conseil étaient généralement précédés des négociations interinstitutionnelles. La Commission Juncker semble s'être affranchie de ces contraintes en procédant à des retraits systématiques concernant des propositions dont le Parlement souhaitait poursuivre l'examen. Si l'abandon de sa proposition par la Commission s'inscrit incontestablement dans le cadre du « Mieux légiférer », cette dernière a déployé beaucoup d'efforts pour parvenir à l'effectivité de la révision. Mais ne doit-on pas attendre de la Commission la recherche d'un consensus quelles que soient les difficultés?

La représentation des femmes dans les conseils des sociétés. Pour favoriser la parité entre les hommes et les femmes, la Commission européenne a présenté, le 14 novembre 2012, une proposition de directive destinée à assurer une représentation plus équilibrée des hommes et des femmes dans les conseils d'administration et de surveillance des sociétés cotées en bourse ${ }^{6}$, à l'exclusion des $\mathrm{PME}^{7}$. Plus précisément, la proposition de directive avait pour objectif d'accroître sensiblement, dans l'ensemble de l'Union, le nombre de femmes dans les organes de décision économique, en fixant un objectif minimum de $40 \%$ de femmes parmi les administrateurs non exécutifs des sociétés cotées à l'horizon 2020 , et 2018 pour les entreprises publiques sur lesquelles les pouvoirs publics exercent une influence dominante. À titre de mesure complémentaire, la proposition imposait également aux mêmes sociétés, dans le cadre de l'autorégulation, de se fixer des objectifs concernant la représentation des deux sexes

\footnotetext{
3 Directive du 19 octobre 1992 « concernant la mise en œuvre de mesures visant à promouvoir l'amélioration de la sécurité et de la santé des travailleuses enceintes, accouchées ou allaitantes au travail ».

4 Proposition de directive du Parlement européen et du Conseil portant modification de la directive 92/85/CEE du Conseil concernant la mise en œuvre de mesures visant à promouvoir l'amélioration de la sécurité et de la santé des travailleuses enceintes, accouchées ou allaitantes au travail, 3 octobre 2008 , COM (2008) 637 final.

5 Ce qui fut fait avec l'initiative sur l'équilibre entre vie professionnelle et vie familiale (cf. infra), laquelle ne règle pas, cependant, la question de l'allongement du congé de maternité.

$6 \quad$ Proposition de directive du Parlement européen et du Conseil relative à un meilleur équilibre hommes-femmes parmi les administrateurs non exécutifs des sociétés cotées en bourse et à des mesures connexes, COM(2012) 614 final. Sur la proposition, cf. note J. REDENIUS-HOEVERMANN, « Améliorer l'équilibre hommes/femmes parmi les administrateurs des sociétés cotées en bourse? », fournal de droit européen, 2013 , p. 95.

$7 \quad$ Telles que définies par la recommandation 2003/361 de la Commission.
} 
parmi leurs administrateurs exécutifs, cet objectif qualifié de « souple » par la Commission ellemême étant à atteindre au plus tard en 2020, et 2018 pour les entreprises publiques.

Le projet, voté par le Parlement le 20 novembre 2013, a été débattu trois fois au Conseil sans qu'un consensus ait pu être dégagé. La Commission a affirmé en 2015 continuer à soutenir l'adoption de la proposition de directive ${ }^{8}$, l'ayant, au demeurant, inscrite dans la liste des propositions prioritaires en attente dans le programme de travail pour 2016. Toutefois, la proposition a disparu de cette liste dans le programme de travail pour 2017. Le projet a été abandonné au nom de l'allégement de la législation de l'Union, mais cette proposition a suscité un réel blocage au Conseil, certains États membres s'y opposant au nom de la subsidiarité.

La révision de la directive 2003/88 sur l'aménagement du temps de travail, engagée par la Commission européenne en $2004^{9}$, a été abandonnée, cette révision s'avérant, il est vrai, difficile. Il s'agissait, notamment, de préciser les règles relatives aux temps de garde dans certains secteurs d'activité suite à la jurisprudence de la Cour de justice, de prévoir davantage de flexibilité pour le calcul de la durée maximale de travail du temps de travail hebdomadaire, et de réexaminer l'" optout » individuel. Les négociations entre les États au sein du Conseil, puis entre le Parlement européen et le Conseil, ont duré cinq ans, pour aboutir finalement à un échec en 2009. Le Parlement a refusé la clause dite d' «opt-out », préconisé l'assimilation du temps de garde à du temps de travail, ainsi que le calcul du temps de travail par travailleur et non par contrat pour les travailleurs disposant de contrats multiples. Suite à l'échec du trilogue, la Commission fit une seconde tentative pour parvenir à la révision de la directive. De nouvelles négociations entre les partenaires sociaux ont été entamées en 2010, prolongées jusqu'à fin 2012, mais ces derniers n'ont pas réussi à trouver un terrain d'entente, notamment à propos de la clause d' «opt-out», ainsi que sur le temps de garde et le repos compensatoire. La Commission a ouvert une consultation publique qui s'est déroulée du $1^{\text {er }}$ décembre 2014 au 18 mars 2015 sur la révision de la directive, annoncé une nouvelle proposition pour 2016, pour finalement abandonner son projet de réforme au profit d'un guide destiné à améliorer l'application de la directive existante, comme annoncé dans son programme de travail pour l'année $2017^{10}$. Avec pour objectif d'améliorer la sécurité et la clarté juridiques, mais dénuée de tout caractère contraignant, la « communication interprétative » de clarification fournit une exégèse de la directive, soit en reprenant la jurisprudence de la Cour de justice, soit en donnant la propre interprétation de la Commission lorsque la jurisprudence est absente ou peu probante. Cette initiative, conçue comme une réponse au volume croissant du contentieux, a pour vocation de permettre une mise en œuvre pertinente et d'éviter les infractions. Elle s'inscrit sans aucun doute dans la perspective de la Communication du 19 janvier 2017 relative au « Mieux légiférer », mais traduit également la grande difficulté à réaliser la révision de la directive de 2003.

\footnotetext{
8 Commission européenne, Engagement stratégique pour l'égalité entre les femmes et les hommes 2016-2019 Programme de travail pour 2016, annexe III, pt 3.

9 COM (2004) 607 final.

10 Communication interprétative relative à la directive 2003/88 du Parlement européen et du Conseil du 4 novembre 2003 concernant certains aspects de l'aménagement du temps de travail, 2017/C167/01, fOUE 24 mai 2017, C165/01.
} 
La proposition de directive horizontale sur l'égalité de traitement. Devant marquer une étape importante dans la lutte contre les discriminations et destinée à compléter le dispositif existant, une proposition de directive horizontale relative à l'égalité de traitement entre les personnes sans distinction de religion ou de conviction, de handicap, d'âge ou d'orientation sexuelle ${ }^{11}$ a été déposée le 2 juillet 2008. L'objectif était de mettre en œuvre le principe de l'égalité de traitement dans les domaines de la protection sociale, y compris la sécurité sociale, des soins de santé, des avantages sociaux, mais également de l'éducation, de l'accès aux biens et aux services, et de la fourniture de biens et services à la disposition du public y compris en matière de logement. Pour ce faire, la proposition préconisait d'interdire les discriminations tant directes qu'indirectes, ainsi que le harcèlement et les rétorsions. Bloquée au Conseil depuis que le Parlement a arrêté sa position le 2 avril $2009^{12}$ soutenant en substance la proposition de la Commission, reportée plusieurs fois, n'étant plus soutenue que par un petit nombre d'États, la proposition est tombée dans les oubliettes, la Commission n'envisageant pas pour l'heure une nouvelle proposition.

\subsection{La multiplication des analyses d'impact}

Aux termes de la nouvelle politique du « Mieux légiférer », l'amélioration de la législation nécessite le recours à une expertise probante, concrétisée par un accroissement significatif des analyses d'impact. S'en trouvent affectés tant les co-législateurs que les partenaires sociaux.

Le législateur sous la férule des experts. Selon l'accord interinstitutionnel du 13 avril 2016, une analyse d'impact sera effectuée chaque fois que l'initiative de la Commission, qu'elle soit législative ou non, sera susceptible d'avoir une incidence économique, environnementale ou sociale importante. En outre, les initiatives prévues dans le programme de travail de la Commission feront, en principe, l'objet d'une analyse d'impact. On peut donc en déduire que toute initiative sociale sera accompagnée d'une analyse d'impact. Il est vrai que cette approche traduit la pratique déjà bien ancrée d'un recours de plus en plus en plus fréquent aux analyses d'impact de la part de la Commission, notamment en la matière. La communication de la Commission du 19 mai 2015 a ajouté de nouveaux critères à la boîte à outils pour l'évaluation des initiatives dans le domaine social ${ }^{13}$ qui laissent certains observateurs perplexes ${ }^{14}$. Par ailleurs, l'efficacité de l'instrument s'avère conditionnée par la méthodologie qui sera utilisée autant que par sa qualité.

Ledit accord prévoit également que Parlement et Conseil sont tenus de tenir « pleinement compte » des analyses d'impact de la Commission lors de l'examen des propositions législatives, ce qui restreint sans conteste la possibilité de modifier les propositions de la Commission alors que le

11 Proposition de directive du Conseil relative à la mise en œuvre du principe de l'égalité de traitement entre les personnes sans distinction de religion ou de convictions, de handicap, d'âge ou d'orientation sexuelle, 2 juillet 2008, COM (2008) 0426.

12 FOUE C 137 E, 27 mai 2010, p. 68.

Better Regulation « toolbox », outils 7 et 25, Commission staff working document, Better Regulation Guidelines, SWD (2015) 111final.

E. VAN Den AbeELe, Le pilier social de l'UE à l'épreuve du mieux légiférer Étude CESE, novembre 2016, p.6. 
Parlement européen se montre enclin à améliorer ces dernières. En revanche, le détricotage des propositions de la Commission par le Conseil pourrait s'en trouver gêné.

Par ailleurs, la Commission souhaite que l'utilisation des analyses d'impact soit étendue au Parlement et au Conseil, ces deux institutions étant appelées à effectuer une analyse d'impact s'ils modifient de manière significative les propositions de la Commission. Outre qu'il reste à déterminer ce qu'il convient d'entendre par «modification substantielle », ces institutions, demeurant peu enclines aujourd'hui à recourir à de telles analyses, disposent-elles des ressources matérielles et humaines suffisantes ? Une telle pratique rendra de toute évidence les modifications des propositions de la Commission plus compliquées et plus hasardeuses, bridant ainsi la force de proposition du Parlement.

Pus globalement et ainsi que l'observe la Confédération européenne des syndicats, la généralisation des analyses d'impact « introduit une procédure plus longue, plus onéreuse et plus bureaucratique qui risque de freiner le progrès social $»^{15}$.

L'efficience du dialogue social en question. Le dialogue social est également soumis aux analyses d'impact, ce qui porte notamment atteinte à l'autonomie des partenaires sociaux, à propos de la mise en ouvre d'un accord par voie de directive.

Aux termes de l'article $155 \S 2$ du TFUE, les partenaires sociaux peuvent conjointement demander à la Commission de présenter une proposition de « décision du Conseil », en pratique une directive ${ }^{16}$, visant à assurer la mise en œuvre de leurs accords. Or, le droit d'initiative de la Commission lui confère le pouvoir d'apprécier l'opportunité de saisir le Conseil ${ }^{17}$, sans toutefois posséder la faculté de modifier le contenu de l'accord. La Commission a usé de ce pouvoir dans l'esprit du « Mieux légiférer » à propos du premier accord-cadre signé dans la coiffure.

Désormais, à l'instar des principes énoncés par la Commission afin de mieux légiférer, la décision de saisir le Conseil sera étayée par une analyse d'impact proportionnée dès lors que l'accord en cause sera susceptible d'avoir des incidences significatives. Celle-ci mettra l'accent en particulier sur la représentativité des parties signataires, la légalité de l'accord par rapport au cadre juridique de l'Union et le respect des principes de subsidiarité et de proportionnalité. Du fait du rôle et de l'autonomie des partenaires sociaux, les analyses d'impact devront, en l'occurrence, être accompagnées de modalités spécifiques qu'il reste à préciser.

Dans la mesure où le droit même de la Commission de juger de l'opportunité de saisir le Conseil n'est pas à l'abri de la critique, contesté au demeurant par les partenaires sociaux, le préalable de l'analyse d'impact ne peut, a fortiori, emporter l'adhésion.

Confédération européenne des syndicats, déclaration de la CES sur le paquet « Mieux légiférer ».

Instrument choisi par la Commission.

Ce qui fut confirmé par le TPICE, 17 juin 1998, UEAPME, aff. T-135/96, Rec. II, p. 2335. 


\section{2) Les avancées sociales dans le respect du «Mieux légiférer»}

Si le socle des droits sociaux récemment adopté marque incontestablement une évolution positive, celui-ci ne confère, néanmoins, aucun droit nouveau aux travailleurs et assurés sociaux. Au-delà, les quelques initiatives présentées n'offrent aucun caractère novateur et cette réticence à promouvoir de nouvelles normes est sans aucun doute marquée du sceau du « Mieux légiférer ».

\subsection{L'élaboration d'un socle européen des droits sociaux et les initiatives subséquentes}

Après une première ébauche élaborée en mars 2016, suivie d'une vaste consultation publique entre mai et décembre 2016, la Commission a présenté une proposition, sous forme de recommandation, portant sur un socle des droits sociaux ${ }^{18}$. Ce dernier repose sur 20 principes et droits essentiels s'articulant autour de trois objectifs : l'égalité des chances et l'accès au marché du travail pour tous ; l'équité dans les conditions de travail ; une protection sociale adéquate favorisant l'inclusion. Avec pour dessein de favoriser la convergence des droits nationaux par le haut, il est conçu prioritairement pour la zone euro du fait de l'opposition de certains États membres tels que la Pologne ou la Hongrie à toute avancée sociale, mais concerne potentiellement tous les États membres souhaitant y adhérer.

Même si l'initiative mérite d'être saluée dans la mesure où elle succède à de nombreuses années d'immobilisme, force est de constater qu'il s'agit d'une avancée modérée qui porte la marque REFIT. Dépourvu de force contraignante, le socle européen des droits sociaux n'est que l'énoncé d'un certain nombre de principes. Fondé sur l'acquis social déjà existant, sa finalité n'est pas clairement définie. Ne s'agit-il pas essentiellement d'une d'incitation à la convergence des règles nationales, autrement dit d'un cadre destiné à encourager les États membres à modifier eux-mêmes leurs propres législations? En outre, la mise en œuvre de ce socle n'est assortie d'aucun engagement. À l'occasion de la publication de ce dernier, Maryanne Thyssen a d'ailleurs affirmé qu'il était hors de question d'introduire trop de normes.

Dans la continuité du socle des droits sociaux et simultanément, mais aussi conformément à son programme de travail pour 2017, la Commission a présenté plusieurs initiatives, outre la communication portant interprétation de la directive relative à l'aménagement du temps de travail, et qui pour reprendre les termes mêmes de la Commission, n'en constitue qu'une clarification ${ }^{19}$ et sert de palliatif à une réforme abandonnée.

En premier lieu fut déposée une initiative visant à promouvoir l'équilibre entre vie professionnelle et vie privée des parents et des aidants qui travaillent ${ }^{20}$. Cette initiative fait suite au retrait,

\footnotetext{
18 Recommandation 2017/761 sur le socle européen des droits sociaux, FOUE 29 avril 2017, L113/56.

19 cf. supra.

Communication de la Commission, Initiative visant à promouvoir l'équilibre entre vie professionnelle et vie privée des parents et aidants qui travaillent, 26 avril 2017, COM (2017) 252 final
} 
en 2015, de la proposition de la Commission relative à la révision de la directive 92/85/CEE sur la protection de la maternité ${ }^{21}$. S'inscrivant dans la perspective de l'égalité des sexes, elle comporte un ensemble de mesures dont la plupart sont de nature non législative, s'inscrivant en cela dans la perspective du « Mieux légiférer ». Elle renferme, néanmoins, une proposition de directive qui tend à préserver et développer les droits existants, notamment ceux garantis par la directive sur le congé parental. Elle propose ainsi la généralisation d'un congé de paternité de dix jours à l'occasion de la naissance d'un enfant, un congé parental de quatre mois jusqu'aux douze ans de l'enfant rémunéré à hauteur des indemnités de maladie, un congé pour s'occuper de proches gravement malades ou dépendants à hauteur de cinq jours par an rémunéré dans les mêmes conditions, le droit des parents d'enfants âgés au maximum de douze ans et des travailleurs qui s'occupent de proches dépendants à demander des formules de travail souples, assorti d'une protection contre le licenciement de ceux qui en font la demande.

La Commission a par ailleurs lancé deux consultations des partenaires sociaux conformément à l'article 154 TFUE, l'une sur la révision de la directive 91/533 relative à l'information du travailleur sur ses conditions de travail ${ }^{22}$ dans une perspective de modernisation et au titre du programme REFIT, et l'autre sur une action en faveur de l'accès de tous les citoyens à la protection sociale et aux services de l'emploi.

\subsection{La rareté des nouvelles initiatives législatives}

En dehors des initiatives précédemment évoquées et présentées dans la continuité du contexte du socle des droits sociaux, l'heure est la disette en matière sociale, et il faut y voir incontestablement la marque du « Mieux légiférer ». Aucune initiative novatrice, ambitieuse, n'est envisagée et certains textes pourtant attendus ne sont pas d'actualité.

En matière de santé et de sécurité, aucune nouvelle proposition de directive n'est annoncée dans le programme de travail pour l'année 2017, lequel se situe dans la ligne du cadre stratégique pour la période 2014-2020 et s'avère incontestablement marqué de la volonté d'une réduction des coûts pour les entreprises.

Dans une communication en date du 10 janvier $2017^{23}$, la Commission confirme la nécessité d'encourager les États membres à revoir leur législation en la matière. Si les normes nationales sont plus détaillées que les prescriptions de l'Union européenne encore faut-il que la protection des travailleurs s'en trouve renforcée, mais également « que les entreprises ne soient soumises à aucune charge administrative supplémentaire inutile».

21 Cf. supra.

22 Document de consultation du 26 avril 2017, Première phase d'une consultation des partenaires sociaux, organisée en vertu de l'article 154 TFUE sur l'éventualité d'une révision de la directive relative à la déclaration écrite (directive 91/533/CEE) dans le cadre du socle des droits sociaux C (2017), 2611final.

23 Des conditions de travail plus sûres et plus saines pour tous - Moderniser la législation et la politique de l'Union européenne en matière de sécurité et de santé au travail, COM (2017) 12 final. 
Par ailleurs, il est envisagé de supprimer ou d'actualiser les dispositions caduques d'un certain nombre de directives, six d'entre elles ayant été pointées comme prioritaires, avec pour finalité clarté, cohérence et pertinence des normes. Il s'agit, certes, de simplifier et de réduire la charge administrative pour les entreprises et les autorités de contrôle, mais dans le maintien voire l'amélioration de la protection des travailleurs.

En revanche, le déblocage d'une proposition de directive datant déjà de plusieurs années et relative aux troubles musculo-squelettiques a été sacrifié sur l'autel du programme REFIT par le mandat précédent de la Commission européenne ${ }^{24}$ et n'a pas été exhumé. Quant à la proposition de directive sur les risques psychosociaux pourtant réclamée et un temps attendu, il n'en n'est plus question.

Seule la révision de la directive sur les agents cancérigènes et mutagènes ${ }^{25}$ sur le métier depuis plus de dix ans, a suivi son cours, même si la Commission avait annoncé son abandon dans le programme REFIT. En mai 2016, donc au moment du dernier paquet « Mieux légiférer », la Commission a proposé d'ajouter de nouvelles valeurs limites ou de modifier des valeurs existantes pour réduire l'exposition à de nouveaux agents cancérigènes ${ }^{26}$ sur les lieux du travail ${ }^{27}$. Si le dossier a été longtemps bloqué, le Conseil et le Parlement sont parvenus à un accord le 11 juillet 2017 qui va bien au-delà de la proposition de la Commission européenne laquelle se limitait à la question des valeurs limites d'exposition. Les co-législateurs ont dépassé la frilosité de cette dernière, en lui imposant, notamment, d'envisager la possibilité d'inclure les substances reprotoxiques dans le champ d'application de la directive. Par ailleurs, une seconde proposition de directive datant de début janvier 2017 est à l'heure actuelle sur le bureau du Parlement européen, et une troisième révision est annoncée pour début 2018.

En revanche, la mise en œuvre par voie de directive, demandée par les partenaires sociaux, de l'Accord-cadre européen d'avril 2012 sur la protection de la santé et de la sécurité au travail dans le secteur de la coiffure aurait en son temps été bienvenue. Malgré l'opposition de dix États membres affirmée avant même la moindre initiative de la part de la Commission, cette dernière avait indiqué en 2014, en réponse à une question parlementaire, qu'une étude externe coûts/avantages avait été finalisée et qu'elle était en train de rédiger sa propre appréciation comme préalable à toute décision, pour finalement renoncer à la directive attendue. Cette position avait, au demeurant, été annoncée dans le programme REFIT. Un nouvel accord-cadre a été conclu le 23 juin 2016 après un long travail de réécriture, en lien avec la Commission et le Conseil, et les partenaires sociaux ont demandé, une nouvelle fois, qu'il soit mis en œuvre par voie de directive. Cependant, la Commission procédera, à une analyse d'impact dotée des effets prévus par l'accord interinstitutionnel relatif à l'amélioration de la réglementation du 13 avril $2016^{28}$. Si elle est favorable au texte, elle invitera le Conseil à adopter la directive sollicitée.

\footnotetext{
24 COM (2013) 685 final.

25 Directive 2004/37/CE

26 La directive 2004/37/CE n'en vise que trois.

27 Proposition de directive du Parlement européen et du Conseil modifiant la directive 2004/37/CE concernant la protection des travailleurs contre les risques liés à l'exposition à des agents cancérigènes ou mutagènes au travail, 13 mai 2016, COM (2016) 248 final.

28 Aux termes de cet accord, le Parlement européen et le Conseil tiennent pleinement compte des analyses d'impact de la Commission lorsqu'ils se prononcent sur les propositions législatives.
} 
L'information et la consultation des travailleurs. Dans une résolution en date du 15 janvier 2013 - également appelée rapport Cercas - le Parlement européen, soutenu par la CES, avait demandé à la Commission, au titre de l'article 225 TFUE, de présenter une proposition d' " acte législatif » sur l'information et la consultation des travailleurs, l'anticipation et la gestion des restructurations. Dans une communication du 13 décembre $2013^{29}$, la Commission répond que dans le cadre de sa politique visant à mieux légiférer, elle a dressé un « bilan de qualité » du droit de l'Union en matière d'implication des travailleurs. Selon ce bilan, le cadre législatif est globalement adapté aux besoins : dans l'ensemble, les trois directives sont pertinentes, efficaces et cohérentes, et se renforcent mutuellement ; de même, les avantages qu'elles génèrent sont probablement supérieurs à leurs coûts. L'évaluation a, toutefois, mis en lumière des lacunes et des faiblesses concernant le champ d'application et la mise en ouvre de ces directives. Certains acteurs ont remis en question le fait qu'elles ne s'appliquaient pas, notamment, aux petites entreprises ni aux administrations publiques jugeant que cette exclusion limitait l'utilité pratique des directives pour une part importante des travailleurs.

Le bilan de qualité a également pointé quelques facteurs qui, en certaines circonstances, ont peut-être contribué à réduire l'efficacité des directives. Il s'agit, notamment, du petit nombre d'organes représentatifs, de la qualité de leur implication compte tenu d'un mode de consultation souvent limité ou purement formel, de leur influence stratégique, de la méconnaissance des droits et obligations, ainsi que du respect et du contrôle de l'application des textes. En conséquence, certains objectifs des directives ne semblent pas avoir été pleinement réalisés, notamment la réduction du nombre de licenciements collectifs, l'amélioration de la gestion et de l'anticipation des changements, ainsi que le renforcement de la capacité d'adaptation et d'insertion professionnelle des travailleurs. Enfin, le bilan a mis en évidence de possibles incohérences entre les différentes directives traitant de l'information et de la consultation des travailleurs, quant à certaines définitions. La suppression de ces insuffisances contribuerait aussi à améliorer les processus de restructuration des entreprises.

Depuis quelques années déjà, la Commission s'est engagée à étudier la possibilité de consolider les directives sur l'information et la consultation ${ }^{30}$, volonté confirmée dans la communication sur une réglementation affûtée et performante adoptée le 2 octobre $2013^{31}$. Début 2015, fut lancée la première phase de consultation des partenaires sociaux sur la possibilité de fusionner les trois directives concernées en un seul texte législatif, une meilleure articulation possible des notions d'information et de consultation et enfin sur l'inclusion de l'administration publique dans le champ d'application des directives concernées. Un accord a été conclu entre les partenaires sociaux sur ce dernier point en décembre 2015, lesquels ont demandé la mise en œuvre de l'accord par voie de directive. Conformément aux principes dégagés dans le cadre de la nouvelle politique du « Mieux légiférer », l'élaboration d'une analyse d'impact de l'accord est sur le métier. Ce n'est qu'après avoir pris sa décision sur l'accord que la Commission établira s'il convient de procéder à la seconde phase de consultation des partenaires sociaux.

29 Communication présentant un « cadre de qualité de l'Union européenne pour l'anticipation des changements et des restructurations », COM (2013) 882 final.

30 Directive 98/59/CE concernant les licenciements collectifs ; Directive 2001/23/CE concernant les transferts d'entreprises ; Directive 2002/14/CE établissant un cadre général relatif à l'information et la consultation des travailleurs.

31 COM (2013) 685 final 
La révision de la directive relative au détachement des travailleurs. Exception notable à cet immobilisme en matière sociale, la révision de la directive 96/71/CE relative au détachement des travailleurs dans le cadre d'une prestation de services, prévue dans le programme de travail de la Commission et présentée contre vents et marées en mars $2016^{32}$. Cette révision a pour but d'encadrer plus strictement le recours aux travailleurs détachés afin de lutter contre les pratiques déloyales, et d'assurer à ces derniers l'égalité de traitement en matière salariale. Bien que les parlements nationaux de onze États membres aient déclenché la procédure dite du « carton jaune » - à savoir le mécanisme de contrôle de la subsidiarité institué par le traité de Lisbonne ${ }^{33}$ - la Commission a maintenu sa proposition et a mis tout en œuvre afin de parvenir à un accord. Après plus d'un an de vives discussions, un compromis a été trouvé au Conseil du 23 octobre 2017 au prix de concessions importantes. Une avancée, certes, mais en demi-teinte. La durée du détachement a été fixée à douze mois avec une possibilité d'extension à dix-huit mois, et l'égalité de rémunération entre travailleurs détachés et travailleurs locaux a été actée. En revanche, les transports routiers ont été exclus du champ d'application des nouvelles normes alors que l'application de l'ensemble des accords collectifs n'a pas été prévue. Enfin, le délai de transposition de la directive révisée pourra aller jusqu'à quatre ans, au lieu du délai habituel de deux ans.

La matière est sensible et cette révision, envisagée depuis déjà quelques années puis écartée dans un premier temps au profit d'une directive destinée a assurer la bonne application des normes existantes, a été demandée par quelques États avec insistance que le « Mieux légiférer » n'était pas de nature à affecter.

Il est indéniable que la nouvelle politique du « Mieux légiférer » autant que le programme REFIT interfèrent avec la progression des normes sociales. Ceux-ci ont conduit à l'abandon de propositions significatives, alourdissent le processus décisionnel, et confient les avancées sociales aux mains des experts au détriment des co-législateurs et des partenaires sociaux. En dépit de l'appel du «triple A social » pour l'Union européenne de la part de Jean-Claude Junker, le renforcement des droits des travailleurs, déjà malaisé du fait des réticences de la part de certains États membres, sera sans conteste plus difficile ou à tout le moins ralenti, plus lent que ne l'exige l'attente des citoyens.

COM (2016)128 final.

33 Article $5 \S 2$ al.2 et article 12 point $\mathrm{b}$ ). 


\section{Éléments bibliographiques}

A. Alemano, « Dans quelle mesure le « Mieux légiférer » est-il meilleur ? », RDUE, 4/2015, p. 509-526.

Confédération européenne des syndicats, déclaration de la CES sur le paquet « Mieux légiférer », https://www.etuc.org.

Confédération européenne des syndicats, Mieux légiférer ou moins de démocratie et plus de bureaucratie ?, https://www.etuc.org.

Confédération européenne des syndicats, Proposition d'accord interinstitutionnel relatif à l'amélioration de la réglementation, Document de position de la CES, https://www.etuc.org.

K. LÖRCHER and I. SCHÖMANN, The European pillar of social rights: critical legal analysis and proposals, 2016, ETUI, Brussels.

Ch. De Marcilly, M. Touillon, «Programme « Mieux légiférer»: l'expertise surpasse-t-elle le politique ?», Fondation Robert Schuman, Questions d'Europe n³70, 9 novembre 2015.

M. Prokopiak, « Un nouvel accord interinstitutionnel pour « Mieux légiférer » », fournal d'actualité des droits européens, avril 2016, http://revue-jade.eu/article/view/1600.

M.-J. RodRigues, Rapport sur un socle européen de droits sociaux, Parlement européen, Document 2016/2095, 20 décembre 2016.

I. SchömanN, « Mieux légiférer dans l'Union Européenne : Simplifier ou dénaturer l'acquis communautaire ? Analyse critique d'une initiative de la Commission au regard du Droit du travail », Revue de droit comparé du travail et de la sécurité sociale, 2016/1, p. 6.

C-E. Triomphe, I. Schömann, 28 novembre 2015, Droit social européen : dessous alarmants de la simplification $1^{\text {re }}$ partie, Métis,http://www.metiseurope.eu/ droit-social-europeen-dessous-alarmants-de-la-simplification-1.

C-E. Triomphe, I. Schömann, Droit social européen : dessous alarmants de la simplification $2^{\mathrm{e}}$ partie, 9 décembre 2015, Métis, http://www.metiseurope.eu/droit-social-europeen-dessous-alarmants-de-la-simplification-2.

E. VAN Den AbeELE, Le pilier social de l'UE à l'épreuve du «Mieux légiférer», Étude CESE, novembre 2016.

E. VAN Den AbeELE, « Mieux légiférer » : une simplification bureaucratique à visée politique », ETUI, working paper 2015.04.

L. Vogel et E. VAN Den Abeele, Better regulation : perspectives critiques, Rapport 113, ETUI. 


\title{
Ambitions et insuffisances légistiques EN MATIÈ RE DE MIGRATION LÉGALE L'APPLICATION DE LA STRATÉGIE « MIEUX LÉGIFÉRER » À LA DIRECTIVE « CARTE BLEUE»
}

\author{
Romain FOUCART ${ }^{\mathbf{1}}$
}

\section{Résumé}

Le processus de révision de la directive « Carte bleue », relative à l'immigration hautement qualifiée dans l'UE, met en lumière les ambitions et les limites de la transposition du « Mieux légiférer » au domaine de l'immigration. La méthode retenue et les moyens alloués interrogent sur l'existence d'une réelle volonté d'améliorer la législation dans une matière particulièrement sensible sur le plan politique.

\section{Abstract}

The current legislative recast of the "blue card" directive on highly skilled migration to the European Union shows the ambitions and limits of the «Better regulation » agenda applied in migration issues. The method chosen as well as the resources allocated question the existence of a proper will to improve the EU legislative acquis in such a politically sensitive matter.

Conclu en mai 2016, l'accord interinstitutionnel « Mieux légiférer » prévoit à son article 2 que les trois institutions principales de l'Union européenne promeuvent « la simplicité, la clarté et la cohérence dans la rédaction de la législation [...], ainsi qu'une plus grande transparence du processus législatif ${ }^{2}$. Cet accord s'inscrit dans le cadre d'une stratégie poursuivie par la Commission européenne depuis $2000^{3}$, destinée à répondre aux objectifs du protocole sur la bonne gouvernance annexé au Traité lors de la réforme d'Amsterdam. Pour reprendre la formule du professeur Jean-Paul Jacqué, mieux légiférer a, depuis Jacques Delors, « été une préoccupation constante des présidents successifs de la Commission » ${ }^{4}$.

Doctorant, Aix Marseille Univ, Université de Toulon, Univ Pau \& Pays Adour, CNRS, DICE, CERIC, Aix-en-Provence, France.

Accord interinstitutionnel entre le Parlement européen, le Conseil de l'Union européenne et la Commission européenne « Mieux légiférer » du 13 avril 2016, FOCE L 123 du 12.05.2016, p.1, pt 2.

3 Notamment depuis les conclusions du Conseil européen de Lisbonne de mars 2000.

$4 \quad$ J.-P. JacouÉ, « Mieux légiférer », RTDE, 2015, p. 281. 
Apparu outre-Atlantique, c'est au Royaume-Uni que cet impératif fait son entrée sur le continent européen dès le milieu des années $1980^{5}$. Son appropriation par les Communautés européennes opérera à partir du début de la décennie 1990, notamment à la suite des conclusions du Conseil européen d'Édimbourg. À cette occasion, les chefs d'État ou de gouvernement demandent à la Commission d'adopter des «mesures concrètes pour améliorer la qualité de la législation », en vue de « rendre la nouvelle législation communautaire plus claire et plus simple $»^{6}$. Cette requête intervient après le développement exponentiel du volume de la législation communautaire au tournant des années 1990, qui accompagne l'achèvement du marché unique et l'accroissement continu des compétences des Communautés.

Cet accroissement s'illustre particulièrement par la construction de la structure en piliers du Traité de Maastricht, qui permet aux États membres d'appréhender de manière conjointe l'immigration des ressortissants de pays tiers. Aux termes de l'article K1 du Traité sur l'Union européenne (TUE) d'alors, l'immigration constitue une question d'intérêt commun partagée par les États membres, au même titre que les coopérations policière ou judiciaire, versée au sein du troisième pilier. Cette avancée fondamentale ne doit toutefois pas être surestimée, dans la mesure où elle exclut à l'origine les institutions communautaires de ces matières. Suite à l'incorporation de l'immigration dans le pilier communautaire opérée par la réforme d'Amsterdam, ces dernières devront encore attendre l'expiration d'une période transitoire de cinq ans avant de pouvoir user de leur compétence législative. Jusqu'à cette date, le Conseil demeure la seule institution capable de légiférer sur le sujet. L'institution représentant les intérêts des États s'assurera que les discussions à venir prennent comme point de référence les instruments adoptés avant la fin de la période transitoire, sous l'empire de la méthode intergouvernementale. Il faudra attendre la fin de la structure en piliers résultant de la réforme de Lisbonne de 2009 pour impliquer davantage les institutions communautaires dans le développement de la politique migratoire de l'Union, sans aller jusqu'à effacer la logique qui sous-tend l'adoption des instruments législatifs dans ce domaine particulièrement clivant.

Le choix de la méthode privilégiée dans ce domaine législatif, qui se caractérise par une approche segmentée et non transversale des problématiques posées, s'explique par différents facteurs. Il convient en effet de rappeler l'échec des propositions législatives transversales formulées par la Commission lors des premières années de la politique d'immigration de l'Union? ${ }^{7}$. Ces revers ont amené la Commission à revoir ses ambitions à la baisse en évitant autant que possible de défendre des textes transversaux susceptibles de susciter la méfiance des États membres, pour qui l'admission de ressortissants de pays tiers demeure un attribut essentiel de la souveraineté. La logique initiale

\footnotetext{
5 M. HADJIISKY, « La better regulation entre coordination européenne et relations triangulaires : OCDE, États membres, Union européenne », RFAP, 2016/2, n 158, p. 561-575, spéc. p.570.

Conseil européen, Conclusions de la présidence, 11 et 12 décembre 1992, Annexe 3 à la partie A, p. 4-7.

Commission européenne, Proposition de directive du Conseil relative aux conditions d'entrée et de séjour des ressortissants de pays tiers aux fins d'un emploi salarié ou de l'exercice d'une activité économique indépendante, COM (2001) 386 final, adoptée le 11 juillet 2001 et retirée le 17 mars 2006 devant les refus répétés d'examen au Conseil. Voir aussi dans le même ordre d'idées la proposition de méthode ouverte coordination de la politique communautaire, qui restera elle aussi lettre morte : Commission européenne, Communication de la Commission au Conseil et au Parlement européen sur une méthode ouverte de coordination de la politique communautaire en matière d'immigration, COM (2001) 387 final, 12 juillet 2001.
} 
a fait place à une approche ciblée, qui vise à réguler l'admission de catégories précises de ressortissants de pays tiers. Ce basculement se manifeste entre autres par l'adoption de textes relatifs à l'admission des chercheurs, des étudiants, des travailleurs saisonniers, au regroupement familial ou au séjour des résidents de longue durée ${ }^{8}$. Malgré ce changement stratégique, la réticence des États membres demeure. L'admission de ressortissants de pays tiers constitue une attribution régalienne que ces derniers ne sont pas prêts à céder automatiquement à l'Union, en dépit de la compétence législative en la matière accordée par le Traité. Cette réserve s'exprime avec une récurrence incontestable à chaque étape du processus décisionnel. Elle s'illustre par l'atténuation systématique des propositions de la Commission par le Conseil avant adoption définitive, et se concrétise en pratique par des textes adoptés sur la base du plus petit dénominateur commun.

Ces difficultés emportent plusieurs conséquences négatives. Les outils adoptés se caractérisent tout d'abord par leur émiettement, leur chevauchement et leur manque de cohérence, qui sont les conséquences de la stratégie législative privilégiée depuis le milieu de la décennie 2000. Cet obstacle se conjugue au manque d'efficacité et à la multiplication de dispositions dérogatoires à l'intérieur même d'instruments pourtant supposés participer à la mise en œuvre d'une politique commune. La base de cet écueil supplémentaire se trouve dans la réserve qu'expriment les États membres lorsqu'il s'agit d'accorder des concessions dans le processus législatif. Cette réticence trouve, de plus, un argument de poids dans les dispositions mêmes du Traité qui fondent la compétence de l'Union en matière d'immigration. Le paragraphe 5 de l'article 79 TFUE précise en effet que les États membres demeurent compétents pour fixer le volume d'admission de ressortissants de pays tiers sur leur territoire à des fins de travail'.

La critique du manque d'efficacité de la politique de migration légale de l'Union apparaît dans les faits aussi ancienne que la politique elle-même. Depuis la création d'une politique de l'Union dédiée à l'immigration de ressortissants de pays tiers par le Traité d'Amsterdam, il n'est de projet législatif qui ne soit critiqué pour son absence de vision ou son caractère au mieux superflu, au pire inefficace $^{10}$.

\footnotetext{
8 Directive 2005/71/CE du Conseil du 12 octobre 2005 relative à une procédure d'admission spécifique des ressortissants de pays tiers aux fins de recherche scientifique, JOCE L 289 du 3.11.2005, p. 15-22 ; Directive 2004/114/CE du Conseil du 13 décembre 2004 relative aux conditions d'admission des ressortissants de pays tiers à des fins d'études, d'échange d'élèves, de formation non rémunérée ou de volontariat ; $7 O C E$ L 375 du 23.12.2004, p. 12-18 ; Directive 2014/36/UE du Parlement européen et du Conseil du 26 février 2014 établissant les conditions d'entrée et de séjour des ressortissants de pays tiers aux fins d'un emploi en tant que travailleur saisonnier, fOUE L 94 du 28.3.2014, p. 375-390; Directive 2003/86/CE du Conseil du 22 septembre 2003 relative au droit au regroupement familial, fOCE L 251 du 3.10.2003, p. 12-18; Directive 2003/109/ CE du Conseil du 25 novembre 2003 relative au statut des ressortissants de pays tiers résidents de longue durée, fOCE L 16 du 23.01 .2004 , p. 44-53.

9 Cette réticence trouve une autre déclinaison dans le paragraphe 4 de l'article 79 TFUE, qui précise : «Le Parlement européen et le Conseil, statuant conformément à la procédure législative ordinaire, peuvent établir des mesures pour encourager et appuyer l'action des États membres en vue de favoriser l'intégration des ressortissants de pays tiers en séjour régulier sur leur territoire, à l'exclusion de toute harmonisation des dispositions législatives et réglementaires des États membres ».

10 À titre non exhaustif : D. Acosta ArcazAro, «The Good, the Bad and the Ugly in EU Migration Law: Is the European Parliament Becoming Bad and Ugly? (The adoption of Directive 2008/15: The Returns Directive) », EfML, vol. 11., 2009, p.19-39 ; C. BERTRAND, « Les conditions d'une politique commune de l'immigration : apport et limites du traité de Lisbonne », Europe no 2, Février 2010, étude 2 ; R. CHOLEwINSKI, “The EU acquis on irregular migration ten years on: still reinforcing security at the expense of rights ?", in E. GUILD, P. MINDERHOUD (eds), The first decade of Eu migration and asylum law, Leiden, Martinus Nijhoff, 2012, 485 p., p. 127-177 ; P. DE BRUYCKER, « The European Border and Coast Guard : A new model built on an old logic », European Papers, Vol.1, 2016, n 2, p. 559-569; P. Devisscher, «Legal migration in the relationship between the European union and ACP countries : The absence of true global approach continues », EfML, vol. 13, 2011, p. 53-94; E. LopAtin, « The changing position of the European Parliament on Irregular Migration and Asylum under Co-decision », fCMS, 2013, vol. 51,
} 
Peu médiatisée, la dimension légale de l'immigration de ressortissants de pays tiers se situe au cœur de l'actualité législative. Elle constitue l'un des éléments clés sur lesquels la Commission Juncker a décidé d'axer son programme relatif à l'Espace de liberté, de sécurité et de justice. L'une des dix priorités poursuivies sur la période 2015-2019 est d'aller vers un « agenda européen en matière de migration $»^{11}$, sur la base du programme politique du candidat Juncker, qui ambitionnait avant son élection de développer une nouvelle politique en matière d'immigration légale ${ }^{12}$. Cet engagement se concrétise, moins d'un an après l'entrée en fonction de la nouvelle Commission, par l'adoption d'un agenda européen en matière de migration en mai 2015. Basé sur quatre piliers complémentaires, ce document doit fournir les lignes directrices nécessaires aux institutions de l'Union dans l'appréhension des problèmes posés par la migration. Prenant en compte l'asile, l'immigration illégale et le renforcement de la gestion des frontières extérieures, cette feuille de route fait de la migration légale son quatrième pilier. Elle prévoit qu' « un cadre clair et bien mis en œuvre relatif aux voies légales d'entrée dans l'Union (au moyen d'un régime d'asile et d'un système des visas efficaces) atténuera les facteurs d'incitation au séjour et à l'entrée dans des conditions irrégulières, contribuant ainsi à accroître la sécurité des frontières européennes et la sûreté des migrants $»^{13}$. Loin du simple rappel de principe, ce quatrième pilier envisage rien de moins que la mise en place d'un « nouveau modèle de migration légale $»^{14}$, après avoir réitéré le constat du manque d'efficacité de la politique actuelle.

La question de l'efficacité se situe au cœur des préoccupations de la nouvelle Commission. Comme le candidat du Parti populaire européen le prévoyait dans ses grandes orientations politiques, plusieurs instruments législatifs de la politique de migration légale vont faire l'objet d'examens détaillés peu de temps après sa prise de fonction. Jean-Claude Juncker envisageait par exemple de « réexaminer la législation sur la Carte bleue et sa mise en œuvre peu satisfaisante ${ }^{15}$. Moins d'un an plus tard, l'agenda européen sur la migration pointera lui aussi les difficultés rencontrées par ce dispositif ${ }^{16}$. Une consultation publique est prévue sur l'avenir de ce texte afin de pallier les difficultés relevées, qu'il convient d'appréhender en relation avec l'évaluation de l'acquis existant en matière de migration légale actuellement en cours. Mentionnée dans l'agenda sur les migrations et dans le programme REFIT adopté par la Commission en 2013, cette dernière a pour but « de répertorier les lacunes et les incohérences et d'étudier les moyens de simplifier et de rationaliser le cadre actuel de l'Union de manière à contribuer à une meilleure gestion des flux de migration légale ${ }^{17}$. La feuille de

$\mathrm{n}^{\circ}$ 4, p. 740-755 ; G. PAPAGIANNI, « Forging an external EU migration policy : from externalisation of border management to a comprehensive policy? », EfML, vol. 15., 2013, p. 283-299.

11 Voir le lien suivant qui résume les différentes priorités de la Commission européenne pour la période 2014-2019 : https://ec.europa.eu/ commission/priorities_fr

12 J.-C. JUNCKER, « Un nouvel élan pour l'Europe : Mon programme pour l'emploi, la croissance, l'équité et le changement démographique », Orientations politiques pour la prochaine Commission européenne, Discours d'ouverture de la session plénière du Parlement européen, Strasbourg, 15 juillet 2014, p. 10.

13 Commission européenne, Un agenda européen en matière de migration, COM (2015) 240 final, Bruxelles, 13 mai 2015 , p. 8.

14 Commission européenne, Un agenda européen en matière de migration, op. cit., p. 21.

15 J.-C. JUNCKER, «Un nouvel élan pour l'Europe : Mon programme pour l'emploi, la croissance, l'équité et le changement démographique », op. cit., p. 10.

16 Commission européenne, Un agenda européen en matière de migration, op. cit., p. 18. La Commission rappelle à cette occasion que, « au cours de ses deux premières années d'existence, seules 16000 cartes bleues ont été délivrées, dont 13000 par un seul et unique État membre ». 17 Ibid., note de bas de page 38 : «La Commission procèdera à un examen et à une évaluation (fitness check) de l'acquis existant en matière de migration légale dans le but d'en répertorier les lacunes et les incohérences et d'étudier les moyens de simplifier et de rationaliser le cadre actuel de l'UE, de manière à contribuer à une meilleure gestion des flux de migration légale ». Cette formule est reprise telle quelle dans le programme d'évaluation législative en matière de migration légale : Commission européenne, Fitness check legal migration, Consultation strategy, 
route de cette évaluation législative prévoit un achèvement en janvier 2018, mais précise néanmoins que son intensité pourra varier en fonction de la longévité des instruments législatifs : « la pertinence et la cohérence de toutes les directives sera évaluée, mais le champ et l'ampleur de l'analyse sur les autres aspects sera différente $»^{18}$.

L'agenda européen des migrations et l'évaluation de l'acquis législatif amorcée depuis septembre 2016 indiquent que les objectifs du « Mieux légiférer » sont pris en compte par la politique de migration légale de l'Union. Comment se manifeste la transposition de ces objectifs à cette politique dans les faits ? La volonté de renforcer efficacité et transparence s'accorde-t-elle avec les spécificités propres à l'objet visé ? Le " Mieux légiférer » peut-il être efficacement transposé à la politique de migration légale, malgré son caractère clivant et la marge de manœuvre conservée par les États membres ? Plutôt que de dresser un panorama global de cette politique et de ses instruments, nous nous concentrerons sur l'un de ses textes emblématiques, la directive 2009/50/CE dite « Carte bleue » établissant les conditions d'entrée et de séjour des ressortissants de pays tiers aux fins d'un emploi hautement qualifié ${ }^{19}$. Résumant presque à lui seul la difficulté d'appliquer le « Mieux légiférer » au domaine de la migration légale, cet instrument fait aujourd'hui l'objet d'une proposition de refonte législative de la Commission ${ }^{20}$, dont le Parlement européen vient de finir la première lecture ${ }^{21}$. La présidence maltaise du Conseil l'a, quant à elle, intégrée à ses priorités en matière de migration légale lors de sa prise de fonction ${ }^{22}$. L'examen de l'application du « Mieux légiférer » à ce texte pilier de la politique de migration légale révèle un décalage entre l'ambition des objectifs affichés (1) et les limites de la méthode retenue pour les réaliser (2).

\section{1) Le « Mieux légiférer » et la directive «Carte bleue»: l'ambition des objectifs}

L'application du programme « Mieux légiférer » à la directive «Carte bleue » doit renforcer la cohérence et l'efficacité d'un dispositif clé de la politique de migration légale. La transposition de principes cardinaux du REFIT vise autant à honorer l'engagement politique de la construction d'une nouvelle politique de migration légale (1.1) qu'à réagir aux critiques récurrentes adressées précisément à l'encontre de cette directive (1.2).

\footnotetext{
Version 1.2.2017, p. 1 : «Its aim is to evaluate the existing EU legislation on legal migration ; try to identify any possible gaps or inconsistencies ; and analyse if it contributes to an effective management of migratory flows », disponible à l'adresse suivante : https://ec.europa.eu/home-affairs/ sites/homeaffairs/files/what-we-do/policies/legal_migration/fitness_check_legal_migration_-_consultation_strategy_1.2.2017_en.pdf .

18 Commission européenne, Evaluation and fitness check roadmap, Legal migration, $1^{\mathrm{er}}$ septembre 2016, p. 6, « the relevance and cohernece of all directives will be assessed, but the scope and depth of the analysis on other aspects will differ » (nous traduisons), disponible à l'adresse suivante : http://ec.europa.eu/smart-regulation/roadmaps/docs/2016_home_199_fitnesscheck_legal_migration_en.pdf.

19 Directive 2009/50/CE du Conseil du 25 mai 2009 établissant les conditions d'entrée et de séjour des ressortissants de pays tiers aux fins d'un emploi hautement qualifié, FOCE L 155 du 18.6.2009, p. 17-29.

20 Commission européenne, Proposition de directive du Parlement et du Conseil établissant les conditions d'entrée et de séjour des ressortissants de pays tiers aux fins d'un emploi nécessitant des compétences élevées, COM (2016) 378 final, Strasbourg, 7 juin 2016.

21 Parlement européen, Commission LIBE, Projet de rapport sur la proposition de directive du Parlement européen et du Conseil établissant les conditions d'entrée et de séjour des ressortissants de pays tiers aux fins d'un emploi nécessitant des compétences élevées (COM (2016) 0378, C8-0213/2016 - 2016/0176 (COD), 26 janvier 2017.

22 Programme national de la présidence maltaise, janvier 2017, p. 23 : « Dans le même temps, les efforts se poursuivront dans le domaine de la migration légale, afin de faire en sorte que l'Europe dispose des conditions nécessaires pour attirer les travailleurs possédant les compétences dont elle a besoin. En gardant cet objectif à l'esprit, la Présidence fera avancer les négociations sur la révision de la directive Carte bleue ».
} 


\subsection{Contribuer à la construction d'une nouvelle politique de migration légale}

Lors du discours précédant son élection à la présidence de la Commission prononcé devant le Parlement européen le 15 juillet 2014, Jean-Claude Juncker rappelait qu'il souhaitait « promouvoir une nouvelle politique européenne en matière de migration légale », en vue de remédier aux "pénuries de qualifications spécifiques et à attirer des talents afin d'être mieux à même de relever les défis démographiques qui se posent à l'Europe $»^{23}$. Afin de concrétiser cet objectif politique, de nombreuses initiatives vont être prises par le nouveau président de la Commission. Ainsi, est créée une Direction générale « Migration, Affaires intérieures et citoyenneté », dirigée par le Commissaire grec Dimitris Avramopoulos. Cette nomination vise à honorer l'engagement du président de la Commission, qui entendait conduire la politique migratoire de la nouvelle Commission dans un « esprit de solidarité $~^{24}$ en donnant une visibilité accrue aux États situés en première ligne sur le front migratoire. L'impulsion nouvelle donnée à la politique migratoire de l'Union doit se concrétiser par l'amélioration de la qualité de la législation, portée par l'impératif « Mieux légiférer ». L'application de cette stratégie au droit dérivé de la politique de migration légale de l'Union doit contribuer à renforcer sa légitimité et à montrer que la Commission met en œuvre les engagements politiques pris avant la nomination du nouveau président.

La mise en œuvre de l'évaluation réglementaire de la politique de migration légale constitue un jalon important de la tentative de re-légitimation de la Commission européenne depuis la prise de fonction de Jean-Claude Juncker. L'objectif de cette évaluation est, en effet, de repérer les éventuelles lacunes ou incohérences de la politique pour améliorer l'efficacité et l'adéquation de la législation en cause avec les objectifs de transparence et de cohérence structurant l'initiative « Mieux légiférer ${ }^{25}$. Cette évaluation lancée sous le mandat de la Commission actuelle trouve son origine dans une décision de 2013. La communication REFIT indiquait alors qu'une évaluation réglementaire de ce type serait effectuée sur la politique de migration légale ${ }^{26}$. La Commission Juncker saisit l'occasion de montrer qu'une action est entreprise en vue de renforcer la cohérence globale de la politique de migration légale de l'Union. Elle procède à une réutilisation politique de décisions prises avant son entrée en fonction, pour servir un même but de renforcement de sa légitimité sur l'un de ses textes les plus critiqués. Elle fait, sur ce dossier, preuve d'une attitude particulièrement volontariste. Outre l'évaluation réglementaire actuellement en cours, elle proposera également une révision législative à la suite d'un rapport sur la mise en ouvre du texte produit au premier semestre 2014.

23 J.-C. JUnckeR, «Un nouvel élan pour l'Europe : Mon programme pour l'emploi, la croissance, l'équité et le changement démographique », op. cit, p. 10.

24 Sur l'émergence du concept en matière de migration et sa transformation récente en solidarité flexible opérée sous l'impulsion de plusieurs États membres, voir P. De BruYcker, E. Tsourdi, « The Bratislava Declaration on migration : European irresponsibility instead of solidarity », OMNIA Odysseus Blog, 27 septembre 2016.

25 Commission européenne, «Terms of reference for a study in support of a Fitness Check and compliance assessment of existing EU legal migration Directives », disponible à l'adresse suivante : http://ec.europa.eu/home-affairs/sites/homeaffairs/files/what-we-do/policies/ legal_migration/tor-fitnesscheckstudyv15-clean-annex_i-v_en.pdf, p. 3.

26 Commission européenne, Programme pour une réglementation affûtée et performante (REFIT) : résultats et prochaines étapes, COM (2013) 685 final, Bruxelles, 2 octobre 2013, p. 13 de l'annexe. 
L'entreprise de renforcement de la légitimité de la Commission Juncker sur la migration légale, qui insiste particulièrement sur la directive «Carte bleue », se base sur des initiatives déjà engagées avant son entrée en fonction. Sans procéder à une remise à plat complète de la législation en matière de migration légale, la Commission Juncker réussit à insuffler son rythme propre à cette légitimation de la politique de migration légale, en s'appropriant les initiatives arrêtées antérieurement comme l'évaluation réglementaire, qu'elle fait précéder d'une initiative législative sur une nouvelle directive «Carte bleue » au printemps 2016. L'objectif poursuivi est de montrer que la nouvelle Commission appréhende le problème dans ses multiples facettes, en vue d'atteindre deux buts distincts. Il s'agit, bien sûr, d'essayer de concrétiser l'engagement politique du président Juncker et de renforcer ainsi la légitimité de la Commission. Il s'agit également de réagir aux critiques récurrentes adressées à l'encontre de la Commission sur la « Carte bleue ».

\subsection{Répondre à la récurrence des critiques}

Instrument phare de la politique de migration légale, la directive «Carte bleue » entre dans le champ de l'évaluation législative (fitness check) lancée en septembre 2016 après proposition d'une directive modificative le 7 juin $2016^{27}$. La feuille de route de cette évaluation précise que les résultats à venir seront utilisés « comme base afin de déterminer quelles actions pourraient être requises afin d'améliorer la cohérence de la législation en matière de migration légale $»^{28}$. L'application du programme « Mieux légiférer » à la politique de migration légale vise un renforcement de ses différents instruments. Une évaluation « horizontale » doit à cette fin être mise en œuvre en vue de déterminer la compatibilité du droit dérivé avec les principes directeurs du programme REFIT que sont la pertinence, la cohérence, l'efficacité, l'efficience et la plus-value européenne de l'action entreprise ${ }^{29}$.

La directive « Carte bleue » a déjà fait l'objet de plusieurs rapports et analyses, pour un constat sévère. Lors de son adoption en 2009, la directive « Carte bleue » précisait à son article 21 que la Commission serait tenue de produire un rapport sur son application au Parlement européen et au Conseil, « tous les trois ans et pour la première fois le 19 juin 2014 au plus tard [...] et propose, le cas échéant, les modifications nécessaires $»^{30}$. Sur cette base, la Commission a produit un rapport d'évaluation le 22 mai $2014^{31}$, qui pointe les nombreuses difficultés rencontrées. D’après ses conclusions, le nombre extrêmement faible de cartes délivrées sur la période étudiée impose de relativiser l'idée d'un quelconque renforcement de l'attractivité de l'Union pour les ressortissants d'État tiers hautement

\footnotetext{
27 Commission européenne, Proposition de directive du Parlement et du Conseil établissant les conditions d'entrée et de séjour des ressortissants de pays tiers aux fins d'un emploi nécessitant des compétences élevées, op. cit.

28 Commission européenne, Evaluation and fitness check roadmap, Legal migration, op cit., p. 1 : « The results of the Fitness Check will be used as a basis to assess what actions (both legislative and non- legislative) might be required to improve the coherence of the legal migration legislation ».

29 Ouverture, transparence, efficacité, voir Commission européenne, Programme pour une réglementation affûtée et performante (REFIT) : résultats et prochaines étapes, op. cit.

$30 \quad$ Directive 2009/50, op. cit., article 21.

31 Commission européenne, Communication au Parlement et au Conseil sur la mise en œuvre de la directive 2009/50/CE du Conseil établissant les conditions d'entrée et de séjour des ressortissants de pays tiers aux fins d'un emploi hautement qualifié (« Carte bleue européenne »), COM (2014) 287 final, Bruxelles, 22 mai 2014.
} 
qualifiés porté par la directive. Cette difficulté tenant au volume numérique des cartes délivrées se conjugue avec le problème des autorités émettrices. L'immense majorité des cartes distribuées est, en fait, issue d'un seul État membre ${ }^{32}$. D'après la Commission, l'attractivité du système mis en place par la «Carte bleue » est minée par la possibilité laissée aux États de conserver leurs systèmes nationaux, sur la base de l'article 79 § TFUE $^{33}$. Adoptée avant la réforme de Lisbonne, la directive « n'a finalement fixé que des normes minimales et a laissé une marge de manœuvre aux États membres grâce à de nombreuses dispositions facultatives et renvois à la législation nationale $»^{34}$. La Commission éclaire les difficultés sérieuses rencontrées par la pièce maîtresse du dispositif de l'Union en matière d'immigration qualifiée, sans proposer de modification substantielle. Deux ans plus tard, des conclusions similaires sont dressées dans l'étude d'impact qui accompagne la proposition législative de nouvelle «Carte bleue $»^{35}$. Ces rapports pointent de manière récurrente la possibilité de perpétuer les systèmes nationaux d'admission, ce qui réduit considérablement l'efficacité globale de la directive et confirme les interrogations récurrentes des ONG et du monde académique sur cette incohérence majeure.

Ce problème sera particulièrement mis en lumière par la Commission dans sa proposition législative communiquée au Conseil en 2016, qui propose la suppression pure et simple des systèmes nationaux au sein du nouveau dispositif de «Carte bleue ». La tentative d'application du « Mieux légiférer » à la directive « Carte bleue », en ce qu'elle tente de répondre aux critiques récurrentes adressées à l'encontre de la directive « Carte bleue » et de la politique migratoire dans son ensemble, doit participer à la construction d'une nouvelle politique de migration légale défendue par la nouvelle Commission. En proposant des solutions aux incohérences des textes de droit dérivé existants, elle s'inscrit clairement dans le cadre de la stratégie « Mieux légiférer » tout en essayant de formuler des réponses satisfaisantes aux critiques récurrentes adressées à la directive «Carte bleue ». Toutefois, le nombre de plus en plus important de documents de la Commission relatifs à la Carte bleue confine à la profusion. Aux documents cités, qui représentent des centaines de pages dédiées à la «Carte bleue », s'ajoute depuis septembre 2016 l'évaluation de l'acquis législatif dans le domaine de la migration légale. Alors même que l'on se cantonne à l'étude d'un seul instrument législatif, on est frappé par l'abondance des rapports, de documents dans lesquels la Commission reconnaît ses torts. Quels enseignements sur le « Mieux légiférer » peut-on tirer de ce constat?

$32 \quad$ Ibid. p. 3 : «En 2013, le nombre de cartes bleues délivrées, à la hausse, s'est établi à 15 261, celles-ci ayant essentiellement été délivrées en Allemagne (14 197) au Luxembourg (306) et en France (304)».

33 Ibid., p. 4 : «l'existence de dispositifs nationaux destinés aux travailleurs hautement qualifiés pourrait avoir une incidence sur le nombre de cartes bleues européennes délivrées par certains États membres ».

$34 \quad$ Ibid., p. 11

35 Voir les 6 annexes accompagnant la proposition de nouvelle carte bleue, Commission européenne, Proposition de directive du Parlement et du Conseil établissant les conditions d'entrée et de séjour des ressortissants de pays tiers aux fins d'un emploi nécessitant des compétences élevées, op. cit. 


\section{2) Le « Mieux légiférer» et la directive «Carte bleue» : les insuffisances de la méthode}

L'application du programme « Mieux légiférer » à la directive «Carte bleue » interroge sur la possibilité d'atteindre les objectifs poursuivis par l'évaluation de l'acquis législatif, indifféremment de la manière dont elle est organisée (2.1) ou des outils privilégiés pour sa mise en œuvre (2.2).

\subsection{Les carences de l'organisation}

L'organisation de la mise en œuvre des préconisations du programme « Mieux légiférer » à la directive « Carte bleue » conduit à douter de la possibilité d'atteindre les objectifs poursuivis, tant les carences sont nombreuses.

La logique ayant présidé à l'adoption du calendrier d'application du « Mieux légiférer » à la directive «Carte bleue » est difficilement compréhensible. L'évaluation législative susmentionnée est le troisième acte de ce type pris en trois ans à l'égard de la directive «Carte bleue ». Comment conjuguer cet élément avec l'impératif d'efficacité revendiqué par le programme « Mieux légiférer »? Cette contradiction entre l'objectif affiché et l'organisation arrêtée pour mettre en œuvre l'évaluation de l'acquis législatif amène à relativiser la portée de son application à la migration légale. Selon la feuille de route, il est convenu que la « Carte bleue » « ne sera pas complètement examinée une nouvelle fois, mais l'examen et l'étude d'impact entrepris dans [le contexte de nouvelle proposition législative de la Commission] rentreront dans cette évaluation ${ }^{36}$. Cette manière de procéder ampute manifestement l'audit entrepris sur la politique de migration légale d'informations cruciales quant à son efficacité et à la pertinence de ses instruments. Les arguments avancés au soutien de cette stratégie emportent difficilement l'adhésion, si bien qu'il est permis de se demander si l'évaluation enclenchée ne vise pas, en laissant à l'écart de nombreux éléments, à renforcer en définitive le statu quo.

Cette limitation n'est pas le seul motif de circonspection suscitée par l'organisation de la mise en œuvre du « Mieux légiférer ». La feuille de route de l'évaluation de l'acquis législatif précise en effet que les instruments les plus récents de la politique étudiée seront exclus du champ de l'analyse ${ }^{37}$. La principale raison avancée, à savoir leur adoption récente, soutient difficilement l'analyse. Certains de ces outils, comme la directive sur les travailleurs saisonniers, ont en effet commencé à s'appliquer en 2014. Le souhait de la Commission d'économiser ses ressources et ses moyens se justifie tout à fait dans un souci d'efficacité de l'action publique. On rappellera simplement que, depuis 2014, la directive « Carte bleue » a fait l'objet de trois rapports d'évaluation en comptant le processus actuel, duquel elle n'est pas totalement exclue.

\footnotetext{
36 Commission européenne, Evaluation and fitness check roadmap, Legal migration, op cit., p.6.

37 Et en particulier : Directive 2014/36/UE du Parlement européen et du Conseil du 26 février 2014 établissant les conditions d'entrée et de séjour des ressortissants de pays tiers aux fins d'un emploi en tant que travailleur saisonnier, op. cit. ; Directive (UE) 2016/801 du Parlement européen et du Conseil du 11 mai 2016 relative aux conditions d'entrée et de séjour des ressortissants de pays tiers à des fins de recherche, d'études, de formation, de volontariat et de programmes d'échange d'élèves ou de projets éducatifs et de travail au pair, FOUE L 132 du 21.05.2016, p. 21-57.
} 
La logique qui sous-tend cette exclusion est difficile à saisir. Sous prétexte de transpositions trop récentes, la Commission recommande d'exclure de l'examen global de la politique de migration légale de l'Union nombre de ses instruments. L'examen de la cohérence transversale des différents instruments ne pourra être réalisé de manière optimale, certains en étant partiellement ou totalement exclus. Cette situation est d'autant plus regrettable que cet examen constituait sans doute la principale plus-value de l'évaluation actuellement mise en œuvre. Si on note, de plus, que les textes écartés de cette évaluation sont tous des instruments au sujet desquels la compétence de la Commission ne faisait pas débat, on peut s'interroger sur le but poursuivi. Est-il réellement de renforcer la législation existante?

Quoi qu'il en soit, l'organisation du processus d'évaluation actuelle de la politique de migration légale apparaît largement perfectible. Elle ne permet pas de répondre aux objectifs de renforcement de la cohérence entre la directive «Carte bleue » et le reste du droit dérivé de la migration légale, et ne favorise aucunement l'émergence de synergies entre des textes qui revêtent pourtant une importance bien réelle au sein de cette politique publique. Cette analyse n'est pas démentie par l'étude rapide des moyens utilisés pour réaliser l'évaluation de l'acquis législatif.

\subsection{Les lacunes des moyens utilisés}

L'autre élément qui conduit à douter de la faisabilité des objectifs poursuivis et révèle le décalage entre l'ambition de l'application du « Mieux légiférer » et la méthode pour la concrétiser est le choix des moyens utilisés à cette fin.

L'audit horizontal mis en œuvre dans le cadre de l'évaluation réglementaire de la politique de migration légale doit se faire en quatre phases. Il s'articule autour de questions posées aux principales parties intéressées lors de la troisième phase de l'analyse, après avoir procédé à une analyse contextuelle des problèmes posés ainsi qu'à des recherches documentaires en vue de construire les questions pertinentes. Les annexes de la stratégie « Mieux légiférer» fournissent des exemples de questions susceptibles d'être posées afin de mettre en lumière les contradictions ou oppositions existant entre les différents instruments législatifs. La Commission en tire ensuite les conséquences et propose les mesures qui s'imposent.

Cette méthode classique d'évaluation réglementaire répond à un impératif répété à plusieurs reprises par la Commission, notamment dans sa Communication de 2017 sur l'application du droit de l'Union ${ }^{38}$. On la retrouve dans d'autres évaluations réglementaires déjà achevées, dans des secteurs aussi divers que les directives oiseaux et habitats, les dispositions relatives au droit de la consommation ou encore au raffinage des produits du pétrole ${ }^{39}$. La méthode choisie est identique à

38 Commission européenne, Le droit de l'UE : une meilleure application pour de meilleurs résultats, COM 2017 C18/10, janvier 2017.

39 Commission européenne, Commission StaffWorking Document, Fitness Check of the EU Nature Legislation(Birds and Habitats Directives) Directive 2009/147/EC of the European Parliament and of the Council of 30 November 2009 on the conservation of wild birds and Council Directive 92/43/EEC of 21 May 1992 on the conservation of natural habitats and of wild fauna and flora, SWD (2016)472 final, 16 décembre 2016 ; Commisison européenne, Commisison Staff Working document, « Sectoral fitness check of the petrolaeum refining sector », SWD(2015) 284/2, 
d'autres évaluations législatives. On peut toutefois se demander si des aménagements auraient pu être envisagés en raison du caractère sensible de la matière. Outre le fait que le principal document d'appui ne peut pas vraiment être considéré comme affûté lui-même ${ }^{40}$, la méthode retenue pour l'évaluation actuelle de la «Carte bleue » ressemble en de nombreux points à celle retenue lors de l'étude d'impact sur la nouvelle «Carte bleue » de 2016. La question de la pertinence de l'évaluation de la réglementation sur la «Carte bleue », un an à peine après la production d'une étude d'impact portant sur sa nouvelle version, mérite d'être posée. Il est probable que les parties prenantes interrogées lors de l'évaluation réglementaire de la "Carte bleue ${ }^{41}$ soient identiques à celles interrogées un an auparavant, ce qui pose une nouvelle fois la question de la synergie et de l'efficacité.

Cette question se pose également si on considère la dimension horizontale de l'examen de la législation réalisé pour la politique de migration légale. On peut en effet douter que cette enquête horizontale permette un examen complet de la législation, dans la mesure où certaines questions ne seront délibérément pas posées sur la directive «Carte bleue », pour lesquelles on préférera procéder à un renvoi aux études réalisées précédemment. La principale difficulté de la méthode retenue est sa similarité quasi parfaite avec celle utilisée pour conduire l'étude d'impact de 2016. La lecture conjointe de la factsheet sur la refonte de la directive «Carte bleue » et la stratégie REFIT adoptée moins d'un an après sur toute la dimension légale révèle un nombre important de similarités ${ }^{42}$, qui peuvent être interprétées comme les signes d'une grande coordination en la matière. On a toutefois du mal à voir comment la méthode retenue peut simplifier le droit de l'immigration légale, en particulier l'admission des ressortissants de pays tiers à des fins de travail hautement qualifié. Il s'agit davantage d'une entreprise de compilation d'études distinctes concernant la «Carte bleue » qu'une véritable analyse globale au sein de laquelle elle serait versée. Une adaptation de la méthode aurait-elle dû être envisagée ? La plus value pour la « Carte bleue » apparaît assez relative. Les quelques dérogations à la méthode générale dont bénéficie la «Carte bleue » mettent à mal la synergie et la cohérence de l'ensemble de l'examen du droit de la migration légale. Cela est regrettable dans la mesure où la confrontation croisée de la directive «Carte bleue » aux textes législatifs plus récents, totalement exclus de l'évaluation réglementaire réalisée, aurait pu se révéler intéressante pour la poursuite des objectifs portés par la stratégie «Mieux légiférer ».

\footnotetext{
7 janvier 2016 ; Commission européenne, Evaluation and Fitness checl roadmap of Consumer law, http://ec.europa.eu/smart- regulation/ evaluation/index_en.htm

40 Commission européenne, « Terms of reference for a study in support of a Fitness Check and compliance assessment of existing EU legal migration Directives », op. cit., document supposé expliquer la marche à suivre et dont on ne peut pas réellement dire qu'il soit facilement compréhensible.

$41 \quad$ Ibid., p.5 «The key stakeholders to be consulted are government administrations, trade unions, business, educational institutions, international organisations and civil society, including migrants' organisations ».

42 Commission européenne, Factsheet, Révision de la directive Carte bleue européenne, 7 juin 2016.
} 
Le processus d'évaluation réglementaire de la politique migratoire actuellement en cours ne permet que d'émettre des suppositions sur le résultat à venir de l'application du programme « Mieux légiférer » à la directive "Carte bleue », texte emblématique de la difficulté de légiférer en matière de migration légale. Une fois le processus terminé, il faudra s'interroger sur le devenir des recommandations élaborées par la Commission. Il est permis de douter que ces dernières soient éloignées de celles formulées dans l'étude d'impact accompagnant la proposition de nouvelle " Carte bleue » de 2016. Dans tous les cas, on ne voit pas comment la mise en application du « Mieux légiférer » permettrait de dépasser les écueils qui se dressent face à cette politique de l'Union, cristallisés dans la directive «Carte bleue ». On a du mal à voir, au-delà du caractère cosmétique de la révision proposée par la Commission, comment les préconisations pourront vraiment être mises en œuvre à cause de la clause prévue à l'article $79 \S 5$.

La nature des rapports entretenus par la directive « Carte bleue » et le « Mieux légiférer » reste incertaine. Cette relation témoigne-t-elle d'un contournement ou d'un remplacement de la stratégie légistique revendiquée par la Commission ? La réforme actuelle de la « Carte bleue » s'est affranchie des contraintes procédurales du programme « Mieux légiférer », dans la mesure où elle a été proposée par la Commission avant le début de l'évaluation réglementaire de la politique de migration légale. Assiste-t-on à un processus d'intériorisation du « Mieux légiférer » par la Commission, qui mettrait ses préconisations en œuvre sans faire référence au programme du même nom ? Il convient de rester prudent, dans la mesure où l'évaluation réglementaire de la migration légale était programmée depuis 2013. L'application implicite des préconisations du « Mieux légiférer » à la « Carte bleue » doit-elle être envisagée comme un succès ou un échec de cette stratégie légistique ? D'un côté, l'adoption des documents prospectifs sur la «Carte bleue » a pu entraîner une mise à l'agenda plus rapide de l'évaluation de l'acquis législatif. De l'autre, cette accélération peut être vue comme la volonté de s'affranchir des contraintes imposées par la future feuille de route mise en œuvre pour le fitness check. On peut supposer que la Commission prend ce programme tellement à cœur qu'elle l'intériorise et applique automatiquement ses préconisations. Une autre lecture laisse à penser que l'évaluation réglementaire sert d'autres fins que l'amélioration de la qualité de la législation. L'exemple de la «Carte bleue » le montre particulièrement bien. Les dates choisies laissent à peine le temps de lire les études d'impact que d'autres sont déjà commandées. Quel intérêt, si ce n'est celui de fournir de nouveaux contrats aux sociétés de consultance auxquelles ces opérations sont facturées ${ }^{43}$ ? Quelle efficacité pour l'action publique ? L'impact de l'évaluation entreprise promet en tout état de cause d'être extrêmement faible pour la directive « Carte bleue».

En définitive, on peine à déterminer le véritable but de l'application du programme « Mieux légiférer » au texte le plus emblématique de la politique de migration légale. L'objectif affiché d'amélioration de la législation est loin d'emporter l'adhésion, et il semble difficile de réussir à se projeter au-delà de l'affichage. On peut considérer que l'application du « Mieux légiférer » à la directive

43 Commission européenne, «Terms of reference for a study in support of a Fitness Check and compliance assessment of existing EU legal migration Directives », op. cit. Ce document dont le but est d'éclairer sur la stratégie pratique à mettre en œuvre pour procéder à l'évaluation législative de la politique de migration légale fait à 36 reprises référence au terme « contractor ». 
«Carte bleue » ne parvient pas à dépasser le statu $q u{ }^{44}$. Les principaux défauts de la première version du texte n'ont pas disparu, alors qu'on se trouve pour le moment au stade de la proposition législative. La proposition actuelle de réforme de la Carte bleue se rapproche d'une tentative de recyclage, et le « Mieux légiférer » apparaît finalement impuissant face à des forces qui dépassent la simple légistique.

Initiative visant principalement à la re-légitimation politique de la Commission, l'application $\mathrm{du}$ « Mieux légiférer » à la directive « Carte bleue » ne constitue pas autre chose qu'un instrument d'affichage politique, comme en atteste le décalage entre l'ambition affichée et l'organisation définie pour la réaliser. L'avenir dira si ces conclusions doivent être étendues à l'ensemble de la politique de migration légale de l’Union européenne. 



\title{
LE « MieuX LÉgIfÉreR » AU CHEVET de LA POLITIQUE COMME RCIALE COMMUNE DE L'UNION EUROPÉENNE Potentiels ET LIMITES D'UNE RECHERCHE DE LÉGITIMITÉ PAR LA PROCÉDURE
}

\author{
Gael LE Roux ${ }^{1}$
}

\section{Résumé}

Cet article examine de quelle manière le programme " Mieux légiférer » est susceptible de favoriser la poursuite des objectifs mis en avant par la Commission dans sa stratégie «Commerce pour tous » de 2015. Une analyse des initiatives récentes en matière de négociations commerciales permet de constater que les principes et instruments du programme sont abondamment utilisés par l'Union dans l'élaboration de sa politique mais que le recours à ceux-ci ne semble pas en mesure d'enrayer la crise de légitimité sans précédent que connaît l'une des plus anciennes politiques communes de l'Union.

\section{Abstract}

This article assesses how the "better regulation » program may contribute to reaching the objectives set by the European Commission in its 2015 "Trade for all strategy". An analysis of recent trade negotiation initiatives allows to observe that the principles and instruments promoted in the program are fully exploited by the European Union when conducting its trade policy. Yet those seem to have a limited impact as to answering the legitimacy crisis faced by one of the most ancient European common policies.

La politique commerciale (PCC) est l'une des plus anciennes politiques communes de l'Union². Elle est également devenue aujourd'hui l'une des plus contestées ${ }^{3}$. Cela peut globalement s'expliquer du fait des spécificités du processus décisionnel inhérent à la négociation internationale. C'est également une conséquence du vaste spectre d'enjeux que la PCC recouvre et donc des intérêts, souvent contradictoires,

\footnotetext{
1 Doctorant contractuel, Aix Marseille Univ, Université de Toulon, Univ Pau \& Pays Adour, CNRS, DICE, CERIC, Aix-en-Provence, France. Préambule du Traité de Rome 1957 : « Désireux de contribuer, grâce à une politique commerciale commune, à la suppression progressive des restrictions aux échanges internationaux ».

3 R. Hiault, « TTIP : la politique commerciale de l'Union sous le feu des critiques », Les Echos, 10 juillet 2016, https://www.lesechos. fr/10/07/2016/lesechos.fr/0211115315573_ttip---la-politique-commerciale-de-l-union-sous-le-feu-des-critiques.htm, consulté le 12 mai 2017.
} 
qu'elle met en jeu4. Il semble globalement que, àl'instar de bien d'autres politiques del'Union européenne, sa modernisation soit une condition sine qua non de sa pérennisation. La clé des réformes de la PCC se situe sans aucun doute dans sa légitimation ${ }^{5}$. Celle-ci implique la recherche d'un équilibre subtil entre l'efficacité de ses instruments ${ }^{6}$, complexifiée par le caractère polymorphe de la matière commerciale, et son appropriation par la société civile, qui perçoit les enjeux commerciaux comme étant de plus en plus directement importants pour les citoyens ${ }^{7}$. Dans sa nécessaire quête de légitimité la PCC est particulièrement tiraillée entre une volonté d'efficacité ('output' legitimacy) qu'elle souhaite faire perdurer et un besoin de représentativité ('input' legitimacy) qu'elle se doit de renforcer'.

Dans sa nouvelle stratégie commerciale publiée en octobre 2015, la commissaire Cecilia Malmström évoque certaines des pistes de modernisation de la PCC envisagées par la Commission. Tout d'abord, il est important de souligner que la Commission maintient inchangé son objectif fondamental d'efficacité, qui se traduit par l'accroissement des échanges internationaux pour soutenir la croissance économique ${ }^{10}$. Elle met néanmoins un accent particulier sur la dimension inclusive qu'elle entend donner à cette politique, comme l'illustre son intitulé «le commerce pour tous $»^{11}$. À cette fin, il est envisagé d'associer plus largement à l'élaboration et à la mise en œuvre des instruments de la PCC différents acteurs qui considèrent leurs intérêts comme étant insuffisamment pris en compte. Cette stratégie peut donc d'une manière générale être perçue comme une initiative visant à renforcer la légitimité de l'action de l'Union en donnant davantage de place à la participation et à la transparence. Enfin, un troisième élément qui ressort de cette stratégie est l'accent mis par la Commission sur les valeurs que l'Union entend défendre au travers de ses actions commerciales, et que la commissaire place au même niveau que la poursuite de ses intérêts ${ }^{12}$. Parmi ces valeurs figure « au premier rang » la promotion de « son modèle réglementaire $»^{13}$. Cette allusion fait écho à un autre vecteur de légitimité, qui trouve sa source dans la qualité des processus décisionnels de l'Union ('througput' legitimacy) ${ }^{14}$. Celui-ci repose principalement sur la probité de la procédure, la responsabilité de ceux qui y participent vis-à-vis des bénéficiaires, la transparence et l'ouverture ${ }^{15}$.

\footnotetext{
4 Plusieurs facteurs complexes, internes et externes à l'Union, peuvent être identifiés comme étant à l'origine de cette contestation. Ce travail n'entrera pas dans le détail de l'analyse de ceux-ci mais se contentera de mentionner la multiplicité des critiques concernant autant le processus de négociation et de conclusion de ces accords que le contenu des traités commerciaux « complets et approfondis » négociés depuis une dizaine d'années. 5 La notion de légitimité est utilisée dans cet article au sens «Wéberien », c'est-à-dire comme se traduisant par une acceptation générale par les sujets d'un régime politique, des actions et décisions de celui-ci, y compris celles qui vont à l'encontre de leurs intérêts particulier, M. Weber, Économie et société, Pocket, 2003, 424 p.

6 Sur les rapports entre efficacité et légitimité sur le plan du droit voir notamment : M. Fatin-Rouge Stefanini, L. Gay, A. Vidal-Naduet (éd.), L'efficacité de la norme juridique : nouveau vecteur de légitimité?, Bruylant, 2012, 352 p.

$7 \quad$ Sur l'inclusion de la société civile au processus décisionnel comme vecteur de légitimité, voir J. HABERMAs Droit et démocratie. Entre faits et normes, Gallimard, 1996, 560 p.

Sur les notions d' « input legitimacy » et d' « output legitimacy », voir: F.W. SHARPF, Governing in Europe, Oxford University Press, 1999. Commission européenne, Commerce pour tous : vers une politique de commerce et d'investissement plus responsable, octobre 2015.

10 Commission européenne, Commerce pour tous : vers une politique de commerce et d'investissement plus responsable, octobre 2015, partie I. 11 Pour un commentaire sur ce point voir notamment : S. GsTöHL, « Trade for all - all for trade ? : the EU's new strategy », College of Europe Policy Brief, $n^{\circ}$ 3.16, Janvier 2016.

12 Commission européenne, Commerce pour tous : vers une politique de commerce et d'investissement plus responsable, octobre 2015, propos introductif de Cécilia Malmström.

13 Commission européenne, Commerce pour tous : vers une politique de commerce et d'investissement plus responsable, octobre 2015, propos introductifs et p. 23

14 V. Schmidt « Democracy and legitimacy in the European Union revisited : input, output and 'throughput'” Political Studies. Vol. 61, 2013, p. 2. La Professeure Vivien SchmidT, qui a théorisé cette forme de légitimation par la procédure et son importance pour l'Union européenne, a souligné qu'elle permettait de concilier les éléments parfois contradictoires qui pouvaient exister entre la poursuite d'une légitimité acquise par l'efficacité et celle garantie par la mise en place de processus participatifs et délibératifs garantissant la représentativité.

15 V. Schmid, « Democracy and legitimacy in the European Union revisited: input, output and 'throughput'” Political Studies. Vol. 61, 2013 , p. 6.
} 
Dans l'accord interinstitutionnel du 13 avril 2016 $6^{16}$, le Parlement, le Conseil et la Commission rappellent leur attachement à ces trois vecteurs de légitimité : la légitimité démocratique de l'Union ${ }^{17}$ (input legitimacy), la méthode communautaire ${ }^{18}$ (throughput legitimacy), une législation simple, claire et cohérente et transparente ${ }^{19}$ (output legitimacy). Les initiatives récentes de la Commission en matière de « Mieux légiférer » apparaissent, quant à elles, comme le principal outil de légitimation de l'action de l'Union fondé sur la qualité de son processus décisionnel. Dans la communication « Mieux légiférer » adoptée par la Commission le 19 mai 2015 ${ }^{20}$, celle-ci s'engage à « ouvrir davantage (le) processus d'élaboration des politiques, ainsi que mieux écouter et mieux interagir avec ceux qui mettent la législation de l'UE en œuvre et qui en bénéficient $»^{21}$. La Commission reconnaît également qu'elle ne devrait pas « imposer les politiques, mais bien ouvrir leur processus d'élaboration, dans un esprit de transparence totale et de pleine coopération, en tenant compte de l'avis de ceux qui sont concernés par la législation, de sorte que celle-ci soit facile à mettre en œuvre $»^{22}$. Il s'agit bien là de gages de légitimité, que la Commission entend donner par le biais d'une plus grande ouverture des processus décisionnels, et qui renvoie directement aux principes mis en avant dans la théorie sur la légitimité procédurale évoquée ci-dessus.

Les initiatives promues par l'Union dans le contexte du « Mieux légiférer » semblent donc aisément transposables à la PCC qui paraît déjà évoluer dans cette direction en faisant sienne les objectifs évoqués dans ces différents documents. Plus spécifiquement, la légitimité procédurale, que le programme « Mieux légiférer » de la Commission promeut, paraît être une voie particulièrement intéressante pour la modernisation de la PCC.

Au-delà des objectifs qu'elles poursuivent et des principes sur lesquels elles sont fondées, les initiatives en matière de "Mieux légiférer » s'appuient également dans la pratique sur un certain nombre d'instruments dont la fonction principale est d'améliorer les procédures décisionnelles. Les plus importants d'entre eux sont les études d'impact, les consultations publiques et les évaluations ex post $^{23}$. Ce sont ces outils et leur usage dans le cadre de la PCC qui feront l'objet de cette étude. Plus précisément, nous analyserons dans quelle mesure ceux-ci permettent à l'Union de recouvrer la légitimité qu'elle recherche dans le domaine de la politique commerciale commune.

Dans cette optique, nous procéderons à une évaluation de la teneur, de l'usage et de la portée des outils du programme « Mieux légiférer » sollicités dans le cadre des négociations commerciales internationales. Nous opérerons cette analyse à la lumière des théories sur la légitimité évoquées en

\footnotetext{
16 Accord interinstitutionnel « Mieux légiférer» 13 avril 2016, I.2. FOUE, L 123/2. Cet accord entre le Parlement, la Commission et le Conseil de l'Union est une révision d'un accord du même type adopté en 2003 et dans lequel les trois institutions adoptent une ligne de conduite commune en matière de Mieux légiférer.

17 Accord interinstitutionnel « Mieux légiférer » 13 avril 2016, I.2. FOUE, L 123/2, article 2.

18 Accord interinstitutionnel « Mieux légiférer » 13 avril 2016, I.2. FOUE, L 123/2, article 2.

19 Accord interinstitutionnel « Mieux légiférer » 13 avril 2016, I.2. FOUE, L 123/2, considérant 2.

20 Commission européenne, Améliorer la réglementation pour obtenir de meilleurs résultats - enjeu prioritaire pour l'UE, COM (2015) 215

final, 19 mai 2015. L'adoption de cette communication marque la dernière étape majeure d'une politique en faveur de l'amélioration de la législation européenne, initiée au début des années 2000.

21 Ibid., p. 3.

$22 \quad$ Ibid. p. 4.

23 Accord interinstitutionnel « Mieux légiférer » 13 avril 2016, I.2. FOUE, L 123/2, considérant 6, et partie III
} 
introduction. Nous nous concentrerons dans cette étude uniquement sur l'usage de ces instruments dans le cadre du volet externe de la politique commerciale, c'est-à-dire les négociations de traités commerciaux conduites par la Commission en vertu des règles édictées à l'article 207 § et l'article 218 du Traité sur le fonctionnement de l'Union européenne ${ }^{24}$. C'est en effet dans la négociation des traités commerciaux que l'Union fait face aujourd'hui aux plus grandes difficultés. C'est donc sur ce point qu'il nous paraît le plus pertinent de nous concentrer.

Dans un premier temps, nous analyserons comment la Commission utilise en matière de politique commerciale tous les outils mis en avant dans le programme « Mieux légiférer » (1). Nous verrons ensuite comment l'Union fait également un usage original de ces outils dans le cadre de la PCC, en en faisant la promotion auprès de ses partenaires (2).

\section{1) Le «Mieux légiférer» dans les négociations commerciales de l'Union}

Bien que la politique commerciale ne soit pas citée comme une priorité de la Commission dans ses principales communications récentes sur le «Mieux légiférer $»^{25}$, celle-ci en utilise les outils lorsqu'elle ouvre, négocie et conclut des accords. D’une manière générale, on constate le basculement d'une politique fondée sur des considérations macroéconomiques, soutenues par des études d'impact et des évaluations (1.1), à une politique qui cherche à asseoir son action sur une meilleure prise en compte des intérêts des différentes parties prenantes (1.2).

\subsection{La prépondérance des études d'impact : garanties d'efficacité}

Sur son site internet, la Direction générale du commerce de la Commission met en avant les trois types d'outils d'évaluation de sa politique commerciale dont elle fait usage lors de négociations internationales : les études d'impact, les sustainability impact assessment (SIA), et les évaluations ex post $^{26}$. Ces études ont des objectifs proches mais interviennent à différentes phases du processus de négociation. Traditionnellement, les négociations commerciales s'ouvrent sur la base d'une ou plusieurs études d'impact réalisées au préalable (ex ante) et qui servent de base à l'élaboration des lignes directrices qui constituent le mandat confié par le Conseil à la Commission européenne. On retrouve cette pratique dans les initiatives récentes de la Commission, qui s'est appuyée sur des études économiques multiples pour justifier l'ouverture de nombreux fronts de négociations simultanées ${ }^{27}$. Des études conduites pendant les négociations, appelées « sustainability impact assessment » sont

\footnotetext{
24 Cela signifie que nous ne nous attarderons pas sur le volet interne de la PCC qui correspond à l'adoption d'actes législatifs en matière commerciale adoptés unilatéralement par l'Union, par exemple concernant les modalités de fonctionnement de ses instruments de défense commerciale.

25 Commission européenne, Améliorer la réglementation pour obtenir de meilleurs résultats - enjeu prioritaire pour l'UE, COM(2015) 215 final, 19 mai 2015; Améliorer les résultats pour une Union plus forte, COM (2016) 615 final, 14 septembre 2016.

26 Commission européenne - Direction générale du commerce, "Policy evaluation" http://ec.europa.eu/trade/policy/policy-making/analysis/ policy-evaluation, consulté le 15 mai 2017.

27 On peut citer à titre d'exemple, l'étude d'impact réalisée pour le compte de la Commission par le Centre for Economic Policy Research et qui a conduit à l'ouverture des négociations du TTIP : CEPR, "Reducing transatlantic barriers to trade and investment”, Final project report prepared under framework contract TRADE10/A2/A16, 2013, J. FRANçOIS (éd.).
} 
également commanditées par la Commission ${ }^{28}$. Elles ont pour but d'orienter et d'éclairer les choix des négociateurs européens au cours des négociations ${ }^{29}$. Enfin, les évaluations sont également conduites pour analyser les effets de ces accords, après leur conclusion et pendant leur mise en œuvre ${ }^{30}$. Le tout conduit à l'élaboration d'une politique commerciale assez classique, car fondée sur des perspectives de croissance économique générée par l'accroissement des échanges commerciaux et calculée sur la base de modèles économiques empruntant pour la plupart aux théories des écoles de pensée dominantes.

Ces études sont réalisées dans la pratique par des sous-traitants mandatés par la Direction générale du commerce de la Commission européenne. Récemment, et sans doute en partie sous l'influence du nouvel accord interinstitutionnel adopté en 2016 $6^{31}$, le Parlement a également diligenté ses propres études, ce qui n'est pas encore le cas du Conseil ${ }^{32}$. La marche vers une plus grande variété des commanditaires de ces travaux (et donc potentiellement des méthodologies qui les sous-tendent) qu'encourage le programme « Mieux légiférer » va dans le sens d'une meilleure analyse de l'impact réel de ces accords commerciaux. Il en est de même de la prise en compte accrue des facteurs sociaux et environnementaux, qui affine cette démarche d'évaluation sans toutefois modifier sa logique fondamentale. Les conclusions auxquelles arrivent ces études d'impact demeurent en effet, dans la pratique, assez homogènes et globalement favorables aux initiatives envisagées par la Commission. Cela conduit de plus en plus de voix à réclamer une plus grande diversité dans ces études et à repenser leur utilisation ${ }^{33}$.

Si ces études, comme elles le prétendent, permettent d'orienter en amont, et d'évaluer en aval les initiatives en matière commerciale, elles sont un outil précieux pour des négociateurs confrontés à des choix stratégiques complexes lors de discussions avec les partenaires de l'Union. Elles peuvent à ce titre constituer un vecteur important d'efficacité de la politique commerciale commune. Elles ne semblent plus, en revanche, bénéficier de beaucoup de crédibilité aux yeux du grand public, et ne suffisent pas à elles seules à procurer à la Commission la légitimité recherchée.

\footnotetext{
28 Toujours concernant le TTIP, on peut citer l'étude réalisée par le cabinet ECORYS «SIA in support of the negotiations on a Transatlantic Trade and Investment Partnership », 30 mars 2017.

29 Voir à ce sujet: Commission européenne - Direction générale du commerce, "Handbook for trade sustainability impact assessments" second edition, 2016

30 Ainsi, l'accord avec la Corée du Sud conclu en 2011 a fait l'objet d'un rapport d'évaluation initial (mais pas encore d'un rapport final) publié en 2016. Seules ont été finalisées à ce jour les évaluations de l'accord préférentiel UE-CARIFORUM en 2014, et UE-Chili en 2012. Détails sur : http://ec.europa.eu/trade/policy/policy-making/analysis/policy-evaluation/ex-post-evaluations/, consulté le 15 mai 2017.

31 Accord interinstitutionnel du 13 avril 2016, point 17 « il appartient à chacune des trois institutions de déterminer comment elle organise son travail d'analyse d'impact, y compris ses ressources organisationnelles internes et le contrôle de la qualité ».

32 Voir une analyse de l'étude d'impact du TTIP conduite pour le compte du Parlement européen par le CEPS, “The impact of TTP : the underlying economic model and comparison » J. PeLKMANs (éd.), TTIP series Vol.1, No. 93, octobre 2014.

33 Voir par exemple la Déclaration de Namur, présentée en 2016 par 40 universitaires (à l'initiative du Ministre-Président de la Wallonnie, Paul Magnette), dans laquelle est demandée la conduite "d'analyses contradictoires et publiques des effets potentiels d'un nouveau traité », p. 1 ; Voir également un document publié fin 2016 par le gouvernement de la République française: « propositions françaises de réforme de la politique commerciale européenne », dans lequel il est réclamé à la Commission la publication d'au moins «trois études socio-économiques, sur une base scientifique pluraliste en mobilisant des écoles de pensée économique clairement distinctes » p. 3.
} 


\subsection{La consultation des parties prenantes : gages de représentativité}

D'une politique commerciale très axée sur l'analyse prospective des résultats macro-économiques (fondée sur les différents types d'études précitées), la Commission passe peu à peu à une politique davantage orientée vers la prise en compte d'intérêts différenciés. Ceci témoigne d'une certaine prise de conscience s'agissant des dynamiques complexes de redistribution des gains et coûts économiques engendrés par la conclusion de ces accords ${ }^{34}$. Cette transition reflète également de manière beaucoup plus évidente, le changement d'orientation stratégique vers une valorisation d'un processus décisionnel plus inclusif, soutenu par la Commission dans sa communication «Commerce pour tous » de 2015. En matière d'accords commerciaux, on retrouve ainsi un usage classique de la consultation des parties prenantes avant l'ouverture des accords ${ }^{35}$. On relèvera également l'institutionnalisation d'un cadre de consultation propre à la négociation commerciale, les civil society dialogues, dont l'objet est de permettre un échange régulier entre les fonctionnaires de la Direction générale du commerce et les représentants d'intérêts de la société civile (entreprises, ONGs, organisations publiques) sur divers sujets concernant la politique commerciale, parmi lesquels les négociations en $\operatorname{cours}^{36}$. On notera finalement la fusion progressive entre cet instrument et les études d'impact, puisque ces dernières s'appuient de plus en plus souvent sur des consultations qui font parfois partie intégrante de leur méthodologie ${ }^{37}$.

Malgré ces initiatives et les efforts sans précédents déployés par la Commission en matière de transparence dans le cadre des négociations du TTIP, on ne peut que constater l'insuffisance pour une partie de la société civile, de la multiplication de ces procédés de consultation comme vecteurs de légitimation. Ceci est sans doute dû à l'usage historiquement inégal des différents instruments du «Mieux légiférer » dans les phases successives du processus de conclusion d'accords commerciaux qui vont de la préparation du mandat à la mise en œuvre, en passant par la négociation. Les études d'impact semblaient globalement privilégiées pour les étapes les plus importantes, alors que les consultations paraissaient plutôt confirmer des orientations stratégiques déjà actées. Les variations d'usage de ces outils ont néanmoins elles-mêmes évolué pour des raisons tenant aux nouvelles orientations du programme « Mieux légiférer » et de la stratégie commerciale de l'Union. Si le tournant en faveur d'une plus grande ouverture aux parties prenantes renforce la légitimation de l'action de l'Union, celle-ci semble toujours insuffisante tant l'impact réel des consultations sur les procédures de négociation et de conclusion des accords est difficilement perçu par la plupart des citoyens.

Des pistes d'amélioration peuvent néanmoins être mentionnées. Le développement du recours aux études d'impact par d'autres acteurs que la Commission (Parlement européen, Conseil, États

\footnotetext{
34 Cette évolution progressive de la prise en compte des effets contrastés de la conclusion de ces accords est confirmée par la publication récente par la Commission européenne d'un « Document de réflexion sur la maîtrise de la mondialisation », 10 mai 2017.

35 À titre d'exemple, cinq nouvelles consultations publiques ont été lancées depuis le début de l'année 2017 : http://trade.ec.europa.eu/ consultations/

36 Voir sur le site de la Direction générale du commerce de la Commission européenne la page consacrée au Civil society dialogues : http:// trade.ec.europa.eu/civilsoc/

37 Commission européenne - Direction Générale du Commerce "Handbook for trade sustainability impact assessment" second edition, 2016, p. 10.
} 
membres, parlements nationaux) constitue peut-être une solution pour renforcer la légitimation de l'action de l'Union, à travers l'association d'une expertise sinon plus objective, du moins diverse dans son origine. D'une certaine manière, la fusion entre la consultation et l'étude d'impact consolide aussi la légitimation par l'amélioration des procédures. Ces outils méritent d'être testés, car ils présentent une alternative intéressante entre un basculement total vers une politique commerciale excessivement politisée qui risquerait de perdre complètement en efficacité et une politique commerciale exclusivement fondée sur l'expertise, dont la légitimité serait indéniablement contestée.

\section{2) Le «Mieux légiférer » comme vecteur d'affirmation de l'identité de l'Union}

Une des originalités de la politique commerciale est qu'elle participe à la projection du modèle réglementaire de l'Union au plan international ${ }^{38}$, aussi bien en matière de contenu des normes qu'en matière de pratiques législatives et réglementaires.

Sur le site internet dédié à l'amélioration de la législation européenne, la Commission évoque explicitement le rôle des accords commerciaux comme pendant externe de son initiative interne en matière de "Mieux légiférer $»^{39}$. Dans la pratique, cette logique l'a notamment conduite à intégrer au sein de ses accords commerciaux les principes et objectifs du « Mieux légiférer » dans les chapitres portant sur la « coopération réglementaire ». C'est notamment le cas dans le CETA et le TTIP, premiers traités commerciaux de l'Union à prévoir un chapitre détaillé de ce type ${ }^{40}$. La logique qui sous-tend cette démarche est double. D'une part, elle doit permettre de renforcer l'efficacité de ces traités dont l'objectif principal est de surmonter les barrières au commerce liées aux contraintes réglementaires issues des divergences de normes entre l'Union et ses partenaires ${ }^{41}$. D'autre part, ce type de chapitre doit renforcer la légitimité de l'Union dont la qualité du système normatif est présentée comme un modèle à suivre, au-delà de ses frontières ${ }^{42}$.

Concrètement, nous pouvons distinguer deux volets dans la démarche de la Commission dans les deux accords précités. Un premier concerne le rapprochement du contenu des normes entre les partenaires commerciaux, et fait écho à la gestion du stock réglementaire interne de l'Union qui répond aux exigences de réduction des coûts induits par la législation (2.1). Un second concerne les bonnes pratiques législatives et réglementaires, et s'adresse au flux législatif traité par les institutions en faisant la promotion d'un processus décisionnel de qualité, calqué sur celui de l'Union européenne (2.2).

\footnotetext{
38 A. R. Young, "The European Union as a global regulator: context and comparison” fournal of European Public Policy, Vol 22, n 9, juin 2015.

39 Commission européenne, «Améliorer la réglementation : pourquoi et comment? » https://ec.europa.eu/info/law/law-making-process/ better-regulation-why-and-how_fr\#objectives

40 Des cadres de coopération réglementaire plus ou moins sophistiqués ont précédé ces accords, notamment créés sous l'impulsion de l'OCDE, (voir notamment « Recommandations du Conseil concernant la politique et la gouvernance règlementaires » (2012), p. 20) mais leur intégration dans un traité commercial est apparue pour la première fois avec le CETA : Pour un compte rendu général de cette évolution, voir : Bernard Hoekman - « Fostering transatlantic regulatory cooperation and gradual multilateralization » fournal of International Economic Law, Vol. 18 n$^{\circ} 3,2015$, pp. 609-624.

41 Voir à ce sujet le discours de Cecilia Malmström, « Trade in the 21st century : the challenge of regulatory convergence », 19 mars 2015 http://trade.ec.europa.eu/doclib/docs/2015/march/tradoc_153260.pdf.

42 I. MANNNERs, « Normative power Europe: a contradiction in terms? » fournal of Common Market Studies, Vol 40, n 2. p. 235-58.
} 


\subsection{Rapprochement du contenu des normes : la gestion du stock}

La communication « Mieux légiférer » de 2015 souligne la nécessité d'une « gestion dynamique » de la législation de l'UE ${ }^{43}$. Pour ce faire, elle associe à ses initiatives sur l'amélioration de la législation, le programme REFIT qui a été lancé en $2012^{44}$. L'accord interinstitutionnel reprend également à son compte le programme REFIT présenté comme moyen « d'actualiser et de simplifier la législation » ${ }^{45}$. L'objectif central du programme REFIT est la réduction « des coûts de la législation » liés à une réglementation inadaptée ${ }^{46}$. Il est reflété dans certaines dispositions des chapitres sur la coopération réglementaire, inclus dans les accords de libre-échange les plus récents de l'Union européenne, et dont l'objectif général est aussi de réduire les coûts de la législation existante ${ }^{47}$.

Les similitudes relatives à l'objectif commun de réduction des coûts se traduisent par l'inclusion de mécanismes de mise en ouvre de nature très proche. Tout d'abord, les « bilans de qualité » prévus dans la communication de 2012, qui permettent de «dresser l'inventaire des domaines de la réglementation et des textes législatifs pour lesquels le potentiel de simplification des règles et de réduction des coûts réglementaires pour les entreprises et les citoyens est le plus important $»^{48}$, sont assimilables aux examens de la législation existante prévus dans le CETA $^{49}$ ou dans le projet de TTIP ${ }^{50}$. Ces accords prévoient en effet des moyens de passer au crible les législations internes de chaque partenaire afin d'identifier celles qui sont susceptibles de faire l'objet d'une convergence par le biais de «méthodes approfondies de coopération telles que la recherche de reconnaissance mutuelle, voire l'harmonisation $»^{51}$. Une autre similarité réside dans l'existence de la «plateforme REFIT », créée au moment de l'adoption du paquet « Mieux légiférer » de $2015^{52}$. Elle est quant à elle assimilable au « programme conjoint annuel en matière de coopération réglementaire entre l'Union européenne et les États-Unis ${ }^{53}$ dont l'objectif est de permettre une gestion transparente et inclusive des initiatives en matière législative et réglementaire. Dans la pratique, il s'agit dans les deux cas d'un site internet dans lequel sont recensés les textes existants et les initiatives en cours de préparation afin que celles-ci puissent être analysées et commentées par les parties prenantes concernées ${ }^{54}$.

43 Commission européenne, Améliorer la réglementation pour obtenir de meilleurs résultats - enjeu prioritaire pour l’UE, COM (2015) 215 final, 19 mai 2015, p. 11.

44 Commission européenne, Pour une réglementation de l'Union européenne bien affûtée, COM (2012), 746 final, 12 décembre 2012.

45 Accord interinstitutionnel « Mieux légiférer » 13 avril 2016, I.2. fOUE, L 123/2, point 48.

46 Commission européenne, Pour une réglementation de l'Union européenne bien affûtée, COM (2012), 746 final, 12 décembre 2012 , p. 2.

47 On retrouve explicitement cette idée de réduction des coûts dans les dispositions suivantes de chapitres sur la coopération réglementaire: CETA, article 21.3 (b) vi ; et (c) ii, (d) i ; TTIP, projet d'article X1 (1) d.

48 Commission européenne, REFIT: pour une réglementation de l'UE bien affûtée, 12 décembre 2012 p. 4.

49 CETA, article 21.4 (o) : « conducting post-implementation reviews of regulations or policies »; CETA article 21.4 (r) : "identifying the appropriate approach to reduce adverse effects of existing regulatory differences on bilateral trade and investment in sectors identified by a party »

50 Draft TTIP chapter on good regulatory practices, article 7, 21 mars 2016 : « Each party shall offer the opportunity for any natural or legal person to submit view to the relevant regulatory authority on improvements to existing regulatory frameworks »

51 L. GuIllenteguY, « les structures institutionnelles de coopération réglementaire dans les nouveaux accords commerciaux de l'UE », Présentation lors du colloque "Partenariats transpacifique et transatlantique à l'ère de l'interconnexion », UQUAM-Université de Rennes, Université d'Ottawa, 16-17-18 novembre 2016.

52 Commission européenne, Plateforme REFIT < https:/ec.europa.eu/info/law/law-making-process/overview-law-making-process/ evaluating-and-improving-existing-laws/reducing-burdens-and-simplifying-law/refit-platform/role-structure-and-working-methods-refitplatform_fr> consulté le 18 mai 2017.

53 TTIP, «EU proposal for chapter : regulatory cooperation » X.6 §2.

54 TTIP, «EU proposal for chapter : regulatory cooperation » X.6 §3; Commission européenne, Platrforme REFIT: <http://ec.europa.eu/ info/law/better-regulation/lighten-load_fr >, consulté le 18 mai 2017 
Enfin, le «forum de coopération réglementaire » instauré par le CETA, ${ }^{55}$ et le mécanisme institutionnel du même type (initialement dénommé « organe/conseil de coopération réglementaire » dans les premières propositions de la Commission sur lesquelles elle est depuis revenue) envisagé dans le TTIP ${ }^{56}$ sont des structures dont la composition et le rôle transversal et consultatif s'apparentent fortement à celui du « comité d'examen de la réglementation » instauré au moment de l'adoption du paquet « Mieux légiférer » de $2015^{57}$.

Les outils de cette gestion dynamique du stock législatif portés par l'Union sur la scène globale présentent une différence importante avec ceux utilisés au niveau interne liée à la différence de nature des coûts visés par ces mécanismes. Alors qu'au niveau interne, il s'agira de ceux qui sont induits par l'existence de dispositions législatives ou réglementaires inadaptées ou obsolètes, il s'agira au niveau international de ceux qui pèsent sur les échanges de biens, de services et d'investissements du fait des divergences substantielles de réglementation entre l'Union et ses partenaires commerciaux ${ }^{58}$. Cette différence entraîne une crainte liée à la remise en question de normes valides et équilibrées sur le simple fondement de leur incompatibilité avec une norme étrangère. De nombreuses critiques liées à cette crainte ont été exprimées dans le contexte des négociations du TTIP, dont les ambitions annoncées en matière de coopération réglementaire étaient les plus poussées, notamment sur le plan de leur institutionnalisation. La Commission a insisté sur le fait que ces mécanismes ne pourront pas remettre en question la poursuite par les parties d'objectifs internes de politiques publiques et le choix les moyens les plus appropriés pour y parvenir ${ }^{59}$. Malgré ces garanties affichées par la Commission, les critiques quant à la légitimité de ces mécanismes restent vives. Ils pourraient, selon certains auteurs, sinon court-circuiter, du moins sensiblement affecter les pouvoirs décisionnels des garants institutionnels de la représentativité démocratique ${ }^{60}$. Pour d'autres commentateurs, les contraintes que les parties acceptent volontairement de s'imposer dans le cadre de la coopération réglementaire, sont largement compensées par les gains en matière d'efficacité de la réglementation et de la législation adoptée dans un contexte global ${ }^{61}$.

La coopération réglementaire, lorsqu'elle concerne la gestion du stock législatif, voit donc s'opposer presque frontalement les partisans d'une légitimité fondée principalement sur la représentativité et ceux pour qui la légitimité repose sur l'efficacité. C'est donc dans une logique de conciliation que l'Union a mis l'accent dans le TTIP sur la recherche d'une plus grande légitimité procédurale, en se penchant principalement pour cela sur des principes de gestion homogène des flux législatifs.

$55 \quad$ CETA, article $21.6 \S 1$

56 TTIP, Annexe de la proposition révisée de chapitre sur la coopération réglementaire, 21 mars 2016.

57 Commission européenne, Comité d'examen de la réglementation : mission, tâches et composition, COM (2015), 3262 final, 19 mai 2015.

58 La commissaire Malmström les qualifie dans un discours prononcé le 19 mars 2015 de « unnecessary differences between different countries' regulations » (Trade in the 21st Century : the challenge of regulatory convergence) ; Dans le CETA l'article 21, 2 §4 mentionne: "unnecessary barriers to trade and investment".

59 Draft TTIP, «Proposal for a chapter on regulatory cooperation », préambule: “... does not reduce, undermine or otherwise compromise the level of protection in the relevant public policy areas", article 1, (b) "to contribute to the parties' activities pursuing public policy objectives .... Whilst facilitating trade and investment"; Annex - institutional set up for implementation: "All provisions of the regulatory cooperation chapter will be applied in full respect of the right to regulate to achieve public policy objectives".

60 S. LESTER et I. BARBEe, "The challenge of cooperation: Regulatory trade barriers in the Transatlantic Trade and Investment Partnership", fournal of International Economic Law, Vol. 16, $\mathrm{n}^{\circ}$ 4, p. 847-867, 2013, p. 865

61 J. B. Wiener et A. Alemanno, "The future of international regulatory cooperation: TTIP as a learning process toward a global policy laboratory", Law and Contemporary Problems, Vol. 78. n 4, p. 101-134, 2015 


\subsection{La promotion des bonnes pratiques législatives : la gestion du flux}

C'est davantage sur le terrain du flux législatif, constitué des actes en cours d'adoption ou non encore formellement proposés, que l'Union va pouvoir exporter son modèle réglementaire en se basant sur une légitimité tenant à la qualité de son modèle décisionnel et de ses procédures réglementaires. De la même manière que nous avions pu déceler des similarités entre le programme REFIT et les mesures destinées à modifier le stock réglementaire de l'Union et de ses partenaires commerciaux, nous pouvons faire plusieurs parallèles entre les principes encadrant les pratiques législatives et réglementaires prévues dans la communication de 2015 et repris dans l'accord interinstitutionnel de 2016, et ceux intégrés dans les chapitres (ou projet de chapitres) concernant les «bonnes pratiques réglementaires » du TTIP et du CETA ${ }^{62}$. Le titre II de l'accord interinstitutionnel d'avril 2016 sur la «programmation annuelle et pluriannuelle » (articles 4 à 11) fait ainsi écho aux mécanismes d'échange d'information précoces prévus dans le CETA $^{63}$ et dans le projet de chapitre « good regulatory practice» du TTIP proposé par la Commission ${ }^{64}$. Les dispositions sur les procédures de consultation de l'accord interinstitutionnel ${ }^{65}$ se reflètent quant à elles dans celles du CETA $^{66}$ et du TTIP ${ }^{67}$ qui prévoient l'ouverture du processus décisionnel aux parties prenantes. Les éléments du même accord interinstitutionnel concernant les études d'impact ${ }^{68}$ correspondent en substance à ce qui est intégré dans le projet de chapitre du TTIP ${ }^{69}$ et dans le CETA $^{70}$ s'agissant de l'évaluation des effets des mesures sur les acteurs à qui elles sont destinées. La Commission vise donc à faire des éléments de son programme « Mieux légiférer » des standards internationaux en matière de bonne pratique normative. Par-là, elle légitime son action interne en la présentant comme un modèle et influence également les procédures externes dans un sens qui lui est globalement favorable puisqu'elle favorise la convergence des normes dans le fond par le biais d'un rapprochement de leur mode d'élaboration.

Une réserve peut être apportée à cette démarche de transposition de ce type d'outil du programme « Mieux légiférer », du plan interne vers le plan externe. En effet, la critique générale adressée au chapitre sur la coopération réglementaire concerne leur manque de légitimité fondée sur la représentativité démocratique. En réponse à ce déficit structurel, des mesures procédurales

\footnotetext{
62 Dans le TTIP, cette distinction entre « coopération réglementaire » et " bonnes pratiques réglementaires » est clairement matérialisée (du moins dans les dernières propositions de mars 2016). Dans le CETA en revanche, ces deux aspects sont mélangés au sein d'un même chapitre (chapitre 21) dont l'objectif est mentionné à l'article 21, 4 §c « promote transparent, efficient and effective regulatory processes that support public policy objectives and fulfil the mandates of regulatory bodies, including through the promotion of information exchange and enhanced use of best practices".

63 CETA article 21. 4. (b) ; ( - i et ii) ; article 21.6 ; et surtout article 21.7 «the parties shall periodically exchange information of ongoing or planned regulatory projects in their areas of responsability »

64 TTIP, "EU proposal for chapter: good regulatory practices", article 3 'Internal coordination': "each party shall maintain internal coordination processes or mechanisms in order to foster good regulatory practices, including transparent planning, stakeholder consultation, impact assessments and retrospective evaluations of regulatory acts". Article $5 \S 1$ : "each party shall make publicly available at least once a year a list of planned major regulatory acts. Such list shall provide information on the scope and objectives of the regulatory acts"

65 Accord interinstitutionnel « Mieux légiférer » 13 avril 2016, I.2. FOUE, L 123/2, partie III, article 19

66 CETA, article 21.8 « ... each Party or the Parties may consult, as appropriate, with stakeholders and interested parties ... »

67 TTIP, "EU proposal for chapter: good regulatory practices" article 6 (§1 à §4): "stakeholder consultation" et article 7 "feedback on the existing regulatory framework".

68 Accord interinstitutionnel « Mieux légiférer » 13 avril 2016, I.2. FOUE, L 123/2, titre III articles 12 à 18

69 TTIP, "EU proposal for chapter: good regulatory practices", article 8

70 CETA, « Chapter 21: Regulatory cooperation », article 21.4, a) § 4
} 
visant à augmenter la transparence des processus législatifs et réglementaires et à associer aux processus décisionnels des représentants plus variés des différents intérêts qui seraient impactés par ces accords ont été proposées. Ce type de réponse procédurale, apportée à une question de légitimité démocratique, particulièrement prégnante dans le contexte de la politique commerciale, semble avoir un impact limité. Son degré devra être particulièrement avancé s'il veut pouvoir au moins partiellement compenser les problèmes de déficit de représentativité inhérents aux mécanismes de coopération réglementaire ${ }^{71}$.

La question de la légitimité de l'action de l'Union dans le domaine de la politique commerciale appelle une réponse globale. Celle-ci devra à la fois s'appuyer sur des ressorts classiques en misant sur la poursuite de résultats et une amélioration de la participation et de la représentation des citoyens, et à la fois innovante en se fondant sur des éléments de légitimation par une modernisation des procédures. Ce dernier vecteur de légitimité trouve des outils intéressants dans le contexte du programme « Mieux légiférer », utilisé de façon soutenue par l’Union en matière commerciale.

À l'heure actuelle, force est de constater que la légitimité de la PCC est toujours ébranlée en pratique. Des doutes peuvent donc être exprimés quant à l'efficacité de l'approche de légitimation par la procédure pour la renforcer. L'origine de ces doutes est déjà présente dans la théorie générale de la légitimité procédurale elle-même. En effet, il y est souligné un paradoxe inhérent à celle-ci : le respect et la qualité des procédures ne renforcent pas chez les citoyens le sentiment de légitimité, même si à l'inverse leur violation ou leurs défauts ont pour conséquence de faire disparaitre cette légitimité quand elle existait ${ }^{72}$. La légitimité procédurale que les initiatives en matière de «Mieux légiférer » peut apporter à la PCC n'est donc pas la panacée.

La recherche de l'équilibre entre une politique commerciale, dans laquelle les citoyens se sentent suffisamment représentés, et où cette représentation ne se traduit pas par une perte trop importante d'efficacité, reste donc à trouver. Un acteur dont le rôle en la matière était jusqu'ici plutôt secondaire pourrait acquérir une influence considérable dans ce contexte. Il s'agit de la Cour de justice de l'Union européenne. Celle-ci a en effet récemment pris deux décisions importantes concernant la politique commerciale commune, qui vont dans le sens de certaines demandes de la société civile en faveur d'une plus grande légitimation de l'action normative de l'Union dans ce domaine. La première concerne l'initiative citoyenne « stop TTIP » ${ }^{73}$, la seconde l'accord commercial UE-Singapour ${ }^{74}$. Dans la première affaire, le Tribunal sanctionne la Commission qui avait refusé d'examiner une initiative citoyenne visant à proposer au Conseil de revenir sur son mandat de négociation octroyé à la Commission au motif que cette demande sortait du cadre fixé par les règles

71 On peut par exemple imaginer faire participer des représentants du Parlement européen et/ou des Parlement nationaux au sein des forum de coopération réglementaire pour favoriser cette représentativité.

72 V. Schmidt « Democracy and legitimacy in the European Union revisited: input, output and 'throughput” Political Studies. Vol.61, 2013 , p.3. Trib. UE, 10 mai 2017, Michael Efler c./ Commission, T-754/14.

74 CJUE, Avis 2/15, 16 mai 2017. 
relatives à l'ICE. Dans la seconde, la Cour délimite le champ des compétences exclusives de l'Union dans le contexte des négociations d'accords commerciaux en excluant de celui-ci la protection des investissements. Ceci laisse entre les mains des États (et donc de leurs parlements nationaux), la charge de ratifier ce type de mécanisme dont la place dans les traités commerciaux internationaux est aujourd'hui incontournable. Dans les deux cas, les décisions du juge de l'Union octroient aux citoyens ou à leurs représentants un pouvoir plus important dans la conduite de la PCC, renforçant ainsi la légitimité fondée sur la représentativité au risque d'une certaine perte d'efficacité. 
TABLE DES MATIÈ RES

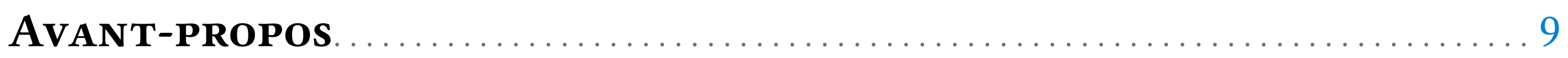

Nathalie RuBio

Préface.

Rostane MEHDI

Principaux Sigles et abréviations . . . . . . . . . . . . . . . . . . . . 13

Propos introductifs . . . . . . . . . . . . . . . . . . . . . . . . . . . . . . . . 15

Fabienne PÉRALdi LENEUF

1) L'évolution de la finalité technicienne de l'action «Mieux légiférer » .................................. 16

2) Les implications politiques de « Mieux légiférer » ................ 18

\section{Partie I}

COMPRENDRE LE «MIEUX LÉGIFÉRER

\section{Chapitre 1}

LE SENS DU «MIEUX LÉGIFÉRER»

BETTER REgULATION - VARIATIONS ON A THEME

Francis SNYDER

1) Origins.

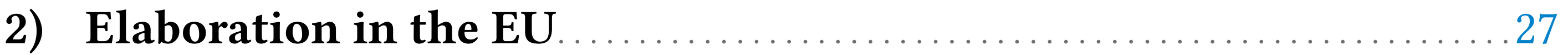

3) Competing Conceptions of Regulation ...........................28

4) Better regulation, the EU and International Economic Law .........31

LA CLARTÉ RÉ DACTIONNELLE EN PRATIQUE

Jennifer GRACIE LL.B.

1) Qu'est-ce que c'est la clarté rédactionnelle?

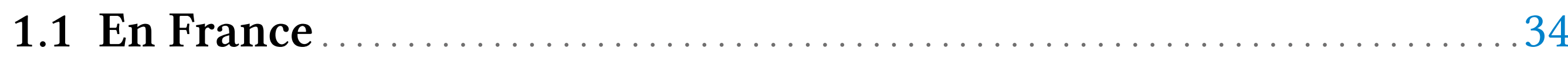

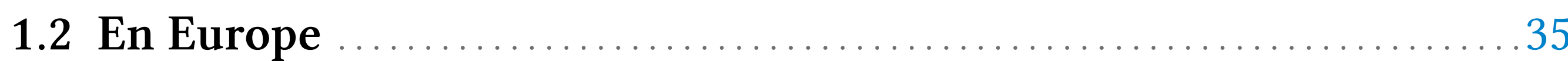


2) Comment rédiger de façon claire ?................................ 37

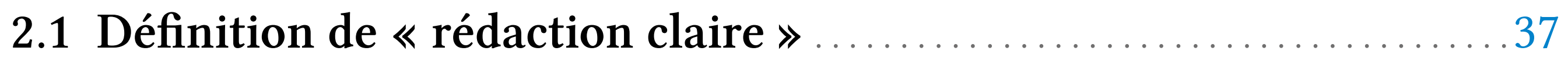

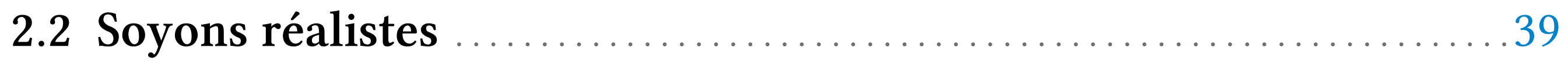

Soyons réalistes par rapport aux rédacteurs ........................... 39

Soyons réalistes aussi par rapport aux lecteurs.........................39

2.3 La solution pour la clarté rédactionnelle .......................... 39

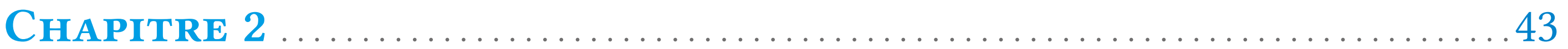

L'ORIGINALITÉ DU « MIEUX LÉGIFÉRER » AU REGARD DES EXPÉRIENCES INTERNATIONALES ET NATIONALES

Peut-on « Mieux Légiférer » en droit international ? . . . . . . 45

Romain LE BEuF

1) Les efforts internationaux en faveur du «Mieux légiférer »........ 47

1.1 Les tentatives de mieux légiférer par le droit international . . . . . . . 47

L'harmonisation du contenu des normes internes...................44 47

L'amélioration des procédures normatives internes....................49 49

1.2 Les tentatives de mieux légiférer en droit international . . . . . . . . . 50

Une aspiration constante........................................ 51

Des initiatives limitées ...................................... 52

2) Les obstacles internationaux au « Mieux légiférer » ...............54

2.1 Les obstacles tenant à la nature des sources du droit international . . 54

L'absence de procédure permettant de légiférer en droit international. . 54

L'impossibilité d'une réforme globale des modes

de production normative...................................... 56

2.2 Les obstacles tenant à l'ambiguïté axiologique

du droit international . . . . . . . . . . . . . . . . . . . . . . . . . . 58

L'hypothèse d'un «mieux» univoque................................58

Les risques d'une idéologie qui ne dit pas son nom ....................61 
LA CONCEPTION DU « MIEUX LÉGIFÉRER 》 EN DROIT CONSTITUTIONNEL . .63 Marthe Fatin-Rouge Stefanini

1) Les causes du particularisme français : des dispositions constitutionnelles rationnalisant le travail du Parlement

2) Les conséquences du particularisme français : le développement de normes constitutionnelles destinées à régir la production législative .. 66

2.1 La première période est celle allant de l'adoption de la Constitution de 1958 à 1998 et, précisément, à la décision du Conseil constitutionnel n 98-401 DC du 10 juin 1998

2.2 La deuxième période court de la décision du 10 juin 1998 jusqu'à la révision constitutionnelle du 23 juillet 2008

2.3 La troisième période s'ouvre avec la révision constitutionnelle du 23 juillet 2008

3) La faible efficacité du dispositif mis en place

\section{Partie II}

MetTRE en GEUVRE LE «MIEUX LÉGIFÉRER

Chapitre 1

Approches transversales de la mise en aUVRe DU « MIEUX LÉGIFÉ RER »

L'IMPACT DE LA CRÉATION DES ACTES DÉLÉGUÉS SUR LES MÉTHODES D'ÉLABORATION DU DROIT DE L'UNION EUROPÉENNE

LE CHEMIN VERS L'ENFER EST PAVÉ DE BONNES INTENTIONS

Laetitia Guilloud-Colliat

1) Le renforcement de la complexité dans l'élaboration du droit de l'Union, ou la difficile délimitation de l'acte délégué et de l'acte d'exécution

1.1 L'impossible distinction entre l'action de compléter et celle de préciser l'acte législatif.

1.2 Le débat sur la portée formelle ou substantielle d'une modification d'un acte législatif

2) La nécessité d'étendre aux actes délégués la lutte contre l'opacité de l'élaboration du droit de l'Union

2.1 Les critiques relatives à l'opacité de l'élaboration des actes délégués...89 2.2 Les efforts entrepris pour remédier à l'opacité des actes délégués ....91 
LE « MiEUX LÉGIFÉ RER 》 ET LA LiMitATION DU RECOURS AUX ACTES LÉGISLATIFS EN DROIT DE L'UNION

Stéphane DE LA Rosa

Les différents « temps » du programme « Mieux légiférer » ........996

La pluralité de sens du « Mieux légiférer » ....................... 98

Évolutions récentes du « Mieux légiférer »....................... 98

1) Le «Mieux légiférer » et la limitation du périmètre

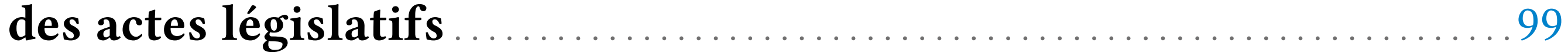

1.1 La limitation par la densification des études d'impact............ 100

1.2 La généralisation du retrait de propositions pendantes .......... 102

2) Le «Mieux légiférer » et la reconfiguration

des équilibres institutionnels

2.1 Le renforcement du contrôle de la Commission par la pratique du retrait .................................................. 104

2.2 La complexification de la procédure législative $\ldots \ldots \ldots \ldots \ldots \ldots$

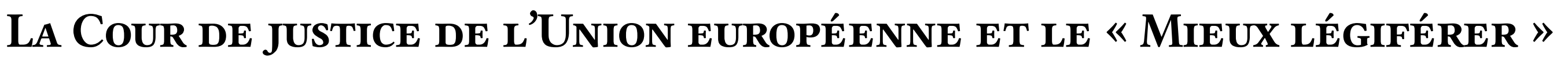
RECHE RCHE SUR L'IMPACT JURIDICTIONNEL

Nathalie RUBIo

1) Approche matérielle de la contribution de la CJUE au « Mieux légiférer»

1.1 Une référence exceptionnelle aux instruments du « Mieux légiférer»

1.2 Une jurisprudence en phase avec les exigences du « Mieux légiférer»

La garantie de l'ouverture et de la transparence

La garantie de la répartition des pouvoirs

2) Approche formelle et inter-institutionnelle de la contribution de la CJUE au «Mieux Légiférer »

2.1 La contribution par l'application et l'interprétation du droit de l'Union européenne

2.2 La contribution par la participation plus visible à l'élaboration du droit de l'Union européenne 
L'IMPACT DU « MIEUX LÉGIFÉRER 》 SUR L'INTÉGRATION DU DROIT DE L'UNION EUROPÉENNE DANS LES ÉTATS MEMBRES :

Le Mieux et Le Bien transposer

Emmanuelle SAULNIER-CASSIA

1) Une anticipation renforcée en amont de la procédure de transposition pour un Mieux transposer

1.1 Par une implication institutionnelle nationale coordonnée .. 133

1.2 Par l'analyse optimisée des impacts sur les droits nationaux ...135

2) Une «traçabilité » systématisée des opérations de transposition pour un Bien transposer

2.1 La clarification nécessaire des méthodes et des outils

de transposition

2.2 La cohérence des opérations de transposition

Chapitre 2

Approches SECTORIELles de LA Mise EN GEUVRe

DU « MIEUX LÉGIFÉRER »

«MieuX LÉgiférer »

L'EXEMPLE DES NOUVELLES TECHNOLOGIES DE L'INFORMATION Jean-Claude Bonichot

LES PRINCIPES DU « MIEUX LÉGIFÉRER 》 DANS LE DOMAINE DE L'ENVIRONNEMENT ET DE LA SANTÉ

QUel BILAN? QUelles PERSPECTIVES?

\section{Estelle BRossET}

1) La mise en application des principes du « Mieux légiférer »...... 161

1.1 Une importante mise en application .......................... 161

1.2 Une naturelle mise en application

2) La mise en tension des principes du «Mieux légiférer »

2.1 La mise en tension de la logique simplificatrice

2.2 La mise en tension de la logique expertale 
LA POLITIQUe Sociale À L'AUne du « MIEUX LÉgIFÉRER » 175

Dominique Nazet-Allouche

1) Le social à l'épreuve du «Mieux légiférer»

1.1 L'abandon des propositions précédemment émises . . . . . . . . . . . . 177

1.2 La multiplication des analyses d'impact ...................... 179

2) Les avancées sociales dans le respect du « Mieux légiférer »..... 181

2.1 L'élaboration d'un socle européen des droits sociaux

et les initiatives subséquentes

2.2 La rareté des nouvelles initiatives législatives

Éléments bibliographiques

AMbitions ET INSUffisANCES LÉGISTIQUES EN MATIÈRE

DE MIGRATION LÉGALE

L'APPLICATION DE LA STRATÉGIE « MIEUX LÉGIFÉRER 》

À LA DIRECTIVE «CARTE BLEUE »

Romain FOUCART

1) Le «Mieux légiférer » et la directive «Carte bleue » :

l'ambition des objectifs

1.1 Contribuer à la construction d'une nouvelle politique de migration légale

1.2 Répondre à la récurrence des critiques

2) Le « Mieux légiférer » et la directive «Carte bleue » :

les insuffisances de la méthode

2.1 Les carences de l'organisation

2.2 Les lacunes des moyens utilisés

LE « MieuX LÉgiférer » AU CHEVET de LA POLITIQUE COMMERCIALE COMMUNE DE L'UNION EUROPÉENNE

Potentiels ET LIMITES D'UNE RECHERCHE DE LÉGITIMITÉ

PAR LA PROCÉDURE

Gael Le Roux

1) Le « Mieux légiférer » dans les négociations commerciales de l'Union. 
1.1 La prépondérance des études d'impact : garanties d'efficacité ..... 204

1.2 La consultation des parties prenantes : gages de représentativité ...206

2) Le «Mieux légiférer » comme vecteur d'affirmation de l'identité de l'Union.

2.1 Rapprochement du contenu des normes : la gestion du stock ..... 208

2.2 La promotion des bonnes pratiques législatives : la gestion du flux ... 210

TABLE DES MATIÈRES 
UMR Droits International, Comparé et Européen (DICE)

Espace René Cassin

3 , avenue Robert Schuman

13628 Aix-en-Provence

dice-editions@univ-amu.fr

Composition et mise en page :

Donia Landoulsi, UMR DICE, Aix-Marseille Université

Conception de la couverture :

Donia Landoulsi, UMR DICE, Aix-Marseille Université

Illustration de la couverture :

Creative Commons CCO

$1^{\text {er }}$ trimestre 2018 
